\title{
Nonequilibrium Molecular Dynamics Simulation of the Thermocapillary Effect
}

\section{Molekulardynamische Nichtgleichgewichtssimulation des thermokapillaren Effekts}

Vom Fachbereich Maschinenbau an der Technischen Universität Darmstadt zur Erlangung des akademischen Grades eines Doktor-Ingenieurs (Dr.-Ing.) genehmigte DISSERTATION

vorgelegt von

Dipl.-Ing. Holger Andreas Maier

aus Alzenau

Berichterstatter: $\quad$ Prof. Dr.-Ing. Manfred J. Hampe

Mitberichterstatter: $\quad$ Prof. Dr. rer. nat. habil. Philippe A. Bopp

Tag der Einreichung: $\quad$ 27.06.2011

Tag der mündlichen Prüfung: 16.09.2011

Darmstadt 2011

D17 



\section{Summary}

Nonequilibrium Molecular Dynamics Simulation of the Thermocapillary Effect

Dipl.-Ing. Holger Andreas Maier

A natural convection occurs at lateral spatially variable solid-fluid or liquid-fluid interfaces [probstein1994]. It can play an important role in the transport of heat or mass across such interfaces, e.g. in evaporation or solvent extraction as they are often employed in chemical engineering [sherwood1975]. Model systems of such interfacial systems have been studied by different methods [colinet2001].

A very fundamental method is the usage of so-called molecular dynamics (MD) simulations [allen2003]. They model reality on a system of massive and charged particles (typically chosen so as to represent atoms), between which interparticle forces (chosen so as to represent intra- and intermolecular interactions) act, and solve the particle equations of motion under external constraints, that reproduce its interactions with the surroundings. Such a simulation could be considered as a numerical experiment, since the underlying assumptions are so fundamental that the system should, depending on their approximation of reality, also behave realistic on a larger scale in many situations.

We go beyond previous MD simulations of interfacial systems by considering also stationary nonequilibrium situations, which are technically more challenging to implement. An existing model system with liquid-liquid interfaces of Argonlike particles serves us as a starting point [buhn2004], [buhn2006], [bopp2008]. We implement a temperature gradient parallel to the interfaces by thermostating the particles in two opposite regions, so-called boundary regions, of the system to different temperatures.

Vortices localise close to the interfaces, with flow from hot to cold on each side along them. These vortices have all the characteristics ascribed to thermocapillary convection, the well-known Marangoni effect [weber1854], [thomson1855], [marangoni1871]. We shall thus assume its underlying effect (more generally known as the Marangoni effect) in action.

We then determine in detail the spatial distributions of different observables, that are related to the thermocapillary effect, and study their influences in parameter variations of the simulation system. Its flows depend, among other things, on the temperature gradient, the system geometry, and the viscosity of the liquids.

Finally, we compare the local behaviour at liquid-liquid with the one at other types of interfaces, e.g. liquid-gas and solid-fluid. Although the convection at them may differ at first sight, similar relations apply between the local observables as at liquid-liquid interfaces. Hence, we postulate a common underlying effect and make suggestions for further research into it. 


\section{Zusammenfassung}

Molekulardynamische Nichtgleichgewichts-Simulation des thermokapillaren Effekts

Dipl.-Ing. Holger Andreas Maier

An lateral örtlich veränderlichen fest-fluid und flüssig-fluid Phasengrenzen entstehen natürliche Konvektionen [probstein1994]. Sie können den Wärme- oder Stofftransport über die Grenze stark beeinflussen, z.B. bei in der Verfahrenstechnik weit verbreiteten Verdampfungs- oder Flüssig-Extraktionsprozessen [sherwood1975]. Modelle solcher Phasengrenzsysteme wurden bereits mit verschiedenen Methoden untersucht [colinet2001].

Die sogenannten molekulardynamischen (MD) Simulationen sind eine sehr fundamentale Methode [allen2003]. Sie bilden die Realität ab durch ein System aus masse- und ladungsbehafteten Teilchen (repräsentieren üblicherweise Atome), zwischen denen Kräfte (repräsentieren üblicherweise inter- und intramolekulare Wechselwirkungen) wirken, und lösen die Teilchenbewegungsgleichungen unter externen Randbedingungen, die seine Wechselwirkungen mit der Umgebung wiedergeben. Solch eine Simulation könnte man auch als numerisches Experiment bezeichnen, da ihre zugrundeliegenden Annahmen so fundamental sind, dass sich das System, je nach deren Realitätsnähe, auch auf größeren Längenskalen stets realistisch verhalten sollte.

Wir gehen in dieser Arbeit über frühere MD Simulationen von Phasengrenzsystemen hinaus, indem wir auch stationäre Nichtgleichgewichtszustände simulieren, die technisch aufwändiger zu implementieren sind. Ein bereits existierendes Modellsystem mit flüssig-flüssig Phasengrenzen aus Argon-ähnlichen Teilchen dient uns als Ausgangspunkt [buhn2004], [buhn2006], [bopp2008]. Wir implementieren einen Temperaturgradient entlang der Phasengrenzen durch Thermostatisieren der Teilchen in zwei gegenüberliegenden Bereichen, sog. "boundary regions", des Systems auf unterschiedlichen Temperaturen.

In der Nähe der Phasengrenzen bilden sich auf beiden Seiten Wirbel. Ihre Strömungsrichtung zeigt an der Grenze selbst von heiß nach kalt. Diese Wirbel haben alle charakteristischen Eigenschaften einer thermokapillaren Konvektion, wie sie der Marangoni-Effekt bewirkt [weber1854], [thomson1855], [marangoni1871]. Wir nehmen daher an, dass er im System auftritt.

Wir bestimmen anschließend im Detail die örtliche Verteilung verschiedener Observablen, die mit dem thermokapillaren Effekt in Verbindung stehen, und untersuchen deren Einflussfaktoren in Parameterstudien des Simulationssystems. Dessen Strömungen hängen unter anderem ab von dem Temperaturgradient, der Systemgeometrie und von der Viskosität der Flüssigkeiten.

Abschließend vergleichen wir das lokale Verhalten an flüssig-flüssig mit demjenigen an anderen Phasengrenzen, z.B. flüssig-gas oder fest-fluid. Obwohl die Konvektion an ihnen vordergründig anders aussehen kann, bestehen ähnliche Beziehungen zwischen den lokalen Observablen wie an den flüssig-flüssig Phasengrenzen. Wir postulieren daher einen gemeinsamen Effekt, der all diesen Strömungen und zugrunde liegt und machen Vorschläge zu dessen weiterer Untersuchung. 


\title{
References in the Summary
}

\section{Literaturverzeichnis zur Zusammenfassung}

\author{
Probstein R.F. 1994 \\ Physicochemical Hydrodynamics 2nd. ed., John Wiley \& \\ Sherwood T.K., Pigford R.L., Wilke C.R. 1975 \\ Colinet P., Legros J.C., Velarde M.G. 2001 \\ Allen M.P., Tildesley D.J. 2003 \\ Bopp P.A., Buhn J.B., Maier H.A., Hampe M.J. 2008 \\ Buhn J.B., Bopp P.A., Hampe M.J. 2004 \\ Buhn J.B., Bopp P.A., Hampe M.J. 2006 \\ Weber E.H. 1854 \\ Thomson J. 1855 \\ Sons, New York \\ Mass Transfer, McGraw-Hill, New York \\ Nonlinear Dynamics of Surface-Tension-Driven \\ Instabilities, Wiley-VCH, Berlin \\ Computer Simulation of Liquids, Oxford University Press, \\ Oxford \\ Chem. Eng. Communications 195 (11) 1437-1464 \\ Fluid Phase Equilibria 224 (2) 221-230 \\ J. Mol. Phys. 125 187-196 \\ Berichte über die Verhandlungen der königlich \\ sächsischen Gesellschaft der Wissenschaften; \\ Mathematisch-Physische Classe 1854 57-67 \\ Marangoni C. 1871 \\ The London and Edinburgh Philosophical Magazine and \\ Journal of Science 10 330-333 \\ Annalen der Physik und Chemie 143 337-354
}



About the author

1990-1999

Highschool

Friedrich-Dessauer-Gymnasium at Aschaffenburg, Germany

2000-2005

Student of Mechanical Engineering

Technical University at Darmstadt, Germany

2003-2004

Visiting Student

University of Illinois at Urbana-Champaign, USA

2005-2011

Scientific Assistant, Thermal Process Engineering Technical University at Darmstadt, Germany 



\section{Table of Contents}

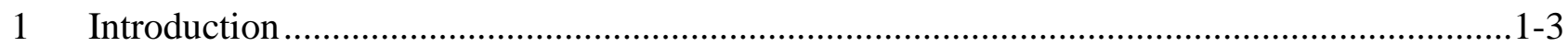

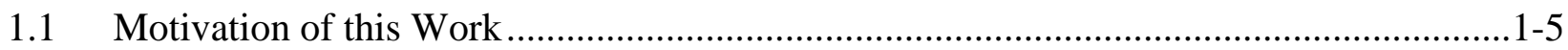

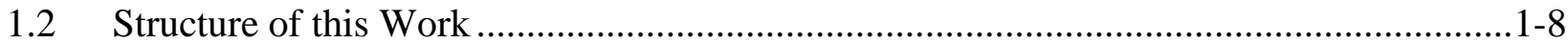

2 Review of the Interfacial Flows under a Temperature Gradient ...........................................2-10

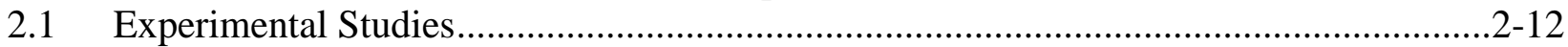

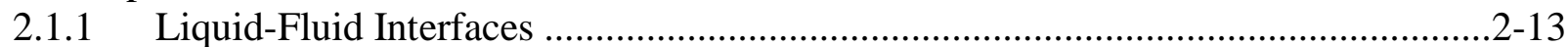

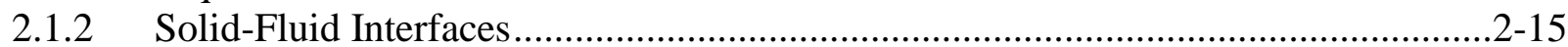

2.2 Heuristic Models of the Interfacial Phenomena ...........................................................2-18

2.2.1 Heuristic Boundary Conditions......................................................................2-22

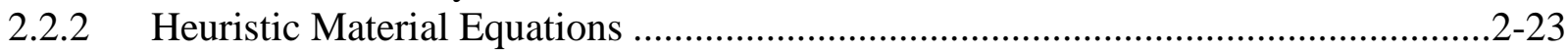

2.3 Fundamental Models for the Interfacial Phenomena ...............................................2-25

2.3.1 Maxwell's Explanation for the Thermal Creep ..................................................2-29

2.3.2 Advanced Models for the Interfacial Phenomena.....................................................2-32

2.4 MD simulations of the Thermal Creep at Solid-Fluid Interfaces ..................................2-34

3 Theoretical Background of MD Simulation......................................................................3-35

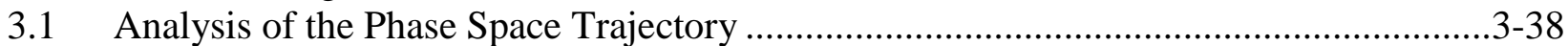

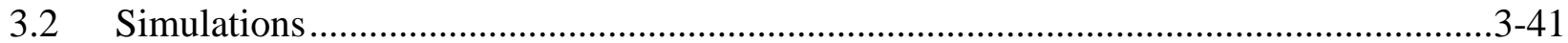

3.2.1 Equilibrium MD Simulations.......................................................................

3.2.2 Nonequilibrium MD Simulations....................................................................

$4 \quad$ General Strategy of this Work ………………………...............................................

4.1 Setup of the "Simulation Systems" ............................................................................ 48

4.1.1 General Setup of the Liquid-Liquid Interfacial Systems ..........................................4-52

4.1.2 Molecular Models and Force Fields........................................................................4-53

4.1.3 System Constraints...........................................................................................

4.1.4 Thermostating Algorithm................................................................................4-58

4.2 Expected Behaviour of our "Simulation Systems" ......................................................4-63

4.2.1 Equilibrium Interfacial Systems....................................................................4-65

4.2.2 Nonequilibrium One-Phase Systems .................................................................4-71

4.2.3 Nonequilibrium Interfacial Systems ...............................................................4-76

4.3 Analysis of our "Simulation Systems" ......................................................................... 4-80

4.3.1 Thermodynamic Observables ............................................................................

4.3.2 Hydrodynamic Observables ..............................................................................

4.3.3 Statistical Mechanical Observables .....................................................................4-88

4.3.4 Locations and Spatial Resolution of the Local Observables......................................4-89

4.3.5 Summarisation of the Local Data.......................................................................4-94

4.4 Preparational and Production Simulations ...................................................................4-100

4.4.1 First Equilibrium Interfacial System.............................................................4-102

4.4.2 First Nonequilibrium One-Phase Systems ........................................................4-105

4.4.3 First Nonequilibrium Interfacial System...............................................................4-107

4.4.4 Later Systems ...............................................................................................4-111

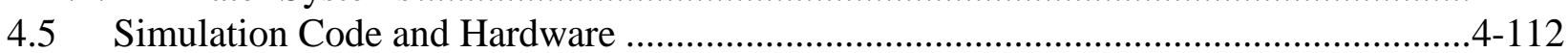

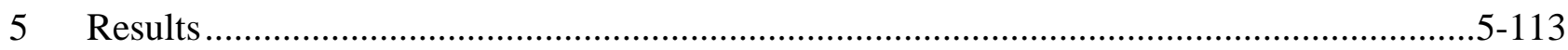

5.1 Approximation of Reality by our First Systems …………….................................

5.1.1 Equilibrium Interfacial System .....................................................................5-116

5.1.2 Nonequilibrium One-Phase Systems ………………………………………...5-122

5.1.3 Nonequilibrium Interfacial System....................................................................5-129 
5.2 Examination of the Parameters and Simplifications in the Heterophasic Systems ........5-138

5.2.1 Influence of the Cut-Off Radius on the Local Observables ...................................5-140

5.2.2 Influence of the Thermal Conductivity on the Thermostating Artefacts .................5-143

5.2.3 Influence of the Overmodulation by the Thermostats on the System Behaviour ...5-150

5.3 Homophasic Systems with a Better Approximation of Reality .................................5-153

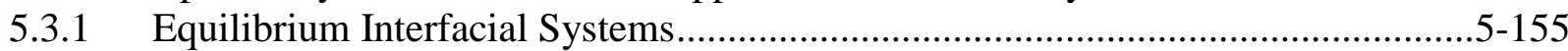

5.3.2 Nonequilibrium One-Phase Systems ……........................................................

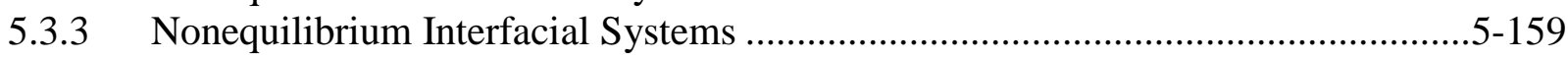

5.4 Examination of the Parameters and Simplifications in the Homophasic Systems .........5-162

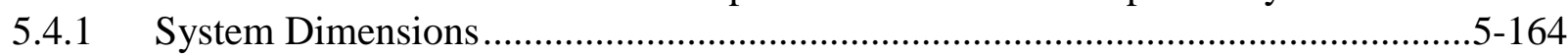

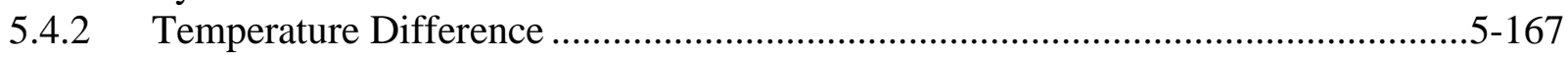

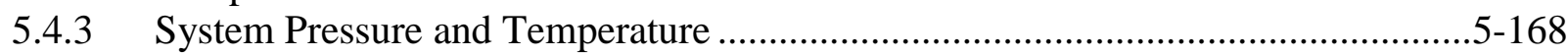

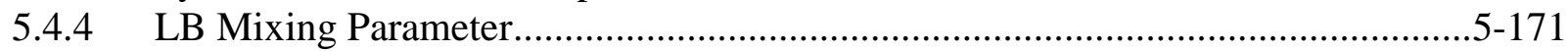

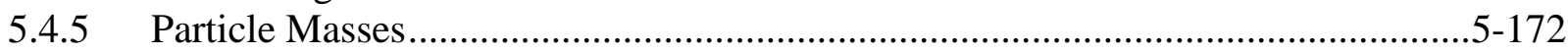

5.5 Advanced Studies of the Homophasic System Behaviour .........................................5-174

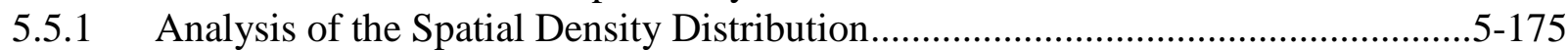

5.5.2 Analysis of the Local Stresses ..................................................................

5.6 Thermocapillary Convection in the Unary Inferfacial Systems .................................5-186

5.6.1 Liquid-Vapour Interfaces ......................................................................

5.6.2 Solid-Fluid Interfaces............................................................................

5.6.3 Liquid Phases Separated by an External Force ..................................................5-193

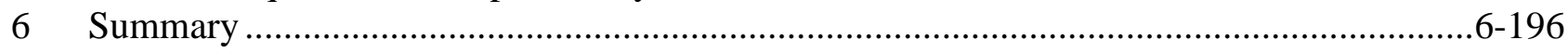

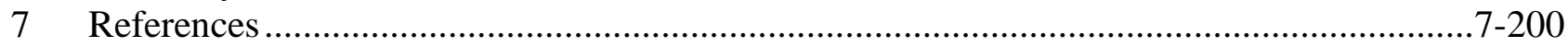

8 Appendix A: Fluctuations in Selected Observables...................................................... $8-216$

9 Appendix B: Basic Assumptions of Statistical Physics .....................................................9-218

10 Appendix C: Basic Assumptions of Irreversible Thermodynamics...................................10-220

11 Appendix D: Phase Behaviour of the Ar5Ar5 Mixture ................................................11-221

12 Appendix E: 2D Liquid-Liquid Nonequilibrium Interfacial System ..............................12-226

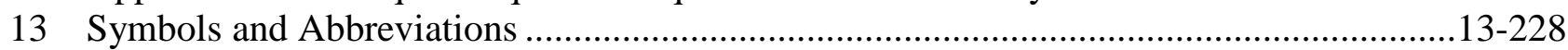

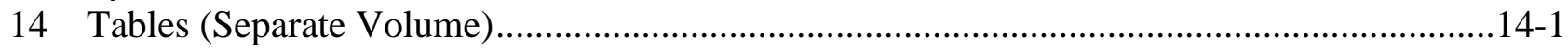

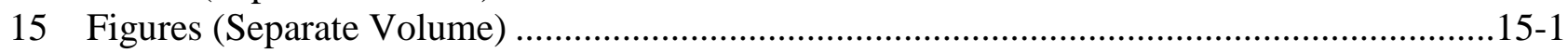




\section{Introduction}

Classical phenomenological equilibrium thermodynamics teaches that, depending on the external constraints, such as temperature, pressure, and composition, a mixture of components can exist either as a single homogeneous phase or as an ensemble of several phases. The number of phases as function of the composition and external conditions of the mixture are typically displayed in phase diagrams like the one shown in Figure 1. Such diagrams assume that the phases are in equilibrium, i.e. that the macroscopic quantities temperature, pressure, and chemical potentials are the same everywhere throughout the system.

In many real cases, however, the phases are not in equilibrium, as for example in systems in which two different phases were just brought into contact. Such a nonequilibrium system evolves only gradually towards an equilibrium state. Its establishment manifests itself through the occurrence of transport phenomena of mass, energy, and momentum within and between both phases. After the equilibrium state has been reached, both phases may have a composition strongly differing between them as well as from their respective initial states. Eventually, these phases can be separated again by mechanical means. Such "phase-contacting operations" are used in chemical engineering to achieve its objective: To separate mixtures of components. Accordingly, a profound understanding of both the equilibrium and nonequilibrium states is an important constituent for the design of chemical engineering processes. In particular the processes by which equilibrium is established starting from nonequilibrium states, which can span a wide range of time constants, should be investigated.

Macroscopically, the behaviour of equilibrium systems can be described, depending on the components more or less well, by conventional thermodynamics. Thus, the aforementioned Figure 1 compares, as an example, the experimental liquid-liquid equilibria of a binary water 1-butanol mixture at ambient pressure with ones modelled using the NRTL equation from the thermodynamics of mixtures [sorensen1979]. The parameter values underlying the model data in the figure were obtained by regression on the equilibrium measured at $25^{\circ} \mathrm{C}$. One can consider the coexisting phases in such a mixture, whatever their dimensions may be, as individual quasihomogeneous domains that are separated by two-dimensional interfaces. Mesoscopically, however, 
the situation is less clear. The interfaces are three-dimensional objects and should therefore better be called "interfacial regions".

The establishment of equilibrium from a nonequilibrium situation is much more difficult to describe than the equilibrium state. This is true at the macroscopic level, and even more so at the mesoscopic one. Any such description must necessarily proceed on a local and chronological basis in order to catch the heterogeneous and evolutionary character of the system. Classical phenomenological irreversible thermodynamics [haase1963], [degroot1960], a linear theory, has been able to describe the phase states as well as the transport processes inside and between the phases under certain circumstances. However, until today, there has been little progress in incorporating the interfaces themselves, where additional phenomena occur. These "interfacial phenomena" include, among others, the time-dependence of the interfacial structure and the interfacial rheology [cardin1991a], [stuke1961].

Another important class of interfacial phenomena, directly involved in the transport processes, are known as the Marangoni effects [weber1854], [thomson1855], [marangoni1871], [scriven1960], [sawistowski1971]. What makes understanding them particularly appealing is that they are known to greatly increase transport rates in and across interfaces over those from exclusively molecular transport mechanisms [colinet2001]. The Marangoni effects were recognised as the common cause of the convection observed experimentally in various interfacial systems. In all these experiments, the fluid at the interface is dragged along the latter in direction of decreasing interfacial tension. Such a spatially variable tension exists when the interface is subject to lateral gradients of temperature (thermocapillary effect), concentration (diffusocapillary effect), or electric charge (electrocapillary effect) [probstein1994]. The peculiarity of the thus resulting convection depends in each system on its specific external constraints. Note that the Marangoni convection should not be confused with buoyancy driven flows like the Rayleigh-Bénard convection [oertel2002], [ostrach1982].

Our investigations here focus on the thermocapillary effect. We shall study it particularly at liquidliquid interfaces, but others will be considered as well later on. The left side of Figure 2 shows, as an example, a laminar thermocapillary convection as observed in an experiment. See section 2.1.1 for further details. 


\subsection{Motivation of this Work}

One of the main reasons why interfacial phenomena in general are so little understood is the thinness of the interface. The "traditional approach" in physics to study such a phenomenon would be to interpret experiments on a definite system in terms of a phenomenological model relating the distortions and the responses [haile1997] of that system. This approach is illustrated at the top of Figure 3. After such a model has matured sufficiently, it can predict the system behaviour under different inputs and even be transferred to describe similar systems. In multiphase systems, the interfaces are often as narrow as a few nanometres and thus almost inaccessible to experiments. One then follows different approaches which can be subdivided into two groups:

The first group builds on the phenomenological models above, which manage to describe at least the behaviour of a separate phase. They are extended to apply also to the interfaces in multi-phase systems. Such extensions require additional more or less realistic assumptions on the properties and on the behaviour of the interfaces. Hence, one could call the resulting models "heuristic". The most popular ones take a continuum mechanics point of view of matter. However, all these heuristic models manage to reproduce only few selected phenomena at the interface and not its general behaviour [rowlinson2002].

The second group consists of more "fundamental approaches" which try to explain the behaviour of interfacial systems based on microscopic theories such as quantum-mechanics, or rather its classical limit. It models matter by a large number of mutually interacting particles which are subject to the laws of classical (Newtonian) mechanics. At any time, the microstate of such a model system can thus be uniquely specified by the positions and momenta of all particles.

It is Boltzmann's fundamental ansatz that averaging over a representative set of microstates in a system can produce any of its macroscopic observables [hill1986], [hill1987], [mcquarrie2000], [landau2007]. How these microstates are obtained is irrelevant as long as they comply with the macroscopic external constraints of the system, e.g. from thermodynamics or hydrodynamics. Figure 4 illustrates the fundamental approach in the classical limit graphically. Originally, 
Boltzmann's ansatz was developed for systems in thermodynamic equilibrium only. However, it is now applied as well to systems out of equilibrium.

Molecular simulations constitute the numerical realisation of Boltzmann's fundamental approaches. A representative set of microstates is computed by brute force. The recent advent of powerful computers has made molecular simulations very worth considering, as long as the phenomena occur on the length- and time scales accessible to these methods (typically a few nanometres and nanoseconds).

In particular Molecular Dynamics (MD) simulations, which solve the system's equations of motion, offer a wide spectrum of possibilities. So they allow not only for the computation of a set of microstates but also deliver them chronologically ordered in tuples, giving thus access to the evolution of the system on the microscopic level, see section $\mathbf{3}$ for details. Another advantage, that we want to leverage in this work, is that MD simulations can replace experiments, at least in principle [hockney1988], [frenkel2002], [haile1997].

Suppose, for example, that the velocity distribution in a Couette flow of a certain component under given boundary conditions is to be predicted. In hydrodynamics, a suitable model would require specific knowledge of several things such as the material properties, when the flow turns turbulent, whether energy dissipation must be accounted for, etc. . The input required for an MD simulation, i.e. essentially the interparticle interactions, is much more fundamental in nature. It is generally expected in science that a model at a more fundamental level can be simplified, or coarse grained, through suitable averaging procedures (or integrations) leading to more specific, and hopefully to more powerful, models for the specific properties to be investigated. Consequently, the hydrodynamic model should alternatively even be derivable from the results of MD simulation.

Due to their analogy with, and sometimes their ability to even replace the "physical experiment", MD simulations are also known as "numerical experiments". In some sense, the "numerical experiment" is even superior to the "physical" one since the measurements do not perturb the system, i.e. potentially bias the response. However, biases arise from other sources in application, see e.g. section 3 below. 
In this work, we shall apply MD simulations to circumvent the experimental inaccessibility of the interfacial region for our studies of the "thermocapillary effect". That is, we investigate the "thermocapillary effect" using simplified model reproductions of guiding liquid-liquid interfacial systems. The advantages of our "fundamental approach" are, of course, at the price of a more complex investigation procedure than it would be necessary in following the "traditional approach". We introduced it at the beginning of this section.

Before a simulation can be undertaken, the simulation system (i.e. the liquid, or liquids, or ...) and the stresses or constraints applied to it must be made to mimic the physical reality as closely as possible, keeping in mind the available material resources (i.e. computers, computer time etc.). Likewise, the response of the simulation system must, at the end, be transferred back to the real system. In the simulation itself, the investigation procedure remains, in principle, analogue to the experimental one. The simulation system is disturbed in a controlled manner and its response is measured while "measurement errors" are reduced as much as possible. They result from the statistical character of the simulation. Figure 3 illustrates this procedure again.

Modelling assumptions and technical simplifications contained in a simulation system itself or in its computational implementation, "measurement" errors, and the back-transfer to the real system will ultimately be the main causes that lead to a deviation between the responses of the simulation system and of the true system. To some degree, this deviation can be disentangled into its different causes by systematic variation of the assumptions and simplifications. It will be one of the major challenges of this work to identify in the system behaviour the trends against the uncertainties. 


\subsection{Structure of this Work}

Part 2 will review the general state of research into interfacial phenomena as far as relevant for the thermocapillary effect. We stated already in the previous section that we took a new approach by studying the thermocapillary effect in "numerical" instead of "physical experiments". This approach requires at first that their underlying systems, including the distortions, are modelled appropriately to be simulated. The "modelling assumptions" and "technical simplifications", that have to be made, ultimately bias the responses of the simulation systems. In part 3, we shall briefly introduce the basic assumptions and simplifications; they are standard and intrinsic to the MD simulation method. Additionally, its statistical physics background and general execution will be addressed.

Detailed information on the setup, simulation, and analysis of the various systems studied in the course of this work will be given in part 4. Although nonequilibrium interfacial systems, i.e. binary ones with liquid-liquid interfaces subject to a lateral temperature gradient, are the focal point of this work, we also studied corresponding equilibrium interfacial or nonequilibrium one-phase systems for reference. All nonequilibrium systems were simulated in so-called boundary driven (BD) MD simulations. In this method, the boundary regions of a system are perturbed specifically to establish, in our case, different target temperatures in them. Provided the system setup is suitably chosen, the perturbation removes the matter between the boundary regions into the desired nonequilibrium states. For better comparability most of the equilibrium systems were also simulated using this method. In doing so, all target temperatures within a system must merely be chosen identical.

All the aforementioned nonequilibrium and equilibrium systems were analysed in terms of few macroscopic observables: Namely ones that are defined as averages over the individual microstates of a system as for instance particle densities, temperatures, or centre of mass (com) velocities. Due to the special character of their definitions, we can determine these observables on-the-fly during our simulations to reduce their memory requirements and thus allow for relatively long runs. Nevertheless, the scales considerable still remain small in absolute terms so that the achievable reliability of the results is limited. Additionally, they involve further errors from various sources, such as the modelling assumptions, the technical simplifications, or numerical errors. In particular for the characteristic nonequilibrium system behaviour to emerge out of them, extreme temperature gradients of the order of $10 \mathrm{~K} / \mathrm{nm}$ are required. They make the occurrence of the thermocapillary 
effect, as known from experiments, appear questionable. Given these errors, we had to ascertain, prior to our actual studies, that the behaviour of our simulation systems displays the characteristic trends and features observed in reality. We checked this by comparing the local data against our expectations from experiments, theoretical considerations, or other MD simulations.

Part 5 will discuss the simulation results. As a matter of fact, already the behaviour of our first systems will turn out to match our expectations from chapter $\mathbf{4 . 2}$ quite well. There are only minor anomalies. Looking for a way to reduce them even more, we explored various manipulations to these first systems. Chapter $\mathbf{5 . 2}$ will discuss their responses. They concurrently tell us a lot about the influences on the thermocapillary convection. We used the thus obtained findings to reduce the simulation errors even more in later systems. Chapter $\mathbf{5 . 3}$ will compare their behaviour with the one of the first systems using selected representatives. We shall come to our actual studies of the thermocapillary effect in chapter 5.4. Individual parameters of our nonequilibrium interfacial systems were specifically varied so as to parallel the common alterations of a comparable system in an experimental study, such as for instance the system dimensions, of the temperature gradient. As intended in our "fundamental approach", the simulation results will show clearly how these alterations affect the system behaviour, and in particular the interfacial region, on the mesoscopic level. We shall address in chapter $\mathbf{5 . 5}$ certain aspects of the system behaviour again in more detail. Finally, chapter $\mathbf{5 . 6}$ will give a glimpse beyond the liquid-liquid interfaces at other ones, e.g. liquidgas or liquid-solid. They all have, different from one might expect at first sight, many local properties in common. We shall conclude this work in part $\mathbf{6}$ with a summary. 


\section{Review of the Interfacial Flows under a Temperature Gradient}

As already mentioned in part $\mathbf{1}$, an interface and the physical processes occurring there are usually not directly accessible to experiments due to its thinness. Those few interfacial properties that can be determined, such as the interfacial tension, hardly reveal anything about the microscopic processes involved. In favourable cases, an experimental signal, containing information about both the bulk phases (a lot) and the interfaces (a little), can be disentangled. It allows for some indirect conclusions on the influences of the interface and on their impact on the system. The existing phenomenological models of the thermocapillary effect were mainly derived from such approaches.

Countless studies of the interfacial flows in various setups have been reported in the literature. See, for instance, [levich1962], [nepomnyashchy2001], [velarde2001], [oertel2002], [naranayan2003], [probstein1994], [slattery2006]. We shall distinguish here, above all, the phenomena. As stated in the introduction, the thermocapillary effect itself is of particular interest to us. Hence, we will focus on interfaces which are subject to a lateral temperature gradient. Interfacial flows can occur as well for a different orientation but they then sustain, eventually, also a lateral temperature gradient on smaller scales (see section 2.1.1). We additionally focus specifically on the convection at liquidliquid interfaces. A realistic implementation of them in MD simulations requires only relatively few simplifying assumptions compared to other interfaces under a temperature gradient (see section 5.6). Nevertheless, our review includes also the convection at them. The findings there can help to extend our general understanding. We shall later use facts from this compilation to argue that our MD simulations reproduce the reality closely enough and to formulate the questions to be studied.

At first, we shall consider experimental studies of the interfacial flows (see chapter 2.1). Section 2.1.1 addresses the liquid-fluid, i.e. liquid-liquid or liquid-gas, interfaces where the so-called "thermocapillary convection" occurs. It looks totally different in a vast array of experiments. We shall rather compile its constituent features from them instead of going into the details. Section 2.1.2 will continue with the "thermal creep". It is the traditional name used for the convection at solid-fluid, i.e. solid-liquid and solid-gas, interfaces. We shall extract the actual influences and impacts of the creep from the three main experimental setups. In particular, the one of the Knudsen effect foretells, moreover, in some sense the behaviour of the liquid-liquid systems that we shall 
later study ourselves. Various phenomenological models of the interfacial flows will be introduced in chapter 2.2. They build mainly on hydrodynamics and thermodynamics. Section $\mathbf{2 . 3}$ will review analytical interfacial models deduced from more fundamental theories. In this context, the aforementioned solid-fluid interfaces are of particular interest again: Given a dilute gas, the thermal creep can be explained to a large extent at the microscopic level by invoking only the kinetic theory of gases. Such an explanation fails, however, for denser fluids. Since they can still exhibit thermal creep, we shall assume a more complex effect to be active in them. It is, presumably, a capillary one, as well. MD simulations in the literature show that the thermal creep in dense fluids always runs, similar to the thermocapillary convection, in the direction of decreasing interfacial tension. If this direction changes, e.g. when using a different wall potential, the creep flows invert as well. Chapter 2.4 will consider such fundamental simulation approaches to study the thermal creep. To our knowledge, they have not been made to the liquid-fluid interface yet. We want to catch up on them in this work. In fact, our simulation results in chapter $\mathbf{5 . 6}$ will confirm the postulated relationship between the thermocapillary convection and the thermal creep at higher fluid densities. Hence, we shall assume it, here, to be driven as well by a thermocapillary effect. 


\subsection{Experimental Studies}

There is a great similarity between the thermocapillary convection and the thermal creep. After all, they differ, basically, only in the direction of the interfacial flows with respect to that of the temperature gradient. We shall first look at the experimental findings at liquid-fluid interfaces (see section 2.1.1) and then at solid-fluid interfaces (2.1.2). 


\subsubsection{Liquid-Fluid Interfaces}

The thermocapillary convection can, depending on the system setup, occur in various ways in a liquid-fluid system. We describe them, for instance, for different orientations of the temperature gradient with respect to the interface below. Other factors include the composition and the thermodynamic state of a system, its dimensions, etc. . See also [schwabe1981] or [schatz2001] for more detailed reviews. The variety of the thermocapillary flows makes it hard to recognise at first sight the essential similarity that all experiments share: We shall describe it summarily as a drag of fluid in the vicinity of the interface, along the interface, in direction of decreasing interfacial tension. Since it decreases with the temperature in the vast majority of cases, the flows run typically from hot to cold. They invert, however, in the rare systems with an inverse temperature dependence of the interfacial tension. The larger its gradient is, the stronger are generally the flows, irrespective of their direction.

A dependence on the interfacial tension in the way described up to here characterises the thermocapillary convection. Buoyancy driven flows interfere with it in the earth's or in any other gravitational field. They result from the temperature dependence of the fluid density, in consequence of which the fluid is subject to a spatially varying buoyancy. Such interference can be reduced by either conducting experiments on thin fluid layers or, better, by going to microgravity conditions. In the latter case, the interface must generally be fixed to remain observable, e.g. by capillary forces in a gap. Buoyancy driven and thermocapillary flows can strongly resemble each other and were consequently confused sometimes in the Bénard instabilities. For the sake of clearness, we further specify them according to the dominant underlying effect as Rayleigh-Bénard (buoyancy driven) and Marangoni-Bénard (thermocapillary driven) instabilities. Table 1-2 lists selected publications on both, structured by orientation of the temperature gradient, the role of gravity, and the type of the interface.

\section{Thermocapillary Convection depending on the Orientation of the Temperature Gradient}

One could consider the thermocapillary convection observed in an experiment as the result of the complex interplay between the different fluid behaviour at and remote from the interface. The 
complexity of this interplay often impedes the unequivocal identification of the phenomena of interest. Thus, various hydrodynamic instabilities can occur, for instance. They denote the abrupt emergence of new convection patterns in a stationary nonequilibrium system as soon as its parameters enter certain ranges [benard1901], [block1956], [joseph1976a], [joseph1976b], [drazin1981], [drazin2002]. One assumes it to exhibit such a behaviour in agreement with the postulated principle of minimum entropy production [glansdorff1971]. Howsoever, both the ranges and their affiliated patterns depend strongly on the detailed system setup. We shall go particularly into the influences of the angle between the predominant temperature gradient and the interface, here.

Already very small temperature gradients can, if oriented properly along an interface, drive a thermocapillary convection [schatz2001]. It occurs at first in a monocellular steady pattern, where the fluid drag at the interface is balanced by laminar counterflows in the adjacent phases. Once the temperature gradient exceeds a critical limit, hydrodynamic instabilities emerge. They exist generally at first as multicellular steady patterns and afterwards as spatio-temporal structures [schwabe1999b], such as the oscillatory flows described in [chun1979]. The flow will eventually become turbulent under even larger temperature gradients.

If the predominant temperature gradient lies normal to the interface, the thermocapillary convection itself occurs immediately as a hydrodynamic instability once the gradient exceeds a certain limit. Already minor temperature fluctuations along the interface will then become self-sustained by the thermocapillary convection that they initiate. Below this instability limit both phases remain quiescent, neglecting the influence of disturbing side-effects. The occurrence of hexagonal Marangoni-Bénard roll-cells [benard1900a] are probably the most familiar example of such instabilities, see Figure 5.

As both cases discussed above show, the thermocapillary convection can occur in quite different shapes, even though it is always driven by the same thermocapillary effect. 


\subsubsection{Solid-Fluid Interfaces}

The interfacial flows occurring at solid-fluid interfaces are behind various familiar phenomena such as the Knudsen effect, thermoosmosis, and thermophoresis. They all have the same appearance under a temperature gradient, no matter whether the fluid phase is liquid or gaseous. Most of the experiments were, however, conducted for gaseous fluids, since they show a more distinct response. Furthermore, the interactions between the gas molecules can be neglected in most cases, which simplifies the modelling. Needless to say that the solid-fluid interactions must be taken into consideration, which usually requires making additional assumptions. Section 2.3.1 will provide an overview of the various modelling approaches.

We shall begin with the Knudsen effect [knudsen1910], [knudsen1927]. Consider a moderately dilute gas in a system of two vessels, $V 1$ and $V 2$. A long capillary connects them as shown in Figure 6 . When both vessels are maintained at the same temperature, $T_{V 1}=T_{V 2}$, the system approaches thermodynamic equilibrium with a homogeneous pressure and density distribution, $P_{V 1}=P_{V 2}$ and $\rho_{V 1}=\rho_{V 2}$. When the vessels are adjusted to somewhat different temperatures, $T_{V 1}=T_{C}$ and $T_{V 2}=T_{H}>T_{C}$, however, a stationary nonequilibrium state establishes. It comes, different from what one might expect at first sight, with a heterogeneous pressure distribution. So-called thermal creep flows, which typically run along the capillary walls from the cold to the hot vessel, lie behind such an unexpected behaviour. These flows produce a pressure difference between the vessels, $P_{V 2}>P_{V 1}$, which in turn drives counterflows of gas at the long axis of the capillary.

There is an inverse relation between the pressure difference and the intensity of the flows. It, in turn, depends, above all, on the collisions of the gas molecules with each other compared to those with the containment walls. Both types of collision affect the system behaviour to a different extent. Their relative influence is described by the Knudsen number, $K n=\lambda / L$ [knudsen1909], where $\lambda$ represents the mean free path of a gas molecule and $L$ a characteristic length, e.g. here the radius of the capillary $d / 2$. In a system with a very small Knudsen number, the thermal creep and its counterflow are spatially separated. They assume a circular thermocapillary convection pattern: While the thermal creep proceeds along the capillary wall, the counterflow concentrates at the capillary axis. Both flows interfere at first stronger if the Knudsen number of the system is increased, e.g. by reducing the capillary radius at constant vessel temperatures and average system 
pressure. As a result, the pressure difference rises. Finally, a so-called "molecular flow regime" is reached for very large Knudsen numbers. Then, the thermal creep and its counterflow cover the entire cross-section of the capillary. Nevertheless, the pressure difference slightly decreases again due to the strongly reduced rate of collisions between the gas particles. It manifests itself also in an additionally occurring phenomenon, called viscous slip (see section 2.3.1). Other influences on the system behaviour are, for instance, the length of the capillary, the mean gas pressure, and the gas viscosity.

In the above setup, a capillary connects both vessels. If we replace it by a porous medium, such as metal foams or cellophane membranes [feddersen1872], [feddersen1873], [reynolds1879], [hanley1965], [hanley1966], a pressure difference still occurs. Apparently, the same processes as in the Knudsen effect remain active for the altered geometry. For historical reasons, one calls specifically the effect behind this difference "thermal transpiration". Both denominations, Knudsen effect and thermal transpiration, are kept despite the blurred transition from capillaries to porous media. Thermal transpiration is also known to accompany the thermoosmosis [denbigh1952a], [denbigh1952b] in membranes depending on the Knudsen number of their pores [rastogi1969].

According to Newton's third law of motion, every force has a "counterforce" of equal magnitude and opposite direction. The walls of a solid must thus feel a "thermocapillary counterforce" which is opposite to the one driving the thermal creep along them. In the phenomena introduced so far, the solid was always fixed and very stiff, so that only the impact of the "thermocapillary force" on the gas can be observed. A mobile solid should, however, be accelerated in the direction opposite to the flux of the adjacent gas. Simply put, the gas goes to the hot region while the solid heads toward the cold one. This behaviour is shown, for instance, by Crookes radiometer [crookes1874], [crookes 1876], [crookes1880], [schuster1880], [pringsheim1883], [sone1991], [sone1994]. It consists of a pivoted wheel of blades in a dilute gas. They absorb incident light on one side, typically covered with soot, and reflect it on the other side, typically covered with a shiny metal. Hence, both sides assume different temperatures when subjected to an infrared light source. Their difference drives creep flows around the edges of the blades. They, in turn, move in the opposite direction, i.e. from hot to cold. In a similar phenomenon, called thermophoresis, particles in aerosols are known to migrate under a temperature gradient towards colder regions [fresnel1825a], [fresnel1825b], [rosenblatt1946], [schadt1961], [jacobsen1965], [derjaguin1966], [keng1966], [byers1969], [ivchenko1971a], [singh1972], [nishio1974], [davis1975a], [davis1975b], [fuchs1982], [stratmann1989], [montassier1990], [chang1995]. 
The phenomena introduced so far for gases occur similarly if the fluid phase is a denser gas or even a liquid. Thus, we find in the literature several reports on the Knudsen effect [knudsen1910] and on the thermophoresis [giddings1970], [mcnab1973], [schimpf1987], [schimpf1989], [shiundu2003]. The basic mechanisms of thermal creep must, therefore, work generally at solid-fluid interfaces although the interactions between the fluid molecules may have more weight than at a solid-dilute gas interface. Nevertheless, this increased weight can affect the creep significantly. We find, in fact, predictions on its inversion in the literature (see section 2.3.2). Howsoever, thermal creep can be practically always expected to occur in a roll cell pattern in denser fluids due to the extremely short mean free path of the molecules (insofar the notion of mean free path in such fluids makes sense at all). Both adjoining phases then generally accelerate in opposite directions at a solid-fluid interface, as pointed out above. Their opposition makes the difference compared to the thermocapillary convection at liquid-fluid interfaces. There, the adjoining phases accelerate in the same direction, i.e. typically from hot to cold. (see section $\mathbf{2 . 3 . 2}$ ). 


\subsection{Heuristic Models of the Interfacial Phenomena}

Let us first review the continuum mechanics, that underlie the different heuristic models, before we will look at them. Continuum mechanics describes the behaviour of a system, e.g. its internal flows, by applying the basic laws of classical mechanics and thermodynamics to macroscopic portions $\Delta V$ of it [mcquarrie2000]. The state variables in the aforementioned laws are first defined as averages over finite intervals of time $\delta t$ and of space $\delta V$ [degroot1960]. Both must be small enough to catch the spatial and chronological variation of the system in consideration and concurrently large enough to obtain reliable state variables. In the continuum hypothesis, the local state variables for any such interval are then assumed to be continuous functions of time and space [landau2003]. One thus obtains for each portion a set of universal balance equations that must still be extended by its interaction with the surroundings [spurk1996]. The interaction with neighbouring portions is included as "fluxes", e.g. of mass, momentum, and energy, with differences of the state variables, e.g. gradients of pressure, temperature, or chemical potential, as driving "forces" [haase1983]. The relationship between the fluxes and forces are characterised by so-called "material equations". They depend on the type of the system. If the processes inside it proceed "moderately" [degroot1960], these equations are a linear combination of forces, with phenomenological coefficients whose matrix must be symmetrical according to Onsager's reciprocity relations [onsager1931a], [onsager1931b].

Continuum mechanics can describe a great variety of situations in practice. Suitable boundary conditions or material equations are typically gained empirically, for instance the frequently used no-slip condition at interfaces or the Cauchy-Poisson material equation [spurk1996]. While this empirical approach has proven itself for separate phases, it is arduous for most multiphase systems. Their interfacial phenomena are hardly accessible to experiments. Instead, one resorts to heuristic assumptions. They supplement the few consolidated experimental findings so as to incorporate these phenomena. Hitherto, a variety of such phenomenological models has emerged. They are at most satisfactory. The simplest ones (see section 2.2.1) consider interfaces as two-dimensional entities through special boundary or matching conditions, such as the no-slip condition for instance. More advanced models account for the interface as a three dimensional region by means of extended material equations (see section 2.2.2). Let us, however, take a closer look at the linear 
momentum balance first, following [spurk1996]. It specifies, eventually, the conditions for the occurrence of flows in general and thus also the creep and thermocapillary ones considered here.

We can establish a linear momentum balance for each of the finite space intervals, $\delta V$, considered within the continuum hypothesis. The total force $\mathbf{f}$ on every one of them must always equal its temporal and convective change in momentum:

$\bar{\rho}\left[\frac{\partial \mathbf{v}}{\partial \mathrm{t}}+(\mathbf{v} \cdot \nabla) \mathbf{v}\right]=\mathbf{f}$

Here, $\rho$ represents the fluid density, $\mathbf{v}$ the velocity, and $\mathbf{f}$ the local volume force. In practice, one can often clearly subdivide the latter into two contributions, depending on the dimensions of its reference interval $\delta V$ :

$\mathbf{f}=\mathbf{f}^{\text {int }}+\mathbf{f}^{\text {ext }}$

The longer ranged or "external force", $\mathbf{f}^{\text {ext }}$, such as gravitation for instance, affects all space intervals in a system to almost the same extent. On the other hand, there is the short ranged or "internal force", $\mathbf{f}^{\text {int }}$, for instance, due to the molecular interactions. Since it acts only between neighbouring space intervals, it varies typically much stronger within a system. One then rewrites the "internal force" as the divergence of a locally applicable stress tensor $\mathbf{S}$, to be explained in more detail below. Thus, the linear momentum balance reads

$\bar{\rho}\left[\frac{\partial \mathbf{v}}{\partial \mathrm{t}}+(\mathbf{v} \cdot \nabla) \mathbf{v}\right]=\nabla \cdot \mathbf{S}+\mathbf{f}^{e x t}$

Equation (2-1) has the so-called differential form. Its integration over a larger system portion of volume $\Delta V>>\delta V$ results in the so-called integral form:

$$
\iiint_{\Delta V} \rho\left[\frac{\partial \mathbf{v}}{\partial \mathrm{t}}+(\mathbf{v} \cdot \nabla) \mathbf{v}\right] \mathrm{d} V=\iiint_{\Delta V} \mathbf{f}^{e x t} \mathrm{~d} V+\iint_{\Delta S} \mathbf{n} \cdot \mathbf{S} \mathrm{d} S .
$$

Here, $\mathbf{n}$ represents the local normal vector on the surface, $\Delta S$, of the portion (see Figure 7).

The stress tensor contains the stress vectors, s, on three macroscopically small cut-surfaces perpendicular to the coordinate axes at the location r, see Figure 8 [goldstein2002]. We shall symbolise the elements of the stress tensor by the letters $S_{i j^{\prime}}$ where the first subscript $i^{\prime} \in\{x, y, z\}$ represents the component of the stress tensor on the cut-surface $\delta S_{j^{\prime}}$ perpendicular to the axis $j^{\prime} \in\{x, y, z\}$ in the second subscript: 


$$
\mathbf{S}=S_{i^{\prime} j^{\prime}}=\left(\begin{array}{lll}
S_{x x} & S_{y x} & S_{z x} \\
S_{x y} & S_{y y} & S_{z y} \\
S_{x z} & S_{y z} & S_{z z}
\end{array}\right)
$$

The three elements with identical subscripts $S_{i^{\prime}=j^{\prime}}$ constitute normal stresses and the other six elements $S_{i^{\prime} \neq j^{\prime}}$ shear stresses. In an equilibrium one-phase system, the stress tensor is diagonal while its normal stresses equal the negative system pressure. Such a simple relation does not hold in other systems anymore. One then often simply averages the normal stresses. Their negative average serves, at least, as a crude measure for the local pressure level:

$$
\Pi=-\frac{S_{x x}+S_{y y}+S_{z z}}{3}
$$

In a quiescent fluid with constant total momentum, shear flows occur generally only if the forces on the right side of the momentum balance (2-1) are non-zero. This includes also the interfacial flows considered here. Since such flows, in particular, occur also in the absence of external forces, as stated in chapter 2.1, internal forces must be the cause. They exist according to equation (2-1) if the divergence of the stress tensor,

$$
\mathbf{f}^{\text {int }}=\nabla \cdot \mathbf{S},
$$

is non-zero. Interfaces are, however, often thinner than the range of the interatomic forces. This makes the above conception of the internal forces as surface forces appear questionable for the small subvolumes that are necessary to catch the spatial variation of the interfacial regions. Nevertheless, we shall stick to this conception here since it is widely spread on the one hand and can, on the other hand, be shown apply at least formally correctly in the case of our equilibrium interfacial systems (see section 4.2.1). Section 2.3 introduces explanations of how such a non-zero divergence can arise, using a solid-dilute gas interface as an example. Later, in our actual studies, we shall estimate the internal forces involved in the thermocapillary effect by taking advantage of their relation to the stress tensor (see section 5.5.2).

For the sake of completeness, we shall also give the other balance equations here, as found in [spurk1996]. The internal and external forces also participate in an angular momentum balance. It is analogous to the one of the linear momentum above and requires the symmetry of the stress tensor: $\mathbf{S}=\mathbf{S}^{\mathrm{T}}$

Moreover, there is the mass conservation law. Its local form reads: 
$\left(\frac{\partial \rho}{\partial t}\right)+\bar{\rho} \nabla \cdot \mathbf{u}+\mathbf{u} \cdot \nabla \bar{\rho}=0$

Equations (2-2), (2-3), and (2-4), constitute together the so-called mechanical balance equations. One additionally uses the co-called thermodynamic ones to describe a flow. We shall specify them only very generally here:

$$
\begin{aligned}
& \frac{\partial e}{\partial t}+(\mathbf{u} \cdot \nabla) e=\delta \dot{w}+\delta \dot{q} \text { and } \\
& \frac{\partial s}{\partial t}+(\mathbf{u} \cdot \nabla) s=\delta \dot{s}_{\mathrm{rev}}+\delta \dot{s}_{\mathrm{irrev}}
\end{aligned}
$$

They consider the local energy, $e$, and entropy, $s$. The left-hand sides represent the chronological and convective variation of the applicable balanced state function. Both add to its other fluxes across the boundaries of the considered reference interval $\delta V$. Entropy, in particular, can moreover be produced. Its fluxes, $\delta \dot{s}_{\text {rev }}$, are, different from its production rate, $\delta \dot{s}_{\text {irrev }}$, reversible. One typically distinguishes the exchanged energy fluxes depending on the way of transportation. Heat, $\delta \dot{q}$, is diffusively and work, $\delta \dot{w}$, non-diffusively transported. 


\subsubsection{Heuristic Boundary Conditions}

Numerous interfacial models have emerged, based on experimental studies of the thermocapillary effect and of other interfacial phenomena. The simplest models treat the interface as a twodimensional entity, as suggested by Young [young1805], Laplace [laplace1880], and Gauss [gauss1830]. They describe each phase separately by the established continuum mechanical theories. The processes at the interface, itself, are simply accounted for through suitably chosen boundary or matching conditions [levich1962]. Such an approach considers specifically a liquidfluid interface as a "membrane". It feels a measureable tension which depends, among other things, on the local temperature. We obtain a simple hydrodynamic boundary condition by assigning the interfacial tension gradient to the lateral components of the stress tensor, as in [levich1962]. More sophisticated formulations for boundary conditions have emerged up to now. They consider also other interfacial phenomena of relevance, for instance, the deformability of the interfaces and its effect on the interfacial tension or on the interfacial rheology [scriven1960a]. All in all, such boundary conditions have been successfully used for a wide range of situations, in particular for the prediction of the thermocapillary convection [pearson1958], [scriven1964], [villers1992]. However, all these models must break down when the processes at the interfaces are to be described on length-scales smaller than its thickness, such as in coalescence or in thick near-critical interfaces.

Let us state here for the sake of completeness that the thermal creep at solid-dilute gas interfaces is well modelled by using the concept of an interfacial tension, although it cannot be measured there. Instead the relevant phenomenological boundary conditions are therefore deduced from more fundamental theories. We address such deductions in section $\mathbf{2 . 3}$, because they are also of relevance for the thermocapillary effect. 


\subsubsection{Heuristic Material Equations}

So-called "diffuse interface models" account, as suggested by Poisson [poisson1831], Maxwell [maxwell1876], and Gibbs [gibbs1875], for the finite thickness of the interface by treating it as a heterogeneous but continuous region. The processes in it are described by suitable extensions of the well-known thermo- and hydrodynamic theories [anderson1998]. Thus, one assumes, for instance, that their variables can unambiguously specify all local states inside the system on the required length and time scales, as described previously in section 2.2. Such a specification is, however, complicate at the interface due to the strong variation of the "interfacial states". It entails, among other things that the state of an interfacial subdomain depends not only on its state variables but also on its interactions with the surrounding subdomains. The various "diffuse interface models" try to take these interactions into account by supplementing the familiar balance and material equations (see section 2.2) with contributions from the neighbouring state variables [korteweg1901], [dunn1985], [piechor2008]. Think, for instance, of the energy balance or the material equation of the stress tensor. They are frequently expanded by terms that involve gradients of the density or of the composition. Sometimes, even new state variables, e.g. a phase field, are introduced for convenience.

One applies diffuse interface models to interfacial systems in and out of equilibrium. We shall take a closer look at the former case here to clarify some terms for later use. The description simplifies significantly in equilibrium due to the irrelevance of the hydrodynamic properties. Concurrently, the thermodynamic state variables can be classified into "field properties" and "intensive densities" [rowlinson2002]. We denote by "fields" those properties that have, in agreement with the conditions for thermodynamic equilibrium, a homogeneous distribution throughout the entire system. Think, for instance, of the temperature or of the chemical potentials of the individual components. The "intensive densities", on the other hand, vary. They are the extensive thermodynamic properties that had to be redefined locally by relating them to another extensive property, typically on a molar or molecular basis. Their variations are the strongest at the interface and converge to zero deep in the phases. Hence, we can consider each phase, suitably remote from the interface, as a "bulk phase" whose state persists after isolation from the rest of system. The interface, itself, consists of a sequence of local stationary states that vary continuously from one "bulk phase" to the other. Each of these states can, however, only exist localised between the bulk phases under the influence of its 
neighbouring states - but not in an autonomous and homogeneous "bulk phase". This consideration will be fundamental for the interpretation of our computer simulations.

Van der Waals Theory was presumably the first "diffuse interface model" for vapour-liquid interfaces in unary equilibrium systems [vanderWaals1893], [rowlinson1979]. Cahn and Hilliard reformulated and extended this theory [cahn1958]. For nonequilibrium interfacial systems a series of models was developed that are, for example, reviewed in [anderson1998]. Diffuse interface models may generally be used for thin interfaces away from the critical point. Near the critical point, when the coherence length of fluctuations in the fluids surmounts that of the molecular interactions, the local definitions of some properties, in particular the thermodynamic functions, become increasingly inapplicable [rowlinson2002]. As a result, diffuse interface models underestimate the thickness of the interface in such cases, which leads to an overestimation of the phase separation.

All "extended models" fail to account correctly for the experimentally measurable convection that spreads from the interface into the phases [pertler1995], [davis1987], [cramer1995], [schatz2001]. Similarly, these models also fail to reproduce other interfacial phenomena such as interfacial rheology, diffusocapillary effects, and electrocapillary effects [stuke1961], [sawistowski1971], [sherwood1975], [levich1969]. 


\subsection{Fundamental Models for the Interfacial Phenomena}

We can apply more fundamental theories than e.g. the phenomenological continuum mechanics from section 2.2 to describe the behaviour of a system. Quantum-mechanics is the most fundamental one [mcquarrie1997], [dirac1999]. The molecular theories of gases and liquids constitute its classical limit [kennard1938], [hirschfelder1966], [chapman1970]. Since such theories are deterministic by nature, we must know the initial microstate and the external constraints of a given system entirely in order to predict its exact evolution. Observables, i.e. in principle measurable quantities, are averages over such evolutions. However, the initial microstates, and partially even the boundary conditions, remain practically indeterminable. Instead, the fundamental theories were reformulated with a statistical character by making additional assumptions. This major contribution to modern science is due to Ludwig Boltzmann (1844-1906) who based his consideration on classical Newtonian mechanics [mcquarrie2000], [landau2007]. The underlying concepts were later on shown to be entirely compatible with quantum mechanics. A macroscopically unambiguously specified system state is represented by a probability distribution function over several microstates. Studying its evolution thus amounts to studying the one of the system. We then obtain its macroscopic observables by considering all microstates with their concomitant probabilities.

The boundary conditions and material equations for the continuum mechanical description of matter can in principle, and under special circumstances also in practice, be derived by using the fundamental theories above. Such "fundamental approaches" have proven to be successful mainly for simple molecules and dilute gases. In particular, the different explanations of the creep flows at a solid-dilute gas interface under a temperature gradient draw our attention, here. They strongly resemble, when given small Knudsen numbers $K n \rightarrow 0$, the thermocapillary flows at a liquid-liquid interface under a similar gradient. There is only one major difference. The adjacent phases accelerate typically in different directions at solid-dilute gas and liquid-liquid interfaces (see chapter 2.1). Nevertheless, one feels intuitively a relationship between both phenomena, which is why we will take a closer look at the explanations of thermal creep, below. Since they all use the kinetic theory of gases, we shall first review it and its definition of the stress tensor. James Clerk Maxwell (1831-1879) was presumably the first to derive a macroscopic relation for thermal creep. In section 
2.3.1, we will briefly explain his derivation. Hitherto, more advanced models have been proposed based on it, see section 2.3.2.

\section{Kinetic Theory of Gases}

The kinetic theory of gases specifies their microscopic state through the particle distribution function $\Phi$, see e.g. [kennard1938], [sone2007] for details. In an atomic gas, this function describes the probability of finding at the time $t$ a particle with the velocity $\mathbf{u}$ at the coordinate $\mathbf{r}: \Phi(\mathbf{r}, \mathbf{u}, t)$. If the gas is sufficiently dilute, $\Phi$ can be rewritten as the product of the local density, $\rho(\mathbf{r}, t)$, multiplied by the particle velocity distribution function, $f(\mathbf{u}, t)$ :

$\Phi(\mathbf{r}, \mathbf{u}, t)=\rho(\mathbf{r}, t) f(\mathbf{u}, t)$.

It has a Maxwell-Boltzmann type in the special case of an isolated bulk gas in thermodynamic equilibrium,

$f_{0}(\mathbf{u})=A e^{-\beta^{2}|\mathbf{u}|^{2}}$

where $A$ is a normalisation constant and $\beta$ results from the particle mass, $m$, Boltzmann's constant, $k_{B}$, and the temperature, $T$, according to the relation

$$
\beta^{2}=\frac{m}{2 k_{B} T}
$$

\section{Microscopic Definition of the Stress Tensor in a Dilute Gas}

There are generally two contributions, a kinetic one, $\mathbf{K}(\mathbf{r})$, and one, $\mathbf{I}(\mathbf{r})$, from the interatomic forces, to the microscopic definition of the stress tensor $\mathbf{S}(\mathbf{r})$. Dilute gases have only a significant kinetic contribution. It represents the average flux of $i^{\prime}$-momentum, $i^{\prime} \in\{x, y, z\}$, per unit time and area across the three macroscopically small cut surfaces, $\delta S_{j}$, [kennard1938] (see Figure 9 on the left side). They lie perpendicular to the coordinate axes, $j^{\prime} \in\{x, y, z\}$, and intersect at the location $\mathbf{r}$. The flux, itself, happens through the movement of the particles. They carry their momentum with them whenever they cross one of these cut surfaces. Such a crossing happens in the case of every particle, $i$, whose location vector, $\mathbf{r}_{i}$, is part of them, i.e. $\mathbf{r}_{i} \in \delta S_{j^{\prime}}$. We obtain the total flux by adding or by withdrawing the $i^{\prime}$-momenta of the particles, depending from which side they come: 
$K_{i^{\prime} j^{\prime}}=-\frac{\left\langle\sum_{\mathbf{r}_{i} \in \delta S_{j^{\prime}}} \operatorname{sgn}\left(\mathbf{u}_{i} \cdot \mathbf{e}_{j^{\prime}}\right) \cdot m_{i}\left(\mathbf{u}_{i} \cdot \mathbf{e}_{i^{\prime}}\right)\right\rangle}{\delta S_{j^{\prime}} \delta t}$.

It occurs in the sign of the scalar product between the particle velocity, $\mathbf{v}_{i}$, and the unit vector, $\mathbf{e}_{j}$, perpendicular to the cut surface, $\delta S_{j^{\prime}}$. The momenta carried by the particles are their mass, $m_{i}$, multiplied by the scalar product of their velocity, $\mathbf{u}_{i}$, and the unit vector in $i^{\prime}$-direction, $\mathbf{e}_{i^{\prime}}$. We additionally multiply the kinetic contribution with -1 to make it compatible with the hydrodynamic definition of the stress tensor. It is, according to hydrodynamics, a compilation of the forces per unit area on cut surfaces whose normal vectors point in the positive directions of the coordinate axes (see section 2.2).

In practice, it is often desirable to calculate the stress tensor from the particle distribution function, $\Phi$, rather than tracking the motion of the individual particles. One, therefore, rewrites the molecular definition: Not just the particles crossing a cut surface are considered anymore, but all particles inside a small macroscopic prism of the height $2 \delta \alpha_{j^{\prime}}$ drawn symmetrically on both sides of this surface [heinz2005] (see Figure 9 on the right side). A given particle, $i$, with the velocity, $\mathbf{u}_{i}$, and an unknown position, $\mathbf{r}_{i}$, inside the prism crosses the surface during a brief time interval, $\delta t$, only with a probability of $\left(\mathbf{u}_{i} \cdot \mathbf{e}_{j^{\prime}}\right) \delta t / 2 \delta \alpha_{j^{\prime}}$. It accounts for the fact, that the perpendicular velocity component $\left(\mathbf{u}_{i} \cdot \mathbf{e}_{j^{\prime}}\right)$ may be directed towards or away from the surface, through the factor 2 . Using this probability, we obtain the momentum transferred by summing the particle momenta, weighted by the corresponding probabilities, of all particles inside the prism. Additional normalisation by $\delta t$ and the area of $\delta S_{j^{\prime}}$ yields the momentum flux density. Its average constitutes a computationally easy to handle definition of the kinetic contribution to the stress tensor:

$K_{i^{\prime} j^{\prime}}=-\frac{\left\langle\sum_{i \in \delta V=2 \delta S_{j^{\prime}} \delta \alpha_{j^{\prime}}} m_{i}\left(\mathbf{u}_{i} \cdot \mathbf{e}_{i^{\prime}}\right)\left(\mathbf{u}_{i} \cdot \mathbf{e}_{j^{\prime}}\right)\right\rangle}{2 \delta S_{j^{\prime}} \delta \alpha_{j^{\prime}}}$.

Equation (2-5) can be further rewritten as an integral by means of the local densities and the particle velocity distribution function. Particularly in a pure substance or, at least, a mixture of particles with equal masses, $m$, the stress tensor simply amounts to

$$
K_{i^{\prime} j^{\prime}}=-\rho \cdot m \cdot \iiint\left(\mathbf{u} \cdot \mathbf{e}_{i^{\prime}}\right) \cdot\left(\mathbf{u} \cdot \mathbf{e}_{j^{\prime}}\right) \cdot f(\mathbf{u}) \mathrm{d} \mathbf{u} .
$$

Here, $\rho$ is the particle density and $f(\mathbf{u})$ the particle velocity distribution function [kennard1938] of the gas. We can easily compute the integral in thermodynamic equilibrium. An isolated bulk gas then has normal stresses of 
$K_{x x}=K_{y y}=K_{z z}=-\rho k_{B} T$.

This equation holds approximately also in thermodynamic nonequilibrium if the transport processes proceed moderately in the sense of continuum mechanics (see chapter 2.2). 


\subsubsection{Maxwell's Explanation for the Thermal Creep}

Consider, as an example, a system of a pure semi-infinite quiescent dilute gas which is in contact with a fixed semi-infinite solid. Its surface lies perpendicular to the $z$-axis at $z=0$ (see Figure 10). Both the gas and the solid may be subject to the same temperature $y$-gradient $\partial T / \partial y>0$, while other external influences remain absent. According to Maxwell, thermal creep arises in such a system due to the special two-dimensional heterogeneity of the gas-solid interfacial region [maxwell1879]. The difficulties in studying such and other interfaces were described before (see section 1.1). We can, however, at least infer on this region by looking at two particular cut-outs of the system: one directly at and the other one far remote from the interface. They constitute limiting cases which are relatively well understood in terms of the kinetic theory of gases.

Let us look at the gas away from the interface first. There, a bulk like behaviour establishes, with heterogeneity only in $y$-direction. Given small temperature gradients, the particle velocity distribution function can most conveniently be considered as a sum of the Maxwell-Boltzmann velocity distribution function, $f_{0}$, and a bias, $f_{1}$ :

$f=f_{0}+f_{1}$.

It must be antisymmetric in $y$-direction in order to make the centre of mass $y$-velocity zero,

$$
\int_{-\infty-\infty-\infty}^{\infty} \int_{-\infty}^{\infty} u_{y} f(\mathbf{u}) \mathrm{d} \mathbf{u}=0
$$

and thus cannot contribute to the stress tensor in equation (2-6). We obtain an estimate of the bias from more advanced treatments of such heat conducting bulk gases [kennard1938]:

$$
f_{1}=C u_{y}\left(\frac{5}{2}-\beta^{2}|\mathbf{u}|^{2}\right) e^{-\beta^{2}|\mathbf{u}|^{2}} .
$$

Here, $C$ is a normalisation constant and, $u_{y}$, the particle velocity component in $y$-direction.

We shall now take a closer look at the second limiting case. An adsorption layer forms at the interface even for very low gas pressures. Its local density thus clearly exceeds the one of the bulk gas. Incident particles interact for different amounts of time with the adsorption layer and the solid until they are reflected back. The longer this interaction takes for a certain particle, the more its 
velocity of reflection will be randomised. We can distinguish two limiting cases again: The immediate specular reflection and the diffuse reflection after a long interaction period during which the particle becomes a constituent of the adsorption layer. In the diffuse case, the velocity of reflection is purely random, with probabilities according to the local particle velocity distribution function at the solid. A reflection has typically, at least, a partially diffuse character. We can quantify it, for instance, by the average percentage, called accommodation coefficient, $a$, of the rather diffuse reflections in all reflections [maxwell1879]. Given such a coefficient larger zero, the particle velocity distribution function remote from the interface deviates from a more symmetric one right at it. Computing the transition between both limiting cases is an intricate task to accomplish which requires eventually solving the Boltzmann equation (see section 2.3.2). We can, however, anticipate that this solution must be a continuous function of space, with increasing rates of change in approaching the solid. This gives rise to spatially variable shear stresses $S_{y z}$ in $z$ direction. They come with a non-zero divergence of the stress tensor and thus drive the thermal creep.

In order to estimate the creep velocity, Maxwell made the rather bold assumption that the density and the particle velocity distribution function $f(\mathbf{u})$ remain homogeneous up to the solid, where all incident particles are reemitted diffusely, i.e. $a=1$ [maxwell1879]. Given a quiescent gas, the distribution function there is thus simply the one of the incident particles, $f_{\text {inc }}(\mathbf{u})$, superimposed with the one of the reflected particles, $f_{\text {rep }}(\mathbf{u})$ :

$f=f_{\text {inc }}+f_{\text {rep }}=\left(f_{0}+f_{1}\right)\left(v_{x}<0, u_{y}, u_{z}\right)+f_{0}\left(u_{x}>0, u_{y}, u_{z}\right)$

This superimposed function entails a loss of $y$-momentum

$$
\begin{aligned}
& K_{y z}=-\rho \int_{-\infty-\infty}^{\infty} \int_{-\infty}^{\infty} \int_{y}^{\infty} u_{y} u_{z} f(\mathbf{u}) \mathrm{d} \mathbf{u}= \\
& =-\rho \iint_{-\infty-\infty}^{0} \int_{-\infty}^{\infty} \int_{y}^{\infty} u_{y} u_{z}\left(f_{0}(\mathbf{u})+f_{1}(\mathbf{u})\right) \mathrm{d} \mathbf{u}-\rho \iint_{0}^{\infty} \int_{-\infty-\infty}^{\infty} u_{y} u_{z} f_{0}(\mathbf{u}) \mathrm{d} \mathbf{u}= \\
& =-\rho \int_{-\infty-\infty}^{0} \int_{-\infty}^{\infty} \int_{y}^{\infty} u_{y} u_{z} f_{1}(\mathbf{u}) \mathrm{d} \mathbf{u}= \\
& =\rho C \int_{-\infty-\infty}^{0} \int_{-\infty}^{\infty} \int_{y}^{\infty} u_{y}^{2} u_{z}\left(\frac{5}{2}-\beta^{2}|\mathbf{u}|^{2}\right) e^{-\beta^{2}|\mathbf{u}|^{2}} \mathrm{~d} \mathbf{u}= \\
& =\frac{\pi}{8} \frac{\rho C}{\beta^{6}}
\end{aligned}
$$


from the adjacent gas layer to the solid. As a result, both accelerate relative to each other in opposite $y$-directions. Since the solid is fixed as stated above, the relative acceleration applies also in absolute terms to the adjacent gas layer. Its incipient motion in positive $y$-direction affects the superimposed particle velocity distribution function thus balancing the loss of $y$-momentum to the solid. Concurrently, further remote layers of gas are as well accelerated due to its viscosity. Eventually, a uniform thermal creep $y$-velocity, $v_{T C}$, establishes. It amounts to

$$
v_{T C}=\frac{3}{4} \frac{\tilde{\eta}}{\rho T} \frac{\partial T}{\partial y}=\frac{3}{4} \frac{\tilde{\eta} R}{P M} \frac{\partial T}{\partial y},
$$

based on further considerations [kennard1938]. Here, $\tilde{\eta}$ is the viscosity, $P$ the pressure, and $M$ the molar mass of the gas, while $R$ represents the universal gas constant.

If the thermal creep cannot develop uniformly, as is typically the case in practice, velocity gradients will occur in the gas. They can give rise to additional slip flows along the solid for small Knudsen Numbers. Maxwell explained this phenomenon, called viscous slip, similar to the thermal creep above, by the differences between the velocity distribution functions of the particles incident on and reemitted from the adsorption layer [kennard1938], [maxwell1879]. Thus, the viscous slip velocity, $v_{V S}$, right at the gas-solid interface must be directly proportional its normal velocity gradient $v_{V S}=-\zeta_{V S} \frac{\partial v_{y}}{\partial z}$

where $\zeta_{V S}$ is the slip coefficient. We can simply add the velocities of viscous slip and thermal creep in a first approximation,

$v=v_{T C}+v_{V S}$

Given small Knudsen numbers, Maxwell's expressions for thermal creep and viscous slip have proven themselves in principle as boundary conditions for the phenomenological description of dilute gases in contact with a solid. Thus, particularly the thermophoresis from section 2.1 .2 can be predicted with satisfactory accuracy. Epstein's well-known formula for the thermophoretic velocity of the objects in a suspension [epstein1929], for instance, builds on a continuum mechanical model with Maxwell's expression for thermal creep as boundary condition. Even more modern models of thermophoresis still use Maxwell's expressions [brock1962]. 


\subsubsection{Advanced Models for the Interfacial Phenomena}

Hitherto, Maxwell's treatment of thermal creep was advanced to obtain more accurate predictions for a broader range of situations. In particular the assumption of a constant particle distribution function, $\Phi(\mathbf{r}, \mathbf{u}, t)$, up to the solid is abandoned. Instead one solves the mathematically difficult Boltzmann equation [sone2007]. It describes the spatial and chronological variation of the particle distribution function. All solutions obtained indicate asymptotically increasing creep velocities remote from the solid. Their limiting values exceed Maxwell's result (see equation (2-8)) by approximately 50\% [sone1966], [sone1968b], [loyalka1971a], [ohwada1989a].

In addition to the solution of the Boltzmann equation, the research concentrates on how the interactions of the bulk gas particles with the adsorption layer affect the creep velocity. Given small Knudsen numbers, $K n \rightarrow 0$, it has a maximum for entirely diffuse reflections $(a=1)$ and decreases towards Maxwell's derived creep velocity the more the reflections are specular $(a=0)$ [deryagin1967], [yalamov1968], [abramov1970], [loyalka1971b], [loyalka1971c], [onishi1972], [loyalka1975], [bakanov1977c], [ivchenko1993]. More sophisticated treatments even characterise the collisions by several accommodation coefficients [brock1962], e.g. a thermal one, $a_{t}$,

$$
a_{t}=\frac{\hat{E}_{i}-\hat{E}_{r}}{\hat{E}_{i}-\hat{E}_{w}},
$$

and a momentum related one, $a_{m}$,

$$
a_{m}=\frac{G_{i}-G_{r}}{G_{i}}
$$

Here, $\hat{G}_{i}$ and $\hat{G}_{e}$ represent the tangential components of the momentum, $\hat{G}$, and the kinetic energy flux density, $\hat{E}$, carried either by the incident or the reflected particles (subscripts $i$ and $r$ ). $\hat{E}_{w}$ stands for the energy flux density that would be emitted if the particles came from an equilibrium bulk gas at the temperature right in front of the solid.

One must keep in mind, however, that the different characterisations of the particle reflections are merely workarounds in the conception of the adsorption layer. Little is known about its true processes involved [darling1995], [gross1998], [kroes1999]. Moreover, it becomes increasingly 
unrealistic to assume distinct collisions with the adsorption layer for higher gas or even liquid densities. Nevertheless, the fundamental models above can describe, either by themselves or in combination with macroscopic theories such as continuum mechanics, the behaviour of various gassolid systems, e.g. the Knudsen effect along a capillary [derjaguin1965], [waldmann1966], [waldmann1967], [sone1968a], [loyalka1969], [skakun1974], [ohwada1989b] or the thermophoretic velocity of an object [deryagin1961], [derjaguin1962a], [deryagin1962b], [mason1962] [fuchs1964], [derjaguin1965], [brock1967a], [brock1967b], [bakanov1976], [gorelov1976], [bakanov1977a], [bakanov1977a], [brock1968], [springer1970], [ivchenko1971b], [beresnev1993], [poddoskin1982], [bakanov1991], [bakanov1992]. In more advanced treatments of such systems with a variable normal temperature gradient along the interface [sone1972], [beresnev1985], [aoki1989], [ohwada1992], [bakanov1992], [takata1995], as e.g. in the thermophoresis of highly heat conductive objects, thermal creep can even invert, i.e. the adjacent gas moves from hot to cold along the solid as in the thermocapillary convection. Although experimental evidence is still missing [bakanov1995], this could hint at a relationship of nature whatsoever between the thermocapillary flows at liquid-fluid and the creep at solid-fluid interfaces (see chapter 2.1). 


\subsection{MD simulations of the Thermal Creep at Solid-Fluid Interfaces}

Wold and Hafskjold [wold1999] have analysed the thermal creep of a binary mixture in a carbon slit pore by using NEMD simulations. See part 3 for the details about this method. The authors' system setup resembles pretty much the one used to explain the Knudsen effect in section 2.1.2. They chose, however, the system constraints such that the fluid was either liquid or supercritical. Nevertheless, the creep flows occur in the simulations as known for gases, when the pore is made subject to an axial temperature gradient. To further study the influences of the flows, the authors simulated several slit pores of different widths, each with six different wall potentials: 100\%, 30\%, and 14\% of (i) a full 10-4-3 Steele potential [steele1974], which is attractive and repulsive, and (ii) an altered 10-4-3 Steele potential, truncated at its first minimum and shifted to be purely repulsive. Such potentials entail, by nature, specular wall collisions. To implement diffuse wall collisions instead, the reflected particles were assigned random wall-parallel velocity components. Their probabilities agree with the Maxwell-Boltzmann distribution at the applicable local temperature.

The authors assess the intensity of the thermal creep on the basis of three properties: The local com velocity distribution, the attenuation of the component separation due to the Ludwig-Soret effect in the slit pore (compared to that in the bulk), and the surface tension gradient along the wall. Provided that the slit pore is sufficiently wide, the expected laminar and stationary roll cells occur with thermal creep along the wall from cold to hot for the 10-4-3 Steele potentials (i). The convection is the strongest for the full potential and weakens the more the potentials are reduced. Using the purely repulsive 10-4-3 Steele potentials (ii), however, the sense of rotation of the roll cells inverts, with "thermal creep" from hot to cold. Its inversion comes with one in the gradient of the interfacial tension. We discussed previously how the nature of the wall collisions affects the thermal creep (see section 2.3). The found inversion suggests that the interatomic forces between the wall and the fluid particles can play an important role as well. In fact, we shall determine a similar mean force field on the particles at our liquid-liquid interfaces (see section 5.1). Later, different simplified variants of it are implemented to establish a relationship between thermal creep and thermocapillary convection (see section 5.6.2). 


\section{Theoretical Background of MD Simulation}

It is the aim of statistical physics to explain, and ultimately predict, the macroscopic behaviour and properties of a given "physical system", based on the microscopic properties of its constituent molecules [hill1987], [mcquarrie2000]. This requires that these properties can be expressed in a suitable way; the expression is most often done in form of a "microscopic model" under characteristic assumptions [bopp2008]. The most frequent one is that matter consists of individual particles (this term is taken here to mean either atoms, groups of atoms, or molecules) that interact via "interaction potentials". In classical statistical mechanics one assumes, moreover, that the particles obey the equations of motion of classical mechanics (Newton's equation). The characteristic assumptions introduced up to here are reasonable ones for chemical systems, i.e. matter with moving masses larger than the mass of the hydrogen atom and temperatures of a few hundred Kelvin, if chemical reactions, i.e. the transformation of one species into another, are neglected [chandler1987].

Any "physical system" amenable to these assumptions can thus, in principle, be "translated" into a corresponding "model system" described mathematically by the coupled Newton equations of motion for the particles. The number and formulation of these equations are to be chosen so as to represent, as best as one can, the specific "physical system" and its interactions with the surroundings (i.e. parts of the world not explicitly included in the system). This "translation" requires further so-called "modelling assumptions" that can be crudely subdivided into three parts: A "molecular model" for the individual particles is necessary that captures their geometry (i.e. space-filling properties) and mass distribution. Furthermore, one needs a description of the interactions between all particles inside the system. This, the part most difficult to obtain, is, colloquially, also known as the "force field". Force-field development for specific systems of chemical, biochemical, and other interests is presently one of the main endeavours of theoretical chemistry [stone2002]. For our purposes here, however, the simplest assumptions as to these interactions will do. Finally, the influences of the surroundings, the so-called "boundary conditions" or "constraints", must be emulated within the governing equations of the "model system".

According to statistical physics, the complete information concerning the chronological evolution of the "model system" is available from its "full phase space trajectory", i.e. the knowledge of all 
particle positions and momenta at all times. Obtaining it is, of course, impossible for many reasons; one of them being that the initial molecular state of the relevant "physical system" cannot be determined. Moreover, the governing system of equations for virtually all situations encountered in experiments would simply be too complex [landau2007]. These problems can nevertheless be tackled by statistical approaches which are eponymous for the branch of statistical physics. In this part we shall look at two of them.

The traditional statistical mechanical approach manages to capture parts of the "molecular information" contained in the "full phase space trajectory" of the "model system" from its related ensemble, see part 9. Such an ensemble constitutes a hypothetical infinite set of microscopically different entities of the "model system" that are all consistent with the macroscopic state of the given "physical system", i.e. its external constraints. This capturing works, however, only in "simple" cases, i.e. if the interactions can be expressed in very simple mathematical forms.

The simulation approach, on the other hand, acknowledges that it is not possible to generate the full phase space trajectory. Instead, one settles for generating a representative sample. MD simulations, in particular, compute a section of the phase space trajectory for the "model system". In doing so, additional assumptions, we shall call them "technical simplifications", are usually introduced to solve the equations of motion efficiently. The thus obtained simplified "model system" shall be denoted henceforth as the "simulation system". As long as the computed section is representative, the results can be transferred to the "model system" and ultimately to the guiding "physical system". Section 3.2 below gives more details on the MD simulations, their simplifications, and their representativeness.

Even the "molecular information" contained in the sample phase space trajectory is far too extensive. Statistical physics, therefore, proceeds through suitable averaging procedures with respect to the observables of interest, mostly macroscopic ones, e.g. variables known from reversible or irreversible thermodynamics and hydrodynamics [keizer1987]. It is at the core of statistical mechanics to devise these averaging procedures. We shall decisively draw on this concept for our analysis of the various systems simulated in this work. 
In order to ease the understanding of statistical physics, however, we shall put here the cart before the horse and look first at the analysis of the phase space trajectory in section 3.1. The discussion of the actual MD simulation method to obtain such phase space trajectories shall be postponed to section 3.2. 


\subsection{Analysis of the Phase Space Trajectory}

For the sake of the argument, we shall assume in this section that at least a representative sample of the "phase space trajectory" of a "model system" is known and look into its analysis. If the trajectory was obtained from MD simulations, some minor differences must be taken care of. This is explained in section 3.2. Let us recall some terms first, before we will address the analysis. By "phase space" we denote a multidimensional space spanned by the positions and the momenta of all particles in a given system. At every instant, the state of the system is uniquely represented by a single "phase point" in this space. Consequently, the phase space trajectory denotes the chronologically ordered set of "phase points" (generally $6 N$-tuples of numbers for a system of $N$ independent particles) during the evolution of the system in time. In formulae, we shall specify a "phase point" by the two vectors of all particle positions, $\mathbf{q}(t)$, and all particle momenta, $\mathbf{p}(t)$, at the time $t$.

Any observable, $o$, is generally defined as a function of the phase space trajectory. Depending on the definitions of the observables, they can be grouped into different classes [allen2003]. The presumably most frequently used class constitute the so-called "static observables". They are simply "time averages" over the time intervals, $\tau$, of the instantaneous values of the microscopic functions, $o(\mathbf{q}(t), \mathbf{p}(t))$, of the individual "phase points" along the phase space trajectory

$\mu(o)=\frac{1}{\tau} \int_{0}^{\tau} o(\mathbf{q}(t), \mathbf{p}(t)) \mathrm{d} t$

Note, the static observables, such as particle densities, temperatures, or pressures for instance, relate in an equilibrium system exclusively to its state and are therefore independent of the particle masses.

Another class of observables comprises the "root mean square (RMS) fluctuations" of the instantaneous function values, which underlie any static observable, about their "time averages"

$$
\sigma(o)=\sqrt{\frac{1}{\tau} \int_{0}^{\tau}(o(\mathbf{q}(t), \mathbf{p}(t))-\mu(o))^{2} d t} .
$$

Such root mean square fluctuations can serve as a crude means to assess the reliability of the "timeaverages" that they relate to [haile1997], [rapaport2004]. 
Finally, there are the so-called "dynamic observables". They are defined as extensions of the former two classes using the correlation function formalism, see e.g. [mcquarrie2000], and account for the time evolution of the system on the microscopic scale. Note, the dynamic observables, such as viscosities, heat conductivities, or diffusion for instance, relate in an equilibrium system exclusively to the processes and depend therefore on the particle masses.

In this work, we are mainly concerned with the observables from the first two classes introduced above. For the sake of simplicity, we shall henceforth denote the "static observables" as "observables" and the "RMS fluctuations" of the instantaneous function values underlying a "static observable" as its "standard deviation". In formulae, graphs, and tables the "static observables" will be designated with the letter " $\mu$ " and the related "standard deviation" with the letter " $\sigma$ " followed by the symbol of the instantaneous function values in brackets. This notation was adopted from [bendat2000] in the interest of improving readability.

In principle, the observables are computable over an arbitrarily long time interval, $\tau$, considering an arbitrary number, $N$, of particles, see equations (3-1) and (3-2) above. Macroscopic observables, as known e.g. from thermodynamics or hydrodynamics, are typically determined to describe the characteristic behaviour of a system. This requires eventually that the sample spans a "large enough" number of particles and a "sufficiently long" time interval, $\tau$, [callen1985]. It is not easy at all to establish what "large enough" and "sufficiently long" means; this may be quite different for different observables. It is also, however, only on these scales that the computed "observables" have their "macroscopic meaning".

There are nowadays experimental techniques that go well beyond the classical observables of phenomenological thermodynamics or hydrodynamics and probe matter in space-time frames down to the molecular scales, e.g. time resolved spectroscopies [lyons1978], [coppey1980], [beddard1993], [yoshizawa1999], [reid2003] or microscope techniques, like atomic force microscopy (AFM) [binnig1986], [carpick1997], [humphris2005], with temporal or spatial resolutions down to a few femtoseconds or nanometres. In keeping with these techniques it makes sense to determine from the simulations "observables" also over similarly short time intervals and small dimensions. Of course, the more $N$ and $\tau$ approach the molecular scales, the larger the fluctuations, i.e. the uncertainty, will be. 
Turning briefly to nonequilibrium processes: If the processes in a nonequilibrium system proceed moderately [degroot1960], [haase1963] in the sense of irreversible thermodynamics (see part 10), one can expect to compute in analogy with the above-mentioned techniques meaningful observables. Otherwise the spatial and chronological resolution necessary to catch the system behaviour may raise the uncertainties of so-computed values over all bounds. The scales down to which an observable can be determined with an acceptable uncertainty depend on the type of this observable. Note that the uncertainty can possibly even be disentangled in equilibrium or stationary nonequilibrium systems into a "finite-size uncertainty", due to the finiteness of the underlying system, and a "statistical uncertainty ", due to the incompleteness of the phase space trajectory [haile1997].

Any observable can in principle be determined by averaging the applicable microscopic function, $o(\mathbf{q}(t), \mathbf{p}(t))$, see equations (3-1) and (3-2). Some observables, so-called mechanical ones [hill1986], are a-priori easily defined in terms of such functions, $o$, e.g. the pressure, $P$, the internal energy, $E$, the volume, $V$, the momentum vector, $\mathbf{p}$, or the particle fluxes. The definitions of additional observables can be derived from the previous mechanical ones by appealing to the phenomenological theories like thermodynamics or hydrodynamics. In this way, e.g. expressions for the temperature, $T$, the entropy, $S$, the thermodynamic functions (Helmholtz free energy, $A$, Gibbs free energy, $G$, enthalpy, $H$ ) or the heat fluxes are obtained [mcquarrie2000]. Likewise, the statistical mechanics of transport phenomena was first derived in fundamental works by J.G. Kirkwood [kirkwood1946], [kirkwood1947], [kirkwood1949], [kirkwood1950]. However, many of these expressions are difficult to compute in practice, e.g. the entropy related properties.

One can usually recognise from the microscopic definition (the function $o$ ) of an observable down to which length and time scales its determination makes sense. We shall determine specifically the distributions of so-called "single-particle observables", such as the one-particle density, the momentum, or the kinetic energy, on the microscopic scale. Their functions are simply sums of the contributions from the individual particles [rowlinson2002]. 


\subsection{Simulations}

We mentioned in part $\mathbf{3}$ the characteristic assumptions of classical statistical mechanics which, in combination with further "modelling assumptions", constitute the foundation to translate a "physical system" into a "model system" of coupled equations of motion. They can be solved based on a suitably chosen initial phase point. MD simulations thus preserve, in keeping with Boltzmann's vision, the time evolution of the system, an aspect not often exploited in the traditional approaches of statistical mechanics (see part 9).

Since analytical solutions generally do not exist for multi-particle model systems, they must be solved numerically "by force". However, the limited available (or even technically feasible) computing power and storage capacity suffice to compute barely a sample, or section, of the full phase space trajectory. Moreover, the computation requires in practice further simplifications. Thus, the system is typically downsized to a manageable number of particles, of the order of a few hundreds to ten thousands. Moreover, a further array of "technical simplifications" is implemented, e.g. for the computation of the interactions through periodic (toroidal) boundary conditions together with the so-called "minimum distance convention" or the truncation of the interatomic forces at a certain "cut-off radius". These simplifications, explained thoroughly e.g. in [haile1997], [allen2003], are to be implemented in such a way that the "simulation system" (see section 3) still parallels the guiding "physical system" as closely as possible.

The computed section of the trajectory and thus also the determined observables are subject to errors. They can be crudely distinguished by their sources into "modelling errors" due to the modelling assumptions, "finite size errors" due to the stringent spatial limitation of the systems, "simulation errors" due to the technical simplifications, and "numerical errors" e.g. from the integration of the equations of motion (see section 3). Nevertheless, as long as the section remains representative, it can be analysed with respect to the observables of interest in the way described in section 3.1. One has to keep in mind these inaccuracies as well as the "natural" fluctuations of the system, explained already in the aforementioned section, when extrapolating from the "simulation system" to the guiding "physical system". 
It is a difficult task to assess or even ensure the representativeness of a MD simulation. The biggest problem is usually the incompleteness of the computed section of the phase space trajectory: A simulation can only count as representative with respect to certain events if they occur at approximately the same extent and frequency as along the full trajectory. While this condition cannot be strictly verified, the calculation of certain time-correlation functions [frenkel2002] offers some insight. In addition, comparing the several numerical values obtained for the observables from different parts of a computed trajectory can give hints [fincham1986]. Alternatively, the values obtained over different trajectories of the same system can be compared, each of them starting from an entirely different initial phase point. In the course of this work, we shall conduct such comparisons exemplarily for one of our simulation systems in section 4.4.3. Stability estimates of the computed observables with respect to the "simulation errors" and "numerical errors" can serve as a further criterion. Such estimates are often obtained through parameter variations.

Obtaining a suitable initial phase point that is consistent with the specification of the system state is also often not easy. Such points are usually constructed starting from more or less arbitrary initial conditions in "preparational simulations", which have, strictly speaking, no physical meaning. Thus, many "tricks of the trade" [allen2003] can be used in this phase of work in order to obtain a starting point as efficiently as possible. Chapter $\mathbf{4 . 4}$ will address this problem specifically for the systems to be simulated here. The trajectory computed during equilibration is of course discarded when the actual "production simulations" have been started.

A final remark concerning the noise in the observables: It can, in practice, hardly be disentangled into the various sources defined in this and in the previous section. The "statistical uncertainty" decreases generally for a given "simulation system" with increasing simulation length; "generally" because "modelling" and "simulation errors" of course increase with the number of numerical operations, i.e. with simulation length. When everything is said and done, one assumes that these errors are small and that the fluctuations observed in the system are the true ones associated with the "model system", and ultimately the guiding "physical system". 


\subsubsection{Equilibrium MD Simulations}

When the guiding "physical system" is in thermodynamic equilibrium, the derivation of the corresponding "simulation system" is often relatively easy; in particular, when it comes to the simple systems that are also considered in traditional statistical mechanics. Thus the interactions of such systems with their surroundings can be typically emulated by using periodic boundary conditions [allen2003]. Since they preserve the particle numbers $N_{\check{\iota}}$ of the different substances $\check{\iota}$, the system volume $V$, and the total system energy $E$, a so-specified $N_{i} V E$ system can be readily simulated if a corresponding initial phase point is provided. The equations of motion are usually adapted with additional pseudo-forces, e.g. to establish a constant temperature or pressure through interactions with a heat or a pressure bath $\left(N_{i} V T\right.$ or $N_{i} P T$ systems $)$. Also more complex boundary conditions can be implemented using such pseudo-forces, e.g. a solid wall. The reader is referred to specialised textbooks [haile1997], [sadus1999], [frenkel2002], [allen2003], [rapaport2004] for the details of these procedures. MD simulations of systems in thermodynamic equilibrium, abbr. EMD simulations, are well established, e.g. in the determination of all kinds material properties. A multitude of papers is published every year. See for example [beveridge1989], [benjamin1997], [heinz2005], [bopp2008] for more elaborate reviews. Not to be confused with equilibrium simulations, the preparational simulations, carried out to obtain a suitable initial phase point are, often called equilibration simulations. 


\subsubsection{Nonequilibrium MD Simulations}

Similar to the EMD simulations from the previous section, nonequilibrium molecular dynamics (NEMD) simulations attempt to compute a representative section of the phase space trajectory. We are, however, dealing with open or closed dissipative systems, now. It is a difficult task to implement the exchange of mass or energy with the surroundings. The necessary assumptions and simplifications can strongly bias the representativeness of the computed phase space trajectory. Even though simulations of nonstationary systems are still subject to research, see e.g. [rapaport1991], [hirshfeld1998], [hirshfeld2000], [bougie2002], [okumura2003], [okumura2004], [bougie2005], there is already a broad body of experience with simulations of stationary nonequilibrium systems [sarman1998], [sadus1999], [hafskjold2002a], [allen2003], [rapaport2004]. These are systems that have fully adjusted to their indefinitely maintained nonequilibrium boundary conditions and show no macroscopically observable change. Except for some "teething problems" to be discussed below, such stationary NEMD simulations often mimic surprisingly well the guiding "physical systems". Thus, reasonable values for the individual transport coefficients were determined in NEMD simulations by relating the associated driving forces with the observed fluxes in the simulation, e.g. in [evans1982], [tenenbaum1982], [evens1986], [bordat2002].

It soon emerged that complex experimental situations, e.g. involving several coupled transport processes or interfaces [hafskjold1993], [ikeshoji1994], [kjelstrup1996], [wold1999], [reith2000], [perronace2002], [xue2004], [galliero2005], [cieplak2006], [galliero2006], [koplik2006] could be reproduced only in so-called boundary driven (BD) NEMD simulations. In analogy with reality, these simulations incorporate the interactions of the system with its surroundings (see section 3 ) by introducing so-called "boundary regions" into the "simulation system". The equations of motion of those particles that sojourn in the "boundary regions" are perturbed so as to incorporate e.g. the inand outfluxes of energy or momentum as realistic as possible. New particles can be inserted or present ones removed in these regions to mimic the in- and outfluxes of mass.

The particles that sojourn outside of the boundary regions are subject to unperturbed Newtonian equations of motion, with forces resulting from the interactions with neighbouring particles only. During the simulation, this "intermediate region" is deflected into a nonequilibrium state, that is compatible with the conditions in the "boundary regions", by interaction, i.e. exchange of mass and 
energy, with them. In response to this deflection, the values of the observables of the "intermediate region" will generally differ from those obtained if that region were in thermodynamic equilibrium. For the differences to be clearly recognisable in BD NEMD simulations, however, very strong deflections of the "intermediate region" are necessary. Only then, its nonequilibrium behaviour emerges outside of the various simulation, statistical, and other errors inherent to the method.

The modifications of the equations of motion of the boundary particles vary depending on what one wants to achieve. A number of concepts have proven themselves, see e.g. [ashurst1975], [ciccotti1980], [trozzi1984], [hafskjold1995], [müllerplathe1997], [müllerplathe1999]. Mainly periodic boundaries or wall potentials are used to delimit the exterior of the "boundary regions". Straightforward insertion or removal of particles in the boundary regions is to be avoided, as far as possible, since it entails strong non-physical behaviour - particularly in dense matter. In- or outfluxes of energy or momentum are well accounted for by suitably adjusting the boundary particle positions and momenta. As an example: In a first stage, preliminary positions and momenta are computed by using the Newtonian equations of motion, with force terms only from the interactions of the particles with their neighbouring ones and with the boundaries. In a second stage, the individual particle momenta are manipulated according to some "allocation formula" so as to reproduce, on the average, the in- or outfluxes of energy or momentum.

Even when adhering to the aforementioned concepts, the various so far implemented perturbations of the "boundary regions" manage to approximate reality only with moderate success. Take, for instance, the occurrence of "temperature jumps" at "boundary regions" that establish heat fluxes in NEMD simulations [ciccotti1980], [hafskjold1993], [müllerplathe1997]. Such "teething problems" are disruptive but may not be overrated. Instead, a system may often exhibit some unphysical behaviour as long as it fades away quickly inside the "intermediate region". Then, the particles sufficiently distant from the boundaries can be assumed to behave as would "real" nonequilibrium matter (subject, of course, to the "modelling assumptions" and "technical simplifications"). It constitutes the actual object of investigation. Figure 11 summarises this situation. The behaviour of the nonequilibrium matter has been shown to agree with the predictions of "thermodynamics" and "hydrodynamics" if the deflection out of equilibrium is not too strong [rosjorde2000], [rosjorde2001], [hafskjold1995], [rapaport2006], [xu2006]. Nevertheless, the systems behave very differently and simulation results must always be carried over to the physical system with great care. 
Representative initial phase points for the actual production BD NEMD simulations are obtained in preliminary "steadying simulations" during which the "intermediate region" can adapt to its deflection away from equilibrium. The procedure corresponds to the one in the "equilibration simulations" prior to EMD production runs. 


\section{General Strategy of this Work}

It is virtually impossible to translate any of the common experimental setups used for exploring the thermocapillary convection (see chapter 2.1), entirely into a system ready for MD simulation. This is, however, not required for our purposes. We are interested in the thermocapillary effect in general. Hence, we devise our own simulation setup in such a way that the thermocapillary effect occurs realistically (somewhere) inside the system (see Figure 12). It is sufficient that the crucial processes involved in the thermocapillary effect become identifiable. See section $\mathbf{4 . 1}$ for further details.

Let us stress here again that the interpretation of the simulations must be done with caution due to the noise in consequence of the limited systems size and of the unavoidable assumptions and simplifications required by the method. Before we can proceed with the actual investigations of the thermocapillary effect, we must therefore gain certainty to which extent our simulations parallel reality. We shall largely proceed by analogy between experiment and simulation. Within this procedure we will deduce in section 4.2 the distributions of the local observables to be expected in our simulation systems. If we can find such distributions in our simulations, we shall conclude that they are physically sound and sufficiently close to reality. In keeping with this line of argumentation we shall check the distributions of the local observables in our first simulations of the interfacial system. Chapter $\mathbf{5 . 1}$ will show that these distributions, in particular the convection patterns, appear exactly as expected, except for some minor anomalies in terms of the temperature jumps at the boundary regions.

Computing power and memory capacity are the limiting factors of our MD simulations. In section 4.3, we shall explain how we meet these factors in the execution and analysis of the simulations. In particular, details on the definitions and the computation of our observables will be given. Since it fits best in this context, we shall also introduce the scheme which we will apply to summarise the results of the individual simulations for better understandability and comparability. Section 4.4 will go into the preparation and production simulations of our systems. Finally, Section $\mathbf{4 . 5}$ will describe the simulation code and hardware used. 


\subsection{Setup of the "Simulation Systems"}

The existence of a lateral temperature difference at a solid-fluid or fluid-fluid interface is the prerequisite for the occurrence of the thermocapillary effect (see part 2). Reproducing in a "simulation system" such an arrangement is difficult. After all, we cannot be externally enforce it in a straightforward way. Instead, the individual design aspects of a system must be chosen from countless variants and mutually matched in such a way that the intended arrangement indeed arises out of a simulation (see Figure 12). Thus, an interface in thermodynamic equilibrium, for instance, orients depending on the system dimensions and boundary conditions as well as the thermodynamic state of the given mixture (see section 4.1.1). In addition to the requirements by the intended arrangement, the various ones required by the method must be met, namely simulatability, approximation of reality, and simplicity. They even partly contradict themselves. In solving this design problem we draw on the experiences from previous works as much as possible. Thus, the principle of minimum entropy production [degroot1960], [prigogine1961], [degroot1969], [jaynes1980] enables predictions of what the favourable arrangement in a stationary nonequilibrium system looks like. Moreover, we build on a widespread simulation code which implements the common assumptions and simplifications of MD simulation in a generally accepted way. Upgrades to the code are only made as far as necessary for our purposes. See section $\mathbf{4 . 5}$ for more details.

Earlier simulations of partially miscible substances provided us a basis for the setup of our "simulation system". Section 4.1.1 will briefly review the pertinent literature concerning the general setup and behaviour of such systems simulated. As will be described, the simplest ones involve a partially miscible binary mixture, confined to a rectangular parallelepiped arrangement by periodic boundaries. When this mixture is made subject to the appropriate thermodynamic conditions, it tends to separate into two continuous phases parallel to the smallest "boundary surfaces" (see Figure 13). Note, we see such a separation in our equilibration simulations. Section 4.4.1 will describe them in more detail. Provided the same behaviour holds also in the nonequilibrium states required by this work, the setup can be reused to a large extent. Different temperatures $\left(T_{C}\right.$ and $\left.T_{H}\right)$ must now only be established along the interface in NEMD simulations to allow for the thermocapillary effect to occur. Hence, we positioned two thin rectangular parallelepiped "boundary regions" perpendicular to the interfaces into an "interfacial system" of the kind previously described (see Figure 14): one of the "boundary regions" in the middle and the other one symmetrically at the 
"boundaries" of the periodic system. Its symmetry is thus preserved leading to four "identical" copies of the intended arrangement. Note that the term "boundary regions" does not apply literally due to the periodicity of our system. Rather, they can also be seen as located inside the "simulation system", detached from its boundaries. Consequently, we shall henceforth name the "boundary regions" as "thermostated regions" since this denotation is more appropriate. The thermostating itself was implemented by an algorithm that alters the kinetic energies of the particles inside the "thermostated regions" at each time step in a suitable way to establish the desired temperatures therein. Section 4.1.4 will give further details on the exact locations of these regions and the various "thermostats" applied to them.

We positioned a Cartesian coordinate system in the middle of our "nonequilibrium interfacial system". The coordinate axes (also shown in Figure 14) are oriented according to the following convention: The $z$-axis lies perpendicular to the interfaces and the $y$-axis perpendicular to the "thermostated regions". Consequently, the $x$-axis runs along the direction in which the system is homogeneous. Moreover, we defined that the colder target temperature, $T_{C}$, is always established in the "thermostated region" with $y$-coordinates around zero. The hotter target temperatures, $T_{H}$, are thus always established in the "thermostated region" at the outermost $y$-coordinates.

As for the substances, we confined ourselves a priori to so-called non-charged "one-centre LennardJones (LJ) particles", as they are frequently used in MD simulations. Such model particles consider the essential properties of (non-polar) substances in the simplest-possible way through pairwise additive interaction potentials. They contain a repulsive part, which represents, loosely speaking, the volume of the particle, and an attractive part, which represents the typical intermolecular interaction between non-charged molecules. The "molecular models", or "force fields", describing mixtures of different species of such particles possess only few parameters and are thus relatively simple. They are the so-called LJ parameters for the pairwise interactions between like and unlike particles, a technical parameter called the "cut-off radius" $r_{c}$, and the particle masses. In particular, the $\mathrm{LJ}$ parameters must be in within certain ratios to each other for a mixture of different particles to exhibit partial miscibility. We ensured such ratios by using the Lorentz-Berthelot mixing rule [maitland1981], [delhomelle2000]. It specifies, when given the LJ parameters for the interactions of like particles, implicitly those for the interactions of unlike particles through so-called mixing parameters, $\xi$ and $\eta$. We shall neglect one of them, $\eta$, by setting it to unity. Section 4.1.2 will give further details on this mixing rule and on the numerical values of our "particle parameters". Since both substances contained in our "simulation system" usually copy Argon, we shall call them ArA 
and ArB in general. The particle masses affect the static observables only in our NEMD simulations, as we shall see, for instance, in section 5.2.2.

The above design of our simulation system is complete except for the numerical specification of the "system constraints", i.e. the system dimensions $\left(L_{x}, L_{y}, L_{z}\right)$, the target temperatures of the "thermostated regions" ( $T_{C}$ und $\left.T_{H}\right)$, and the particle numbers of both species $\left(N_{A r A}\right.$ and $\left.N_{A r B}\right)$. Only if their values are compatible with the ones of the particle parameters, the mixtures actually separate into the two liquid phases, as intended. In fact, several entities of our system, each with different numerical values of the "system parameters", i.e. "particle parameters" and/or "system constraints", were be simulated in the course of this work to study their influence on the system behaviour. For the sake of clarity, we shall designate these simulations using a special notation. It consists of the letter "N" for nonequilibrium followed by the names of the substances, the numerical value of their mixing parameter, $\xi$, the cut-off radius, $r_{c}$, the numerical values of the "system constraints", see above:

$\mathrm{N}-\mathrm{ArA}-\mathrm{ArB}-\xi-r_{c}-N_{A r A}-N_{A r B}-L_{x} \times L_{y} \times L_{z}-T_{C^{-}} T_{H}$.

In this notation, all distances are given in nanometres $\left(1 \mathrm{~nm}=1 \cdot 10^{-9} \mathrm{~m}\right)$ and the temperatures in Kelvin (K). Our first "nonequilibrium interfacial system" - it contains an Ar1Ar2 mixture under the default constraints - is for example designated as:

N-Ar1Ar2-0.6-1.0-3346-3524-4.74x8.00x9.40-100-140 .

Later, we shall also simulate systems where the parameter values of interactions among $\operatorname{ArA}$ and among ArB particles represent the same substance. Then, its name appears two times in the designation, to identify the special character of the simulation, as e.g. in

N-Ar5Ar5-0.6-1.0-3346-3524-4.74x8.00x9.40-100-140.

We shall call such liquid-liquid systems "homophasic" to distinguish them from the "heterophasic" ones mentioned before.

All mixtures were also simulated for comparison in "corresponding equilibrium interfacial systems" as part of our research. They have either $N_{A r A}-N_{A r B}-L_{x} \mathrm{x} L_{y} \mathrm{x} L_{z}-T$ or $N_{A r A}-N_{A r B}-L_{x} \mathrm{x} L_{y} \mathrm{x} L_{z}-E$ constraints, where the letters " $T "$ and " $E "$ represent the global system temperature or energy. We shall use a slightly modified notation for these systems, which replaces the letter "N" by the letter "E". Other than that, the numerical values of the applicable system constraints are given as before after the length of the cut-off radius. In doing so, the global temperature is in Kelvin $(\mathrm{K})$ and the global 
energy in Attojoules $\left(1 \mathrm{aJ}=1 \cdot 10^{-18} \mathrm{~J}\right)$. The criterion for "correspondence" between equilibrium and nonequilibrium systems is that they have the same externally constrained system volume $V$ and the same particle numbers $N_{i}$. Moreover, the system temperature in the equilibrium system must coincide with the arithmetic average of the externally constrained boundary temperatures in the nonequilibrium system. In the EMD simulations with constrained system temperature the latter is essentially maintained through a Berendsen thermostat [berendsen1984] - "essentially" because we implemented the Berendsen thermostat using our "thermostating algorithm" for simplicity's sake. Go to section 4.1.4 for more details. Since a Berendsen thermostat can disturb the system behaviour [lemak1994], [leyssale2008], we conducted, for comparison, additional EMD simulations of the first system under the same constraints using a Nosé-Hoover thermostat [nose1984], [hoover1985] or under corresponding NVE constraints. In section 4.4.1, we shall find that there are no significant deviations between the results of the different simulations.

Finally, the phases observed in the equilibrium interfacial system were also reproduced separately in "corresponding nonequilibrium one-phase systems" for comparison. They have the same dimensions and temperature constraints as the corresponding nonequilibrium interfacial system but different particle numbers. We obtained them simply by multiplying the phase densities in the corresponding equilibrium interfacial system with the system volume. The notation introduced above shall also be used for the nonequilibrium one-phase systems. They can be recognised by the strongly deviating particle numbers, $N_{A r A}$ and $N_{A r B}$, of each species. 


\subsubsection{General Setup of the Liquid-Liquid Interfacial Systems}

Liquid-liquid interfacial systems at equilibrium have been studied several times in MD simulations, see e.g. [meyer1988], [toxvaerd1992], [buhn2004a], [geysermans2005]. These systems usually have a rectangular parallelepiped volume with the dimensions $L_{x}, L_{y}$ and $L_{z}$ in $x$-, $y$-, and $z$-direction. The partially miscible mixture to be studied is represented by $N_{\check{\imath}}$ particles of each constituent substance, $\check{\imath}$. According to common practice the systems are bordered by periodic boundaries. In this way, unwanted side effects can be avoided, as they would e.g. occur at a solid confinement. External fields, such as gravity, are omitted for the sake of simplicity. The thermodynamic state of the mixture is determined indirectly through the "specific boundary conditions" and the "initial phase point" of the simulations, e.g. in $N V T$ simulations by specification of the particle numbers $N_{\check{\imath}}$ of the individual substances $\check{l}$ in the initial system, the system volume $V$ through the system dimensions $L_{x}$, $L_{y}$, and $L_{z}$, and the system temperature $T$.

Depending on the chosen numerical values of the specific boundary conditions, an arrangement of two continuous liquid phases with a common interface establishes during equilibration, as can also be seen in section 4.4.1. In the establishment, the phases always arrange themselves in such a way with respect to each other that their interfacial area is minimal [scott1994], i.e. that the internal energy of the system becomes minimal [degroot1960], [prigogine1961], [haase1963], [degroot1969]. Within limits, this tendency can be taken advantage of, e.g. to favour a certain arrangement of the liquid phases by a suitable choice of the composition of the mixture. Thus, typically comparable volume fractions of both substances in binary mixtures are chosen to obtain a flat interface which is easier to analyse. More precisely, two flat interfaces occur parallel to the smallest surfaces of the rectangular parallelepiped system in consequence of the periodic boundaries. 


\subsubsection{Molecular Models and Force Fields}

In the "simulation system", all atoms (particles) of both substances, generally called ArA and ArB in this work (see section 4.1), are each represented by points carrying masses depending on the type of the particle, i.e. which species it belongs to. We used in most of our simulations the molecular mass of Argon $\left(m_{A r}=39.95 \mathrm{amu}=6.63 \cdot 10^{-26} \mathrm{~kg}\right)$, as found in the literature [atkins2002], for each particle type, $m_{A r A}$ and $m_{A r B}$.

The interparticle interactions, as they can be obtained from quantum mechanical calculations, see [mcquarrie1997] for a reference, were computationally too expensive for our simulations. Instead, we accounted for the interactions in the extremely simplified form of "effective" Lennard-Jones (LJ) pair potentials [stone2002], $u_{i j}$ :

$u_{i j}\left(r_{i j}\right)=4 \varepsilon\left[\left(\frac{\sigma_{i j}}{r_{i j}}\right)^{12}-\left(\frac{\sigma_{i j}}{r_{i j}}\right)^{6}\right]$.

Here, $\mathbf{r}_{i j}=\mathbf{r}_{j}-\mathbf{r}_{i}$ stands for the connecting vector between the particles $i$ and $j$. It has the length $r_{i j} . \varepsilon_{i j}$ and $\sigma_{i j}$ are the $\mathrm{LJ}$ interaction parameters that differ depending on the types of the interacting particles. If we add all pair potentials between the particles $i$ and $j$ in a system we obtain its potential energy $U$ :

$U=\sum_{i>j} u_{i j}\left(r_{i j}\right)$.

Its negative partial derivatives with respect to the locations of the different particles, $i$, produces the forces, $\mathbf{t}_{i}$, acting on them:

$\mathbf{\mathbf { }}_{i}=-\frac{\partial U}{\partial \mathbf{r}_{i}}=\left.\left.\sum_{j} \frac{\partial u_{i j}\left(r_{i j}\right)}{\partial \mathbf{r}_{i j}}\right|_{\mathbf{r}_{j}} \frac{\partial \mathbf{r}_{i j}}{\partial r_{i j}}\right|_{\mathbf{r}_{j}}$.

Six LJ parameters are necessary to describe all possible combinations of interacting particle types in our ArA-ArB mixture: $\varepsilon_{A r A-A r B}$ and $\sigma_{A r A-A r B}, \varepsilon_{A r A-A r B}$ and $\sigma_{A r A-A r B}, \varepsilon_{A r B-A r B}$ and $\sigma_{A r B-A r B}$. Many value sets of such LJ parameters have been developed empirically, especially in chemical engineering, to be able to reproduce specific experimental observations as closely as possible in MD simulations. We are, however, interested in the thermocapillary effect in a general way here. Accordingly, the simulation results are not to be compared with a particular chemical system, as noted already in part 
4. Thus, we simply chose characteristic LJ parameter values in the range of those typically used in the literature. They and the particle masses were moreover systematically varied to study their influence on the system behaviour. For the sake of clarity, we shall count all particles of the same mass and mutual LJ interaction parameters among the same substance, whatever the parameter values of the interactions with particles of other species may be. Different particle parameter values of Argon were taken from the literature [michels1949], [hansen1969], [kofke1993], [buhn2004a], [buhn2004b]. Sometimes, we even went so far as to give both components the same values for convenience. Such a choice still allows for the necessary partial miscibility of the mixtures. It rather depends on the subsequently discussed interaction parameters of unlike particles. To express the relationship of the simulated substances with Argon, we shall designate them simply in the order of appearance as "Ar1", "Ar2", etc. . In doing so, they count only once, even if they constitute both components of the mixture, such as in the Ar5Ar5 one studied in chapters $\mathbf{5 . 4}$ and 5.5, for instance. Nevertheless, it sometimes made sense to determine observables that relate to the ArA or the ArB component only, e.g. in verifying the similarity of both phases within the same system (see chapter 5.3). Then, the observables related to the first component have its designation followed by the encircled number with a white background, e.g. $\rho_{\operatorname{Ar}(5)}$. We append the encircled number with a black background instead, if an observable relates to the second component, e.g. $\rho_{\operatorname{Ar} \boldsymbol{F}}$. Table 3 lists the particle parameter values of all simulated substances together with their critical and triple point data. They were estimated from Kofke's [kofke1993] and Agrawal's [agrawal1995] dimensionless phase diagram for unary systems of one-centre LJ particles, similar to ours.

The spherical LJ-interaction is indeed very much simplified compared to the real interaction between two molecules as it can e.g. be obtained from quantum mechanical calculations, see e.g. [mcquarrie1997]. Many sets of such LJ-parameters have nevertheless been developed empirically, especially in chemical engineering, to reproduce observables (mostly thermodynamic) in MD simulations. We are here interested in the thermocapillary effect in a general way, not in a particular chemical system. Accordingly, the simulation results are not to be compared with such a system, as noted already in part $\mathbf{4}$. We thus chose for the interaction parameters some characteristic values, of the orders of magnitude typically used in the literature. Different Argon-like LJ parameter values for the interactions between like particles were taken from previous works by Buhn et al. [buhn2004a], [buhn2004b] or Hansen et al. [hansen1969]. Reasonable values for the LJ interactions between unlike particles, $\varepsilon_{A r A-A r B}$ and $\sigma_{A r A-A r B}$, were estimated from those for like particles by using a generalised version of the empirical Lorentz-Berthelot mixing rules [maitland1981], [delhomelle2000] 


$$
\begin{aligned}
\varepsilon_{A r A-A r B} & =\xi \sqrt{\varepsilon_{A r A-A r A} \varepsilon_{A r B-A r B}} \\
\sigma_{A r A-A r B} & =\frac{\eta}{2}\left(\sigma_{A r A-A r A}+\sigma_{A r B-A r B}\right) .
\end{aligned}
$$

In doing so, we set the so-called mixing parameter $\eta$ to its common value unity. The other mixing parameter, $\xi$, was reduced by a few tenths below its common value, also unity, to achieve thin interfaces and a wide miscibility gap between ArA and ArB. As a result, the setup could be somewhat downsized and thus computer time saved without loosing the generality of the simulations. Note, we used typically a relatively small mixing parameter, $\xi=0.6$, which is even lower than the one, $\xi=0.8$, in the previously mentioned works by Buhn et al. [buhn2004a], [buhn2004b].

In order to further reduce the computational costs of the force calculations, we cut all LJ pair potentials beyond a certain distance to zero. Thus, only the interactions between particles that lie closer together than this distance had to be computed. The introduction of such a cut-off radius, $r_{c}$, (as it is commonly called) for interactions that decrease with a sufficiently high power of the particle distance, $r_{i j}$, is a well understood technical simplification in MD simulations [allen2003]. The proper magnitude of the cut-off radius varies with the "simulation system" and the observables of interest [haberlandt1995]. It should be chosen as short as possible to reduce computational expense and as large as necessary to avoid artefacts. We therefore chose first a cut-off radius of $r_{c}=1 \mathrm{~nm}$, which slightly exceeds the often suggested length of 2.5 times the smallest LJ parameter $\sigma$ in the "simulation system" [haile1992]. Nevertheless, we additionally varied the cut-off radius later on (see section 5.2.1) to check its influence on the simulation results and thus ascertain our initial choice.

In the same spirit, several other such particle parameters were varied in our simulations. See Tables 3-4 for the particle parameters of the various species and mixtures simulated. 


\subsubsection{System Constraints}

The constraints of our nonequilibrium interfacial system, namely its dimensions $\left(L_{x}, L_{y}, L_{z}\right)$, the particle numbers $\left(N_{A r A}\right.$ und $\left.N_{A r B}\right)$, and the target temperatures $\left(T_{C}\right.$ und $\left.T_{H}\right)$ of the thermostated regions, were subject to diverse and partially even contradictory requirements. Let us first recompile them here again from the previous sections.

A simulation system must be generally as large as necessary to reduce finite size effects but as small as possible to save computational expense (see section 3.2). Note, when talking about the "size" of a system here, we think of its dimensions and its particle numbers. This rather general requirement means specifically a flat simulation cell for our purposes. Its $y$-and $z$-dimensions, $L_{y}$ and $L_{z}$, must be clearly larger than customary in MD simulations to reduce the interference between both interfaces. Moreover, the unwanted system behaviour due to the thermostating can abate in this way to acceptable levels between the "thermostated regions". We concurrently must make the remaining $x$-dimension, $L_{x}$, and thus the $x y$-cross sectional area, relatively small so that the interfaces, if they occur, arrange preferably lateral to the temperature gradient. See chapter 4.1.

There is, however, even more necessary than just choosing the correct system dimensions. They must also be consistent with the thermostated temperatures. Only then, the mixture assumes a partially miscible liquid state throughout the entire system. Even if such a state was given, their difference still has a special importance. It must be large enough that the characteristic nonequilibrium behaviour stands clearly out from the noise inherent to the simulation and concurrently small enough that the system behaviour does not become nonstationary and thus impossible to analyse. We are, therefore, forced to apply temperature gradients of the order of $10 \mathrm{~K} / \mathrm{nm}$, as customary in BD NEMD simulations. Whatever the response of our nonequilibrium systems, particularly with the expected thermocapillary convection in mind, is, it must be seen if their behaviour remains physical and stationary under such exorbitant gradients.

We went back to previous works by Buhn et al. [buhn2004a], [buhn2004b] in order to break up the above ring of interdependent requirements. The authors studied interfacial systems of partially miscible liquids, very similar to our Ar1Ar2 mixture, in EMD simulations. There was a focus on 
system states between the corresponding temperature and average density pairs of ( $T=100 \mathrm{~K}$, $\left.\rho=20.62 \mathrm{~nm}^{-3}\right)$ and $\left(T=138 \mathrm{~K}, \rho=18.16 \mathrm{~nm}^{-3}\right)$. Using these simulations as a guidance (see also section 4.4.3), we chose the following default values for the external constraints of our nonequilibrium interfacial system: It has the dimensions $L_{x} \times L_{y} \times L_{z}=4.74 \times 8.00 \times 9.40 \mathrm{~nm}$. The numbers of particles are $N_{A r A}=3346$ und $N_{A r B}=3524$, which is of the same order of magnitude as required above. In total this makes $N=6780$ particles at an average system density of

$$
\rho=\frac{6870}{4.74 \mathrm{~nm} \cdot 8.00 \mathrm{~nm} \cdot 9.40 \mathrm{~nm}}=19.27 \mathrm{~nm}^{-3}
$$

The target temperatures of the "thermostated regions" are $T_{C}=100 \mathrm{~K}$ und $T_{H}=140 \mathrm{~K}$. While the system density is medium-sized compared to the one in the aforementioned studies by Buhn et al., the target temperatures have approximately the extreme temperature values simulated by them. Thus, partial liquid miscibility should likely occur also in our simulations, in particular if we keep the further reduced mixing parameter, $\xi$, in mind. Nevertheless, we double-checked again in the first simulations if the systems behave as intended. The results, they will be described in chapter 5.1, did not suggest otherwise. Only in few of our simulations use different constrained values from the ones from above. If they were varied, then only to study their influence on the system behaviour.

Out of curiosity, we ranked the states of our mixtures additionally in comparison to those of the pure substances under the same default constraints. Figure 15 indicates the range of temperatures over the average density. Both were reduced to dimensionless numbers by the LJ parameters of the different pure substances. We can clearly see how some of these dimensionless ranges extend below the bubble point line or even the triple line. Their data were taken from the dimensionless phase diagram of pure Ar-like particles by Agrawal [agrawal1995] et al. and Kofke et al. [kofke1993]. Apparently, the pure substances tend to evaporate and melt only at higher temperatures than the mixtures, since they do not exhibit such phase changes in our simulations. Part $\mathbf{1 1}$ addresses the phase behaviour of the mixtures in more detail. 


\subsubsection{Thermostating Algorithm}

In BD NEMD simulations, the momenta of the particles in the "boundary regions" are typically manipulated after each "integration step", as described in section 3.2.2, to carry out the exchange of heat with the "surroundings". Such a manipulation requires above all a realistic formula to allocate the in- or outflux of heat in terms of kinetic energy on the individual particles. Concurrently, the linear momentum of the simulated system must be preserved in the allocation. Different "allocation formulae" fulfilling these requirements have proven themselves according to the literature [tenenbaum1982], [müllerplathe1997]. We picked up a formula by Hafskjold et al. [hafskjold1993] which preserves the overall linear momentum of each affected "boundary region" separately and entails relatively moderate non-physical behaviour in the "intermediate regions". The momentum preservation has the advantage that the "boundary regions" cannot shear the "intermediate region" and thus interfere with the expected "thermocapillary convection".

In the application of the chosen allocation formula, we assumed that the equipartition principle from equilibrium statistical mechanics [mcquarrie2003] is fulfilled. Although this assumption is certainly wrong in our NEMD simulations, we adhered to it for lack of better alternatives. In order to comply with this principle, different amounts of kinetic energy must be allocatable on the $x$-, $y$-, and $z$ movement of the particles in a "boundary region". We shall explain exemplarily further below how we implemented the allocation of a given amount of kinetic energy, $\Delta K_{x}$, on the particle movement in $x$-direction. A similar procedure is applied to the movement in the other directions.

The "allocation formula" does, however, not specify according to which criterion, e.g. to establish constant heat flows, or constant temperatures, or ...), kinetic energy should be introduced into or removed from the "boundary regions". Hafskjold et al. originally applied their "allocation formula" as part of a heat exchange (HEX) algorithm [hafskjold1993]. This algorithm enforces an externally predetermined stationary heat flux by withdrawing the corresponding amount of kinetic energy from the particles in a so-called cold "boundary region" and adding the same amount to the particles in another so-called hot "boundary region" after each "integration step". 
It is however more intuitive for our purposes to apply thermostats that maintain each "boundary region", henceforward called "thermostated region", at a temperature of choice. These thermostats require, however, an additional control mechanism. Its purpose is to specify the amounts of kinetic energy that must be coupled into or out of a thermostated region to establish the desired

temperature, $\hat{T}$, therein. In order to comply with the abovementioned equipartition principle, this mechanism must consist formally of three mutually independent entities, so to speak. Each entity is occupied with the particle movement in another direction. Using again the particle $x$-movement as an example, we shall explain further below how the related amount of kinetic energy, $\Delta K_{x}$, was computed. This computation is conducted likewise for all directions in each thermostated region after every "integration step" of the Newtonian particle equations of motion. The thus obtained amounts of kinetic energies, $\Delta K_{x}, \Delta K_{y}$, and $\Delta K_{z}$, were then allocated on the particles in the each region using the allocation formula above. Only after the allocation, we considered the phase points in the analysis of the system behaviour.

\section{Allocation Formula}

In the allocation of $\Delta K_{x}$ on the individual particles, $i$, in a "thermostated region" their $x$-velocities, $u_{x, i}$, are scaled to, $\hat{u}_{x, i}$, by the same factor $\left(1+\alpha_{x}\right)$ and shifted by $\beta_{x}$ to preserve concurrently the total $x$-momentum of the particles:

$\hat{u}_{x, i}=\left(1+\alpha_{x}\right) u_{x, i}+\beta_{x}$ with $\beta_{x}=-\alpha_{x} \frac{\sum_{i} m_{i} u_{x, i}}{\sum_{i} m_{i}}=-\alpha_{x} u_{x, c o m}$.

The parameter $\alpha_{x}$ can be computed from the total kinetic energies of the particle movement in $x$ direction before, $K_{x}$, and after, $\hat{K}_{x}$, the allocation:

$$
\Delta K_{x}=K_{x}-\hat{K}_{x}=\frac{1}{2} \sum_{i}\left[m_{i}\left(\left(1+\alpha_{x}\right) u_{x, i}-\alpha_{x} u_{x, c o m}\right)^{2}-m_{i}\left(u_{x, i}\right)^{2}\right] .
$$

In order to do so, this equation can be rearranged to the quadratic form

$0=\alpha_{x}^{2} a_{x}+\alpha_{x} b_{x}+c_{x}$

with the coefficients

$$
\begin{aligned}
& a_{x}=\sum_{i} m_{i}\left(u_{x, i}-u_{x, \mathrm{com}}\right)^{2} \\
& b_{x}=\sum_{i} 2 m_{i}\left(u_{x, i}^{2}-u_{x, i} u_{x, \mathrm{com}}\right)=2 a_{x}
\end{aligned}
$$


$c_{x}=\sum_{i} m_{i}\left(u_{x, i}^{2}-\hat{u}_{x, i}^{2}\right)=-2 \Delta K_{x}$.

We define $\alpha_{x}$ to be

$\alpha_{x}=-1+\sqrt{1-\frac{c_{x}}{a_{x}}}$

from the two possible solutions of the quadratic equation because the other solution would reverse the particle movement.

Note that two important restrictions must be kept in mind when applying our "allocation formula" above. Thus, $a_{x}$ amounts to twice the kinetic energy of particle movement relative to the centre of mass (com) movement in $x$-direction and can therefore only be equal or larger than zero, $a_{x} \geq 0$. Consequently, $\alpha_{x}$ becomes imaginary if $c_{x}$ is larger than $a_{x}, c_{x}>a_{x}$, i.e. more kinetic energy than $a_{x} / 2$ is to be withdrawn by the thermostat. Moreover, each "thermostated region" must, at all times, contain at least two particles and they may not be moving at the same velocity in a certain direction. In practice, none of the above restrictions was violated during any of our production simulations.

\section{Temperature Control Mechanism}

We implemented a general temperature control mechanism that resembles a PID controller from control systems [unbehauen2002]. Thus, for instance, the total amount of kinetic energy, $\Delta K_{x}$, to allocate on the $x$-movement of the particles in a thermostated region is computed through the equation:

$$
\Delta K_{x}=k_{P}\left(\hat{K}_{x}-K_{x}\right)+k_{I} \sum_{t=0}^{n}\left(\hat{K}_{x}-K_{x}(t)\right)+k_{D}\left[\left(\hat{K}_{x}-K_{x}\right)(t)-\left(\hat{K}_{x}-K_{x}\right)(t-1)\right] .
$$

Here, $K_{x}$ is the prevailing total kinetic energy of the particles, $i$, in a "thermostated region" as computed from their masses, $m_{i}$, and their velocity components in $x$-direction, $u_{x, i}$, right after an "integration step":

$$
K_{x}=\sum_{i=1}^{N} \frac{1}{2} m_{i} u_{x, i}^{2}
$$

The corresponding desired total kinetic energy, $\hat{K}_{x}$, is obtained from the total particle number, $N$, and the desired temperature, $\hat{T}$, through the formula, 
$\hat{K}_{x}=\frac{1}{2} N k \hat{T}$

based on the assumption from the beginning of this section that the equipartition principle holds. The difference between the desired and the prevailing total kinetic energy, $\left(\hat{K}_{x}-K_{x}\right)$, contributes through three additive terms to $\Delta K_{x}$. The first summand acts as a "proportional term" and the second one as an "integral term" whose sum runs over all time steps from the first one to the respectively latest one of a simulation. Finally, we implemented a "derivative term" which considers the change of the difference, $\left(\hat{K}_{x}-K_{x}\right)$, from the latest time step to its preceding one. The terms are weighed by the parameters $k_{P}, k_{I}$, or $k_{D}$ that had to be tuned so as to ensure an opportune functioning of the thermostats. In the tuning we defined, for simplicity's sake, that each parameter has in every thermostated region the same value for all three directions of movement. Moreover, we omitted the "derivative term" (by setting $k_{D}$ to zero) after a few trial simulations because the fluctuations in the difference, $\left(\hat{K}_{x}-K_{x}\right)$, were too strong. The results of the trial simulations are not reported here for the sake of brevity.

We rather concentrated on finding suitable values for the parameters $k_{P}$ and $k_{I}$. In fact several thermostats with different parameter values were applied in the course of this work. In our first NEMD simulations, we set $k_{P}$ to one, $k_{P}=1$, and $k_{I}$ to zero, $k_{I}=0$. Such thermostats, henceforward called "strict", establish the desired temperatures exactly after every "integration step" but strongly bias the observable heat flux, as we shall see in chapter 5.2.

We therefore migrated to other variants with values of $k_{P}$ between zero and one, $0<k_{P}<1$. These variants adjust the kinetic energy of the thermostated regions more gently; a procedure which has been proposed by Berendsen et al. [berendsen1984] for NVT simulations. It works quite satisfactorily in them [allen2003]. We used such "Berendsen thermostats" for most of our EMD simulations in this work. In doing so, our allocation formula, which basically differs from the one originally used by Berendsen et al. only in terms of the additional shift of the particle velocities, is retained for simplicity's sake. As recommended by the authors, we set the proportional term to $k_{P}=\delta t / t_{T}=0.0125$, where $t_{T}=0.4 \mathrm{ps}$ is a time constant and $\delta t=5 \mathrm{fs}$ the length of our time steps.

A proportional term between zero and one, $0<k_{p}<1$, does, however, not suffice to establish the desired temperatures in our NEMD simulations. The failure results from the average net exchange of heat between the thermostated and their "intermediate regions". We therefore considered 
additionally the integral contribution in the computation of $\Delta K_{x}$ to compensate for the temperature deviation. The thus obtained thermostats shall be called "loose" as opposed to the strict ones above. We shall explain in section 5.2.3 how we determined in several trial simulations the parameter values of $k_{P}=0.001$ and of $k_{I}=0.0001$ that we used eventually for the loose thermostats.

We managed to use the same program code for EMD and NEMD simulations by reading the parameters $k_{P}, k_{I}$, and $k_{D}$ as well as the locations of the thermostated regions from the input files. These locations are specified by two parameters, $y_{C}$ and $y_{H}$. Particles with an absolute $y$-coordinate smaller than $y_{C}$ sojourn in the cold and ones with an absolute $y$-coordinate larger than $y_{H}$ sojourn in the hot thermostated region. In the EMD simulations, we set $k_{P}=0.0125, k_{I}=k_{D}=0$ and made the cold region comprise the entire system while the hot region was located somewhere outside. The exact values of $y_{C}$ and $y_{H}$ are not important as long as $y_{H}>y_{C}$ and $y_{C} \geq L_{y} / 2$, with $L_{y}$ being the $y$-dimension of the simulation system. In the NEMD simulations, on the other hand, we set $y_{C}=0.125 \mathrm{~nm}$ and $y_{H}=L_{y} / 2-y_{C}$, which makes each thermostated region $0.25 \mathrm{~nm}$ thick. Depending on the variant to use, we specify either $k_{P}=1$ and $k_{I}=k_{D}=0$ for the "strict" or $k_{P}=0.001, k_{I}=0.0001$, and $k_{D}=0$ for the "loose thermostats". When applying the "loose thermostats", we simply disregard in the analysis that the "integral term" must first grow over the approximately first thousand time steps (5ps) of a NEMD simulation in order to compensate for the average net exchange of heat between the thermostated and their intermediate regions. 


\subsection{Expected Behaviour of our "Simulation Systems"}

As described in section 4, we intend to study in this work the general features of the thermocapillary effect and designed suitable simulation systems for this purpose. Before we came to the actual studies, we wanted to make sure that we knew which behaviour to expect in the simulated systems. Direct quantitative comparisons with experimental situations would be one way to proceed. This procedure is, however, impossible, simply because of the small system sizes or, where applicable, the extreme temperature gradients required by the method. Instead we settled for a sort of "thought experiment" in which we imagined the behaviour to expect of the systems exhibiting the thermocapillary effect, of course in analogy with experiences from physical experiments or earlier MD simulations. These experiences are extensive enough to delimit unphysical system behaviours and to even predict the qualitative distributions of the local thermodynamic and hydrodynamic observables. If we find that the predictions are actually met in our simulations (as we will in part 5), we shall conclude that their behaviour is sufficiently close to reality and derive insights from them.

Before we begin our thought experiment in section 4.2.3, we shall briefly review the relevant findings on other systems that are related to our novel nonequilibrium interfacial one. At first, equilibrium interfacial systems will be addressed in section 4.2.1. Afterwards, we shall go into nonequilibrium one-phase systems in section 4.2.2. Both represent cut-outs of our nonequilibrium interfacial system. We can thus estimate its behaviour by "gluing together" and superimposing the results obtained for these two systems (see section 4.2.3). The so-obtained distributions of the local observables do, however, not account for the influence of the thermocapillary convection, yet. It additionally warps, so to speak, the estimated distributions. Experimental studies of thermocapillary convection (see section 2.1.1) and of similar Couette and Poiseuille flows will provide further clues.

The thermodynamic laws allow for conclusions on the global behaviour of our nonequilibrium interfacial system and its related systems even before looking at their behaviour. Thus, each of our nonequilibrium systems can be considered as an idling heat engine connected to two heat baths of different temperatures, $T_{C}$ and $T_{H}$. If they differ only moderately, it eventually assumes a stationary state featuring, among other things, a time-independent system energy, $E$, and entropy, $S, \dot{E}=0$ and $\dot{S}=0$. Then, the two absolute heat fluxes across the diathermic boundaries of the engine must be identical 
$\left|\dot{Q}_{C}\right|=\left|\dot{Q}_{H}\right|=\dot{Q}$

while its positive entropy production rate results from the difference between the entropy fluxes across them

$\dot{S}_{g e n}=\left|\dot{S}_{C}\right|-\left|\dot{S}_{H}\right|=\frac{\dot{Q}}{T_{C}}-\frac{\dot{Q}}{T_{H}}=\frac{\dot{Q}\left(T_{H}-T_{C}\right)}{T_{C} T_{H}}>0$.

Our equilibrium systems have as well closed diathermic boundaries, but they are always in contact with a single heat bath only. Hence, it exchanges no heat with the system in thermodynamic equilibrium, making also the entropy production zero,

$\dot{Q}=0$ and $\dot{S}_{g e n}=0$. 


\subsubsection{Equilibrium Interfacial Systems}

According to Gibbs's phase rule [gibbs1875], [findlay1951], two intensive thermodynamic properties, e.g. the pressure, $P$, and the temperature, $T$, of a binary two-phase equilibrium system, determine its global state, i.e. the state of the system as a whole, unambiguously. However, a local description is often more desirable, so also for this work. In order to achieve such a description, the system is subdivided into domains whose states are to be characterised. At sufficient distances from the interface such a subdomain can be described as if it were a homogeneous binary phase. When taken separately, it is characterised according to Gibbs phase rule by three intensive properties, e.g. the pressure, $P$, the temperature, $T$, and the mole-fraction, $x_{i}$, of one of the substances, $\check{l}$. A satisfactory description of the subdomains in the interfacial region, i.e. between the phases, is still subject to research. Go back to the diffuse interface models in section 2.2.2 for further details. Due to the lack of such a characterisation, the research concentrates on describing how different observables of interest vary in going from one phase to the other one. Using mainly such variations, we shall demonstrate in section 5.1.1 that our simulation systems approximate the reality. Section 4.3 will explain how we determined these observables in our simulations.

\section{Thermodynamic Observables}

In section 2.2.2 we made general remarks concerning the variation of the thermodynamic observables: Intensive ones by nature, so-called "field properties", remain constant across the interface, while "densities", i.e. quasi-intensive properties that are merely ratios of extensive observables, vary. The temperature, for instance, is a field property and must thus be constantly distributed throughout our equilibrium interfacial systems. According to van der Waals and CahnHilliard theory [cahn1958], the variation of the partial density across a liquid-fluid interface is approximated by a hyperbolic tangent function. Figure 16 shows qualitatively the hyperbolic tangent profiles to expect for the transition from an ArA phase to an ArB phase in positive $z$ direction in our equilibrium interfacial systems. The governing equations read

$$
\rho_{A r A}(z)=\frac{\rho_{A r A}(A r A)+\rho_{A r A}(A r B)}{2}-\frac{\rho_{A r A}(A r A)-\rho_{A r A}(A r B)}{2} \tanh \left(\frac{2\left(z-\zeta_{A r A}\right)}{\omega_{A r A}}\right)
$$


$\rho_{A r B}(z)=\frac{\rho_{A r B}(A r A)+\rho_{A r B}(A r B)}{2}-\frac{\rho_{A r B}(A r A)-\rho_{A r B}(A r B)}{2} \tanh \left(\frac{2\left(z-\zeta_{A r B}\right)}{\omega_{A r B}}\right)$,

where the $z$-coordinates of the Gibbs dividing surfaces are denoted as $\zeta_{A r A}$ and $\zeta_{A r B}$ while the expressions, $\rho_{A r A}(A r A), \rho_{A r A}(A r B), \rho_{A r B}(A r A)$, and $\rho_{A r B}(A r B)$, represent the partial densities in the phase rich of the substance given in brackets, e.g. $\rho_{A r B}(A r A)$ is the ArB partial density in the ArA rich phase. The interfacial widths, $\omega_{A r A}$ and $\omega_{A r B}$, are characterised by the fractions

$\omega_{A r A}=\left|\frac{\rho_{A r A}(A r A)-\rho_{A r A}(A r B)}{\frac{\partial \rho\left(\zeta_{A r A}\right)}{\partial z}}\right|$ and

$\omega_{A r B}=\left|\frac{\rho_{A r B}(A r A)-\rho_{A r B}(A r B)}{\frac{\partial \rho\left(\zeta_{A r B}\right)}{\partial z}}\right|$,

as indicated exemplarily in Figure 16. The numerators in these fractions, $\rho_{A r A}(A r A)-\rho_{A r A}(A r B)$ and $\rho_{A r B}(A r A)-\rho_{A r B}(A r B)$, also express the mutual solution of both species. The smaller the differences between both phases are, the more they are generally also alike in terms of their other observables. Depending on the variation of the partial densities in the interfacial region, the overall density, which is simply their sum, can have an extremum. In binary systems of almost immiscible substances, similar to ours, minima occur [meyer1988], [toxvaerd1995], [hafskjold1995], [geysermans2005]. They were termed "vacuous gaps" [stecki1995] and occur for formal reasons only if the Gibbs dividing surface of each substance lies closer to the phase where this substance constitutes the majority component. Thus we chose in Figure $16 z$-locations of $\zeta_{A r A}=-0.1 \mathrm{~nm}$ and $\zeta_{A r B}=0.1 \mathrm{~nm}$ for the partial density profiles with identical widths of $\omega_{A r A}=\omega_{A r A}=1.0 \mathrm{~nm}$.

The effects of various system parameters on the variations of the thermodynamic observables across the interface have already been reported in the literature [meyer1988], [toxvaerd1995], [geysermans2005]. At this point, we shall briefly dwell on the temperature effects since they are relevant for our nonequilibrium interfacial systems. Buhn et al. [buhn2004a], [buhn2004b] [buhn2006] conducted separate NPT simulations of an equilibrium interfacial system, each at a different temperature but with the pressure held constant. In these simulations, the binary mixtures, which are comparable to ours, essentially exhibit a type II behaviour in the classification by Scott et al. [scott1970] and van Konynburg et al. [vankonynburg1980] (see also part 11). In the liquid-liquid region, the interfacial width and the mutual solubilities of both substances increase at higher 
temperatures until the phase separation disappears at the upper critical solution temperature (UCST) and both phases turn into a single one. Concurrently, the numerical values of any other quasiintensive property increasingly resemble each other in both phases, up to the UCST where the values coincide. Contrariwise, they approach the values of the pure substances at lower temperatures.

\section{Hydrodynamic Observables}

According to the second law of thermodynamics (see e.g. [fermi1937]), the entropy of an equilibrium system is constant so that all local com velocities must equal the system com velocity, initially set to zero as stated in section 4.4. Since the shear stresses must thus be zero, i.e. the tensor is diagonal, only the variations of the normal stresses across an interface have been thoroughly studied in the literature [rao1979], [walton1983], [lee1984], [nijmeijer1988], [haye1994], [todd1995], [varnik2000], [hafskjold2002b]. While the normal stresses perpendicular to the interface, $S_{z z}$ in our systems, remain constant, the normal stresses lateral to the interface, $S_{x x}$ and $S_{y y}$ in our systems, both go through a minimum. Their variations must be identical due to the lateral homogeneity of the system. Hence, we shall only give their arithmetic averages, $S_{x x y y}$, if sufficient to make a point:

$S_{x x y y}=\frac{S_{x x}(z)+S_{y y}(z)}{2}$

Away from the interfaces, where the system tends to become homogeneous in all three dimensions, all normal stresses, i.e. perpendicular and lateral to the interface, have identical values close to the system pressure. The path integral of the difference between the normal stresses perpendicular and lateral to the interface produces the surface tension, $\gamma$,

$\gamma=\int_{-\infty}^{\infty} S_{z z}(z)-S_{x x y y}(z) \mathrm{d} z$

We stated already in section $\mathbf{2 . 2}$ that the local volume force, $\mathbf{f}$, is the divergence of the stress tensor, S. When applied to the microscopic definition of the stress tensor from section 4.3.2, this gradient can be rewritten as the sum of two contributions:

$\nabla \cdot \mathbf{S}=\nabla \cdot \mathbf{I}+\nabla \cdot \mathbf{K}=\mathbf{i}+\mathbf{k}=\mathbf{f}$.

We shall denote them simply as the kinetic, $\mathbf{k}$, and the interatomic, $\mathbf{i}$, force. Both must always add to zero in an equilibrium system since its stress tensor is diagonal with zero divergence:

$\mathbf{f}=\mathbf{i}+\mathbf{k}=0$. 
Thus, it holds specifically in $z$-direction of our equilibrium interfacial systems:

$k_{z}=\frac{\partial K_{z z}}{\partial z}=-\frac{\partial\left(\rho k_{B} T\right)}{\partial z}=-k_{B} T \frac{\partial \rho}{\partial z}-k_{B} \rho \frac{\partial T}{\partial z}=-i_{z}=-\frac{\partial I_{z z}}{\partial z}$.

While the second summand is zero due to the homogeneous temperature distribution, the first summand must be the negative interatomic volume force, $i_{z}$, in the equation above. Its validity is confirmed by the BGY equation (4-4), which backs the conception of the internal forces as surface forces in the interfacial region (see 2.2). We can therefore use equation (4-3) to check the consistency of our results in section $\mathbf{5 . 1}$.

Figure 16 gives an impression of how the interatomic volume $z$-forces can be expected to vary based on the hyperbolic tangent density z-profiles from the previous subsection:

$$
\begin{aligned}
& i_{z}=k_{B} T \frac{\partial \rho}{\partial z}= \\
& =k_{B} T \frac{\partial \rho_{A r A}}{\partial z}+k_{B} T \frac{\partial \rho_{A r B}}{\partial z}= \\
& = \pm k_{B} T \frac{\rho_{A r A}(A r A)-\rho_{A r A}(A r B)}{w_{A r A} \cosh ^{2}\left(\frac{2\left(z-\zeta_{A r A}\right)}{\omega_{A r A}}\right)} \pm k_{B} T \frac{\rho_{A r B}(A r A)-\rho_{A r A}(A r B)}{w_{A r B} \cosh ^{2}\left(\frac{2\left(z-\zeta_{A r B}\right)}{\omega_{A r B}}\right)} .
\end{aligned}
$$

In section 5.1.1 we shall see a similar $z$-shaped variation of the interatomic volume $z$-forces in the interfacial regions.

\section{Statistical Mechanical Observables}

The potential of mean force and its underlying mean interatomic force per particle have been studied for various equilibrium systems, see e.g. [cicotti1989], [kollman1993], [marrink1994], [pohorille1996], [billes2003]. Both observables go back to the early theories on molecular mechanics by Laplace, Young, Rayleigh, Maxwell and Young [rowlinson2002]. In particular the mean forces resemble those used to explain the cohesion of a liquid. It was later confirmed that they depend on the temperature and on the particle density as predicted by the Born-Green-Yvon (BGY) equation. It is discussed extensively in [hill1987], for instance, and reads for the interatomic force per particle, $\mathbf{t}_{\grave{l}}$, of certain substance, $\check{l}$, in a mixture [billes2003]:

$\mathbf{l}_{i}=k_{B} T \cdot \nabla \ln \rho_{i}$, 
Here, $\rho_{\check{l}}$ is the partial density of that substance. The interatomic particle force has, in analogy with the volume one above, a kinetic counterpart, $\boldsymbol{\kappa}_{i}$, in thermodynamic equilibrium:

$\boldsymbol{\varphi}_{\grave{i}}=\mathbf{l}_{i}+\boldsymbol{\kappa}_{i}=0$.

We shall compute this counterpart in our simulations by using the BGY equation (4-4) and compare the result to the interatomic particle force obtained directly in the simulation (chapter 5.1). An agreement in such comparisons will hint at a good approximation of reality.

For now, we plug the hyperbolic tangent density $z$-profiles from above into equation (4-4), to gain already an impression of how the interatomic particle $z$-forces should vary in our equilibrium interfacial systems. Thus, the $z$-component, $l_{z, A r A}$, of these forces is in the case of the first substance, call it ArA,

$$
\iota_{z, A r A}(z)=k_{B} T \frac{\partial \ln \rho_{A r A}(z)}{\partial z}=k_{B} T \frac{1}{\rho_{A r A}(z)} \frac{\rho_{A r A}(A r A)-\rho_{A r A}(A r B)}{\omega_{A r A} \cosh ^{2}\left(\frac{2 z-z_{i f}}{\omega_{A r A}}\right)} .
$$

The analogue equation for an ArB particle reads

$$
l_{z, A r B}(z)=k_{B} T \frac{\partial \ln \rho_{A r B}(z)}{\partial z}=k_{B} T \frac{1}{\rho_{A r B}(z)} \frac{\rho_{A r B}(A r A)-\rho_{A r B}(A r B)}{\omega_{A r B} \cosh ^{2}\left(\frac{2 z-z_{i f}}{\omega_{A r B}}\right)} .
$$

All other components are zero due to the homogeneity of the systems parallel to the interface:

$$
\iota_{x, A r A}=l_{y, A r A}=l_{x, A r B}=l_{y, A r B}=0 .
$$

Figure 16 shows a typical distribution of the interatomic $z$-forces to be expected. One can tell that there exists no $z$-force in the phases. At the interfaces, however, the particles are dragged back into the phase where they constitute the majority component. They feel the maximum drag off-centre on the opposite side of the interfacial region.

Note that the equation (4-3) from the previous subsection can also be derived by plugging the BGY equation into the relation between the interatomic forces per unit volume and the ones per particle from section 4.3.5:

$$
\begin{aligned}
& i_{z}=i_{z, A r A} \rho_{A r A}+i_{z, A r B} \rho_{A r B}= \\
& =k_{B} T \frac{\partial \ln \rho_{A r A}}{\partial z} \rho_{A r A}+k_{B} T \frac{\partial \ln \rho_{A r B}}{\partial z} \rho_{A r B}=
\end{aligned}
$$


$=k_{B} T \frac{\partial \rho}{\partial z}$.

Thus, the conception of the internal forces as surface forces in continuum mechanics (see section 4.3.5) remains formally correct in our equilibrium interfacial systems despite the thinness of the interfaces. Moreover, we shall generally assume that this conception holds also in our nonequilibrium systems if the processes inside proceed moderately in the sense of continuum mechanics (see section 2.2).

One obtains a free energy difference by integration of the negative interatomic force on a particle from a coordinate $z_{1}$ to the $z$-coordinate $z_{2}$ :

$-\int_{z_{1}}^{z_{2}} \iota_{z} \mathrm{~d} z=-k_{B} T[\ln \rho(z)]_{z_{1}}^{z_{2}}=-k_{B} T \ln \frac{\rho\left(z_{2}\right)}{\rho\left(z_{1}\right)}=A\left(z_{2}\right)-A\left(z_{1}\right)$.

Such differences and are misleadingly called "potentials of mean force" (PMF). Whenever we report them for a particle in this work, they shell be given with respect to that particle being located in the phase where it constitutes the majority component. Figure 16 also shows the PMF for each species:

$\Delta A_{A r A}(z)=-k_{B} T \ln \frac{\rho(z)}{\rho_{A r A}(A r A)}$

and

$$
\Delta A_{A r B}(z)=-k_{B} T \ln \frac{\rho(z)}{\rho_{A r B}(A r B)} .
$$




\subsubsection{Nonequilibrium One-Phase Systems}

Consider a binary one-phase bulk fluid under negligible gravitational influence in a closed rectangular parallelepiped configuration, two opposite boundary regions of which are maintained at different temperatures. If their difference is small, the system assumes a stationary state with approximately linear temperature and density profiles: The local temperatures decrease while the local particle overall densities increase in direction, call it $y$, of the heat flux. Concurrently, both substances slightly segregate, which involves different slopes in the partial density profiles [ludwig1856], [soret1879], [soret1881], [eilert1914]. The strength of this segregation, which is also denoted as thermal diffusion, can be characterised by the so-called Soret coefficient $S_{T}$. It is the ratio of the mole fraction gradient of one substance, call it ArA, $\partial x_{A r A} / \partial y$, and the temperature gradient $\partial T / \partial y$ divided by the equilibrium mole fractions of this substance $x_{A r A, O}$ and of the other substance, call it ArB, $x_{A r B, 0}$, [wiegand2004], [wiegand2006]:

$$
S_{T, A r A}=\frac{1}{x_{A r A, 0} x_{A r B, 0}} \frac{\partial x_{A r A} / \partial y}{\partial T / \partial y} .
$$

No convection exists and the stress tensor is diagonal with normal stresses that are homogeneously distributed on a level with the system pressure throughout the entire system.

Our nonequilibrium one-phase systems are just of the type described above when disregarding their symmetry with respect to the $x z$-plane and the unphysical behaviour near and inside the thermostated regions. Hence, we shall mainly look for the above-described temperature, density, com velocity, and normal stress distributions in the intermediate regions (between the thermostated regions) to assess to what extent their behaviour approximates reality. However, the exact characteristics of these distributions are, except for the com velocities, hard to anticipate, e.g. the slopes of the density variations. Admittedly, the linear continuum mechanics introduced in section 2.2 can describe the behaviour of such a nonequilibrium one-phase system as long as the processes inside proceed moderately. In the continuum mechanical description, three effects are said to dominate the system behaviour, namely conduction, diffusion, and the thermal diffusion [degroot1960], [haase1963], but their phenomenological coefficients are, except in mixtures of dilute gases [grew1952], hard to predict from the molecular properties of the substances involved [cohen1993]. 
Experiments and theoretical considerations show that the Soret coefficient diverges in liquids approaching the critical solution point [haase1963], [luettmer2002]. Going from liquid densities to lower ones, the Soret coefficient decreases. Little is known about the concentration dependence of the thermal diffusion, except that it must become zero in the limit of a pure substance [wittko2007]. In addition, thermal diffusion was systematically studied in NEMD simulations of characteristic equimolar binary mixtures to obtain further indications on its influences [paolini1987], [vogelsang1987], [hafskjold1995], [wold1999], [perronace2002]. Thus, the simulation results show at least qualitatively how the thermal diffusion depends on the substances, the temperature, the pressure and the equilibrium composition of the system. As a rule of thumb, the heavier substances, the "smaller" substances, and the substances with the higher interaction strengths tend to accumulate at the cold side in equimolar binary mixtures of simple liquids [hafskjold1993], [bordat2001], [reith2000]. It is, however, doubtful that all these rules apply in the same way to our nonequilibrium one-phase systems because they are far from having an equimolar overall composition. Nevertheless, the above indications on thermal diffusion are worth mentioning here because they demonstrate the applicability of our method. Thus, most NEMD simulations were boundary driven, with extremely high temperature gradients of more than $100 \mathrm{~K} / \mathrm{nm}$. Such huge magnitudes are necessary for the influence of the temperature gradients on the nonequilibrium onephase system to be detectable in the noise of a MD simulation, as stated in section 3.2.2. Still the local thermodynamic observables varied approximately linearly between the boundaries when disregarding the unphysical system behaviour due to the perturbations there.

Lacking alternatives, we estimated the characteristics of the temperature and density distributions only very crudely by analogy to the behaviour of comparable substances in similar situations. This estimation is conducted exemplarily below for the system N-Ar1Ar2-0.6-1.0-20-7091$4.74 \times 8.00 \times 9.40-100-140$, where we concentrate on the intermediate region with positive $y$ coordinates for simplicity's sake. The results apply contrariwise to negative $y$-coordinates and are of similar magnitudes in the other system. Since the distributions of the temperatures and densities are linear in $y$-direction, their values at the centre between the thermostated regions must be the arithmetic average of the temperature constraints

$$
T(2 n m)=\frac{T_{C}+T_{H}}{2}=\frac{100 K+140 K}{2}=120 K
$$

and the overall system densities

$$
\rho(2.0 \mathrm{~nm})=\frac{N_{A r A}+N_{A r B}}{L_{x} \cdot L_{y} \cdot L_{z}}=\frac{20+7091}{4.74 \mathrm{~nm} \cdot 8.00 \mathrm{~nm} \cdot 9.40 \mathrm{~nm}} \approx 19.95 \mathrm{~nm}^{-3}
$$




$$
\begin{aligned}
& \rho_{A r A}(2.0 \mathrm{~nm})=\frac{N_{A r A}}{L_{x} \cdot L_{y} \cdot L_{z}}=\frac{20}{4.74 \mathrm{~nm} \cdot 8.00 \mathrm{~nm} \cdot 9.40 \mathrm{~nm}} \approx 0.056 \mathrm{~nm}^{-3} \\
& \rho_{A r B}(2.0 \mathrm{~nm})=\frac{N_{A r B}}{L_{x} \cdot L_{y} \cdot L_{z}}=\frac{7091}{4.74 \mathrm{~nm} \cdot 8.00 \mathrm{~nm} \cdot 9.40 \mathrm{~nm}} \approx 19.89 \mathrm{~nm}^{-3} .
\end{aligned}
$$

Concurrently, the difference between the temperature constraints, divided by the distance of the thermostated regions, delivers the temperature $y$-gradient

$$
\frac{\partial T}{\partial y}=\frac{T_{H}-T_{C}}{L_{y} / 2}=\frac{140 K-100 K}{80 \mathrm{~nm} / 2}=10.00 \frac{\mathrm{K}}{\mathrm{nm}} .
$$

It is related to the overall densities via the thermal expansion coefficient of the mixture, $\hat{\alpha}$ :

$$
\frac{\partial \rho}{\partial y}=\frac{\partial \rho}{\partial T} \frac{\partial T}{\partial y}=-\hat{\alpha} \rho \frac{\partial T}{\partial y}
$$

Since our Ar-like particles are hardly soluble, we can assume that this coefficient is of the order of the experimental one of pure Argon, $\hat{\alpha}=-0.004 \mathrm{~K}^{-1}$ [stewart1989], at the conditions determined in the corresponding equilibrium interfacial system from the previous section, i.e. $T \approx 120 \mathrm{~K}$ and $P \approx 30 \mathrm{MPa}$ (see Tables 14, 24, and 33), so that

$$
\frac{\partial \rho}{\partial y}=-\hat{\alpha} \rho \frac{\partial T}{\partial y} \approx-0.004 \frac{1}{K} \cdot 19.95 \frac{1}{\mathrm{~nm}^{3}} \cdot 10.00 \frac{\mathrm{K}}{\mathrm{nm}}=-0.80 \frac{1}{\mathrm{~nm}^{4}} .
$$

Hence the governing linear equations of the temperature and density distributions should be approximately,

$$
\begin{aligned}
& T(y)=\frac{\partial T}{\partial y}(y-2 n m)+T(2.0 n m)=10 \frac{K}{n m}(y-2 n m)+120 K \\
& \rho(y)=\frac{\partial \rho}{\partial y}(y-2 n m)+\rho(2.0 n m)=-0.8 \frac{1}{n^{4}}(y-2 n m)+19.95 \frac{1}{n^{3}},
\end{aligned}
$$

if we chose the values at the centre between the thermostated regions as points of reference.

The above-mentioned low mutual solubility of our Ar-like particles allows also only for weak thermal diffusion. In addition, the positive temperature dependence of this solubility (see section 4.2.1) tells us that the majority component partial density must decrease more strongly in $y$ direction than the overall density, while the minority component partial density must increase. The maximum increase can be estimated by assuming that the partial density of the minority component rises from zero at the cold thermostated region to the overall system partial density at the centre between the thermostated regions: 


$$
\begin{aligned}
& \rho_{A r A}(y)=\frac{\rho_{A r A}(2.0 n m)}{L_{y} / 4}(y-2 n m)+\rho_{A r A}(2.0 n m)= \\
& =0.028 \frac{1}{n m^{4}}(y-2 n m)+0.056 \frac{1}{n^{3}} .
\end{aligned}
$$

The corresponding minimum decrease of the majority component is:

$$
\rho_{A r B}(y)=\rho(y)-\rho_{A r A}(y)=0.77 \frac{1}{n m^{4}}(y-2 n m)+19.89 \frac{1}{n m^{3}} .
$$

We shall primarily look for these temperature and density $y$-variations in our simulation results (see section 5.1.2). Estimates of the other observables to be determined in our simulations can be made at this point by plugging equations (4-9) and (4-10) into equation (2-7). Thus the kinetic contributions to the normal stresses, $K_{x x}, K_{y y}$, and $K_{z z}$, must decrease in going from the cold to the hot thermostated region:

$$
\begin{aligned}
& K_{x x}=K_{y y}=K_{z z}=-\rho k_{B} T= \\
& =-\left(-0.80 \frac{1}{n m^{4}}(y-2 n m)+19.95 \frac{1}{n^{3}}\right) \cdot 1.38 \cdot 10^{-23} \frac{J}{K} \cdot\left(10 \frac{K}{n m}(y-2 n m)+120 K\right)= \\
& =-0.0138 \frac{p N \cdot n m}{K}\left(-8 \frac{K}{n^{5}}(y-2 n m)^{2}+103.5 \frac{K}{n^{4}}(y-2 n m)+2394 \frac{K}{n^{3}}\right)= \\
& =O(-33 M P a) .
\end{aligned}
$$

Since the first summand in the brackets is small compared to the other ones, we can expect that the decrease is approximately linear. The interatomic contributions to the normal stresses, $I_{x x}, I_{y y}$, and $I_{z z}$, must increase with the same absolute slope so that both contributions always add up to the system pressure, $P$ (see Tables 14, 24, and 33):

$$
I_{x x}=I_{y y}=I_{z z}=-P+\rho k_{B} T=O(0 M P a) .
$$

The divergence of the kinetic and interatomic contributions to the normal stresses results in the related volume forces (see equation (4-2)), which must always add up to zero in a stationary nonequilibrium one-phase system. It holds specifically in $y$-direction:

$$
\begin{aligned}
& -i_{y}=k_{y}=\frac{\partial K_{y y}}{\partial y}=-\frac{\partial\left(\rho k_{B} T\right)}{\partial y}=-k_{B} T \frac{\partial \rho}{\partial y}-k_{B} \rho \frac{\partial T}{\partial y}= \\
& =-1.38 \cdot 10^{-23} \frac{J}{K}\left(10 \frac{K}{n m}(y-2 n m)+120 K\right) \cdot\left(-0.8 \frac{1}{n m^{4}}\right)-
\end{aligned}
$$




$$
\begin{aligned}
& -1.38 \cdot 10^{-23} \frac{\mathrm{J}}{K}\left(-0.80 \frac{1}{\mathrm{~nm}^{4}}(y-2 \mathrm{~nm})+19.95 \frac{1}{\mathrm{~nm}^{3}}\right) \cdot 10 \frac{\mathrm{K}}{\mathrm{nm}}= \\
& =-0.0138 \frac{\mathrm{pN} \cdot \mathrm{nm}}{\mathrm{K}}\left(-16 \frac{\mathrm{K}}{\mathrm{nm}^{5}}(y-2 \mathrm{~nm})+103.5 \frac{\mathrm{K}}{\mathrm{nm}^{4}}\right)=O\left(-3 \frac{\mathrm{pN}}{\mathrm{nm}^{3}}\right) .
\end{aligned}
$$

Note that the kinetic volume $y$-forces always point towards the nearest cold thermostated region while the (real) interatomic ones point, on the chronological average, towards the nearest hot thermostated region. They can be rewritten as the sum of the partial densities multiplied by the interatomic forces per particle of the same substance:

$$
i_{y}=+k_{B} T \frac{\partial \rho_{A r A}}{\partial y}+k_{B} \rho_{A r A} \frac{\partial T}{\partial y}+k_{B} T \frac{\partial \rho_{A r B}}{\partial y}+k_{B} \rho_{A r B} \frac{\partial T}{\partial y}=\rho_{A r A} \cdot l_{y, A r A}+\rho_{A r B} \cdot l_{y, A r B} \cdot(4-
$$

This leads to the numerical estimates

$$
\begin{aligned}
& l_{y, A r A}=+k_{B}\left(\frac{T}{\rho_{A r A}} \frac{\partial \rho_{A r A}}{\partial y}+\frac{\partial T}{\partial y}\right)= \\
& =1.38 \cdot 10^{-23} \frac{J}{K}\left(\frac{10 \frac{K}{n m}(y-2 n m)+120 K}{0.028 \frac{1}{n m^{4}}(y-2 n m)+0.056 \frac{1}{n^{3}}} 0.028 \frac{1}{n^{4}}+10 \frac{K}{n m}\right)=O(1 p N)(4-16)
\end{aligned}
$$

and

$$
\begin{aligned}
& l_{y, A r B}=+k_{B}\left(\frac{T}{\rho_{A r B}} \frac{\partial \rho_{A r B}}{\partial y}+\frac{\partial T}{\partial y}\right)= \\
& =1.38 \cdot 10^{-23} \frac{J}{K}\left(\frac{10 \frac{K}{n m}(y-2 n m)+120 K}{0.77 \frac{1}{n m^{4}}(y-2 n m)+19.89 \frac{1}{n^{3}}} 0.77 \frac{1}{n^{4}}+10 \frac{K}{n m}\right)=O(0.2 p N) .
\end{aligned}
$$

One can tell by plugging in the extreme variations of the partial densities (equations (4-11) and (4-12)) that both interatomic particle $y$-forces must be positive, the ones per particle of the minority component being much stronger. We shall use equations (4-12), (4-14), and (4-15) to check the consistency of our simulation results in section 5.1.2. 


\subsubsection{Nonequilibrium Interfacial Systems}

Estimating the intensity of the thermocapillary convection is difficult. After all, its underlying effect still lacks a general description, as mentioned in part 1. We can, however, safely assume laminar thermocapillary flows. Turbulent ones are, a priori, unlikely, since they come only with extreme shear rates on the small length scales typically computable in a MD simulation. The results in other systems, specifically designed to study shear flows, back this requirement. Thus, several authors implemented, for instance, Couette- or Poiseuille flows in NEMD simulations [ashurst1975], [trozzi1984], [hannon1986], [cieplak1999], [muellerplathe1999], [cieplak2000], [bordat2002]. They are mostly boundary driven, with extreme velocity gradients of up to $\partial u / \partial y=10 \cdot 10^{10} \mathrm{~m} / \mathrm{s}$. One applies them although they make an additional thermostating necessary to balance the energy dissipation. Otherwise, their influence would not become noticeable against the noise in the com velocity distribution. Nevertheless, the system behaviour approximates reality at sufficient distances from the boundaries. Several authors obtain, for instance, results on the dynamic viscosities which lie close to those determined in experiments [bordat2002], [hannon1986], [trozzi1984]. Turbulent flows, however, occur in none of the abovementioned simulations.

Although we already expected laminar flows, we estimated, for later comparison to our results, a conservative maximum shear rate up to which the flows remain definitely laminar. Consider, for instance, a fully developed turbulence. It consists, in the sense of Richardson [richardson1922], Kolmogorov and Obukhov [kolmogorov1941a], [obukhov1941b] [kolmogorov1991], [obukhov1941], of a cascade of superposed eddies with different scales of motion. Larger eddies pass their kinetic energy to smaller and smaller ones until viscous dissipation becomes important in them. Such dissipative eddies have a characteristic dimension of $\lambda_{0}$. It is the one, $l$, in the largest eddies divided by their Reynolds number, $R e$, to the power of 0.75 [landau2003] [pope2003]:

$\lambda_{0}=l / \operatorname{Re}^{3 / 4}$

This Reynolds number involves their aforementioned characteristic length, their velocity variation, $\Delta v$, as well as the fluid density, $\rho$, and dynamic viscosity, $\tilde{\eta}$ :

$\operatorname{Re}=\frac{\bar{\rho} l \Delta v}{\tilde{\eta}}$ 
We naively set $\lambda_{0}$ to $l$ in equation (4-18), i.e. $R e=1$, to be on the safe side. Then, $\Delta v$ must be larger than

$$
\Delta v=\frac{\operatorname{Re} \eta}{l \bar{\rho}}=\frac{1 \cdot 100 \cdot 10^{-6} \frac{\mathrm{kg}}{\mathrm{sm}}}{100 \cdot 10^{-10} \mathrm{~m} \cdot 1000 \frac{\mathrm{kg}}{\mathrm{m}^{3}}} \approx 10 \frac{\mathrm{m}}{\mathrm{s}}
$$

for the smallest eddies to fit into our systems. Equation (4-19) uses their typical properties for the sake of simplicity. The dimension of the smallest eddies is typically a fraction of that of the largest eddies. Hence, $\Delta v$ must in fact be much larger.

Having established the conditions for the occurrence of the laminar thermocapillary flows, we shall now consider their appearance. They should run from hot to cold along the interfaces, as in the vast majority of experimental studies (see section 2.1.1). Since the different flows cannot penetrate each other, eight roll cells must occur, compatible with the periodic boundary conditions. In the below discussions of the simulation results on the nonequilibrium interfacial systems (see section 5.1), we shall look for such roll cells as a confirmation for the occurrence of any "thermocapillary convection".

Note, how the distribution of the velocity components parallel to the interfaces resembles in each phase a Couette-Poiseuille flow [spurk1996]. This resemblance increases the larger the distance between the thermostated regions may be: The interfaces represent two confining walls that move in direction of the temperature gradient. As a result, a pressure gradient in opposite direction builds up. It drives the counter flows at the centres between the interfaces. The Knudsen effect in dense gases has the same underlying mechanism when disregarding the different directions of the flows (see section 2.1.2). We shall also look in the discussions of our systems for such pressure differences in connection with the thermocapillary convection.

Later, also the intensity of the convection will be discussed as function of different system parameters (see e.g. chapter 5.4). In practice, measuring such an intensity comes with several difficulties, however. We shall simply assess it by using the maximum absolute com velocity components in the system. Moreover, we shall compute also the spatial correlation of the velocities, $\mathbf{v}$, in the style of Ramirez et al [ramirez2000]:

$$
C=\sqrt{\sum_{\forall i, j}\left[\sum_{i^{\prime}, j^{\prime}} \mathbf{v}\left(\alpha^{\prime}, \beta^{\prime}\right) \cdot \mathbf{v}\left(\alpha^{\prime \prime}, \beta^{\prime \prime}\right)\right]} .
$$


Here, the first sum runs over all subvolumes, $\left(\alpha^{\prime}, \beta^{\prime}\right)$, in a system while the second one includes their applicable eight first neighbours, $\left(\alpha^{\prime \prime}, \beta^{\prime \prime}\right)$.

In addition to the convection and its dependencies, we shall check in the discussion of the results whether the distributions of the other local observables are plausible. Remember from section $\mathbf{2 . 2}$ that continuum mechanics can describe a system if the processes inside proceed moderately. Since this condition will turn out to be fulfilled for the equilibrium interfacial system and the nonequilibrium one-phase systems in chapter 5.1, we can expect that the same holds also for the nonequilibrium interfacial system. In the continuum mechanical description the above convection constitutes another transport mechanism besides the molecular ones, namely conduction, diffusion, and thermal diffusion, introduced previously for the nonequilibrium one-phase system.

Disregard the thermocapillary convection for the moment and think only about the molecular transport mechanisms. Then, the distribution of the local observables should appear approximately as a combination of the distributions in two nonequilibrium one-phase systems (see section 4.2.2), one for each phase, joined by various distributions of equilibrium interfacial systems (see section 4.2.1) in slabs perpendicular to the temperature gradient. That is, while each phase separately strives to mimic a stationary nonequilibrium one-phase system, both phases together strive to establish local phase equilibria in slabs parallel to the $x z$-plane as function of the local temperature there. Based on these two competing tendencies, an intermediate distribution of the local thermodynamic observables should appear that is mainly a function of $y$ (direction of the temperature gradient) and only at the interface a function of $z$ (perpendicular to the interface).

If we now also take the thermocapillary convection into consideration, the convective transport "warps" the distribution of the local thermodynamic observables described before. Having only a crude idea on the intensity of the thermocapillary convection, it is difficult to anticipate the magnitude of this perturbation. After all, convective transport rates are generally substantial in comparison to molecular ones. However, we must also take the short length scales of the MD simulation into consideration. The empirical dimensionless Péclet number, $P e$, for instance, describes the relative influence of molecular and convective heat transport on the behaviour of a given system [bird2002]:

$$
P e=\frac{l \cdot v \cdot \bar{\rho} \cdot c_{P}}{\lambda_{t h}}
$$


Here, $l$ and $v$ are the characteristic length and velocities of the system, while $\rho, c_{P}$, and $\lambda_{\text {th }}$ represent the density, heat capacity at constant pressure and thermal conductivity of the fluid. In the nonequilibrium interfacial system, all these variables are either material properties or externally constrained, except for $v$. We shall assume that the roll cells are approximately an eighth of the system $z$-dimension wide, $l=1 \mathrm{~nm}$. Then the order of the Péclet number can be roughly estimated as function of $v$ with the help of the experimental material properties of Argon [vasserman1967], [younglove1986], [richard1989], [gilgen1994], [tegeler1999]:

$$
P e=\frac{1 \cdot 10^{-9} \mathrm{~m} \cdot \mathrm{v} \cdot 1000 \frac{\mathrm{kg}}{\mathrm{m}^{3}} \cdot 1000 \frac{\mathrm{J}}{\mathrm{kgK}}}{100 \cdot 10^{-3} \frac{\mathrm{W}}{\mathrm{mK}}}=0,01 \frac{\mathrm{s}}{\mathrm{m}} \cdot \mathrm{v} .
$$

Accordingly, for convective transport to be substantial in the thermocapillary system, the characteristic velocity of the roll cells must be on the order of $100 \mathrm{~m} / \mathrm{s}$ or higher. Since the roll cells cover the entire system, the local thermodynamic observables in the phases should become $z$ dependent in this case. Provided the expected thermocapillary convection does occur, our second indication for the soundness of the MD simulation will therefore be to see if the characteristic velocity of the roll cells agrees with the distributions of the local observables in the phases. 


\subsection{Analysis of our "Simulation Systems"}

As explained in part 3, observables (in equilibrium: thermodynamic averages) are typically computed from the simulated sample of the phase space trajectory by suitable averaging procedures. Their convergence strongly differs for each observable; single particle properties being usually much easier to obtain than collective ones. Only few observables converge sufficiently well in the small time-space window necessary to describe the macroscopic behaviour in a system. In this work, we determined, for simplicity's sake, solely "static" (i.e. time independent) observables. Their numerical values become more accurate the more particles are taken into consideration and the longer they are monitored. Most of them are determined locally in our simulations. By "local observables" we understand here averages that are computed only over the particles in small spatially fixed subvolumes of a simulation system to catch its local character. In contrast, the properties of all particles enter into the "global observables" of a system. Although they fail to indicate the occurrence of the thermocapillary convection, we monitored them nevertheless to ensure the comparability of the simulations.

Besides the computing power, the memory capacity is another important limiting factor in MD simulations. If we stored, according to common practice, the computed phase points during the simulation for subsequent analyses, the amount of data would quickly skyrocket. A single phase point, i.e. particle positions, momenta, (and possibly forces), requires approximately 250 kilobytes in a system of 7000 particles. Here, it proves advantageous that we confined ourselves to determine only static observables. The peculiarity of their definitions (see part 3) makes it often practical to determine them "on the fly" during the simulations. To do so, the instantaneous function values $o(\mathbf{q}(t), \mathbf{p}(t))$ underlying the observable, $o$, must be determined right after the computation of each phase point. The numerical values $\mu(o)$ can then be computed by suitable implementation of the following formula

$\mu(o)=\frac{1}{n} \sum_{m=1}^{n} o\left(\mathbf{q}\left(t_{m}\right), \mathbf{p}\left(t_{m}\right)\right)$

where $\left(\mathbf{q}\left(t_{m}\right), \mathbf{p}\left(t_{m}\right)\right)$ represents the phase points at the considered times, $t_{m}$, while $n$ is the running number of phase points considered so far. Hence, the individual phase points themselves need not be stored, which is, of course, at the expense of slightly longer run-times. 
In order to keep these run-times manageable, only those phase points were furthermore taken into account considered that are chronologically separated by roughly the relaxation time of the observables under consideration. We can accept the thus resulting loss of information due to the strong correlation of the function values within this time. The relaxation times of the most observables in this work lie typically on the order of a few hundred to thousand time steps, i.e. in our case with Argon masses, of the order of picoseconds [haile1997]. We considered only every thousandth time step in the case of the global observables and every hundredth time step in the case of the local observables. Our production runs include typically $75 \mathrm{~ns}$ long ( $15 \cdot 10^{6}$ time steps). Those of the simulation system under the default constraints took, for instance, up to $300 \mathrm{CPU}$ hours on our computers.

The obtained observables have little significance for the system behaviour unless the noise in them is known. It is generally assessed through studies of the correlations in the instantaneous function values that underly the observables [straatsma1986]. Since we decided to determine them on-thefly, however, such studies involve substantial effort. Instead, we preferred to estimate the noise in two comparatively coarse ways described in the literature [haile1997], [rapaport2004]. On the one hand, we computed along with the observables $o$, i.e. the means, $\mu(o)$, also the standard deviations, $\sigma(o)$, of the instantaneous function values, $o(\mathbf{q}(t), \mathbf{p}(t))$, considered (see above):

$\sigma(o)=\sqrt{\frac{\sum_{m=1}^{n}\left[o\left(\mathbf{q}\left(t_{m}\right), \mathbf{p}\left(t_{m}\right)\right)\right]^{2}-\left[\sum_{m=1}^{n} o\left(\mathbf{q}\left(t_{m}\right), \mathbf{p}\left(t_{m}\right)\right)\right]^{2}}{n-1}}$.

These standard deviations provide only crude estimates of the fluctuations in the related means, since we lack the correlation coefficients. Nevertheless, wet manage to catch in this way the qualitative differences between similar systems or, in the case of the local observables, even variations within the same system quite well (see section 5.1). On the other hand, we determined several values, $\mu_{s}(o)$, for the observables over consecutive segments of the whole phase space trajectory computed in a simulation. If these segments are long compared to the relaxation times of the observables, their values are statistically independent. The standard deviations of the several values $\sigma_{s}(o)$ then allow to estimate the noise in the observable, $o$, determined from the whole computed phase space trajectory: 
$\sigma\left(\mu_{s}(o)\right)=\sqrt{\frac{\sum_{s=1}^{n}\left[\mu_{s}(o)\right]^{2}-[\mu(o)]^{2}}{n-1}}$.

We typically divided the trajectory into $n=3$ segments. Such a division comes without extra costs because we had our simulation code export the (intermediate) results anyhow on a regular basis to reduce the severity of a potential computer failure. Concurrently, we make sure in this way that the length of each segment, typically $25 \mathrm{~ns}\left(5 \cdot 10^{6}\right.$ time steps), clearly exceeds the above relaxation times of our observables. Some scattering in the thus obtained standard deviations is acceptable. In the case of the local observables, these segmental standard deviations were additionally spatially averaged to obtain single characteristic values for the entire system.

Several static observables were determined together with their standard deviations in the course of this work. For the sake of simplicity, we shall divide them up into three groups according to their historical origins:

- thermodynamic observables: Particle overall and partial densities, temperatures, system pressures, internal system energies (see section 4.3.1);

- hydrodynamic observables: Centre of mass (or in brief "com-") velocities, linear system momenta, selected stress tensor elements, surface tensions (see section 4.3.2);

- statistical mechanical observables: Forces (see section 4.3.3).

Each section contains a brief discussion of the instantaneous function values $o(\mathbf{q}(t), \mathbf{p}(t))$ underlying the observables. Although their statistical mechanical definitions hold, strictly speaking, only in thermodynamic equilibrium, we applied them likewise to our nonequilibrium systems, assuming that the processes proceed moderately (see section 3.1). In doing so, we also neglected the bias of the particle velocity distribution in consequence of the thermocapillary convection [evans1990]. In addition to the observables from the three aforementioned groups, the overall heat in- $\left(\dot{Q}_{H}\right)$ and outfluxes $\left(\dot{Q}_{C}\right)$ of the nonequilibrium systems per unit time were determined together with the related standard deviations. The instantaneous function values, $o(\mathbf{q}(t), \mathbf{p}(t))$, underlying these fluxes equal the instantaneous rates of kinetic energy coupled into or out of each "thermostated region",

$$
\begin{aligned}
& \dot{Q}_{C}=\Delta K_{C}=\Delta K_{x, C}+\Delta K_{y, C}+\Delta K_{z, C} \text { and } \\
& \dot{Q}_{H}=\Delta K_{H}=\Delta K_{x, H}+\Delta K_{y, H}+\Delta K_{z, H} .
\end{aligned}
$$

See section 4.1.4 for more details on how these rates are calculated. 
Before the local observables could be determined their locations and spatial resolution had to be chosen so as to catch the spatial variation of the system in consideration. Both the locations and the resolution are specified through the way of dividing the system into the subvolumes or of the positioning of the cut-surfaces. They represent the references for which the local observables are computed. We divided each of our systems evenly into adjoining subvolumes that do not spread across its boundaries. Depending on the type of a system, the division was conducted so as to obtain slabs or cuboids. In either case, we explored suitable subvolume dimensions in a series of production simulations of the first Ar1Ar2 interfacial systems under the default constraints. Both the division and the exploration will be explained more in detail in see section 4.3.4. When interpreting the results later in this work, we must keep in mind that the system behaviour is, depending on the distance from the thermostated regions, more or less unphysically perturbed by the manipulation of the particle velocities there (see section 3.2.2). This behaviour can be seen particularly well in our nonequilibrium one-phase systems which, due to their homogeneities, allow using slabs perpendicular to the y-direction. We can thus achieve in it a high spatial resolution. Our assumption is, based on the results for such systems in section 4.3.4, that the unphysical behaviour fades to insignificant levels at a distance of about $1 \mathrm{~nm}$ from the centre of the thermostated regions. We will also establish in the same section, however, that the error in the data at the thermostated regions can be expected to be small if the local observables are determined using the relatively coarse cuboids. In order to determine the spatial variation of selected stress tensor elements, we shall position cut-surfaces at equal distances into the equilibrium systems. Suitable distances were obtained from another series of production simulations of the Ar1Ar2 equilibrium interfacial system (see also 4.3.4). In this series, we also determined a suitable value for the normal distance, $\delta \alpha$, from the cut surfaces within which the particles are considered in the calculation of the kinetic contribution. A drift of our systems, that would interfere with the determination of the local observables, could not be detected.

The results as obtained directly from our simulations are still partly unsuitable for our studies in part 5. Hence, we summarised particularly the local data by a series of measures first (see section 4.3.5). Thus, the volume forces related to the particles of a certain substance were converted into more meaningful forces per particle. In the interfacial systems, the distributions of the local observables were additionally relocated to ease their comparison. Finally, we averaged redundant local data and derived, whenever possible, additional properties from them to obtain a better understanding. Such "derivational properties" are, for instance, locations and widths of the interfaces, Soret coefficients, 
the surface tensions, or gradients. They were computed from the averaged data according to the applicable definitions in chapter 4.2. Moreover, we shall give explicitly the maximum absolute local $y$ - and z-com velocity components as a measure for the intensity of the convection. Section $\mathbf{5 . 1}$ will discuss exemplarily also all the non-averaged local data so that the reader may assure himself of their quality. Subsequently, we shall report the results right away in the summarised form and discuss, for brevity's sake, only those necessary to make a point.

In addition to the abovementioned local data, we averaged also the global heat fluxes. Their both absolute values must be a priori identical in each of our stationary nonequilibrium systems. Hence, we shall give in Tables 14, 24, and 33 once again the averaged absolute heat fluxes,

$Q=\frac{\left|Q_{C}\right|+\left|Q_{H}\right|}{2}$

They should apply as well within the related systems across any plane perpendicular to the $y$-axis. Moreover, Tables 11, 21, and 30 give the thermal pseudo-conductivities, $\lambda_{t h}$. We shall call them pseudo because they are computed by disregarding a possible convection in the systems. Accordingly, these conductivities result simply from the heat fluxes, the thermostated temperatures, and the system dimensions according to the relation

$\lambda_{t h}=\frac{Q}{\left(T_{H}-T_{C}\right) L_{z} \cdot L_{x} \cdot L_{y}}$. 


\subsubsection{Thermodynamic Observables}

The instantaneous particle densities are defined as the number of particles inside a region $\delta V$, divided by its volume [allen2003]. While the particle overall densities comprise the total number, $N$, of all particles in that region

$$
\rho=\frac{N}{\delta V}
$$

the particle partial densities always refer to a single "substance", $\check{\imath}$, i.e. only the total number of particles of that substance, $N_{\check{i}}$, is counted

$\rho_{\check{l}}=\frac{N_{\check{l}}}{\delta V}$

Instantaneous temperatures were determined both, locally and globally. We define them at each time step through the relation [mcquarrie2000]

$T=\frac{2}{3} \frac{K}{(N-\hat{c}) k_{B}}=\frac{2}{3 k_{B}} \frac{\sum_{j \in \delta V} \frac{1}{2} m_{j}\left|\mathbf{u}_{j}\right|^{2}}{N-\hat{c}}$.

It bases on the assumption that the particle velocity distribution is sufficiently close to a MaxwellBoltzmann one. In the definition, $k_{B}$ is Boltzmann's constant, while $m_{j}$ and $\mathbf{u}_{j}$ are the mass and the velocity of the individual particles, $j$, inside the considered region, $\delta V$. The sum of their kinetic energies over all particles, $N$, has the symbol $K$. When determining the temperatures globally, we reduced $N$ by one, i.e. $\hat{c}=1$, to account for the constraints on the particle degrees of freedom through the periodic boundaries of our systems. Otherwise, we simply neglected these constraints and set $\hat{c}=0$. The error resulting from the neglect is less than $1 \%$.

The instantaneous pressure and the internal energy of our systems were computed according to their common definitions in MD simulations, see e.g. [allen2003]. For the pressures, the virial route was used. Corrections for the long range interactions in the configurational energy or in the pressure were neglected because they are difficult to consider in heterogeneous systems. 


\subsubsection{Hydrodynamic Observables}

The instantaneous centre of mass (com-) velocity was determined both, locally and globally. It is the sum of the linear momenta, $\mathbf{p}_{j}$, of all particles $j$ inside a region, $\delta V$, divided by the total number of particles, $N$, inside that region [irving1950]

$\mathbf{v}=\frac{\sum_{j \in \delta V} \mathbf{p}_{j}}{N}$.

In addition to this general com velocity, we also determined ones specifically for each substance, $\check{l}$ :

$\mathbf{V}_{\grave{\imath}}=\frac{\sum_{i=\delta V, i} \mathbf{p}_{j}}{N_{\check{l}}}$.

Here, $N_{i}$ represents its total particle number. Such specific velocities describe the relative motion of the different particles. Molecular diffusion involves, for instance, such motions.

As stated previously, the stress tensor, $S_{i^{\prime} j^{\prime}}(\mathbf{r})$, refers to three mutually perpendicular cut-surfaces, $\delta S_{j^{\prime}}$. They lie perpendicular to the coordinate axes, $j^{\prime}$, and intersect at the location, $\mathbf{r}$. The value of the stress tensor consists of two additive contributions. We defined the kinetic one already in equation (2-5). One could call the other contribution, $I_{\mathrm{i}^{\mathrm{j} j}}(\mathbf{r})$, interatomic. It accounts for the interand intramolecular forces that act across these cut surfaces in dense fluids. Its precise definition remains, however, subject to research since an unambiguous way of assigning such forces to the different cut-surfaces has not been found yet [kirkwood1949b], [harasima1958], [schofield1982], [rowlinson2002]. For the sake of simplicity, we confined ourselves here to the presumably most widely used expression, suggested by Irving and Kirkwood [irving1950]:

$I_{i^{\prime} j^{\prime}}(\mathbf{r})=-\frac{1}{2} \sum_{j=1}^{N} \mathbf{l}_{j} \cdot \mathbf{e}_{i^{\prime}} \operatorname{sgn}\left(\left(\mathbf{r}_{j}-\mathbf{r}\right) \cdot \mathbf{e}_{j^{\prime}}\right)$.

Equation (4-20) has already been rewritten so as to apply for our molecular model and forces field [todd1995]. Similar to the kinetic contribution above, the multiplication with -1 makes the definition compatible with the hydrodynamic conception of the stresses tensor (see section 2.2). We sum over all particles, $N$, inside the system. A considered particle, $j$, has the location, $\mathbf{r}_{j}$, and feels the net force, $\mathbf{l}_{j}$, from the interactions with all other particles. The expression above can be rewritten into an easier to implement sum 


$$
I_{i^{\prime} j^{\prime}}(\mathbf{r})=-\frac{1}{\delta S_{j^{\prime}}} \sum_{n} \operatorname{sgn}\left(\mathbf{r}_{i j} \cdot \mathbf{e}_{j^{\prime}}\right) \frac{-\partial u\left(r_{i j}\right)}{\partial\left(r_{i j}\right)} \frac{\mathbf{r}_{i j}}{r_{i j}} \cdot \mathbf{e}_{i^{\prime}}
$$

over all pair interactions $n \in\left\{r_{i j} \cap \delta S_{j^{\prime}}\right\}$ across the cut surface $\delta S_{j^{\prime}}$ [heinz2005]. The particles involved in each pair interaction are generally denoted by $i$ and $j$ here. Their connecting vector carries the symbol, $\mathbf{r}_{i j}=\mathbf{r}_{j}-\mathbf{r}_{i}$, and has the length, $r_{i j}$.

The cut surface areas required to catch the local character in a strongly heterogeneous system are so small that it becomes almost impossible to obtain reliable values for the related stress tensor elements. Since our equilibrium systems are homogeneous parallel to the interfaces, however, at least some of the stress tensor elements can still be determined [todd1995], [ikeshoji2004]. Thus, we computed following previous works [rao1979], [lee1984], [walton1983], [nijmeijer1988], [varnik2000] the stresses at different cut surfaces parallel to the interfaces. The normal stresses, $S_{z z}$, are:

$$
S_{z z}(\mathbf{r})=-\frac{1}{\delta V} \sum_{n \in \delta V} m_{n}\left(\mathbf{u}_{n} \cdot \mathbf{e}_{z}\right)^{2}-\frac{1}{\delta S_{z}} \sum_{n} \operatorname{sgn}\left(z_{j}-z_{i}\right) \frac{-\partial u\left(r_{i j}\right)}{\partial\left(r_{i j}\right)} \frac{z_{i j}}{r_{i j}} .
$$

Moreover, based on a mathematical rearrangement in the definition of the stress tensor [ono1960], the normal stresses lateral to the interface $\left(S_{x x}\right.$ and $\left.S_{y y}\right)$ were also computed using these cut-surfaces

$$
\begin{aligned}
& S_{x x}(\mathbf{r})=-\frac{1}{\delta V} \sum_{n \in \delta V} m_{n}\left(\mathbf{u}_{n} \cdot \mathbf{e}_{x}\right)^{2}-\frac{1}{\delta S_{z}} \sum_{n} \operatorname{sgn}\left(z_{j}-z_{i}\right) \frac{-\partial u\left(r_{i j}\right)}{\partial\left(r_{i j}\right)} \frac{z_{i j}}{r_{i j}} \frac{x_{i j}^{2}}{z_{i j}^{2}} \\
& S_{y y}(\mathbf{r})=-\frac{1}{\delta V} \sum_{n \in \delta V} m_{n}\left(\mathbf{u}_{n} \cdot \mathbf{e}_{y}\right)^{2}-\frac{1}{\delta S_{z}} \sum_{n} \operatorname{sgn}\left(z_{j}-z_{i}\right) \frac{-\partial u\left(r_{i j}\right)}{\partial\left(r_{i j}\right)} \frac{z_{i j}}{r_{i j}} \frac{y_{i j}^{2}}{z_{i j}^{2}} .
\end{aligned}
$$

Here, $x_{i j}, y_{i j}$, and $z_{i j}$ are the components of the vector, $\mathbf{r}_{i j}$, connecting the particles $i$ and $j$ through the cut surface $\delta S_{z}$. We can already tell from the denominators that the mathematical rearrangement promotes numerical noise. They contain the $z$-distance between both interacting particles. It can quite often become very small during a simulation, when their connecting vector lies almost parallel to the cut surface. We then obtain exceptionally large results. They manifest themselves particularly in the insular peaks that show up in some distributions of the lateral stresses. See e.g. Figure $34 \mathrm{~m}$. 


\subsubsection{Statistical Mechanical Observables}

Besides the density and the com velocity of a region $\delta V$, we also computed the instantaneous interatomic forces, $\mathbf{i}$, per unit volume that act on the particles inside that region. In doing so, we distinguished the particles by their types so that three volume force vectors are obtained in our binary systems of ArA and ArB:

$\mathbf{i}_{A r A}=\frac{1}{\delta V} \sum_{j \in \partial V \wedge A r A} \mathbf{l}_{j}$

$\mathbf{i}_{A r B}=\frac{1}{\delta V} \sum_{j \in \delta V \wedge A r B} \mathbf{l}_{j}$

$\mathbf{i}=\mathbf{i}_{A r A}+\mathbf{i}_{A r B}$.

Here, $\mathbf{~}_{j}$ is the net force acting on the particle, $j$, due to its interactions with all other particles.

Although it is irrelevant for the determination of these forces, we would like to note already at this point that the first two volume forces above were converted into mean forces per particle, $\mathbf{l}_{A r A}$ and $\mathbf{l}_{A r B}$. This conversion involves the division through the applicable average particle density subsequent to every simulation, as will be described in section 4.3.5. The thus obtained forces per particle are related to the densities and the potentials of mean force of the ArA and ArB particles in the equilibrium systems. See section 4.2.1 for more details. 


\subsubsection{Locations and Spatial Resolution of the Local Observables}

The locations and spatial resolution of the local observables had to be chosen consistent with the simulation length so as to catch the spatial variation of the system in consideration and to obtain reliable data. In BD NEMD simulations, the unphysical system behaviour at the boundary regions had to be additionally taken into consideration in the choice. We shall explain it here using the simulations of the first Ar1Ar2 systems,

E-Ar1Ar2-0.6-1.0-3346-3524-4.74x8.00x9.40-120-ber,

N-Ar1Ar2-0.6-1.0-6795-24-4.74x8.00x9.40-100-140,

N-Ar1Ar2-0.6-1.0-20-7091-4.74x8.00x9.40-100-140, and N-Ar1Ar2-0.6-1.0-3346-3524-4.74x8.00x9.40-100-140,

as examples. This choice was kept for all subsequent simulations. We shall begin with the local observables associated with subvolumes and then come to the stresses which relate to cut-surfaces.

\section{Local Observables Associated with Subvolumes}

Equilibrium interfacial systems are typically divided into slabs parallel to the interface, i.e. perpendicular to the $z$-axis in our systems, for the computation of local observables. One thus takes advantage of the systems' homogeneity parallel to the interface and obtains relatively large subvolumes. Concurrently, the variations perpendicular to the interface can still be determined with a high resolution. In Figure 17, we show exemplarily the distributions of selected local observables and of their standard deviations. These data were obtained by using different numbers, $n_{z}$, of such slabs in E-Ar1Ar2-0.6-1.0-3346-3524-4.74x8.00x9.40-120-ber. Note that the data in the figure were determined only over the first five million time steps of the simulation to save computational effort. As expected in section 4.2.1, the partial densities increase sigmoidally from the phase where the related particles constitute the minority component to the phase where they constitute the majority component. Moreover, the overall densities, which are simply the sum of the partial densities, exhibit a "vacuous gap" in each interfacial region. Finally, the local temperatures are homogeneously distributed throughout the interfacial system, as required for a "field property". In this example we consider a value of $n_{z}=288$ to be the best compromise between a high spatial resolution of the local observables and the reliability of their values. Thus, both minima of the overall densities in the interfacial regions, for instance, are caught well while the noise remains 
acceptable. It becomes particularly apparent away from the interfaces where the system is approximately homogeneous in $z$-direction. Each slab has a $z$-dimension of $L_{z} / n_{z}=0.0326 \mathrm{~nm}$ and is on the average populated by approximately 23 particles: a particle number at the lower limit down to which meaningful values of simple static thermodynamic observables can be determined in EMD simulations in practice [lustig1994a], [lustig1994b], [lustig1994c].

Similar to the equilibrium interfacial systems above, we divided our nonequilibrium systems according to their homogeneities. In doing so, we strived for comparable average subvolume populations. Before a suitable division could be explored, however, it had to be known how rapid the unphysical system behaviour introduced by the thermostating digresses away from the thermostated regions. This digression is particularly hard to determine in the nonequilibrium interfacial systems since their heterogeneity requires relatively large subvolume dimensions in all directions. Instead we studied the digression using one of the first Ar1A2 nonequilibrium one-phase systems, N-Ar1Ar2-0.6-1.0-6795-24-4.74x8.00x9.40-100-140, as a representative example. They allow, similar to the equilibrium interfacial system above, for the determination of the local observables at a high spatial resolution. In the studies, the system was divided according to its homogeneity into $n_{y}=288$ slabs perpendicular to the $y$-axis this time. Figure 18 shows the thus obtained distributions of selected local observables and their standard deviations. They were determined over a simulation length of $75 \mathrm{~ns}$ or $15 \cdot 10^{6}$ time steps. The unphysical system behaviour due to the thermostating manifests itself particularly in the distribution of the temperatures (see Figure 18b). They vary, for instance, much stronger at the thermostated regions than expected from Fourier's law [fourier1822], the variation being slightly more pronounced at the cold thermostated region. BD NEMD simulations in the literature exhibit, even though some of them use different thermostating algorithms, such "temperature jumps" as well [tenenbaum1982], [ikeshoji1994]. The authors ascribed the unlike occurrence of these jumps at the thermostated regions to the different densities there. Since the extension of the jumps is larger at the cold thermostated region while their magnitude is larger at the hot thermostated region, the temperature at the centre between the boundary regions must lie below that of the expected temperature profile, in our systems about $0.4 \mathrm{~K}$ below the expected $120 \mathrm{~K}$ at $|y|=2.0 \mathrm{~nm}$. Apart from the temperatures, the unphysical system behaviour due to the thermostating appears also in the density distribution as a layering of the particles at the thermostated regions (see Figure 18a). Away from them all distributions tend to become linear, which is the natural variation in our nonequilibrium one-phase systems for small temperature gradients (see also section 4.2.2). Based on this tendency, we shall assume conservatively that the unphysical system behaviour due to the thermostating is negligible beyond 
distances of about $1 \mathrm{~nm}$ from the centres of the thermostated regions. Nevertheless, we must mind in our studies the smaller than expected overall temperature level and temperature $y$-gradients in the intermediate region.

Our nonequilibrium interfacial systems have a lower homogeneity than the equilibrium ones or the nonequilibrium one-phase systems: They are only homogeneous along straight lines that lie parallel to the interface and perpendicular to the temperature gradient, i.e. in $x$-direction in our systems. Consequently, we had to come up with a different way of dividing them into subvolumes suitable to catch all characteristic spatial variations. Using the MD simulations of the first nonequilibrium interfacial system, $\mathrm{N}-\operatorname{Ar} 1 \operatorname{Ar} 2-0.6-1.0-3346-3524-4.74 \times 8.00 \times 9.40-100-140$, as an example, we explored by trial and error various such ways of division and found a suitable compromise between spatial resolution and uncertainty of the local observables. Only its outcome shall be given here for the sake of brevity: a subdivision of the system in $y$ - and $z$-direction into a grid of $n_{y}=10$ by $n_{z}=36$ cuboids. They have each a volume of $0.99 \mathrm{~nm}^{3}$ and the dimensions of $L_{x}=4.74 \mathrm{~nm}$ by $L_{y} / n_{y}=0.8 \mathrm{~nm}$ by $L_{z} / n_{z}=0.26 \mathrm{~nm}$. These dimensions lie at the maxima acceptable to catch the spatial variation of the system according to our interests. Thus the $z$-dimension amounts to almost $50 \%$ of the interfacial width of approximately $0.6 \mathrm{~nm}$ (see Tables 11,21, and 30). Its definition is explained in section 4.2.1. The $y$-dimension, on the other hand, is only big enough that the unphysical system behaviour due to the thermostating concentrates on less than half the number of cuboids. Other than that our way of division has the additional advantage that we obtain the observables at the centres between the thermostated regions for comparison to the corresponding equilibrium interfacial systems. Moreover, we shall see in section 5.1.2 that the cuboids are so coarse that the unphysical behaviour in the ones containing parts of the thermostated regions compensates itself in the related results to a large extent. We could therefore consider them as well in our studies, albeit with suspicion.

Due to the chosen coarse resolution, approximately 19 particles can be expected to populate each cuboid on the average: a number of the order of magnitude as required for simple static thermodynamic observables in EMD simulations (see above) and as used in evaluations of NEMD simulations by Tenenbaum et. al. [tenenbaum1982] and Hafskjold et al. [hafskjold1993]. We used cuboids of the aforementioned proportions and population for the evaluation of all subsequent nonequilibrium interfacial systems. Depending on their constraints, the dimensions of the cuboids had to be somewhat adjusted to maintain the above-mentioned average population. The same cuboids were also applied in the other types of systems when the local data is to serve as reference for the nonequilibrium interfacial systems. Other than that we used slabs as in the equilibrium 
interfacial system above. They are oriented perpendicular to the $y$-axis in the nonequilibrium onephase systems. Regardless of the orientation, the thickness of the slabs was always adjusted in such a way that they are populated by approximately 23 particles.

\section{Local Stresses}

Computing the local stresses in a system can easily become expensive, depending on how many exploitable homogeneities exist. We shall explain it here at first in the relatively simple cases of the equilibrium interfacial and nonequilibrium one-phase systems. They are each homogeneous in two dimensions. We then simply obtained the stress distribution along the third dimension, $\alpha$, where $\alpha=z$ in the equilibrium interfacial and $\alpha=y$ in the nonequilibrium one-phase systems, by positioning $n_{\alpha}$ imaginary cross-sectional cut-surfaces perpendicular to it. They had equal distances of $\Delta \alpha=L_{\alpha} / n_{\alpha}$, beginning at the applicable periodic boundaries. Per definition, the motion of every particle within a distance of $\delta \alpha$ from a cut-surface (kinetic contribution) and all pair interactions across it (interatomic force contribution) contribute to its stresses (see section 4.3.2). Since there are no shear stresses, we computed only the normal ones. Equations (4-22)-(4-24) suggest themselves for the computation, since they all apply to cut-surfaces of the same orientation.

As in the case of the volume related observables above, a suitable spatial resolution of the stresses had to be found. Since they relate, however, to cut-surfaces, we can position them close to each other to catch also abrupt variations. Such a positioning comes, of course, with more computations and thus longer simulation run-times, the larger the spatial resolution is. We tried out different values of $n_{\alpha}$ and $\delta \alpha$ in another series of EMD simulations. Without going into their details, $n_{\alpha}=101$ cut-surfaces and a distance of $\delta \alpha=0.1 \mathrm{~nm}$ turned out appropriate for our purposes. These lengths were also used in all subsequent computations of the stresses.

There is no such general homogeneity in the nonequilibrium interfacial systems as in the equilibrium interfacial and nonequilibrium one-phase systems above. Only the normal stresses perpendicular to the $z$-axis, $S_{z z}$, exhibit an approximately homogeneous distribution in $x y$-planes (see section 5.5.2). We took advantage of it to compute them also in the nonequilibrium interfacial systems in the described way. All other normal stresses vary clearly in $y$-and $z$-direction. Moreover, spatially variable shear stresses occur in agreement with the flows. We determined these variations by using confined cut-surfaces of a certain width. Such a confinement produces, of course, more 
noise in the results. We reduced it, however, again somewhat by orienting the cut-surfaces in different directions so as to be able to compute the stresses to their original definitions (see equations (2-5) and (4-21). They are computationally better-behaved than the previous ones rewritten with respect to a single orientation (see e.g. equations (4-22)-(4-24)).

Using the nonequilibrium interfacial system, N-Ar5Ar5-0.6-1.0-3346-3524-4.74x8.00x9.40-100140, as an example, we ran several simulations to find out suitable widths and locations for the confined cut-surfaces. They allow us to determine the local stresses in the interfacial regions. This includes in particular the number of equal cut-surfaces, $n_{\alpha}=101$, in a certain direction and the distance of $\delta \alpha=0.1 \mathrm{~nm}$. For the sake of brevity, we shall present here only the results of the study. Thus, the cut-surfaces perpendicular to the $z$-axis have a width of $1 \mathrm{~nm}$. They are positioned at the centres between integer nanometres along the $y$-axis, e.g. at $y= \pm 0.5, \pm 1.5, \pm 2.5, \ldots \mathrm{nm}$. The cutsurfaces perpendicular to the $y$-axis are either positioned at the $z$-coordinates of the Gibbs dividing surfaces or right in between them into the phases. We make the phasic cut-surfaces just so wide that they range from one interfacial region to the other one. Catching the spatial variation of an interfacial region, however, requires more difficult measures. We divided its cross-sectional area into tenths and considered the 2, 4, 6, 8, and 10 centremost ones of them in the computation of the stresses. Then, the ones on both $n$-nearest tenths to a Gibbs dividing surface are simply the force on the $2 n$ centremost tenths minus the one on the $2 n-2$ centremost tenths and subsequent division of the difference by two tenths of the width of the interfacial region. We thus needed to simulate only five overlapping cut-surfaces instead of ten neighbouring ones and still obtain the same resolution. Asymmetries constitute no problem, since we applied the above-mentioned procedure only in homophasic nonequilibrium interfacial systems. Figure 19 highlights selected cut-surfaces to illustrate again their different locations and orientations. They were used similarly in other systems but we studied only selected ones due to the computational expense connected with the determination of the local stresses. 


\subsubsection{Summarisation of the Local Data}

Subsequent to each simulation, we converted the interatomic volume forces, $\mathbf{i}_{A r A}$ and $\mathbf{i}_{A r B}$, which were defined in section 4.3.3, into interatomic forces per particle, $\mathbf{t}_{A r A}$ and $\mathbf{t}_{A r B}$, as used in section 4.2.1, by division through the applicable partial densities:

$\mathbf{\mathbf { v }}_{\mathrm{ArA}}=\frac{\mu\left(\mathbf{i}_{A r A}\right)}{\mu\left(\rho_{A r A}\right)}$

$\mathbf{t}_{A r B}=\frac{\mu\left(\mathbf{i}_{A r B}\right)}{\mu\left(\rho_{A r B}\right)}$.

In principle, we could have determined these mean forces as well on-the fly during the simulations. However, the minority component does not always populate every subvolume in a phase. Although we computed the mean forces only subsequent to every simulation, they agree quite well with the expected ones from the BGY equation, as we shall see in section 5.1.1. The standard deviations of the mean forces per particle were not computed.

After the conversion of the forces above, we averaged the local data of the local observables in order to ease the comparison of the different systems. When dealing with an interfacial one, however, we additionally relocated the local data in two ways prior to the summarisation. In the relocation, we shifted first of all the distributions, including the ones of the normal stresses, in $z$ direction. These shifts were conducted by integer multiples of the subvolume dimension in this direction. Concurrently, we sticked to the convention that the phase dominated by the particles mentioned first in the notation of the underlying system is distributed as symmetrically as possible about the $x y$-plane. Full symmetry cannot be achieved, however, because the distributions themselves are slightly asymmetric regardless of the noise. This asymmetry results from the coarse spatial resolution in $z$-direction together with the different arrangements of the subvolumes in both interfacial regions with respect to their spatial variation. In particular, the extreme values of some observables can deviate significantly in the interfacial regions as e.g. in the case of the overall densities (see Figure 35). In order to further enhance the comparability of the distributions of a system, we reflected all its local data about the $x y$-plane if the lower interfacial minimum of the overall densities is not located at negative $z$-coordinates. 
Subsequent to the force conversion, and possibly the relocation, above, the distributions of the local observables were averaged. In this way, we reduced, on the one hand, redundant data. Such redundancies result from the symmetries and homogeneities in our systems. Thus, the normal stresses lateral to the cross-sectional homogeneities of our systems, e.g. $S_{x x}$ and $S_{y y}$ in our equilibrium interfacial ones, were averaged into single values represented by the symbol $S_{x x y y}$. Moreover, spatial averaging of the local observables was possible. We shall explain it in the subsections below for the various types of the systems. Later, we also simulated, as we call them, homophasic interfacial systems. Their substances have identical interaction parameters so that both phases exhibit the same properties. We then additionally averaged the related observables in both phases. In this averaging, particle specific observables, such as the partial densities for instance, were given a new subscript, depending on whether they relate to the applicable majority or minority component in a phase. It sometimes made sense to average also in systems with higher order symmetries or homogeneities the local com velocities at common $z$ - and absolute $y$-coordinates only. Such averaging simplifies comparisons to the nonequilibrium interfacial system. Apart from the reduction of the data, the averaging generally also leads to more reliable numerical values. Hence, we averaged the volume and particle forces, which are subject to a particularly strong noise (see section 4.2.2), over all $y$-coordinates in the investigation regions of our nonequilibrium systems. The obtained results, we shall call them I-averages, apply approximately at the centres between the thermostated regions.

In order to make clear for every observable if and over what region it has been spatially averaged, we shall introduce a special notation. It consists of the symbol $\varnothing$ followed by the symbol of the observable, a colon, and the designation of the region, over which the spatial average was taken, in brackets. The average of the com $z$-velocities at common $z$ - and absolute $y$-coordinates, would for instance be denoted as $\varnothing\left[\mu\left(v_{z}\right):|y|, z\right]$. Similarly, the spatial average of the Ar1 partial density standard deviations in all subvolumes of a given system would, for example, be denoted as $\varnothing\left[\sigma\left(\rho_{A r l}\right): S\right]$. Here, the letter $S$ states that the entire system is considered in the averaging. Finally, the average $\varnothing\left[\sigma\left(\rho_{M a j}\right): \mathrm{P}\right]$ is over the partial densities of the locally applicable majority component in both phases of a homophasic equilibrium interfacial system. Further designations of the regions averaged will be introduced as part of the explanations below. Moreover, we shall give details on the computation of the additional properties that are derived from the averaged data to help characterise the distributions of the local observables. 


\section{Equilibrium Interfacial Systems}

We stated already in section 4.1.1 that the equilibrium interfacial systems are homogeneous in $y$ direction and symmetric with respect to the $x y$-plane. This symmetry manifests itself, however, only approximately in the distributions of the local observables, as explained above. We therefore averaged solely the data at common $z$-coordinates. Additional averaging is possible away from the interfaces where the systems tend to become also homogeneous in $z$-direction. We generally assumed homogeneity beyond a certain, to be defined below, distance away from the interfaces. As a result, the thus demarcated subvolumes could be lumped together into "phases" (see Figure 20). In particular, we spatially averaged the different local observables within each phase. We shall use the symbol $\varnothing$ introduced above, followed by the observable in consideration and the name of the majority component of the applicable phase in brackets, to identify such phase or, in brief, Paverages. Thus, the ones of the Ar1 particle density in the Ar2 phase would, for instance, carry the symbol $\varnothing\left[\mu\left(\rho_{A r 1}\right): A r 2\right]$. In chapter $\mathbf{5 . 3}$ we shall compare hetero- and homophasic interfacial systems. This requires also separate averages over the local observables in each phase of the homophasic systems. In order to distinguish these averages, those in a phase rich in the first component shall have its name followed by the encircled number with a white background, $\varnothing[\mu(\rho): \operatorname{Ar} 5]$. Averages in the other phase append the name of the second component followed by the encircled number with a black background.

Our systems will turn out to behave mostly according to our expectations from section 4.1.1. Particularly, the density distributions are closely approximated by the hyperbolic tangent functions (see e.g. section 5.1.1). They thus provide measures for the widths and $z$-locations of the interfaces. We computed these properties as an additional part of the simulation results to ease their understanding. To be more exact, four different widths and four $z$-locations are obtained. We distinguish them by complementing their symbols by the subscripts "+" or "-", depending on whether the related interface is located at positive or negative $z$-coordinates. Moreover, we computed averages of the four interfacial widths,

$$
\omega=\frac{\omega_{A r A-}+\omega_{A r B-}+\omega_{A r A+}+\omega_{A r B+}}{4},
$$

as well as of the two $z$-locations in each interfacial region, 


$$
\zeta_{-}=\frac{\zeta_{A r A-}+\zeta_{A r B-}}{2} \text { or } \zeta_{+}=\frac{\zeta_{A r A+}+\zeta_{A r B+}}{2}
$$

These averages were also used to demarcate the "phases" in our systems as stated above. We considered all subvolumes beyond a distance of two times the average interfacial width from the average $z$-locations of the Gibbs dividing surfaces as part of the phases. The Hyperbolic tangent density profiles generally approximate their nearest limits by more than $99.9 \%$ at such a distance. It is also sufficient for our purposes since all four interfacial widths almost agree in each simulation, as do different $z$-locations for each interface (see Tables 10 and 20 as well as Tables 11, 21, and 30).

In addition to the widths and $z$-locations of the interfaces, their tensions were computed by numerical integration, as a supplement to the simulation results. In the computation, we averaged both normal stresses lateral to the interface by applying equation (4-1) correspondingly to our systems. Although its integral runs between the periodic boundaries only, i.e. from $-L_{z} / 2$ to $+L_{z} / 2$, we can neglect the thus resulting error because the systems are large enough for the phases to assume a bulk-like behaviour (see section 5.1.1). In order to obtain the interfacial tension, the value of the integral had to be halved due to its inclusion of two interfacial regions. Finally, we computed the potentials of mean force according to equations (4-6) and (4-7).

\section{Nonequilibrium One-Phase Systems}

The nonequilibrium one-phase systems are fully homogeneous in $z$-direction and symmetrical with respect to the $x y$-plane, as stated in section 4.2.2. Hence, we averaged the values of the local observables at common absolute $y$-coordinates. Moreover, we computed the I-averages of the interatomic $y$-forces per unit volume or per particle over all $z$-coordinates in the investigation regions as stated above. Such averages shall be designated by the symbol $\varnothing$ followed by the observable in consideration, the name of the majority component in the phase, and the absolute $y$ coordinate, in nanometres, of the associated subvolumes in brackets. For simplicity's sake, we hall also denote the averages at selected $y$-coordinates as $\mathrm{H}-, \mathrm{C}-$, and $\mathrm{M}$-averages: The "H-" and "Caverages" are averages over those subvolumes that reach into either the hot or the cold thermostated region, whereas the "M-average" considers the subvolumes with $y$-coordinates in the middle between both regions. The absolute $y$-coordinates are alternatively replaced by the letters $\mathrm{H}, \mathrm{C}, \mathrm{M}$, or I in the identification of these averages. Thus, the M-average of the Ar1 particle density in the Ar2 phase would, for instance, carry the symbols $\varnothing\left[\mu\left(\rho_{A r 1}\right): A r 2,|y|=2.0\right]$ or $\varnothing\left[\mu\left(\rho_{A r 1}\right): A r 2, M\right]$. 
The distributions of the local observables obtained in the simulations (see section 5.1.2) will prove to agree with our expectations from section 4.2.2. In order to help grasp the distributions of the local observables, we derived additional properties from the averaged data. Thus, $y$-gradients of the temperatures and densities were computed by linear regression of the averaged data. In our computations we had to mind the unphysical system behaviour due to the thermostating (see section 4.3.4). Accordingly, only the data with $y$-coordinates beyond a distance of $1 \mathrm{~nm}$ from the centres of the thermostated regions were considered. Finally, we determined also the Soret coefficients according to equation (4-8), where the substance ArA is chosen to be the first mentioned one in our denomination of the system considered and the substance ArB is to be the second-mentioned substance. Since the mole fractions, $x_{A r A}$ and $x_{A r B}$, must always add up to one in a binary system, we shall give the Soret coefficients only with respect to the first mentioned substance. It won't be mentioned again in the subscript. The values of the mole fractions, $x_{A r A, O}$ and $x_{A r B, O}$, were obtained from the applicable $M$-averages, i.e. the densities at $|y|=2.0 \mathrm{~nm}$. In systems with an even number of $y$-coordinates, such as N-Ar5Ar5-0.6-1.0-13384-14096-4.74x16.0x18.8-110-130 for instance, we computed the $\mathrm{M}$-averages by averaging the values at the coordinates that lie closest to the centres between the thermostated regions. Comparisons between corresponding EMD and NEMD simulations will also use these $\mathrm{M}$-averages.

\section{Nonequilibrium Interfacial Systems}

The symmetry of the nonequilibrium interfacial systems with respect to the $x y$-plane appears only approximately in the distributions of the local observables, as explained for the equilibrium interfacial system above. Hence, we generally averaged only the data at common $z$ - and absolute $y$ coordinates. However, the thermocapillary convection will turn out weak so that our systems tend to become homogeneous in $z$-direction away from the interfaces (see e.g. section 5.1.3). As a result of this tendency, we could determine the widths and locations of the interfaces at selected $y$ coordinates, as described for the equilibrium interfacial system above. Moreover, we could assume homogeneity beyond a subsequently defined distance from the interfaces and lumped the thus demarcated subvolumes together into phases (see Figure 21). Each phase could then be considered as a separate nonequilibrium one-phase system. Thus, we computed its abovementioned spatial averages and the derivational properties defined for it. The name of the phase, that they relate to, is simply appended to their designation after a colon, e.g. $S_{T}$ :Ar1 in an Ar1 phase. In order to further characterise the interfacial regions, we considered the nonequilibrium interfacial system as 
composed of different equilibrium ones. Thus, their abovementioned derivational properties were computed at every $y$-coordinate, if possible. We append it as well to the designations of these properties in brackets.

Our nonequilibrium interfacial systems will prove to behave mostly as expected for their weak thermocapillary convection. Thus, we can fit the hyperbolic tangent profiles surprisingly well on the density distributions at any $y$-coordinate (see e.g. section 5.1.3). The dividing surfaces in these profiles hardly shift in $z$-direction when going from one $y$ coordinate to another one. A somewhat more distinct $y$-dependence exists, however, in the case of the interfacial variation of these profiles. Its width differs up to $25 \%$ from the one at the centres between the thermostated regions, i.e. $|y|=2.0 \mathrm{~nm}$. Nevertheless, such a $y$-dependence is small enough to safely demarcate the "phases" as in an equilibrium interfacial system from above. Hence, we sticked with this demarcation also in the nonequilibrium interfacial systems. For simplicity's sake, however, the widths and $z$-locations at the centres between the thermostated regions were also used in the demarcation at any other $y$ coordinate. 


\subsection{Preparational and Production Simulations}

As mentioned in part 3, we distinguish preparational and production simulations. In the preparational simulations, the systems are gradually eased toward a state compatible with the specified constraints. This easing starts typically from a non-representative, but therefor easy to provide, initial phase point of that system. Depending on whether the system assumes a thermodynamic quilibrium or a stationary nonequilibrium state, we further distinguish the "preparational simulations" into "equilibration simulations" or "steadying simulations". The production simulations start from a so-prepared state and compute, in one or several batches, an arbitrarily long, he, arbitrarily long representative section of the phase space trajectory. Since the preparational runs are discarded for the actual analysis of the system behaviour, "anything goes" in them, as well as in the generation of their initial phase points, as long as representative phase points are produced towards the end. To economise the preparational simulations, however, initial phase points as close as possible to the wanted final ones should be used.

Our systems (see Figure 22) differ from each other only in individual parameters, such as e.g. the particle mass or the dimensions. We therefore reused suitable final phase points of earlier production simulations - after minor alterations if necessary - as starting points for preparational simulations of similar systems. However, several initial phase points obtained through different preparation routes were used for the very first nonequilibrium interfacial system: an Ar1Ar2 mixture under the default constraints. In this way we checked whether, and how far, the backstory of such a system affects its behaviour. Section 4.4.3 explains in detail the preparation of these initial phase points together with the subsequent production runs. In advance of the first nonequilibrium interfacial system, we simulated its corresponding equilibrium interfacial system and nonequilibrium one-phase systems to check the suitability of our simulation code and to have a as a reference. For clarity's sake, these simulations shall be explained separately here according to the different types of the systems. In the order of their complexity, we look at the preparational and production simulations of the equilibrium interfacial system first (see section 4.4.1) and afterwards at the ones of the corresponding nonequilibrium one-phase systems (see section 4.4.2). They reproduce the phases observed in the equilibrium interfacial system separately as subjects to the default dimensions and target temperatures of the nonequilibrium interfacial system. In the explanations below, the simulations shall be generally named after the system. In the case of the 
preparational simulations, the first letters " $\mathrm{N}$ " or "E" are written in lower-case "n" or "e", e.g. nAr1Ar2-0.6-1.0-3346-3524-4.74x8.00x9.40-100-140. Section 4.4.4 addresses the other preparational and production simulations of the later systems.

All preparational and production simulations, conducted in the course of this work, use a time step of $5 \mathrm{fs}$ for the numerical integration of the equations of motion. This step size has proven to be a good compromise between computational expenses and numerical accuracy in previous EMD simulations of mixtures comparable to ours [buhn2004a], [buhn2004b], [buhn2006] using a similar integration algorithm, viz. the Verlet-Leapfrog method [hockney1988]. 


\subsubsection{First Equilibrium Interfacial System}

First of all, we checked the accuracy of our simulation code in comparison to the original DL_POLY_2 Molecular Simulation Package (see section 4.5) by conducting different EMD production runs of the first equilibrium interfacial system:

- one under the default constraints, E-Ar1Ar2-0.6-1.0-3346-3524-4.74x8.00x9.40-120-ber, using our simulation code with a Berendsen thermostat, as explained in section 4.1,

- one under the same constraints, E-Ar1Ar2-0.6-1.0-3346-3524-4.74x8.00x9.40-120-nht, using the original DL_POLY_2 Molecular Simulation Package with a Nosé-Hoover thermostat [nose1984], [hoover1985] (time constant $t_{T}=1.25 \mathrm{ps}$ ),

- one under NVE constraints, E-Ar1Ar2-0.6-1.0-3346-3524-4.74x8.00x9.40--71.5, using our simulation code, where the particle numbers and system dimensions have the default values and the value of the system energy was inherited from the final phase point of the preceding equilibration.

All of the simulations above are $75 \mathrm{~ns}\left(15 \cdot 10^{6}\right.$ ts $)$ long, have equivalent constraints, and start from the same initial phase point so that deviations in the results can be ascribed to the implementation. We shall describe further below the preceding equilibration simulations and how we ensured in them that this phase point is representative. The last two production runs can be considered as physically more sound because they generate states in the canonical or microcanonical ensemble. We apply the following notation for the three simulations above to ease their distinction. Thus, the $N V T$ ones of them are complemented by the abbreviation "ber" for Berendsen thermostat or "nht" for the NoséHoover thermostat, where applicable. The $N V E$ simulation has the system energy "-71.5" instead of the temperature constraint in the notation.

In the comparison, however, no distortions of the system behaviour relevant to our studies could be found. Rather, the results are very similar in all three simulations. Thus, both the global temperatures and pressures, which, according to Gibbs's phase rule (see section 4.2.1), specify unambiguously the thermodynamic state of the underlying equilibrium interfacial system with given particle numbers, differ less than $4 \%$, see Table 14. It might appear surprising at first sight that the pressures and temperatures in the NVE simulation are distinctly smaller than in the other ones. We must, however, keep in mind that we inherited an "unfavourable" value for the energy constraint, 
$E=-71.5 \mathrm{aJ}$, from the final phase point of the preceding equilibration simulation, e-Ar1Ar2-0.6-1.03346-3524-4.74x8.00x9.40-120-nht, with an average energy of $\mu(E)=-71.3 \mathrm{aJ}$. The pressure and temperature difference could therefore be further reduced by adjusting the energy constraint to this average value, which approximately also continued to be observed in the other production runs. The mentioned energy averages can be found as well in Tables 14, 24, and 33.

In addition to the global observables also the distributions of the local observables strongly resemble each other in the different simulations (see Figure 23). Thus, the densities, for instance, deviate by less than $2 \%$ (see Figure 23a). Relatively large deviations occur in the interfacial regions where the spatial resolution is too low to catch the variations entirely. Since these deviations are approximately antisymmetric about the origin, we ascribe them to a different arrangement of the simulated matter with respect to the subvolumes in the three production runs. For the same reason, particularly the minima in both interfacial regions slightly differ even within the same run. We must bear in mind, however, that a higher spatial resolution is not achievable in the determination of the local observables, as explained in section 4.3.4. Note also that the distributions obtained from the production simulation using the original DL_POLY_2 Molecular Simulation Package with a NoséHoover thermostat bear relatively strong noise. To make it short we don't give any results here, but we observed the same phenomenon when comparing NVE simulations using the original code to ones under the same constraints using our simulation code. Since both codes strongly resemble each other otherwise, we ascribe the stronger noise to the application of the Verlet-Leapfrog integration algorithm [hockney1988] in the original code, whereas we use the numerically more accurate Velocity-Verlet algorithm [swope1982]. Other than that, there are no recognisable systematic deviations between the simulations.

Instead of comparing the results of the different simulations directly, the accuracy of our simulation code can also be assessed using the deviations of selected local observables, that must be homogeneously distributed throughout a system, from their systemwide values. In particular the rescaling of the particle velocities as part of the thermostating can generally induce translational or rotational collective motions of the particles [harvey1998], [leyssale2008] in MD simulations. As stated in section 4.1.4, we took great care to avoid such motions, when implementing our thermostating algorithm, because they interfere with the expected thermocapillary convection in our nonequilibrium interfacial systems. Hence, the EMD simulation E-Ar1Ar2-0.6-1.0-3346-3524$4.74 \times 8.00 \times 9.40-120$-ber can count as a preliminary test of our algorithm, when used to implement a Berendsen thermostat. In the next section, we shall test the algorithm also in NEMD simulations. 
We reset the system com velocity to zero during the preparational simulations. The local com velocities (see Figure 23c) are, s as in the other simulations, homogeneously distributed on this level with standard deviations $\varnothing\left[\sigma_{s}\left(v_{y}\right): S\right]$ and $\varnothing\left[\sigma_{s}\left(v_{z}\right): S\right]$ of less than $0.18 \mathrm{~m} / \mathrm{s}$, which supports the suitability of our code for our studies. Also the local temperatures (see Figure 23b), which serve as another criterion, deviate by less than $0.5 \%$ from their global value in each simulation.

Since the system behaviour in the three production runs is thus essentially equivalent, apart from some minor deviations, we regard our simulation code as accurate in terms of our EMD simulations. For convenience, we shall consider henceforth only the production run that uses a Berendsen thermostat, E-Ar1Ar2-0.6-1.0-3346-3524-4.74x8.00x9.40-120-ber, in our studies. This simulation delivers also the initial phase point for other EMD and NEMD simulations to come (see Figure 22).

We obtained the initial phase point used in the production runs above from an equilibration simulation of 30ns under the default constraints (e-Ar1Ar2-0.6-1.0-3346-3524-4.74x 8.00x9.40-120nht). It was run by means of the original DL_POLY Molecular Simulation Package. The equilibration itself started from a mixed crystalline arrangement of the Ar1 and Ar2 particles in simple interpenetrating orthorhombic Ar1 and Ar2 lattices (see Figure 24). The particle velocities were randomly assigned according to their probability in the Maxwell-Boltzmann velocity distribution function at $120 \mathrm{~K}$. A Nosé-Hoover thermostat with a time constant of $t_{T}=1.25 \mathrm{ps}$ [nose1984], [hoover1985] maintained the system at the intended temperature. We observed the progress of the equilibration by monitoring the chronological evolution of the instantaneous local particle densities. The system behaviour, shown as snapshots at different simulation times in Figure 25, is exactly as expected in section 4.1.1: The initially randomly positioned particles migrate to form heterogeneous islands which in due course aggregate into two phases, with the two (due to the system symmetry) smallest-possible interfaces perpendicular to the $z$-axis. Once the plane interfaces are established, after about $2 \mathrm{~ns}$, the system remains stationary for the remainder of the equilibration simulation. We verified the stationary state of the system by averaging the instantaneous local observables over three 10ns long successive segments of the computed phase space trajectory. Thus, the densities and temperatures from the second segment persisted also in the third one, except for minor deviations (see Figure 26). We therefore assume the latest phase points of the simulation to be representative for an equilibrium interfacial system. The final phase point is then used as the initial phase point for the EMD production runs above and the NEMD steadying simulations of the first nonequilibrium interfacial system below in section 4.4.3. 


\subsubsection{First Nonequilibrium One-Phase Systems}

We established in the previous section that our thermostating algorithm induces no convection - at least when used to implement a Berendsen thermostat. However, it still remains to be seen if the same holds also when the algorithm is used for its original purpose: to establish and maintain a temperature gradient in NEMD simulations. In order to check this, we reproduced the phases observed in the equilibrium interfacial system, E-Ar1Ar2-0.6-1.0-3346-3524-4.74x8.00x9.40-120ber, separately in nonequilibrium one-phase systems (N-Ar1Ar2-0.6-1.0-6795-24-4.74x8.00x9.40100-140 and N-Ar1Ar2-0.6-1.0-20-7091-4.74x8.00x9.40-100-140) subject to the same dimensions and temperature constraints as the nonequilibrium interfacial system. The particle numbers were simply obtained by multiplying the density phase averages, given in Table 8, with the system volume. Figure 34e,f shows the com $y$ - and $z$-velocity distributions obtained in the NEMD production runs of $75 \mathrm{~ns}\left(15 \cdot 10^{6} \mathrm{ts}\right)$ by means of our simulation code. No convection can be recognised in these distributions. Instead they are approximately homogeneous on a level with the system com velocity. It was set to zero during the preparational simulations. They will be explained further below. The standard deviations of the com velocity components $\varnothing\left[\sigma_{s}\left(v_{y}\right): S\right]$ and $\varnothing\left[\sigma_{s}\left(v_{z}\right): S\right]$ amount to less than $0.18 \mathrm{~m} / \mathrm{s}$, as in the equilibrium interfacial systems from the previous section. We can therefore assume that our thermostating algorithm qualifies also for our NEMD simulations and that the convection observed in the nonequilibrium interfacial systems has a natural cause. More aspects in the behaviour of the nonequilibrium one-phase systems will be discussed later in section

\subsection{2.}

For the sake of completeness, we briefly explain the preparational simulations here that preceded the production runs. The initial phase points for these preparational simulations were simply generated by renaming the particles in the final phase point of e-Ar1Ar2-0.6-1.0-3346-35244.74x8.00x9.40-120-nht and by introducing the still missing particles at random positions until the desired particle numbers in the systems are reached. All particle velocities were randomly reassigned according to their probability in the Maxwell-Boltzmann velocity distribution function at 120K. Then, we conducted NEMD steadying simulations of $30 \mathrm{~ns}\left(6 \cdot 10^{6} \mathrm{ts}\right)$ by means of our simulation code (n-Ar1Ar2-0.6-1.0-6795-24-4.74x8.00x9.40-100-140 and n-Ar1Ar2-0.6-1.0-207091-4.74x8.00x9.40-100-140). The strict thermostats provided by it established the default temperature difference of $40 \mathrm{~K}$. To avoid arithmetic overflows, we capped the particle forces by 
using the corresponding DL_POLY function during the first 50ps $\left(1 \cdot 10^{4}\right.$ time steps). The evolution of the systems towards a stationary nonequilibrium state was observed by monitoring the local particle densities and temperatures. We determined them over three successive thirds of the computed phase space trajectories. Linear temperature and density distributions were established already in the first third between the thermostated regions. Since these distributions persisted, apart from minor fluctuations, in the successive segments, we assumed that the final phase points of the steadying simulations are suitable for the production runs. Figure 27 shows exemplarily the densities and temperatures determined over the different thirds in n-Ar1-0.6-1.0-6795-24$4.74 \times 8.00 \times 9.40-100-140$. 


\subsubsection{First Nonequilibrium Interfacial System}

As stated in section 3.2, a necessary condition for the representativeness of MD simulations is the independence of the observables from the initial phase point. We checked this condition explicitly by using our NEMD simulations of the first nonequilibrium interfacial system, an Ar1Ar2 mixture under the default constraints (N-Ar1Ar2-0.6-1.0-3346-3524-4.74x8.00x9.40-100-140), as an example. This system was simulated in three separate production runs of $75 \mathrm{~ns}\left(15 \cdot 10^{6}\right.$ time steps). They all use the same simulation code with the strict thermostats (see section 4.1.4). Only the initial phase points, which were constructed through different routes. Each such route involves preparational simulations either run under different "system constraints" or starting from a completely different phase point (see Figure 24). The subsections below describe the three routes. In order to avoid confusion, these different simulations shall be named after the system followed by the letter "r" for "route" and its applicable number, e.g. N-Ar1Ar2-0.6-1.0-3346-3524$4.74 \times 8.00 \times 9.40-100-140-r 1$.

Table 14 shows the values of the global observables in all three production runs. The values strongly resemble each other, i.e. with differences of less than $0.25 \%$, except for the heat fluxes. We shall see in section 5.2.3 that their considerable fluctuations, i.e. with differences up to $15 \%$, result from the "strict thermostats" applied. The distributions of the local observables also strongly resemble each other. For the sake of brevity, we compare only selected distributions as examples here. More detailed discussions will follow later in part 5. As stated already for the equilibrium interfacial system in section 4.4.1, the deviations contain errors due to the different arrangements of the simulated matter with respect to the subvolumes in the production runs. These errors manifest themselves particularly in symmetries of the deviations about the origin, e.g. in the case of the densities (see Figure 28a). Nevertheless, their local values differ by less than $2 \%$ between the simulations. The temperatures (see Figure $28 \mathrm{~b}$ ) deviate by less than $0.3 \%$ and the local velocity components (see Figure 28c) by less than $0.4 \mathrm{~m} / \mathrm{s}$ for a value range between approximately $-2 \mathrm{~m} / \mathrm{s}$ and $1 \mathrm{~m} / \mathrm{s}$. Based on these resemblances under identical system constraints, we shall assume that the system behaviour observed in the three production runs is essentially equivalent. For convenience, we discard two production simulations in the remainder of this work and consider only N-Ar1Ar20.6-1.0-3346-3524-4.74x8.00x9.40-100-140-r1. Instead, we might as well have chosen the 
simulations of the second or third route. The considered simulation delivers also the initial phase point for the simulations of the later nonequilibrium systems (see Figure 22).

\section{First Preparation Route}

For the first preparation route, we drew upon EMD simulations of equilibrium interfacial systems by Buhn et al. [buhn2004a], [buhn2004b]. We borrowed from them the default values of our system constraints, in terms of density and thermostated temperatures (see section 4.1.3), as well as the LJ parameters for the interactions between like Ar1 and Ar2 particles (see section 4.1.2). Slices with the dimensions of $4.74 \mathrm{~nm} \times 8.00 \mathrm{~nm} \times 9.47 \mathrm{~nm}$ and of $4.74 \mathrm{~nm} \times 4.00 \mathrm{~nm} \times 9.47 \mathrm{~nm}$ were cut from the final phase points of the author's NPT simulations. They are each at ambient pressure but at different temperatures of $100 \mathrm{~K}, 108 \mathrm{~K}, 116 \mathrm{~K}, 126 \mathrm{~K}, 132 \mathrm{~K}$, or $138 \mathrm{~K}$. See below for more details. We then joined these slices with each other in $y$-direction in increasing order of their temperatures, as shown in Figure 29, and shifted the momentum of every particle by a constant vector so that the total momentum of all particles amounts to zero. As a result, we obtained an initial phase point for our preparational simulations that accounts already for the expected temperature distribution in our nonequilibrium systems - even though only very crudely.

The dimensions of the slices were chosen as follows. Their $x$-dimension equals the median of the $x$ dimensions, $L_{x}$, in the NPT simulations by Buhn et al. . The errors made by using it rather than the actual values were small and therefore neglected. The $z$-dimension, $L_{z}$, on the other hand, was chosen to be only two-thirds of the median one of these simulations, so that both phases have approximately the same size. Finally, the $y$-dimension, $L_{y}$, was chosen in a way that the resulting total number of particles remains manageable. Figure 29 also indicates the number of particles contained in each slice, resulting in a total of 3346 Ar1 and 3524 Ar2 particles. To make sure that both phases can still develop a bulk-like behaviour, i.e. despite the shorter $z$-dimension, we reduced, through the Lorentz-Berthelot mixing parameter, $\xi$, the miscibility of both species and thus also the interfacial width (see section 4.1.2). Furthermore, we increased the system pressure by reducing the $z$-dimension of the system boundaries to $L_{z}=9.40 \mathrm{~nm}$. These system dimensions and particle numbers were kept, together with the value of the mixing parameter $\xi=0.6$, for the other two routes and constitute the default values for all subsequent simulations (see section 4.1.3). 
The so-constructed phase point was used as starting point for a NEMD steadying simulation of 30ns under the default constraints (n-Ar1Ar2-0.6-1.0-3346-3524-47.4x8.00x94.0-100-140-r1). Particles lying outside the boundaries at the start of the simulation were automatically reinserted by the implementation of the periodic boundaries in DL_POLY. To avoid arithmetic overflows, the interparticle forces were capped by using the applicable DL_POLY routine during the first 500fs. Concurrently, we reset the parameters of thermostating algorithm to $k_{P}=0.1$ and $k_{I}=k_{D}=0$ for this period. As a result, only reduced amounts of the kinetic energy, necessary to establish the desired temperatures in the thermostated regions, were coupled into or out of them after each integration step. We then shifted all particle momenta by a suitable amount to reset the total system momentum and applied the strict thermostats for the remainder of the simulation. The evolution of the system towards its stationary nonequilibrium state was observed by monitoring the local particle densities and temperatures. They were determined over three successive segments of the computed phase space trajectory of $10 \mathrm{~ns}$ each (see Figure 30). Already in the first segment approximately linear density and temperature $y$-profiles are established between the thermostated regions. Since these distributions also persist, apart from minor fluctuations, in the successive segments, we assumed that the last phase points of such simulations are suitable starting points for the production runs of the "nonequilibrium interfacial system".

\section{Second Preparation Route}

We used the initial phase point of the EMD production simulation above also for a NEMD steadying simulation of 30ns. It is under the default constraints (n-Ar1Ar2-0.6-1.0-3346-3524$4.74 \times 8.00 \times 9.40-100-140-r 2$ ) with strict thermostats. We observed the removal of the system from equilibrium in the same way as during the steadying simulation of the first preparation route. As described above, the local temperatures and densities, averaged over the first segment of the computed phase space trajectory, vary linearly between the "thermostated regions" (see Figure 31). The variation persisted when determined over the second and the third segments. We therefore assumed that the steadying simulation had reached a stationary state again. Hence, the last phase point is representative for the "nonequilibrium interfacial system". 


\section{Third Preparation Route}

The initial phase point of the equilibration (e-Ar1Ar2-0.6-1.0-3346-3524-4.74x8.00x9.40-120-nht) discussed above was also used in the third route for a steadying simulation of 30ns under the default constraints (n-Ar1Ar2-0.6-1.0-3346-3524-4.74x8.00x9.40-100-140-r3). To avoid arithmetic overflows, only reduced amounts of the kinetic energy were coupled into or out of the thermostated regions during the first $5 \mathrm{ps}$, similar to the first preparation route above. After this period, we applied the strict thermostats for the remainder of the simulation. As in the corresponding equilibration simulation, we monitored the instantaneous local particle densities to observe the evolution of the system. Thus, the mixture separates again into an Ar1 and an Ar2 rich phase with two flat interfaces perpendicular to the interface. The plane interfaces are established after about 1ns (see Figure 32). Additionally, we averaged the instantaneous local temperatures and particle densities over three successive 10ns long segments of the computed phase space trajectory. Both the temperatures and particle densities vary linearly between the thermostated regions already in the first segment (see Figure 33). As in the other preparation routes before, we assumed that the steadying simulation had converged toward a representative stationary state because the distributions of these observables persisted, when computed over the second and the third segment.

Before we move on to the further simulations in the next section, one remark on the system behaviour in the preparational simulations is in order. The plane interfaces were established more quickly during the steadying simulation of the third preparation route (n-Ar1Ar2-0.6-1.0-33463524-4.74x8.00x9.40-100-140-r3) than during the equilibration simulation (e-Ar1Ar2-0.6-1.03346-3524-4.74x8.00x9.40-120-nht). The different speeds of separation suggest that the phase separation could be promoted by a thermocapillary convection in the steadying simulation. It is, however, unclear, from the particular cases considered, to what extent the preparational simulations are representative for the related nonstationary nonequilibrium systems. Since nonstationary NEMD simulations are still subject of research, we do not follow up on this hypothesis in our studies. 


\subsubsection{Later Systems}

A multitude of systems is simulated in the course of this work. Tables 5, 15, and 25 give an overview of the details. Additional ones are provided, insofar as they become relevant, when we discuss the individual systems. As describe above, the preparational simulation ahead of each production run starts from the final phase point of an earlier run with similar parameters. Figure 22 visualises which parameters are altered and how the runs follow each other. Alterations, that do not involve the total particle number, were simply made by changing the input parameters, e.g. the LJ parameters, the masses, the cut-off radius $r_{c}$, or the system dimensions. We stated already in the previous section that DL_POLY automatically reinserts the particles, that reside outside of the sodefined box, back into the system. For alterations involving the total particle number, several copies of the final phase point are joined to form a new starting point, e.g. in N-Ar5Ar5-0.6-1.0-33463524-4.74x8.00x18.8-100-140. To avoid arithmetic overflows following such expansions, the maximum interparticle forces and the amounts of kinetic energy to add to or withdraw from the thermostated regions must be reduced during the first time steps of the preparational simulation. If necessary, we additionally reset the system momentum to zero by shifting all particle momenta by a suitable amount.

Due to the similarity of the successive production simulations, their intermediate preparational simulations can be shorter than described above. Depending on the "severity" of the alterations we chose lengths ranging from $5 \mathrm{~ns}$ to $20 \mathrm{~ns}$, as shown in Figure 22. The progress of the preparational simulations was monitored, as described previously; for the sake of brevity, we omit the details here. 


\subsection{Simulation Code and Hardware}

There was no ready-made simulation software available for our needs. Hence, we upgraded an existing code according to our needs. The DL_POLY_2 Molecular Simulation Package Version 2.13, developed at Daresbury Laboratory by W. Smith, T.R. Forester and I.T. Todorov [smith2007b] served as a basis. This package provides many of the necessary features such as the data in-/output or the calculation of the particle interactions also known as the "force field".

We implemented the thermostats necessary to deflect and maintain the simulation system out of equilibrium. Section 4.1.4 describes their algorithms in more detail. They work best if the particle positions and momenta are known simultaneously at every time step. The Verlet-Leapfrog integration algorithm [hockney1988] used in the original DL_POLY Simulation Package computes the particle momenta and positions, however, at different times. Hence, we implemented the socalled Veloctiy-Verlet algorithm [swope1982]. Both algorithms are compared e.g. in [allen2003]. Finally, we implemented all analyses of the computed phase space trajectory, as described in section 4.1.4. Altogether, our alterations involved writing more than a thousand lines of new code in FORTRAN 90.

Linux boxes, either with two Dual-Core Intel Xeon® 5130 Processors and 2GB RAM on Intel® Dual Socket Server Boards SE7520JR2, or with two Quad-Core Intel Xeon® 5355 Processors and 2GB RAM on Intel ${ }^{\circledR}$ Dual Socket Server Boards S5000PAL, were used to run the code. Since the run-time of the DL_POLY Package scales only moderately with the number of cores on which the computations are spread, we conducted most of our simulations each on a single core. Then, a typical production run of $75 \mathrm{~ns}$ took about 150 to $300 \mathrm{CPU}$ hours, depending on which box was used. They apply for the default systems with 6780 particles. We waited up to more than a thousand CPU hours for comparable production runs of the largest systems with about 55000 particles. The many analyses of the computed trajectories demanded an additional 10 to 50 per cent of the simulation runtime. 


\section{Results}

Various simulations, see Figures 22 and 24, have been conducted in the course of this work. All information on the setup, the preparation, the simulation, the analysis and the expected behaviour of these systems can be looked up in part $\mathbf{4}$. Here, we shall report our results, structured in five main sections. Note that we shall also give, in addition to the observables themselves, their standard deviations as a crude measure for the noise in the data. Its various causes were distinguished previously in part 3 .

Section 5.1 will discuss the values of the global observables and the distributions of the local observables in our first simulations. Above all, we shall demonstrate that the prerequisite for our studies, namely the approximation of reality by our systems, is fulfilled to a large extent. In the nonequilibrium systems, minor anomalies occur due to the thermostating. They fade, however, quickly near the thermostated regions, except for the $z$-dependence of the temperature $y$-gradient in the nonequilibrium interfacial system. We shall find this $z$-dependence to result from a malfunction of our thermostating algorithm. It neglects the spatially variable thermal conductivity perpendicular to the interfaces. Nevertheless, such systems qualify for semiquantitative studies where the error is unimportant or itself subject to research. Thus, we shall discuss in chapter $\mathbf{5 . 2}$ mainly the influences of various technical simplifications on the system behaviour. In particular the differences in the distributions of the local observables often prove to be quite subtle. In order to ease the understanding of these differences, we will preferably compare corresponding equilibrium and nonequilibrium systems together according to a two-part scheme: The equilibrium systems are compared at first. Afterwards, we contrast the deviations of the nonequilibrium systems from their corresponding equilibrium systems. See, for example, section 5.2.1, where several interfacial systems with different cut-off radii are compared in this way.

More advanced quantitative studies would, however, be biased by the malfunction of our thermostating algorithm. Hence, we simulated in the later systems only mixtures with identical particle masses and with identical LJ parameters for the interactions of like particles (see chapter 5.3). We shall call such interfacial systems simply "homophasic" to distinguish them from the "heterophasic" ones studied before. In most simulations, the particle parameters were chosen to be the ones frequently used for $\mathrm{Ar}$ in the literature, e.g. in [michels1949], [hansen1969], or 
[kofke1993]. We thus ensure also better comparability of our results against third party data. In additional simulations we explored different influences on the system behaviour by systematic variation of the system parameters (see chapter 5.4). Some of them could be considered as physical, i.e. they relate directly to the system setup, such as the external constraints for instance.

Chapter 5.5 will then discuss selected aspects in the behaviour of the homophasic nonequilibrium interfacial systems once again in more detail. Thus, we shall compare their density distributions with ones constructed from several related equilibrium interfacial and nonequilibrium one-phase systems. As a result, we can confirm again the validity of our thought experiment from section 4.2.3. The differences correlate with the particle specific streaming velocities in a non-equilibrium interfacial system. Afterwards, we shall examine the local stresses in it. Their different contributions to the local forces add to zero, as required by the Navier-Stokes equation for the small Reynolds numbers in the system.

Finally, we shall consider flows at other types of interfaces that are subject to a lateral temperature gradient (see chapter 5.6). They have many local properties in common if dense fluids are involved. In particular, the flows from hot to cold always seem to occur close to where also the highest density $y$-gradients exist. 


\subsection{Approximation of Reality by our First Systems}

(first heterophasic nonequilibrium interfacial and its corresponding systems)

Here, we shall demonstrate exemplarily the approximation of reality by our simulations by using the results of the first systems. The demonstration proceeds in parallel with chapter $\mathbf{4 . 2}$ which described the general behaviour to expect in each type of system. That is, before we can come to the nonequilibrium interfacial system, which is at the focal point of our studies, the corresponding equilibrium interfacial (see section 5.1.1) and nonequilibrium one-phase systems (see section 5.1.2) are addressed first. Remember from section 4.4.2 that the latter reproduce the phases of the equilibrium interfacial system separately and apply to them the same thermostats and temperature constraints as in the nonequilibrium interfacial system. Based on the existing experience with the general behaviour of the equilibrium interfacial and nonequilibrium one-phase systems, we shall establish the approximation of reality by their simulations. Concurrently, the quantitative simulation results complement the outcome of our thought experiment on the expected behaviour of the nonequilibrium interfacial system (see section 4.2.3), e.g. as to what concerns the peculiarities of the Soret effect in the phases or the magnitude of the variations in the interfacial regions. Finally, we will establish the approximation of reality by this system in comparing its expected behaviour to the observed one (see section 5.1.3).

Note that we will first give the distributions of the local observables, as an exception, without averaging, i.e. as three dimensional plots over $y$ and $z$, so that the reader may assure himself of the quality of the data. Some features in the distributions are, however, too intricate to be easily recognised from the three-dimensional plots. We therefore additionally averaged the local data according to our scheme from section 4.3.5, so as to represent important spatial variations in easy to read two-dimensional plots over the $y$ or $z$. In particular the distributions of the observables that relate to the locally applicable minority component will turn out to be subject to a relatively strong noise. If these distributions allow it qualitatively, we shall therefore assume supplementarily that they have the same features as the corresponding distributions of the majority component. Thus, we shall assume, for instance, if the variation of the minority component densities is linear, that the same holds also for the minority component densities, although the relative magnitude of the fluctuations in them may be much larger. Apart from the local observables also the global ones will be reported, for the sake of completeness, and the standard deviations of all data. 


\subsubsection{Equilibrium Interfacial System}

We shall assess here primarily the approximation of reality by the first interfacial system, EAr1Ar2-0.6-1.0-3346-3524-4.74x8.00x9.40-120-ber. Moreover, it will be exemplified how the simulation results were compiled as a basis for comparison to the corresponding nonequilibrium one-phase and nonequilibrium interfacial systems in the next sections. In the assessment we used two criteria, which determine also the structure of this section. At first, we shall look at the qualitative distributions of the local observables and their standard deviations to see if they accord to our expectations from section 4.2.1. In addition, we will check the quantitative consistency of some results by using different established relations between individual local observables, such as the BGY equation (4-4) or by comparing their spatial averages to the related global observables. This requires, however, reliable data with little noise so that we average and redisplay them before the check. Since both criteria will prove to be fulfilled to a large extent in the subsections below, we shall assume that the equilibrium interfacial system behaves sufficiently realistic for our studies. Finally, the global observables themselves will be reported for the sake of completeness.

\section{Local Observables}

Figure 34 shows the distributions of the local observables without averaging. As established already in section 4.4.1, our thermostating algorithm itself induces no convection. Instead, the local com velocity components (see Figure 34e,f) are homogeneously distributed on a level with the ones of the system which were initially set to zero. Moreover, we stated already in section 4.3.4, when we used slabs to determine the temperature and density distributions, that they accord to our expectations from section 4.2.1. In the figures above these observables show in principle the same behaviour, no matter that they were obtained for cuboid subvolumes now. Thus, the partial densities (see Figure 34b,c) vary sigmoidally in going from one phase to the other, while their sums, i.e. the overall densities, have a vacuous gap (see Figure 34a) and the temperatures remain constant (see Figure 34d). However, one can now see, in addition to the z-dependence, the homogeneity in $y$ direction. This is, of course, at the price of a lower $z$-resolution. It manifests itself clearly in the different shapes of both vacuous gaps. Nevertheless, the cuboid dimensions are still small enough to catch the characteristic $z$-variation of the system, even in the strongly heterogeneous interfacial regions. One can tell this, for instance, from Figure $17 \mathrm{~b}$ where only the density minimum in the 
vacuous gap deviates significantly from the same minimum obtained for a higher z-resolution. Hence, we shall simply continue assessing the approximation of reality based on the cuboid-related data. They also serve as a reference for the other simulations to come.

The distributions of the other observables accord as well to our expectations. Thus, the interatomic $y$-forces, $l_{\mathrm{y}, \mathrm{Ar} 1}$ and $l_{\mathrm{y}, \mathrm{Ar} 2}$, are approximately zero throughout the entire system (see Figure $34 \mathrm{i}, \mathrm{j}$ ). The same holds for the $z$-forces, $l_{z, \operatorname{Ar} 1}$ and $l_{\mathrm{Z}, \mathrm{Ar} 2}$, except in the interfacial regions (see Figure $34 \mathrm{k}, 1$ ). There, $z$-forces of up to approximately $12 \mathrm{pN}$ apply which converge strictly monotonously to zero away from the interfaces and which are always directed into the nearest phase where the particle in consideration constitutes the majority component. The interatomic volume forces, $\mathbf{i}$, vary according to their relation with the partial densities and the particle forces,

$\mathbf{i}=\rho_{A r 1} \mathbf{l}_{A r 1}+\rho_{A r 2} \mathbf{l}_{A r 2}$,

throughout the entire system (see Figure 34g,h). Finally, the normal stresses lie on the order of the negative system pressure. The ones perpendicular to the interfaces, $S_{z z}$, are homogeneously distributed (see Table 6) throughout the entire system, while the lateral normal stresses, $S_{x x}$ and $S_{y y}$, go through a maximum in each interfacial region (see Figure $34 \mathrm{~m}$ ). The fact, that all the distributions above accord to our expectations, supports the assumption that the behaviour of the equilibrium interfacial system approximates reality.

Before we continue with the standard deviations in the next subsection, we first report here explicitly the variations of the different contributions to the normal stresses. We shall take these variations into consideration when checking the consistency of our simulation results further below. The interatomic normal stresses perpendicular to the interface, $I_{z z}$, have a minimum in each interfacial region, while the lateral ones, $I_{x x}$ and $I_{y y}$, have a maximum (see also Figure $34 \mathrm{~m}$ ). They all converge against a phase-dependent common limiting value away from the interfaces. The distributions of the kinetic contributions, $K_{x x}, K_{y y}$, and $K_{z z}$, lie on top of each other and add at any $z$ location with the interatomic normal stresses in the same direction to the normal stresses, $S_{x x}, S_{y y}$, $S_{z z}$. Only the ones lateral to the interfaces exhibit maxima there, while the ones perpendicular to the interfaces remain constant. Hence, they feature only reduced local pressures, which will arise clearly when we compare in section 5.1.2 the global pressures of the present equilibrium interfacial system and its corresponding nonequilibrium one-phase systems. 


\section{Standard Deviations of the Local Observables}

Table 6 contains, as a reference, also the system-averaged standard deviations of all the local observables above. In addition, Figure 34a-h shows the spatial distributions of these standard deviations. In the interfacial regions, all these standard deviations vary in the shape of a "dike". Moreover, they converge against different limiting values away from the interfaces, in analogy with the local observables themselves. Part 8 will address several formulas used to estimate in equilibrium systems the natural fluctuations in the overall densities, temperatures, and com velocities. The standard deviations of these observables vary in our simulation at least qualitatively in keeping with their influence on the estimates, despite the additional noise inherent to the method. Thus, the standard deviations in the Ar1 rich phase slightly exceed those in the Ar2 rich phase where the density is larger. Also, the dikes in the interfacial regions correlate with the vacuous gaps.

In the case of the local com velocities, we can even check the quantitative validity of the estimation formula (8-4). It involves only observables that we determined in the simulation. Let us, for instance, compute the standard deviations in the phases:

$$
\begin{aligned}
& \phi\left[\sigma\left(v_{y}\right): A r 1\right]=\phi\left[\sigma\left(v_{z}\right): A r 1\right]=\sqrt{\frac{1.38 \cdot 10^{-23} \frac{\mathrm{J}}{\mathrm{K}} \cdot 120.2 \mathrm{~K}}{4.74 \mathrm{~nm} \cdot 0.80 \mathrm{~nm} \cdot 0.26 \mathrm{~nm} \cdot 19.13 \mathrm{~nm}^{-3} \cdot 6.634 \cdot 10^{-26} \mathrm{~kg}}}=36.3 \frac{\mathrm{m}}{\mathrm{s}} \\
& \phi\left[\sigma\left(v_{y}\right): \operatorname{Ar} 2\right]=\phi\left[\sigma\left(v_{z}\right): \operatorname{Ar} 2\right]==\sqrt{\frac{1.38 \cdot 10^{-23} \frac{\mathrm{J}}{\mathrm{K}} \cdot 120.0 \mathrm{~K}}{4.74 \mathrm{~nm} \cdot 0.80 \mathrm{~nm} \cdot 0.26 \mathrm{~nm} \cdot 19.95 \mathrm{~nm}^{-3} \cdot 6.634 \cdot 10^{-26} \mathrm{~kg}}}=35.6 \frac{\mathrm{m}}{\mathrm{s}} .
\end{aligned}
$$

We used the temperatures and densities from Table 8 in the computations. They produce values that approximate the standard deviations obtained in the simulation, also given in the table, quite well, i.e. by less than $1 \%$. Note, while the estimates are slightly too small in the Ar1 rich phase, they are slightly too large in the Ar2 rich phase. This might result from long ranged correlations of the com velocity fluctuations in both phases.

The above-mentioned qualitative and, in the case of the com velocities approximately also quantitative, validity of the estimation formulas from part $\mathbf{8}$ supports as well the approximation of reality by our equilibrium interfacial systems. 


\section{Averaging and Further Discussion of the Local Data}

As described in section 4.3.5, we averaged the simulation results to obtain more reliable data. They serve as a reference for comparison to other simulations. Let us have another look at these data before we check their quantitative consistency below. Due to the system's homogeneity in $y$ direction, we can average the local observables at common $z$-coordinates and, in addition, the normal stresses lateral to the interfaces, $S_{x x}$ and $S_{y y}$. Figure 35 shows the resulting data as function of $z$. The partial density z-profiles can be closely approximated by the hyperbolic tangent functions, as we expected in section 4.2.1. We show both the fitted density $z$-profiles and their underlying data in Figure 35a in order to demonstrate the high degree of approximation. Table 10 lists the parameters of these profiles, namely the interfacial width and the $z$-locations of the Gibbs dividing surfaces. No significant variation in the partial density z-profiles can be recognised beyond a distance of two times the interfacial width, $\omega=0.57 \mathrm{~nm}$, from the $z$-locations of the Gibbs dividing surfaces, $\xi_{-}=-2.3 \mathrm{~nm}$ and $\xi_{+}=2.4 \mathrm{~nm}$. This distance thus renders suitable to demarcate the "phases", as defined in section 4.3.5. Accordingly, we shall consider the 160 central subvolumes between the interfaces as phases. Selected local observables are additionally averaged in them. Table 8 lists the values of the thus obtained phase, or in brief P-, averages. They show, as it was found in the work of Buhn et al. [buhn2004a], [buhn2004b], that the stronger interacting Ar2-particles form a phase with a higher overall and majority component density and a lower minority component density. The local standard deviations were averaged in the same way as their related observables and are given as well in the tables above. Figure $35 \mathrm{f}$ shows the $\mathrm{z}$-dependence of the normal stresses perpendicular to the interface, $S_{z z}$, and of averaged ones lateral to the interfacce, $S_{x x y y}$. In the phases, the lateral normal stresses fall approximately $2.4 \mathrm{MPa}$ below the negative global system pressure and exceed it by more than $17 \mathrm{MPa}$ in the interfacial regions. The perpendicular normal stresses lie throughout the entire system on the order of the negatibe global system pressure. Table 10 lists the interfacial tension. In Figure 35c, we show additionally a vector plot of the local com velocities, as obtained by spatially averaging the data at common $z$ - and absolute $y$-coordinates only (see section 4.3.5). The interfacial tension and the vector plot are for comparison to subsequent simulations. 


\section{Consistency of the Simulation Results}

The validity of the physical relations between the observables of a simulation hint at a good approximation of reality. Several relations suggest themselves for such consistency checks in our case. Thus, Figure $35 \mathrm{~d}$ compares the interatomic volume $z$-forces, $i_{z}$, determined directly in the simulation, to the kinetic ones, $k_{z}$, computed from the densities and the temperatures according to equation (4-3). Moreover, Figure 35d also shows the same forces as obtained from the $z$-gradient of the interatomic normal stresses, $\partial I_{z z} / \partial z$, or the negative $z$-gradient of the kinetic normal stresses, $-\partial K_{z z} / \partial z$. Similarly, Figure 35e compares the interatomic particle $z$-forces, $l_{z, A r 1}$ and $l_{z, A r 2}$, obtained from the force conversion subsequent to the simulation (see section 4.3.5) to the ones, $k_{B} T \partial \ln \rho_{A r 1} / \partial z$ and $k_{B} T \partial \ln \rho_{A r 2} / \partial z$, computed from the partial density profiles according to the BGY equation (4-4) from section 4.2.1. In each of the above-mentioned figures, the different results agree quite well. Significant deviations occur only at the centres of the interfacial regions where the spatial resolution of the observables is too low to catch the variation of the system entirely. For the sake of brevity, we do not give any further results here, but these deviations decrease if the observables are determined at an even higher spatial z-resolution, e.g. by using slabs as explained in section 4.3.4. We shall thus generally assume consistent results in the equilibrium interfacial system.

The consistency of our simulation results can also be checked by comparing the spatial averages of the local observables (see Table 6) to their global values (see Table 14). Thus we stated already in section 4.4.1 that the local com velocities or temperatures are homogeneously distributed on a level with the ones of the entire system. Integration of the local pressures, $\Pi$, across the entire system and averaging the resulting integrals according to the relation,

$\varnothing[\Pi: S]=\frac{\int_{-0.5 L_{z}}^{0.5 L_{z}}(\Pi) \mathrm{d} z}{L_{z}}=-\frac{\int_{-0.5 L_{z}}^{0.5 L_{z}}\left(\frac{S_{x x}+S_{y y}+S_{z z}}{3}\right) \mathrm{d} z}{L_{z}}$,

produces the global system pressure. It must formally equal the one, $P$, computed via the virial route (see section 4.3.1). All the global observables above deviate from their related spatial system averages by less than $0.2 \%$ or $0.002 \mathrm{~m} / \mathrm{s}$ in the case of the com velocities. Such deviations lie within the uncertainty due to round-off errors in the computation of the values. In addition, more phase points, i.e. every hundredth, are considered in the computation of the local temperatures or normal 
stresses. We shall therefore assume that the values of the local and of the global observables are consistent.

Let us mention, for the sake of completeness, two more conclusions from the established consistency. The one between the different local observables demonstrates the validity of our way to compute the interatomic forces per particle. Remember from section 4.3.3 how we determine them only after a simulation by dividing the mean force on all particles of a certain substance through their quantity in a subvolume. We must however keep in mind the relatively strong noise in the forces per particle of the minority component. Thus, their standard deviations, $\varnothing\left[\sigma_{s}\left(l_{A r l}\right): A r 2\right]$ and $\varnothing\left[\sigma_{s}\left(l_{A r 2}\right): A r l\right]$, exceed, for instance in the phases, more than ten-times the one of the forces per majority particle, $\varnothing\left[\sigma_{s}\left(l_{A r 1}\right): A r 1\right]$ and $\varnothing\left[\sigma_{s}\left(l_{A r 2}\right): A r 2\right]$ (see Table 8). The second conclusion relates to the interfacial density profiles. Figure 35e shows additionally the mean particle forces obtained by plugging the fitted hyperbolic tangent partial density profiles into the BGY equation, $l_{z, A r l, \rho-f i t}$ and $l_{z, A r 2, \rho-f i t}$. These variations have about $25 \%$ smaller absolute extreme values in the interfacial regions, which suggests that the fitted hyperbolic tangent density profiles are somewhat too flat.

\section{Global Observables and their Standard Deviations}

Let us take, for the sake of completeness, a brief look at the values of the global observables themselves and their standard deviations (see Table 14). The pressure has, relatively speaking, the strongest fluctuations in comparison to the system energy or temperature, which is typical for $N V E$ or NVT simulations of liquids. The heat flux into or out of the cold thermostated region approximates the expectation value, zero, by less than one standard deviation. Note that the temperature of the region, which comprises the entire system in the NVT simulation as stated in section 4.1, is slightly lower than the system temperature due to the different number of degrees of freedom considered (compare sections 4.3.1 and 4.1.4). Apart from this difference the system temperate constraint is closely met with a standard deviation of $\sigma(T) 0.003 \mathrm{~K}$. 


\subsubsection{Nonequilibrium One-Phase Systems}

We shall assess here to what extent the simulations of the first nonequilibrium one-phase systems, N-Ar1Ar2-0.6-1.0-6795-24-4.74x8.00x9.40-100-140

and N-Ar1Ar2-0.6-1.0-20-7091-4.74x8.00x9.40-100-140,

approximate reality. This approximation can, due to the unphysical nature of our thermostats, apply only remote from them in the actual investigation regions. We will use in the assessment the same two criteria as in the equilibrium interfacial system from the previous section: do the systems behave as could be expected from previous studies (see section 4.2.2) and are the local simulation results consistent? First, we shall compare the expected behaviour with the distributions of the local observables. Afterwards, we shall check their consistency by using the averaged data. Both criteria will turn out to be sufficiently fulfilled for our further studies. Finally, we will report the global observables for the sake of completeness.

\section{Local Observables}

Figure 34 shows the distributions of the local observables without averaging in three-dimensional plots. We established already in section 4.4.2 that our thermostating algorithm itself induces no convection: The local com velocity components (see Figure 34e,f) are, as expected, homogeneously distributed on a level with the ones, set to zero, of the entire systems. Moreover, we stated already in section 4.3.4, when we used slabs to determine the temperature and density distributions, that they agree in the investigation regions with our expectations from section 4.2.2. In the figures mentioned above these observables show the same behaviour, except that we see additionally their homogeneity in $z$-direction, now. Thus, the densities and temperatures are constant in $z$-direction and vary linearly in $y$-direction between the thermostated regions (see Figure 34a,b,c,d): The overall and majority component densities decrease in going from the cold to the hot region, while the minority component densities and the temperatures increase. Since the $y$-variations are linear, they are expressed as well, i.e. without loss of detail, for the cuboid subvolumes despite the lower resolution. Hence, we shall simply continue the analysis based on the cuboid data, as in the equilibrium interfacial system from the previous section. The cuboid data also have the additional advantage that the unphysical behaviour near and inside the thermostated regions is smeared out to some extent, as it will be discussed further below. 
Also the distributions of the other observables agree with our expectations from section 4.2.2. Thus, when disregarding the relatively strong noise in the interatomic volume forces, their $z$-components are approximately zero throughout each system while the $y$-components always point to the nearest hot thermostated region (see Figure 34g,h). The same holds for the interatomic particle forces (see Figure 34i-1), the $y$-forces per minority particle clearly exceeding the ones per majority particle, as expected. Figure 35e and Table 8 will show this below more clearly for the averaged data. Finally, the different normal stresses in the investigation regions of each system (see Figure $34 \mathrm{~m}$ ) are homogeneously distributed on the order of the negative global pressure (see Table 14), as we shall see below. Both the interatomic and kinetic contributions to the normal stresses vary linearly with identical absolute slopes between the thermostated regions, the kinetic contribution decreasing in going from the cold to the hot thermostated region. The fact, that all distributions above agree qualitatively, and as to the orders of magnitude also quantitatively, with our expectations, supports the assumption that the qualitative behaviour of the actual investigation regions in the nonequilibrium one-phase systems approximates reality.

Before we move on to the standard deviations, we would like to mention, for the sake of completeness, that there are minor fluctuations in the normal stresses, particularly in the ones perpendicular to the temperature gradient, $S_{x x}$ and $S_{z z}$, near the thermostated regions (see Figure $34 \mathrm{~m})$. These fluctuations correlate with the particle layering already described (see section 4.3.4). They are, however, small inside the investigation region and can therefore be neglected in our further considerations.

\section{Standard Deviations of the Local Observables}

Tables 6-13 list also the standard deviations of the local observables above for further reference. The values resemble remarkably those in the equilibrium interfacial system from the previous section, which suggests that the additional numerical and simulation errors in the NEMD simulations have only little impact on the reliability of the local data.

In addition, Figure 34a-h shows the standard deviations of the instantaneous function values, that underlie the local observables, as function of $y$ and $z$. All these distributions increase with $y$ in 
approaching the hot region. The standard deviations of the overall densities, temperatures, and com velocities vary at least qualitatively in keeping with the estimation formulas for equilibrium systems (see part 8). Thus, the relative increase of the density standard deviations in $y$-direction, for instance, is only marginal. This is in keeping with the contrary influences of the temperature and density variation in the estimation formula (8-3). On the other hand, both variations act strongly promotive in the estimation formula of the temperature standard deviations, see equation (8-1), which show accordingly the strongest relative increase.

Equation (8-4) allows us to estimate the com velocity standard deviations of in the corresponding equilibrium interfacial system. They must be less than $1 \%$, based its the local temperatures and densities. We shall use here the same expression to estimate the extreme values of the standard deviations in the nonequilibrium one-phase systems:

N-Ar1Ar2-0.6-1.0-6795-24-4.74x8.00x9.40-100-140

$$
\begin{aligned}
& \phi\left[\sigma\left(v_{y}\right):|y|=3.6 \mathrm{~nm}\right]=\phi\left[\sigma\left(v_{z}\right):|y|=3.6 \mathrm{~nm}\right] \approx \\
& \approx \sqrt{\frac{1.38 \cdot 10^{-23} \frac{\mathrm{J}}{\mathrm{K}} \cdot 134.64 \mathrm{~K}}{4.74 \mathrm{~nm} \cdot 0.80 \mathrm{~nm} \cdot 0.26 \mathrm{~nm} \cdot 18.29 \mathrm{~nm}^{-3} \cdot 6.634 \cdot 10^{-26} \mathrm{~kg}}}=39.4 \frac{\mathrm{m}}{\mathrm{s}} \\
& \phi\left[\sigma\left(v_{y}\right):|y|=0.4 \mathrm{~nm}\right]=\phi\left[\sigma\left(v_{z}\right):|y|=0.4 \mathrm{~nm}\right] \approx \\
& \approx \sqrt{\frac{1.38 \cdot 10^{-23} \frac{\mathrm{J}}{\mathrm{K}} \cdot 105.03 \mathrm{~K}}{4.74 \mathrm{~nm} \cdot 0.80 \mathrm{~nm} \cdot 0.26 \mathrm{~nm} \cdot 19.93 \mathrm{~nm}^{-3} \cdot 6.634 \cdot 10^{-26} \mathrm{~kg}}}=33.3 \frac{\mathrm{m}}{\mathrm{s}}
\end{aligned}
$$

N-Ar1Ar2-0.6-1.0-20-7091-4.74x8.00x9.40-100-140

$$
\begin{aligned}
& \phi\left[\sigma\left(v_{y}\right):|y|=3.6 \mathrm{~nm}\right]=\phi\left[\sigma\left(v_{z}\right):|y|=3.6 \mathrm{~nm}\right] \approx \\
& \approx \sqrt{\frac{1.38 \cdot 10^{-23} \frac{\mathrm{J}}{\mathrm{K}} \cdot 134.82 \mathrm{~K}}{4.74 \mathrm{~nm} \cdot 0.80 \mathrm{~nm} \cdot 0.26 \mathrm{~nm} \cdot 19.23 \mathrm{~nm}^{-3} \cdot 6.634 \cdot 10^{-26} \mathrm{~kg}}}=38.5 \frac{\mathrm{m}}{\mathrm{s}} \\
& \phi\left[\sigma\left(v_{y}\right):|y|=0.4 \mathrm{~nm}\right]=\phi\left[\sigma\left(v_{z}\right):|y|=0.4 \mathrm{~nm}\right] \approx \\
& \approx \sqrt{\frac{1.38 \cdot 10^{-23} \frac{\mathrm{J}}{\mathrm{K}} \cdot 105.24}{4.74 \mathrm{~nm} \cdot 0.80 \mathrm{~nm} \cdot 0.26 \mathrm{~nm} \cdot 20.64 \mathrm{~nm}^{-3} \cdot 6.634 \cdot 10^{-26} \mathrm{~kg}}}=32.8 \frac{\mathrm{m}}{\mathrm{s}} .
\end{aligned}
$$


Since the differences between the resulting estimates, which are based on the $\mathrm{C}$ - and $\mathrm{H}$-averages of the temperatures and densities in Table 9, and the standard deviations obtained in the simulation, also given in the table, amount to less than $1 \%$, we assume that the estimation formulas are valid. This makes, together with the above-mentioned qualitative validity of the other estimation formulas, the distributions of the com velocity standard deviations and thus the behaviour of both systems appear plausible from a physical point of view.

\section{Averaging and Further Discussion of the Local Observables}

As explained in section 4.3.5, the nonequilibrium one-phase systems exhibit a homogeneity in $z$ direction and a symmetry with respect to the $x z$-plane. We took advantage of both by averaging the local observables at common absolute $y$-coordinates and, in addition, the normal stresses perpendicular to the temperature gradient, $S_{x x}$ and $S_{z z}$. Moreover, I-averages of the interatomic volume and particle forces were computed to obtain more reliable values at $|y|=2.0 \mathrm{~nm}$ (see Table 7). We shall check further below the consistency of our simulation results by using these averaged data. Figure 35 displays them as function of $y$. We also computed the $y$-gradients of selected observables (see Tables 12-13). In addition, Table 9 lists the explicit $\mathrm{C}-, \mathrm{M}-$, and $\mathrm{H}$-averages at certain $y$ coordinates. We can tell from the averaged density $y$-profiles (see Figure 35a) more clearly how the thermal diffusion works contrariwise in both systems. The particles of the majority component always concentrate at the cold thermostated region while the ones of the minority component concentrate at the hot thermostated region, see Table 11 for the related Soret coefficients. This separation correlates with the proportions of the interatomic particle $y$-forces, the ones per minority particle clearly exceeding the ones per majority particle.

The unphysical system behaviour near the thermostated regions disappears to some extent in the distributions of the local observables, as obtained for the cuboid subvolumes. We can tell so by comparing these distributions to the ones obtained in section 4.3.4 where slabs were used. For instance, the strong temperature variations and the layering of the particles near the thermostated regions cannot be recognised anymore. Instead, the temperatures and densities determined for the cuboids, that contain parts of the thermostated regions, now strongly resemble the data, that is obtained by extrapolating the density and temperature $y$-profiles from the investigation regions (see Figure $35 \mathrm{a}, \mathrm{b})$. In our subsequent studies we shall therefore consider, additionally, the temperatures and densities for the cuboids outside the investigation regions, if necessary to make a point. The 
local volume or particle $y$-forces there, on the other hand, continue to be too strongly biased to be taken as well into consideration. Their values incorporate the high unphysical force peaks seen near the thermostated regions (see section 4.3.4) and hence are clearly larger than in the actual investigation region (see Figure 35d,e). Inside the systems however, the different spatial resolution hardly manifests itself in any of the local observables. Thus, the slopes of the temperature and of the overall and majority component density $y$-profiles differ by less than $1 \%$ from the slopes computed from the slab-related data. Concurrently, the values of these profiles at $|y|=2.0 \mathrm{~nm}$ are even closer to each other. Figure $35 \mathrm{f}$ shows the normal stresses lateral to the temperature gradient, $S_{y y}$, together with the averaged perpendicular ones, $S_{x x z z}$. In Figure 35c, we show additionally a vector plot of the local com velocities, as obtained by spatially averaging the data at common $z$ - and absolute $y$ coordinates only (see section 4.3.5). This vector plot is for comparison with the subsequent simulations.

\section{Consistency of the Simulation Results}

We shall first check the consistency of the simulation results by comparing the kinetic and interatomic $y$-forces in the system. Both must add to zero everywhere (see chapter $\mathbf{2 . 2}$ and section 4.2.2). Since the different $y$-forces are subject to a relatively strong noise, we shall compare only their I-averages. Table 7 lists them for the interatomic volume $y$-forces, $i_{y}$, determined directly from the simulation, and the kinetic ones, $k_{y}(\rho, T)$, computed from the density and temperature distributions (equation (4-14)). In addition, the same forces are given as obtained by spatially averaging the $y$-gradients of the interatomic normal stresses, $\partial I_{y y} / \partial y$, or the negative $y$-gradients of the kinetic normal stresses, $-\partial K_{y y} / \partial y$, over the investigation regions (equation (2-2)). We shall assume, based on the quite good agreement of the different results, that our local data are consistent. It exists similarly also in the case of the particle forces, i.e. the interatomic particle $y$-forces, $l_{y, A r l}$ and $l_{y, A r 2}$, determined from the simulation to the ones obtained from equations (4-16), $l_{y, A r l}\left(\rho_{A r l}, T\right)$, and (4-17), $l_{y, A r 2}\left(\rho_{A r 2}, T\right)$.

We can additionally check the consistency of our simulation results by comparing the system averages of selected local observables (see Table 6) to their global values in Table 14. As stated previously in section 4.4.2, the local com velocities, for instance, are homogeneously distributed on a level with the ones of an entire system. Moreover, the global system pressures, obtained by averaging the negative local normal stresses according to equation (5-1), deviate by less than $0.5 \%$ 
from the ones determined directly in the simulations. Apparently, the spatial fluctuations in the normal stresses near both thermostated regions cancel each other to a large extent.

\section{Global Observables and their Standard Deviations}

Different from the previous section, where only one of the thermostats (the cold one) provided by our algorithm was concerned with an entire system, now both thermostats maintain small regions of a system at different temperatures. Accordingly, there should be a macroscopically constant flux of heat between both thermostated regions. It is continuously added in the form of kinetic energy to the hot region and removed at the same rate from the cold one. Our nonequilibrium one-phase systems show exactly this behaviour, except that the absolute values of the two fluxes determined in the same simulation differ somewhat (see Table 14). When extrapolated to the length of the simulations $L_{t}=75 \mathrm{~ns}$, this difference corresponds to a huge amount of energy, compared to the one of the entire system, for instance

$$
\left(\left|\mu\left(Q_{H}\right)\right|-\left|\mu\left(Q_{C}\right)\right|\right) L_{t}=(108.3 n W-101.2 n W) \cdot 75 n s=-532.5 a J
$$

compared to $\mu(E)=-64.69 \mathrm{aJ}$ in N-Ar1Ar2-0.6-1.0-6795-24-4.74x8.00x9.40-100-140. Such an extrapolation has, however, little significance since the heat fluxes exhibit also relatively strong fluctuations in the table. Their magnitudes imply that the thermostats overmodulate in that the amounts, by which the kinetic energies of the concerned thermostated regions are altered each time step to maintain the intended temperature, clearly exceed the actual heat flux through the systems. Thus, we show exemplarily in Figure 36 an excerpt of the instantaneous heat fluxes of the cold thermostated region in N-Ar1Ar2-0.6-1.0-6795-24-4.74x8.00x9.40-100-140. They frequently even become positive. The fluctuations are even larger at the hot thermostated region. This overmodulation biases the other system behaviour, however, only little so that it can establish realistically in the intermediate regions as shown above. Moreover, we demonstrated already in section 4.4.2 that the behaviour is also stationary with respect to the global observables and the distributions of the local observables. Nevertheless, the values of the transport rates must be regarded with suspicion due to their large fluctuations. Note that they are much smaller in the equilibrium interfacial system from the previous section, where our thermostats were applied to implement a Berendsen thermostat. Likewise, we shall obtain more reliable results for the heat fluxes in NEMD simulations by using the loose version of our thermostats (see section 5.2.3). 
The additional numerical and simulation errors, that accompany an NEMD simulation, manifest themselves also in the standard deviations of the other global observables (see Table 14). Their magnitudes exceed, in the case of the global temperatures, the ones in the equilibrium interfacial system from the previous section by more than $65 \%$. However, these errors compensate themselves to a large extent for the systems as a whole, so that the global pressures and temperatures resemble those in the corresponding equilibrium interfacial system. A slight drop can be noticed in the global temperatures. Such drops result from the previously described (see section 4.3.4) different magnitudes and extensions of the temperature jumps at the thermostated regions. On the other hand, the global pressures of the nonequilibrium one-phase systems slightly exceed the one of their corresponding equilibrium interfacial system due to its reduced interfacial pressures (see section 5.1.1). If they are disregarded by spatially averaging the local pressures within each phase only, we obtain averages, $\varnothing[\Pi: A r 1]$ and $\varnothing[\Pi: A r 2]$, that approximate the global pressures in the corresponding values nonequilibrium one-phase systems even better. The global energies of all the abovementioned systems vary clearly with their number of Ar2 particles. Their interactions with each other and with other particles feature larger LJ parameters, $\varepsilon_{\mathrm{Ar} 1-\mathrm{Ar} 2}$ and $\varepsilon_{\mathrm{Ar} 2-\mathrm{Ar} 2}$, and thus entail, on balance, smaller system energies the more Ar2 particle are abundant. 


\subsubsection{Nonequilibrium Interfacial System}

We shall assess here, in analogy with the equilibrium interfacial and nonequilibrium one-phase systems from the previous two sections, the approximation of reality by our first nonequilibrium interfacial system, N-Ar1Ar2-0.6-1.0-3346-3524-4.74x8.00x9.40-100-140-r1. Thus, we shall first check the qualitative system behaviour against the expected one from our thought experiment in section 4.2.3. Both will turn out to agree quite well except for a minor temperature anomaly. Afterwards, we shall look at the summarised local data. Since they are consistent, this anomaly must result from a malfunction of our thermostats that did not occur in the previous equilibrium interfacial and nonequilibrium one-phase systems. We will ascribe it to the different heat conductivities of both phases. Its error on the system behaviour is, however, difficult to disentangle. We shall then establish the different behaviour of the nonequilibrium interfacial system in comparison to the previous equilibrium interfacial and nonequilibrium one-phase ones. Finally, the global observables will be reported for the sake of completeness. Note, we shall use the summarised simulation results as a reference for comparisons with other systems.

\section{Local Observables}

Figure 34 shows the distributions of the local observables as function of $y$ and $z$, without averaging. Let us have a look at the local com velocity components first (see Figure 34e,f). While evenly distributed in the corresponding systems from the previous two sections (scattering around their expectation value zero), systematic trends are now observed. Thus, we can tell unambiguously from the plot of the $y$-velocities that matter flows close to the interfaces from the hot towards the cold region, with compensating counter flows taking place in the centre of each phase. In agreement with the $y$-velocities, the $z$-velocities indicate a convection away from the interfaces in the neighbourhood of the cold thermostated region. Close to the hot thermostated regions, the fluid is drawn back towards the interfaces. These features appear eight times in a simulation cell due to the symmetry required by the periodic system boundaries and demonstrate exactly the expected thermocapillary convection roll cells, as described in section 4.2.3. We shall also see them in the behaviour of all later discussed nonequilibrium interfacial systems (see sections 5.2, 5.4, and 5.5). The magnitudes and orientations of the com velocities will always clearly exceed the fluctuations, $\phi\left[\sigma_{s}\left(v_{y}\right)\right]$ and $\phi\left[\sigma_{s}\left(v_{z}\right)\right]$, in the nonequilibrium interfacial system itself or its corresponding 
equilibrium interfacial and nonequilibrium one-phase systems (see Table 7). We shall thus conclude that the observed convection is a reliable and reproducible one, reflecting a true physical phenomenon. Chapter 5.4 is devoted to the quantification of this observation, which is one of the main findings of this work.

A spatially variable stress distribution comes with the thermocapillary flows. We can tell it particularly well in the case of the perpendicular normal stresses, $S_{z z}$. They increase in going from the cold to the hot thermostated region (see Figure 34m). Since they, in addition to that, should be independent of $z$, we can expect the same gradient to approximate the negative pressure $y$-gradient, $\partial \Pi / \partial y$, in the phases. We shall consider it as an additional measure for the strength of the thermocapillary effect. Section 5.5.2 will address the other aspects of the stress distribution in more detail.

Having demonstrated the occurrence of the thermocapillary convection, we study its impact on the distributions of the other local observables. The maximum com velocities above amount to less than $2 \mathrm{~m} / \mathrm{s}$, so that the system has only a small Péclet number. It was generally explained in section 4.2.3. Correspondingly, these distributions should approximate the ones described in our thought experiment for negligible convective transport; i.e. the field observables such as the temperatures for instance, are constant in $z$-direction while the quasi-intensive observables, such as the densities for instance, converge against constant limiting values away from the interfaces but strongly vary at them. Concurrently, all observables vary approximately linearly in going from the cold to the hot thermostated region so that the $z$-variation of the quasi-intensive observables becomes weaker and wider. As a matter of fact, our results mostly exhibit this behaviour. Thus, as in the corresponding nonequilibrium one-phase systems, the overall and majority component densities decrease linearly in approaching the hot thermostated region while the minority component densities and temperatures increase (see Figure 34a,b,c,d). Moreover, when disregarding the noise in the interatomic $y$-forces per unit volume or per particle, they point toward the nearest hot thermostated region, the ones per minority particle clearly exceeding the ones per majority particle (see Figure $34 \mathrm{~g}, \mathrm{i}, \mathrm{j})$. The densities and the interatomic volume and particle $z$-forces vary in $z$-direction as in the corresponding equilibrium interfacial system (see Figure 34h,k,l). One can tell from the strengths of the variations and from the contour-lines at the bottom of the plots that the interfaces become wider in going from the cold to the hot thermostated region. Only the slight $z$-dependence of the temperatures violates our expectations. We shall see it more clearly in the summarised data further below. Nevertheless, we can already establish here that this temperature anomaly is too small to 
bias significantly the qualitative distributions of the other observables above. Hence, the qualitative system behaviour can be assumed to be realistic, at least as far as they are concerned.

Remember from the nonequilibrium one-phase systems in the previous section that the unphysical system behaviour due to the thermostating manifests itself only partly in the results for the cuboids extending into thermostated regions. The densities and the temperatures, which proved to be also only slightly biased outside the investigation regions, were therefore additionally considered, if necessary to make a point. We shall do the same for the com velocities as well as the interatomic volume and particle $z$-forces in a nonequilibrium interfacial system. Their results resemble the ones obtained by extrapolating the data in the investigation regions.

\section{Local Standard Deviations}

Tables 6-13 list also the standard deviations of all the observables above. Similar to the nonequilibrium one-phase systems from the previous section, the values resemble those in the corresponding equilibrium interfacial system (see section 4.4.2) remarkably, so that the additional errors due to the thermostating have only little impact on the reliability of the local data. Moreover, Figure 34a-h shows the local standard deviations as function of $y$ and $z$. Their distributions are, as in the case of the local observables themselves (see section 4.4.3), a combination of the data in two nonequilibrium one-phase systems joined by the interfacial variations of different equilibrium interfacial systems. Thus, the standard deviations converge against constant limiting values away from the interfaces but vary in the shape of a "dike" at them. Concurrently, the distributions represent the heterogeneity of the system due to its removal from equilibrium in that they increase with $y$ in approaching the hot thermostated region. The temperature anomaly described above is too small to appear qualitatively in the standard deviations. When disregarding it, we can easily establish, similar to the corresponding equilibrium interfacial and nonequilibrium one-phase systems, that the standard deviations of the overall densities, the temperatures, and the com velocities vary at least qualitatively according to their influence in the estimation formulas from part 8. Moreover, the estimates of the com velocity standard deviations differ from the ones determined in the simulation by less than $1 \%$. We give here, as an example, only the extreme estimates in each phase, which were computed from the $\mathrm{C}$ - and $\mathrm{H}$-averages of the temperatures and densities (see Table 9):

$\phi\left[\sigma\left(v_{y}\right): A r 1,|y|=3.6 n m\right]=\phi\left[\sigma\left(v_{z}\right): A r 1,|y|=3.6 n m\right] \approx$ 


$$
\begin{aligned}
& \approx \sqrt{\frac{1.38 \cdot 10^{-23} \frac{\mathrm{J}}{\mathrm{K}} \cdot 135.20 \mathrm{~K}}{4.74 \mathrm{~nm} \cdot 0.80 \mathrm{~nm} \cdot 0.26 \mathrm{~nm} \cdot 18.24 \mathrm{~nm}^{-3} \cdot 6.634 \cdot 10^{-26} \mathrm{~kg}}}=39.5 \frac{\mathrm{m}}{\mathrm{s}} \\
& \phi\left[\sigma\left(v_{y}\right): A r 1,|y|=0.4 n m\right]=\phi\left[\sigma\left(v_{z}\right): A r 1,|y|=0.4 n m\right] \approx \\
& \approx \sqrt{\frac{1.38 \cdot 10^{-23} \frac{\mathrm{J}}{\mathrm{K}} \cdot 104.67 \mathrm{~K}}{4.74 \mathrm{~nm} \cdot 0.80 \mathrm{~nm} \cdot 0.26 \mathrm{~nm} \cdot 19.96 \mathrm{~nm}^{-3} \cdot 6.634 \cdot 10^{-26} \mathrm{~kg}}}=33.2 \frac{\mathrm{m}}{\mathrm{s}} \\
& \phi\left[\sigma\left(v_{y}\right): A r 2,|y|=3.6 n m\right]=\phi\left[\sigma\left(v_{z}\right): A r 2,|y|=3.6 n m\right] \approx \\
& \approx \sqrt{\frac{1.38 \cdot 10^{-23} \frac{\mathrm{J}}{\mathrm{K}} \cdot 133.88 \mathrm{~K}}{4.74 \mathrm{~nm} \cdot 0.80 \mathrm{~nm} \cdot 0.26 \mathrm{~nm} \cdot 19.24 \mathrm{~nm}^{-3} \cdot 6.634 \cdot 10^{-26} \mathrm{~kg}}}=38.2 \frac{\mathrm{m}}{\mathrm{s}} \\
& \phi\left[\sigma\left(v_{y}\right): A r 2,|y|=0.4 n m\right]=\phi\left[\sigma\left(v_{z}\right): A r 2,|y|=0.4 n m\right] \approx \\
& \approx \sqrt{\frac{1.38 \cdot 10^{-23} \frac{\mathrm{J}}{\mathrm{K}} \cdot 105.64 \mathrm{~K}}{4.74 \mathrm{~nm} \cdot 0.80 \mathrm{~nm} \cdot 0.26 \mathrm{~nm} \cdot 20.62 \mathrm{~nm}^{-3} \cdot 6.634 \cdot 10^{-26} \mathrm{~kg}}}=32.8 \frac{\mathrm{m}}{\mathrm{s}} \text {. }
\end{aligned}
$$

Hence, the distributions of the standard deviations confirm, in addition to the ones of the observables themselves, that at least the qualitative system behaviour approximates reality, except for the minor temperature anomaly.

\section{Averaging and Further Discussion of the Local Observables}

We took advantage of the system's symmetry with respect to the $x z$-plane and averaged the local observables at common $z$ - and absolute $y$-coordinates as described in section 4.3.5. The resulting data, which also serve as a reference, are presented here in different two-dimensional graphs (see Figure 35). Their more exact readability makes additional more subtle features in the system behaviour identifiable. At first, we look at the graphs over $\mathrm{z}$.

Figure $35 \mathrm{c}$ shows the averaged local $y$ - and $z$ - com velocity components. The relatively largest absolute $y$-velocities of about $1.8 \mathrm{~m} / \mathrm{s}$ occur in the interfacial regions at $|y|=2.0$ and $|y|=2.8 \mathrm{~nm}$. On the other hand, the relatively largest absolute $z$-velocities of about $0.9 \mathrm{~m} / \mathrm{s}$ occur at $|y|=3.6 \mathrm{~nm}$, about $0.8 \mathrm{~nm}$ away from the interfaces, which is less than $L_{z} / 8$. Accordingly, the centre of each roll cell is slightly shifted towards the nearest interface and the adjacent hot thermostated region. We show this situation once again more clearly in a vector plot (see also Figure 35c). Note that the exact maximum absolute $y$ - and $z$-velocities are additionally listed in Table 11, as a reference. We shall use them as a crude measure for the intensity of the thermocapillary convection (see section 4.2.3). 
Figure 35a displays the density z-profiles at different absolute $y$-coordinates. The interfaces widen, as expected, in going from the cold to the hot thermostated region while their $z$-locations hardly shift in $z$-direction. Moreover, each profile is closely approximated by the same hyperbolic tangent functions as in an equilibrium interfacial system. This holds even outside the investigation regions at the $y$-coordinates of $|y|=0.4 \mathrm{~nm}$ or $|y|=3.6 \mathrm{~nm}$ (see Figure $35 \mathrm{a}$ ). Table 11 gives the thus obtained widths and $z$-locations of the interfaces. Their widths remain far smaller than two times the ones, $\omega(2.0 \mathrm{~nm})=0.56 \mathrm{~nm}$, at the centres between the thermostated regions. Concurrently, the $z$-locations of the interfaces shift only marginally into the Ar2 rich phase, from $\xi_{-}(|y|=0.4 \mathrm{~nm})=-2.27 \mathrm{~nm}$ and $\xi_{+}(|y|=0.4 \mathrm{~nm})=2.39 \mathrm{~nm}$ to $\xi_{-}(|y|=3.6 \mathrm{~nm})=-2.29 \mathrm{~nm}$ and $\xi_{+}(|y|=3.6 \mathrm{~nm})=2.41 \mathrm{~nm}$. We could therefore demarcate the phases in the intended simplistic way, i.e. by treating the nonequilibrium interfacial system as an equilibrium one by using the widths and $z$-locations of the interfaces at the centres between the thermostated regions (see section 4.3.5). Accordingly, the central 160 subvolumes between the phases were lumped together into two phases for additional averaging of selected local observables. We averaged them in each phase, for instance, at all $z$ - and common absolute $y$ coordinates. Table 9 gives the $\mathrm{C}-, \mathrm{M}-$, and $\mathrm{H}$ - averages at certain $y$-coordinates. Moreover, we redisplay the averaged densities at different $y$-coordinates in the phases in Figure 35a. Such a presentation indicates more clearly how both substances separate between the thermostated regions. Our studies concentrate, however, particularly on the interfacial regions. Their density $y$-profiles are hard to include into a single plot without making it illegible. Instead, we prefer another manner of presentation and plot the density $y$-gradients as function of $z$ (see as well Figure 35a). They characterise together with one of the above density $z$-profiles the entire density distribution. We shall use the $z$-profile at $|y|=2.0 \mathrm{~nm}$ for its similarity to the one of the corresponding equilibrium interfacial system. As to the variation of the density $y$-gradients in the interfacial regions, it is "wshaped" in the case of the overall densities or "z-shaped" in the case of the partial densities. The zprofile converges against different constant values away from the interfaces, where the system tends to become homogeneous in $z$-direction. The above characterisation of the density distributions will also prove advantageous for the ones of the temperatures as well as the standard deviations of all the local observables determined in the nonequilibrium interfacial system. In Table 13 we give additionally the phase-averaged $y$-gradients of the densities, the temperatures, and the different standard deviations. The Soret coefficients, computed from these $y$-gradients and from the Maverages above according to section $\mathbf{4 . 3 . 5}$, are given in Table 11 . 
The temperatures in Figure 35b exhibit, at first sight, similar anomalies as in the corresponding nonequilibrium one-phase systems. Thus, the temperatures at $|y|=2.0 \mathrm{~nm}$ are about $1 \mathrm{~K}$ lower than the $120 \mathrm{~K}$ to expect from Fourier's law. Likewise, the temperature $y$-gradients are flatter than expected due to the temperature jumps at the thermostated regions. However, an additional anomaly can be distinguished in the nonequilibrium interfacial system: The temperatures vary sigmoidally between the slightly different limiting values in the phases. The closer a $z$-profile lies to one of the thermostated regions, the stronger deviate its limiting values and the more widens the variation between them. Accordingly, the temperature $y$-profiles clearly differ in both phases (see also Figure $35 \mathrm{~b}$ ). In order to further characterise the temperature distribution in the interfacial regions, we computed, as in the cases of the densities and standard deviations above, its $y$-gradients. They vary sigmoidally in the interfacial regions (see also Figure 35b). Table 13 lists additionally the phase averaged temperature $y$-gradients.

Figure 35d,e redisplays the different $z$-profiles of the interatomic volume and particle force components after the averaging over common $z$ - and absolute $y$-coordinates. The resulting data are nevertheless subject to a relatively strong noise. Hence, we refrained from computing $y$-gradients of the different force-components to describe their variation in the interfacial. Instead, the I-averages of the different force-components were additionally computed at every $z$-coordinate (see also Figure $35 \mathrm{~d}$,e). These averages apply approximately at $|y|=2.0 \mathrm{~nm}$. In order to establish particularly the $y$ dependence of the different interatomic $z$-forces, we compare their values at $|y|=0.4 \mathrm{~nm}$ and $|y|=3.6 \mathrm{~nm}$ to their I-averages. The $y$-dependence of the different interatomic $y$-forces cannot be established at all due to the noise. However, we give additionally in Table 8 their I-averages over each phase. They tell us at least that the $y$-force per minority particle clearly exceeds the one per majority particle, as in the corresponding nonequilibrium one-phase systems.

\section{Consistency of the Simulation Results}

We established previously the small influence of the convective transport in our nonequilibrium interfacial system. Hence, its local forces must be approximately zero in equation (2-1). Moreover, we can estimate them by using the same relations as in the corresponding equilibrium interfacial (see section 5.1.1) and nonequilibrium one-phase systems (see section 5.1.2). In fact, the interatomic volume and particle forces, obtained directly from the simulation, and their associated kinetic forces, that are computed from the density and temperature distributions, add, as expected, 
approximately to zero everywhere (equations (4-14), (4-16)-(4-17)). Thus, we compare in Figure $37 \mathrm{a}, \mathrm{b}$ at the different $z$-coordinates the I-averages of the different $y$-forces. Table 8 lists additionally their I-averages over each phase. Similarly, the different interatomic and kinetic $z$-forces cancel each other except at the centre of each interfacial region. Their spatial resolution is too low to catch the variation of the system there. Figure 37c compares, as an example, the absolute values of these $z$-forces at the absolute $y$-coordinates of $|y|=0.4 \mathrm{~nm}$ and of $|y|=3.6 \mathrm{~nm}$. Even though they lie outside the actual investigation regions, there is a fairly good agreement of the curves. Hence, we shall generally assume our simulation results to be consistent.

The consistency of the local observables entails that the mixture behaves like a real one in the investigation regions. Consequently, the slight $z$-dependence of the temperatures must be induced outside these regions. We ascribe it to the spatially dependent average heat transport rates along each thermostated region. To understand their cause, we must take a closer look at how the investigation regions exchange heat with the intermediate regions. We know from the corresponding nonequilibrium one-phase systems that the Ar1 phase has a lower thermal conductivity than the Ar2 one. Its different magnitudes should apply similarly in the corresponding phases of the present nonequilibrium interfacial system. Concurrently, thermal conduction remains the dominant heat transport mechanism, based on the small local com velocities and thus Péclet numbers (see section 4.2.3). Hence, we can expect a spatially dependent heat flux into or out of the thermostated regions. It is higher in the Ar2 phase than in the Ar1 phase of the present nonequilibrium interfacial system. Our thermostating algorithm does, however, not account for such dependencies. It rather manipulates all particle velocities in a certain region by equal measures, i.e. by the same scaling factor and shift summand at a certain time step. The type of a particle or its exact location inside the thermostated region are, for instance, not considered. In the cold thermostated region, lower temperatures will thus on the average establish at those locations where the heat flux is lower, i.e. the Ar1 phase. They will, on the other hand, have on the average higher temperatures in the hot thermostated region. The present heterophasic nonequilibrium interfacial system exhibits this type of behaviour. We shall later see it even worsen if the conductivities differ more between both phases of a system. 


\section{Comparison to the Local Data in the Corresponding Systems}

Remember the dichotomy in our nonequilibrium interfacial system. It tries to establish local equilibria across the interfaces but concurrently tends to separate in $y$-direction due to thermal diffusion (see section 4.2.3). The corresponding equilibrium interfacial and nonequilibrium onephase systems from above constitute limiting cases of these contradictory tendencies. We shall determine here the quantitative influence of the thermocapillary convection and of the thermostating algorithm by comparing the different systems with each other.

At first, we shall compare the results at the centres between the thermostated regions, i.e. at $|y|=2.0 \mathrm{~nm}$, with those in the corresponding equilibrium interfacial system. The temperature $z$-profile constitutes, despite the diverging thermal conductivities of both phases, almost a constant function at such $y$-coordinates (see Figure 38b). Its value includes only the drop in the average system temperature due to the disparity of the temperature jumps at the thermostated regions. Hardly any differences appear in the densities, and thus also the interatomic volume and particle $z$-forces, despite these temperature anomalies (see Figure 38a,c,d).

More significant influences arise between each phase and its corresponding nonequilibrium onephase system. Thus, the temperature $y$-profile is steeper in the Ar1 rich phase and flatter in the Ar2 rich one (see Figure $38 \mathrm{~b}$, Table 8 gives the numbers for the $y$-gradients). Such a behaviour agrees with the above described malfunction of our thermostats due to the different conductivities of both phases (see Table 14). Nevertheless, each of them features a stronger separation in $y$-direction, as can be clearly told from the increased Soret coefficients (see Table 11). We would have expected smaller ones at first sight because the thermocapillary convection should, even if it was weak, sustain a better mixing. Hence, we shall keep an eye on this separation in our subsequent studies to find out more about its influences. It manifests itself also in the steeper overall and partial density $y$ profiles in the Ar1 rich phase (see Figure 38a and Table 13). They change, however, only marginally in the Ar2 rich phase due to the lower temperature $y$-gradient there. Table 8 compares additionally the I-averages of the different interatomic $y$-forces in the phases of the nonequilibrium interfacial and its corresponding nonequilibrium one-phase systems. Only the $y$-forces per minority particle strongly increase in each phase compared to its corresponding nonequilibrium one-phase system, while the ones per majority or per unit volume have approximately the same magnitudes. 
We cannot draw more conclusions from the systems above. The bias from the malfunctioning thermostats is too strong. Nevertheless, they provide already fairly good insights into their characteristic behaviour. Hence, we shall continue to use them and similar ones in chapter $\mathbf{5 . 2}$ for further semiquantitative studies where this bias is unimportant of inevitable. Chapters $\mathbf{5 . 4}$ and $\mathbf{5 . 5}$ describe more advanced quantitative studies of systems which have identical particle parameters for both substances to avoid the malfunction of our thermostats.

\section{Global Observables and their Standard Deviations}

Table 14 lists the global observables and their standard deviations. Although both describe the system behaviour as a whole, we can learn a lot from them. Thus, the standard deviations, in particular, have approximately the same orders as in the corresponding nonequilibrium one-phase systems. Such a behaviour is the more surprising if we remember the existence of the thermocapillary convection and the anomalous temperature $z$-dependence in the nonequilibrium interfacial system. Hence, they can both hardly produce additional numerical and simulation errors. We can tell the same from the global temperature. It drops approximately as much as in the corresponding nonequilibrium one-phase system below the one of the equilibrium interfacial one. Such a drop entails concurrently slightly smaller global pressures and energies.

Let us now take a closer look at the heat flux, $Q$. It lies in between those of the corresponding nonequilibrium one-phase systems. Such a relation seems reasonable since their phases have approximately the same composition but twice the volume of the interfacial system. After all, thermal conduction remains the dominant transport mechanism. We notice, however, upon closer inspection the slightly increased vicinity of the heat flux to the worse heat conducting one of the nonequilibrium one-phase systems. A lower entropy production thus also exists in the nonequilibrium interfacial system than could be expected by superimposing them. We shall check if the same holds also in the systems to come. Such a behaviour would suit the principle of minimum entropy production (see section 2.1.1). 


\subsection{Examination of the Parameters and Simplifications in the Heterophasic Systems}

Molecular dynamics simulations involve, as detailed in chapter 3.2, a number of simplifications and approximations. It is the main purpose of this chapter to estimate, as far as possible, their influence on the simulation results. To do so, we shall compare in the sections below the first reference systems from chapter $\mathbf{5 . 1}$ with only slightly altered systems. They have different particle parameter values, cut-off radii, or use a modified thermostating algorithm. The comparison concurrently reveals first influences on the thermocapillary effect.

Apart from the thermostating, the truncation of the interatomic forces beyond a certain cut-off radius counts among the trickier technical simplifications. We manipulated it in additional simulations, to find out how much the system behaviour has converged (see section 5.2.1). In doing so, we disregarded possible interdependencies between the force truncation and the thermostating procedure. It may rather suffice to estimate the influence of the cut-off radius by using always the same thermostats. The results confirm our chosen radius of $r_{c}=1.0 \mathrm{~nm}$ as appropriate. Concurrently, they reflect the influence of the LB mixing parameter, $\xi$, or of the average system temperature. A significant improvement of the anomalous temperature $z$-dependence does, however, not occur.

Given the missing influence of the force-truncation on the anomalous temperature $z$-dependence, we confirmed our likely explanation for it by manipulating the mass of the individual ArB particles (see section 5.2.2). Since the static observables generally do not depend on the particle mass, their distributions remain unaffected by it in equilibrium interfacial systems (see section 3.1). This independence ceases, however, in nonequilibrium systems. They involve transport processes, described by static and, in addition, by dynamic observables. The nonequilibrium one-phase systems display clearly the response in the thermal conductivity. It increases specifically in an ArB rich mixture strongly with its particle mass. We can expect similar responses in the corresponding phases of the nonequilibrium interfacial system. Their stronger diverging conductivity thus likely worsens the anomalous temperature $z$-dependence in agreement with our explanation. This will turn out to be the case. 
If the thermal conductivities of both phases differ only little, the system behaviour approximates reality to a large extent, as demonstrated previously for the first Ar1Ar2 nonequilibrium interfacial system. Aiming at improving this approximation, we implemented another (loose) version of our thermostats to see whether the overmodulation of the thermostated temperatures (see section 5.1) contributes to the temperature jumps or even to the anomalous temperature $z$-dependence. Such a contribution does, as it will turn out in the discussion of the results in section 5.2.3, not occur in the tuning the additional parameters of the loose thermostats. The heat fluxes into and out of the thermostated regions have less fluctuations, but at the price of stronger ones in the thermostated temperatures.

We could imagine even more sophisticated thermostating procedures, for instance, doubling the number of thermostats to four while maintaining the two thermostated regions and the allocation formula: Then each thermostat would be concerned only with the particles of a certain substance in a certain thermostated region. Developing such procedures lies, however, outside the focus of our work. Instead, we sticked with the strict thermostats in the subsequent simulations for the sake of comparability. There is another, simpler measure to abandon the anomalous temperature $z$ dependence. We equalised the thermal conductivity of both phases by choosing identical LJ parameters for all interactions of like particles (see chapter 5.3). The absence of a tangible archetype makes such a mixture rather unorthodox. Nevertheless, it should behave realistic in simulations since its description bases on LJ potentials. They are physically meaningful irrespective of their precise curves. In order to improve the comparability of our results, we chose LJ parameter values, that are frequently used in the literature, when changing them anyway. The thus obtained homophasic Ar5Ar5 system also behaves realistic. Concurrently, its differences from the Ar1Ar2 system reveal the influence of the LJ parameters.

Note, all discussions in chapter $\mathbf{5 . 4}$ concern the results in homophasic nonequilibrium interfacial systems of the type described above. We shall vary systematically those of their parameters that have a physical correspondence. In doings so, the Ar5Ar5 system from chapter $\mathbf{5 . 3}$ frequently serves us as a reference. 


\subsubsection{Influence of the Cut-Off Radius on the Local Observables}

We altered the cut-off radius in several simulations of the first heterophasic nonequilibrium interfacial and its corresponding systems to find out more about the influence of the truncation of the interatomic potentials and forces. As explained in section 4.1.2, they are, by default, ignored for particle distances of more than $r_{c}=1.0 \mathrm{~nm}$ to reduce computational efforts. Radii of $r_{c}=0.6 \mathrm{~nm}$ and $r_{c}=1.2 \mathrm{~nm}$ were used additionally for comparison. They entail, approximately, a halving and a doubling of the simulation times. In the discussions of the results below, we will be able confirm once again that our initially chosen cut-off radius of $r_{c}=1.0 \mathrm{~nm}$ is a suitable compromise between a proper treatment of the longer-ranged interatomic forces and the computing efforts. On the other hand, the results are also of interest because the systems respond qualitatively to the manipulations of the cut-off radius in the same way as to changes of the mixing parameter $\xi$ in the LorentzBerthelot mixing rule or of the global temperature. Thus, an expansion of the cut-off radius, for instance, will turn out to be in some sense equivalent to a reduction of the mixing parameter $\xi$ or the global temperature.

\section{Local Observables}

The temperatures at $|y|=2.0 \mathrm{~nm}$ as well as the temperature $y$-gradients exhibit similar distributions in all nonequilibrium systems, irrespective of the cut-off radius used (see Figure 39b). We can thus assume that their removal from equilibrium is also inside the thermostated regions similar enough to provide a common basis of comparison. Straightforward comparisons of the results in the different simulations thus make sense to learn about the influence of the cut-off radius on the system behaviour.

The partial densities are more evenly distributed in $z$-direction in the nonequilibrium interfacial system with the smaller cut-off radius of $r_{c}=0.6 \mathrm{~nm}$ (see Figure 39a). This distribution is accompanied by wider interfaces (see Tables 10-11) and weaker mean interatomic particle $z$-forces (see Figure 39e). Moreover, we can tell from the normal stresses in the corresponding equilibrium interfacial system (given in the same tables) that the magnitude of the interfacial tension decreases. Consequently, the local states in the nonequilibrium interfacial system are shifted closer to the 
upper critical solution point. Concurrently, both substances separate more strongly in $y$-direction within each phase, with more pronounced variations of the partial density $y$-gradients in the interfacial regions (see Figure 39a and Table 13). Such an increased thermal separation makes sense because the mutual solubility of both substances becomes more temperature dependent, when approaching the critical solution point. Hence, the partial density $y$-gradients in each phase are steeper than in the corresponding nonequilibrium one-phase system (also given in Table 13). Moreover, both interatomic particle $y$-forces differ, according to their relation with the density $y$ gradients, more within each phase than in its corresponding one-phase system (see Table 8).

Given the smaller cut-off radius, the overall densities exhibit, different from the partial densities, more even distributions in the $y$ - and $z$-directions (see Figure 39a). This distribution manifests itself also in the lower interatomic volume $y$ - and $z$-forces (see Figure 39d, and Table 8). The thermocapillary convection weakens, but its characteristic pattern remains unchanged (see Figure $39 \mathrm{c})$. We can tell the reduced intensity e.g. from the smaller maximum com velocity components (see Table 11). Its reduction is in agreement with the flatter $y$-gradient of the normal stresses, $S_{z z}$, (see Figure 39f and Table 11), which serves as a measure for the strength of the thermocapillary effect. Moreover, the general level of all normal stresses decreases since they include fewer longranged attractive interatomic forces in the usage of the smaller radius (see Table 6).

All previously described responses to the change of cut-off radius (see all tables and figures mentioned above) invert, when expanding it to $r_{c}=1.2 \mathrm{~nm}$ (which, by the way, doubles the CPU time). They are, however, much less pronounced than the ones observed for the reduction of the radius to $r_{c}=0.6 \mathrm{~nm}$ and can hardly be extracted from the fluctuations in many cases. Relatively large responses are still found in the normal stresses, but even they exhibit a clear convergence. Since the qualitative differences between the various simulations are more important to us than the absolute values of the observables, we kept the initially chosen cut-off radius of $r_{c}=1.0 \mathrm{~nm}$. It is a good compromise between a more complete inclusion of the longer-raged interactions and the computational efforts. We thus used this value in all subsequent simulations. The standard deviations of the local observables converge together with them (see as well in all the tables and figures mentioned above). 


\section{Global Observables}

Table 14 lists all global observables determined in the simulations with different cut-off radii. The global pressures and energies converge, similar to the negative local normal stresses, to lower limits when we increase the cut-off radius. This convergence is presumably since more, but increasingly weaker, attractive long-range interactions are considered. We cannot tell about the changes in the convergence of the heat supply and removal they disappear in the noise of the simulations. Nevertheless, these fluxes always lie below those in the corresponding nonequilibrium one-phase systems, which confirms again that convective transport remains negligible. The global temperatures remain are unaffected by the cut-off radius since they do not relate directly to the truncation of the particle interactions. Hardly any changes occur in the global standard deviations, as in the local ones described before. 


\subsubsection{Influence of the Thermal Conductivity on the Thermostating Artefacts}

The molecular transport phenomena, i.e. essentially heat conduction, diffusion, and thermal diffusion, dominate the convective transport in our nonequilibrium interfacial systems. We shall compare below the first such system (studied in chapter 5.1) to ones with the altered mass values of $m_{A r 3}=3.995 \mathrm{amu}$ or $m_{A r 4}=399.5 \mathrm{amu}$ for the second substance, generally called ArB. The LennardJones and all other system parameters maintained their numerical values in the alterations. Since the transport phenomena respond strongly to the particle mass we can learn about their interplay as part the system behaviour. Thus, the intensity of the thermocapillary convection will turn out to increase, the smaller the viscosity of the mixture, i.e. essentially the smaller its particle mass, is. Nevertheless, an even more intense convection would have to occur until the molecular transport mechanisms loose their dominant role. Moreover, we shall see that the masses, $m_{A r B}$, do not necessarily correlate in a simple way with the strength of the thermocapillary convection.

As to the studies of the molecular transport phenomena, the anomalous temperature $z$-dependence in the heterophasic nonequilibrium interfacial systems must be additionally taken into consideration. We shall thus look at the corresponding nonequilibrium one-phase systems to study how each phase responds to the change of the ArB particle masses without this artefact. In particular the conductivities will be seen to depend on the particle masses in such a way that our explanation for this anomalous temperature $z$-dependence (see section 5.1.3) is confirmed. However, before we look at the nonequilibrium one-phase systems, we shall consider, as a precaution, their corresponding equilibrium interfacial ones. They allow us to verify our simulation code by confirming in them the independence of results from the particle mass.

\section{Comparison of the Equilibrium Interfacial Systems}

All static observables are, as they should be, similarly distributed in the equilibrium interfacial systems with different masses. Compare, for instance, the densities in Figure 40a. The interatomic volume or particle forces have similar distributions as well (see Figure 40d,e). We must, of course, disregard in the comparisons the statistical noise and, as far as the quasi-intensive observables are concerned, also the slightly deviating locations of the subvolumes with respect to the interfaces. 
Nevertheless, the normal stresses are slightly shifted, by about $0.4 \mathrm{MPa}$, in the system with the lightest ArB particles. Figure 40f and Table 6 show that this is due to a larger interatomic contribution. Minor temperature differences occur, moreover, even within each of the systems with the altered ArB particle masses (see Figure 40b and Table 8). Thus, the temperature of the phase containing mainly the heavier particles always slightly exceed the one of the other phase, i.e.

$\varnothing[\mu(T): \operatorname{Ar} 1]>\varnothing[\mu(T): \operatorname{Ar} 3]$ in N-Ar1Ar3-0.6-1.0-3346-3524-4.74x8.00x9.40-100-140

and

$\varnothing[\mu(T): \operatorname{Ar} 4]>\varnothing[\mu(T): \operatorname{Ar} 1]$ in N-Ar1Ar4-0.6-1.0-3346-3524-4.74x8.00x9.40-100-140.

Nevertheless, the system averaged temperature $\varnothing[\mu(\mathrm{T}): \mathrm{S}]$ (see Table 6) lies close to its set value of 120K. We can neglect these differences since they are so small that they hardly manifest themselves in the other system behaviour. Let us still comment briefly on their cause. They might result from differently severe numerical and simulation errors that depend on the particle mass. If they come on the average with spatially dependent energy losses or gains, the thermostating algorithm may produce the temperature differences through a similar mechanism as explained in section 5.1.3. These gains or losses, if they exist at all, will be very small compared to the heat fluxes. We shall discuss them further below. Note that above-mentioned numerical and simulation errors could also explain the found pressure shift.

Most local standard deviations (see Figure 40a-d and Table 8) are similarly distributed in the present equilibrium interfacial systems, even though they do, strictly speaking, not count among the static observables. Only the com velocity standard deviations, in particular the ones in the ArB rich phases, differ significantly, while still closely approximated by the estimation formula, equation $(8-4)$ :

$$
\begin{aligned}
& \left.\phi \mid \sigma\left(v_{y}\right): A r 3\right]=\phi\left[\sigma\left(v_{z}\right): A r 3\right] \approx \\
& \approx \sqrt{\frac{1.38 \cdot 10^{-23} \frac{\mathrm{J}}{\mathrm{K}} \cdot 119.8 \mathrm{~K}}{4.74 \mathrm{~nm} \cdot 0.80 \mathrm{~nm} \cdot 0.26 \mathrm{~nm} \cdot\left(0.06 \mathrm{~nm}^{-3} \cdot 6.634 \cdot 10^{-26} \mathrm{~kg}+19.89 \mathrm{~nm}^{-3} \cdot 0.6634 \cdot 10^{-26} \mathrm{~kg}\right)}}= \\
& =111.1 \frac{\mathrm{m}}{\mathrm{s}} \\
& \left.\phi \mid \sigma\left(v_{y}\right): A r 4\right]=\phi\left[\sigma\left(v_{z}\right): A r 4\right] \approx \\
& \approx \sqrt{\frac{1.38 \cdot 10^{-23} \frac{\mathrm{J}}{\mathrm{K}} \cdot 120.2 \mathrm{~K}}{4.74 \mathrm{~nm} \cdot 0.80 \mathrm{~nm} \cdot 0.26 \mathrm{~nm} \cdot\left(0.06 \mathrm{~nm}^{-3} \cdot 6.634 \cdot 10^{-26} \mathrm{~kg}+19.89 \mathrm{~nm}^{-3} \cdot 66.34 \cdot 10^{-26} \mathrm{~kg}\right)}}= \\
& =11.3 \frac{\mathrm{m}}{\mathrm{s}}
\end{aligned}
$$


We shall, therefore, consider their mass dependence as a physical fact.

Besides the local static observables, the global ones (see Table 14) also strongly resemble each other in the different equilibrium interfacial systems. Take, for instance, the global energies. The abovementioned slight shift of the normal stresses in the system with the lighter Ar3 particles manifests itself also in the global pressure. Its values in the other systems are, however, very similar. The global temperatures approximate their set value of $120 \mathrm{~K}$ in all systems while the heat in- or outflux (given in as well in Table 14) is approximately zero.

\section{Comparison of the Nonequilibrium One-Phase Systems}

We simulated the phases of the equilibrium interfacial systems separately in nonequilibrium onephase systems under the default volume and temperature constraints. In doing so, we can learn about the separate influence of the particle masses on the molecular transport phenomena, i.e. without having to worry about the role of the thermocapillary convection or the artefacts due to the thermostating as in the nonequilibrium interfacial systems. The identity of the volume and temperature constraints does, however, not necessarily entail yet that all systems are similarly removed from equilibrium. Depending on their composition or their ArB particle masses, temperature jumps of different magnitudes could occur at the thermostated regions. Hence, we shall first check the similarity of the temperature distributions using their temperature M-averages and $y$ gradients (see Tables 9 and 13). They hardly differ between systems rich in the same, i.e. either the first or the second, component. Somewhat stronger differences exist between the ArA and the ArB rich systems, but they are still small enough so that we can proceed to study the influence of the ArB particle masses by straightforward comparison of the other observables.

In the comparisons of the other observables we shall, moreover, neglect the differences between the numbers of particles in the nonequilibrium one-phase systems that are rich in the same, i.e. the first or the second, component. In fact, these numbers should be identical since they were computed from the partial densities in the phases of the equilibrium interfacial systems above, that differ only in their particle masses. Minor deviations between such systems are, however, unavoidable. Even though these deviations propagate throughout the evaluations, they amount to only a few particles and can thus be ignored safely. 
The partial densities respond to the alterations of the ArB particle masses only slightly in absolute terms, but significantly as far as the thermal diffusion is concerned. Remember from section 5.1.2 that the minority particles concentrate at the hot and the majority particles at the cold region of the one-phase systems with identical particle masses. This separation decreases, or even inverts, in the systems with the changed ArB masses if the heavier particles are outnumbered (N-Ar1Ar3-0.6-1.0-

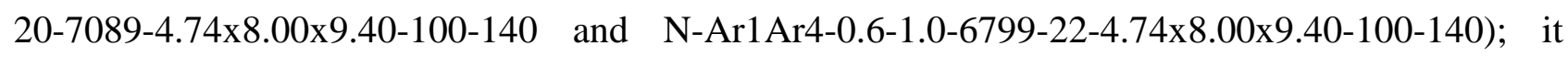
strongly increases if they are in the majority (N-Ar1Ar3-0.6-1.0-6797-22-4.74x8.00x9.40-100-140 and N-Ar1Ar4-0.6-1.0-19-7088-4.74x8.00x9.40-100-140). We can tell so by comparing the absolute differences between the partial densities in each system (see Figure 40a and Table 13). The Soret coefficients in Table 11 show the changes in the separation even more clearly. They are in agreement with previous studies of the thermal diffusion in equimolar mixtures of simple liquids, where the heavier component always tends to move towards the cold region (see section 4.2.2). The interatomic $y$-forces per particle (see Table 8) differ between the systems, that are rich in the same substance, according to the strength of the thermal diffusion in them. Concurrently, there is a sign of these forces for the heavier minority particles. The overall density $y$-profiles (see Figure 40a) and the interatomic volume $y$-forces (see Table 8) are hardly affected. Only a slight steepening of the $y$ profiles occurs in systems where both substances move towards the cold region. Their movement is accompanied by marginally stronger volume $y$-forces. We shall check further below to what extent the thermal diffusion found in the nonequilibrium one-phase systems appears also into the corresponding nonequilibrium interfacial systems.

As far as the other local observables and the standard deviations are concerned, they respond to the alterations of the ArB masses mostly as in the corresponding equilibrium interfacial systems above (see Figure 40a-d). We saw clearly shifted normal stresses in the one of them with the lighter Ar3 particles. Such a shift can be identified as well in its corresponding nonequilibrium one-phase system rich in the same particles (see Table 7). Hence, their mutual collisions likely produce the numerical and simulation errors conjectured above.

The values of the global pressures or temperatures (see Table 14) strongly resemble each other in the different nonequilibrium one-phase systems. The global temperatures are somewhat below the ones in the corresponding equilibrium interfacial systems due to the different magnitudes and extensions of the temperature jumps. We established already above that they react mainly to the system composition but hardly to the alterations of the ArB particle masses. Accordingly, the 
nonequilibrium one-phase systems rich in the same, i.e. the first or the second, substance have even more similar global temperatures. The same holds also for the global energies (given as well in Table 14). The global pressures, on the other hand, continue to exceed somewhat the ones in the corresponding equilibrium interfacial systems. This results largely from their lower local pressures in the interfacial regions. If they are again disregarded, however, by spatially averaging the negative normal stresses only within each phase of an equilibrium interfacial system, its so-obtained pressures, $\varnothing[\Pi: \mathrm{ArA}]$ and $\varnothing[\Pi: \mathrm{ArB}]$, approximate the global ones in the corresponding nonequilibrium one-phase systems even better. Being strongly inversely related to the ArB particle masses in the ArB rich systems, the heat fluxes have approximately the same magnitude in the ArA rich ones. We give these heat fluxes as well in Table 14 and derive thermal conductivities from them (see Table 11). They shall be assumed below to apply roughly as well in the applicable phases of the corresponding nonequilibrium interfacial systems.

\section{Comparison of the Nonequilibrium Interfacial Systems}

Strong alterations of the original anomalous temperature $z$-dependence accompany the manipulations of the ArB masses in the heterophasic nonequilibrium interfacial systems (see Figure 40a,b as well as Table 13). Thus, the temperature $y$-gradient in the Ar1 phase is already steeper than in the other phase of the original Ar1Ar2 system. When the mass of the second component, generally called $\mathrm{ArB}$, is reduced to $m_{A r 3}=3.995 \mathrm{amu}$, the $y$-gradient in the ArB phase increases much stronger than the one in the Ar1 phase. When this mass is increased to $m_{A r 4}=399.5 \mathrm{amu}$ instead, the temperature $y$-gradient in the ArB phase drops much more than the one in the Ar1 phase. As explained already for the Ar1Ar2 reference systems above (see section 5.1.3), both alterations correlate with the changes of the thermal conductivities. We do not determine them here, but we can assume, for the same reasons as in these reference systems, that the thermal conductivities in the phases of either manipulated nonequilibrium interfacial system also resemble those in their corresponding nonequilibrium one-phase systems, i.e. the Ar3 phase has a much larger and the Ar4 phase a much smaller thermal conductivity than the Ar1 or the Ar2 phase. Concurrently, the heat transport in the changed nonequilibrium interfacial systems must continue to be dominated by thermal conduction as can be told from the $z$-independence of the local observables remote from the interfaces (discussed below). Hence, the temperature $y$-gradient in the Ar1 phase is steeper or flatter than in the other one, depending on whether its local heat transport rates are smaller or larger. Our above explanation of the anomalous temperature $z$-dependence in the heterophasic nonequilibrium interfacial systems is thus confirmed once again. 
Although the anomalous temperature $z$-dependence worsens in the modified nonequilibrium systems, the density $z$-profiles at $|y|=2.0 \mathrm{~nm}$ hardly differ (see Figure 40a). Moreover, they still converge away from the interfaces (i.e. at all other absolute $y$-coordinates), which demonstrates that convective transport continues to be negligible. The partial densities spread more unevenly in $y$ - and $z$-direction. Thus, each of the manipulated systems exhibits a larger absolute difference between both partial density $y$-gradients in the phase where the temperature $y$-gradient steepens and a smaller one in the other phase (see Figure 40a,b and Table 13). Concurrently, stronger variations of the partial densities exist in the interfacial regions while their widths remain almost unaffected (see Table 11). In order to be able to compare the separation of the substances within the phases, we must look at their Soret coefficients (given as well int in Table 11). They incorporate also the different temperature $y$-gradients. As in the case of the Ar1Ar2 systems above (see section 5.1.3), the Soret coefficient in a phase of the modified nonequilibrium interfacial systems exceeds the one in its corresponding nonequilibrium one-phase system. Surprisingly, the Soret coefficient of the ArB phase is, however, always positive, irrespective of the mass of the ArB particles. Such a behaviour contradicts the one observed in the corresponding nonequilibrium one-phase systems above, where the heavier particles tend to move towards the cold region. Apparently, the tendency to establish local equilibria across the interfaces dominates the one to segregate along the temperature gradient (thermal diffusion) in the manipulated nonequilibrium interfacial systems. Our thought experiment from section 4.2.3 postulated a competition between both tendencies. We shall discuss it in more detail later in the homophasic systems (see chapter 5.4).

The interatomic volume and particle forces (see Figure 40d,e) are similarly distributed as in the Ar1Ar2 nonequilibrium interfacial system. Their noise is too large to check the interfacial variations, but the I-averaged $y$-components of these forces add again approximately to zero with their related kinetic forces (see Table 8). The standard deviations of the local observables described up to here are given together with them in all the figures and tables referenced above. These standard deviations respond to the alterations of the ArB masses in the same way as in the corresponding equilibrium interfacial systems above.

As far as the thermocapillary convection is concerned, its general pattern hardly changes upon manipulating the ArB mass (see Figure 40c), even though the viscosities are known to be sensitive to it. We did not determine these viscosities explicitly but their relative magnitudes can be estimated from the velocity standard deviations. Moreover, we know from the theory of Chapman and Enskog 
[chapman1970] that the viscosity of a monatomic gas increases with its particle mass. A similar mass dependence should apply, at least qualitatively, in the phases considered here since their states lie relatively close to the critical points of the pure substances. Hence, we can assume similar viscosities for all Ar1 rich phases. The viscosities of the ArB rich phases, on the other hand, increase for the lowered and decrease for the increased ArB masses while having approximately the same value as in the Ar1 rich phase in the system with identical particle masses. Despite these differences, the intensity of the thermocapillary convection changes, as stated above, in a similar way in both phases, namely inversely to the viscosity of the ArB rich phase.

We encounter another important aspect in the behaviour of the modified nonequilibrium interfacial systems if we relate the intensity $\left(\operatorname{MAX}\left(\left|v_{y}\right|\right)\right)$ of the thermocapillary convection to the pressure $y$ gradient $\partial \Pi / \partial y$ in the phases (see Table 13). It is, as explained in section 4.2.3, the driving force of the backward flows in the phases. According to our expectations, the pressure gradient $\partial \Pi / \partial y$ is the stronger the larger the viscosity in the ArB-rich phase is, while the convection becomes less intense. In the subsequent studies we shall keep an eye on these properties to find out more about their relations.

In spite of the differences in the local observables between the various nonequilibrium interfacial systems, they all have surprisingly similar global temperatures, pressures, and energies, including their standard deviations (see Table 14). They also approximate the ones in the corresponding equilibrium interfacial systems. The temperature drop is almost unaffected by the ArB mass. The previously described disparity of the temperature jumps at the thermostated regions rather occurs regardless of how strong the temperatures vary with $z$. Slightly lower pressures and energies are found according to the magnitudes of the drops. The average heat flux, and thus also the entropy production, change in the same way as in the corresponding ArB rich nonequilibrium one-phase systems, i.e. both properties increase for the lower ArB mass and decrease for the higher one. Nevertheless, they always remain below their values in these systems, as demonstrated previously for the Ar1Ar2 mixture in section 5.1.3. Hence, thermal conduction is confirmed to dominate over convective heat transport also in the modified nonequilibrium interfacial systems. The standard deviations of the heat fluxes lie approximately at the centre between their values in the same regions of the corresponding nonequilibrium interfacial one-phase systems. 


\subsubsection{Influence of the Overmodulation by the Thermostats on the System Behaviour}

In the NEMD simulations discussed up to here we used so-called "strict" thermostats, see section 4.1.4. They adjust the kinetic energies of the particles so as to establish at every time step exactly the intended temperatures in the "thermostated regions". As a result, overly large fluctuations appear in the rates of heat supply, $Q_{H}$, and removal, $Q_{C}$. They can even invert from time to time due to the "overmodulation" described in section 5.1.2. We thus resimulated the first heterophasic nonequilibrium interfacial system, N-Ar1Ar2-0.6-1.0-3346-3524-4.74x8.00x9.40-100-140-r1, using "loose" thermostats instead, N-Ar1 Ar2-0.6-1.0-3346-3524-4.74x8.00x9.40-100-140-1s. They adjust the kinetic energies following the same allocation formula as the strict version, but stepwise by smaller amounts. The results will be compared below with the ones for the strict thermostat in order to estimate the error from the overmodulation. It will turn out to decrease significantly with the loose version, but at the price of stronger fluctuations in the instantaneous temperatures. Moreover, the reduction of the overmodulation has, contrary to our expectations, slightly adverse effects on the temperature jumps near the thermostated regions. Hence, we shall at the end continue to use the strict version.

\section{Tuning of the Loose Thermostats}

Before the loose thermostats could be employed, we had to pick suitable weights for their proportional and integral terms, see section 4.1.4 for their definitions. The thermostats were tuned in several simulations of the same system by a straightforward procedure described below. We resimulated the first heterophasic nonequilibrium interfacial system in several runs, setting the thermostats to different proportional or integral gains in each run. Table 14 lists the gains together with the resulting thermostated temperatures as well as the heating or cooling rates of the thermostats.

We first gradually reduced the proportional gain from its recommended value of $k_{P}=0.0125$ for a Berendsen thermostat (see section 4.1.4) while the integral gain was held constant at $k_{l}=0$. The results show clearly that, with these settings, the loose thermostats cannot provide the necessary 
capacity to maintain the intended temperatures in the thermostated regions. On the contrary, the difference in temperature between them, and thus the heat flux through the system, decreases with decreasing proportional gain $k_{P}$. Concurrently, the standard deviations of these heat fluxes decrease, while the ones of the thermostated temperatures increase only slightly.

We then analysed the role of the integral gain in further production runs where it was gradually varied between zero and unity while maintaining a constant proportional gain of $k_{P}=0.0125$. The thermostated temperatures approximate the set values surprisingly well, irrespective of the chosen integral gain. Moreover, heat fluxes, similar to the ones found with strict thermostats, exist in the system. The standard deviations of the heat fluxes and of the thermostated temperatures increase with the integral gain $k_{I}$.

We can, in principle, combine the above-established separate influences of the different gains. Suitably chosen values provide, when combined, a much more balanced ratio between the fluctuations in the heat fluxes and those in the thermostated temperatures than the strict thermostats. As far as the present nonequilibrium interfacial system is concerned, the combination of a proportional gain of $k_{P}=0.001$ with an integral one of $k_{I}=0.0001$ seems to be a good choice. It produces much smaller fluctuations in the heat fluxes than the strict thermostats, without overly affecting the temperatures in the thermostated regions.

\section{Comparison of the Simulation Results for the Tuned Loose and the Strict Thermostats}

We significantly reduced the fluctuations in the heat supply and removal by using the loose thermostats (see Table 14). This is at the price of stronger fluctuations in the thermostated temperatures. The general system behaviour is, however, hardly affected: We are particularly interested in the distribution of the temperatures. The temperature jumps at the hot thermostated region increase by approximately the same amount as the ones at the cold thermostated region decrease. Accordingly, a temperature drop of about $0.4 \mathrm{~K}$ exists at both regions even though the temperature $y$-gradients remain almost unaffected (see Figure $41 \mathrm{~b}$ as well as Tables 8 and 13). No change can be observed in the anomalous temperature $z$-dependence. Contrary to our expectations, the loose thermostats do not produce a more realistic temperature distribution. Instead, they even slightly exacerbate the disparity between the temperature jumps at the hot and the cold thermostated 
region. We shall thus continue to use the strict thermostats for the future nonequilibrium simulations.

For the sake of completeness we shall take a brief look at some other observables, here: The marginal differences between both simulations result, if not from the noise, largely from the drops in the temperatures (see Figure 41 as well as Tables 6-14). Thus, the negative local normal stresses as well as all local standard deviations decrease, as in previous simulations, and so do the global temperatures, pressures, and energies decrease. A clear response of the densities, the com velocities, the different interatomic forces, as well as the heat supply and removal to the temperature drop cannot be recognised in the noise of the simulations. 


\subsection{Homophasic Systems with a Better Approximation of Reality}

(first homophasic nonequilibrium interfacial and its corresponding systems)

The Ar1Ar2 nonequilibrium reference systems from chapter 5.1 exhibited only minor anomalies. They can, however, easily deteriorate in certain variations of the simulation parameters. Think for instance of the increased temperature $z$-dependence that resulted from the manipulations of the ArB particle mass (see section 5.2.2). Such unphysical concomitants complicate more advanced studies of the system behaviour. On the other hand, several adjustments to severe technical simplifications failed to eliminate these anomalies in chapter 5.2. We could imagine more sophisticated simplifications, for instance, doubling the number of thermostats to four, while maintaining the two thermostated regions and the allocation formula: Then each thermostat would be concerned only with the particles of a certain substance in a certain thermostated region. Refining the NEMD simulation methods lies, however, outside the focus of our work. Instead, we sticked with the strict thermostats below for the sake of comparability.

There is another simpler measure to abandon the anomalous temperature $z$-dependence. We keep the mixtures "symmetric", i.e. we always give both components identical particle masses, $m_{A r A}=m_{A r B}$, and identical LJ parameter values for the interactions of like particles, $\sigma_{A r A A r A}=\sigma_{A r B A r B}$ and $\varepsilon_{A r A A r A}=\varepsilon_{A r B A r B}$. If the cross terms for the interactions of unlike particles, i.e. $\sigma_{A r A A r B}$ and $\varepsilon_{A r A A r B}$ and/or the mixing parameter $\xi$, and the thermodynamic conditions are such that the systems separate into two liquid phases, both will have identical properties. This leads e.g. to the advantageous effect that, due to the equal thermal conductivities on both sides of the interfaces, distortions in the temperature $z$-profiles are minimised. Other biases, such as, for instance, the temperature jumps at the thermostated regions, persist however. They must still be accounted for in the interpretation of the simulation results. We shall generally call such interfacial systems "homophasic", to distinguish them from the "heterophasic" ones studied before. The absence of a tangible archetype makes these homophasic systems rather unorthodox. Nevertheless, they should behave realistic in a simulation since their description bases on LJ potentials. They are physically meaningful irrespective of their precise curves. In order to improve the comparability of our results, we chose LJ parameter values, that are frequently used in the literature, when changing them anyway. Moreover, their influence on the system behaviour manifests itself in comparison to the Ar1Ar2 system. 
Note, all results discussed in chapter $\mathbf{5 . 4}$ involve homophasic nonequilibrium interfacial systems of the type described above. We systematically varied their parameters, many of them having physical correspondence. In the variations, the Ar5Ar5 nonequilibrium interfacial and its corresponding systems frequently served us as a reference. Their function as a reference made us discuss their behaviour here in a separate chapter.

Since we postulated the identity of both components, a new set of particle parameters suggested itself. Michels et al. determined LJ parameter values for pure Argon [michels1949] that are frequently used in the literature. We picked them also for both components in our Ar5Ar5 reference systems (see Table 3) to provide a better comparability of the results with literature data. The mixing parameters $\xi=0.6$ and $\eta=1.0$ (see Table 4) as well as the default system constraints (see Table 5) were kept from the previous Ar1Ar2 reference systems for convenience. Only the particle numbers of the corresponding nonequilibrium one-phase systems had to be redetermined, of course. Refer to section 4.1.2 for the general role of the different particle parameter values.

We shall compare in the sections below the simulation results of the different Ar1Ar2 and Ar5Ar5 reference systems. No qualitative differences will be found, except that, as was mentioned above, the anomalous temperature $z$-dependence tends to disappear when the phases are equal. In this respect, we can consider the somewhat artificial system of an interface between two equal phases as even more realistic. The influence of the LJ parameter values on the quantitative system behaviour will turn out diverse. Thus, they produce a generally increased mutual miscibility of both components than in the Ar1Ar2 mixture. Moreover, a, in terms of the Soret coefficient, stronger thermal diffusion exists in the corresponding nonequilibrium one-phase systems. Both changes manifest themselves also in the corresponding nonequilibrium interfacial system. It exhibits additionally more intense thermocapillary flows, accompanied by a steeper pressure gradient in the phases. In all cases convective transport remains negligible. We shall encircle the number in the symbol of the first Ar5 component with a white background and the number of the second one with a black background in order to distinguish them in the comparisons with the Ar1Ar2 systems below. 


\subsubsection{Equilibrium Interfacial Systems}

In the Ar1Ar2 mixture, the interactions between like particles have the same LJ $\sigma$-parameter values as those between unlike particles, $\sigma_{A r 1 A r 1}=\sigma_{A r 2 A r 2}=\sigma_{A r 1 A r 2}$. The same holds also in the Ar5Ar5 mixture except that it features somewhat smaller LJ $\sigma$-parameter values,

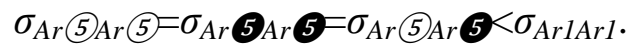

Its $\mathrm{LJ} \varepsilon$-parameter values for the interactions between unlike particles, $\varepsilon_{\operatorname{Ar}(5) \operatorname{Ar}}$, lie closer to those for the interactions between like particles, $\varepsilon_{A r(5) A r(5)}$ and $\varepsilon_{A r} \boldsymbol{\sigma}_{A r} \boldsymbol{( 5}$, than in the Ar1Ar2 mixture. As a result, we would expect a more even density distribution in the equilibrium system, E-Ar5Ar5-0.61.0-3346-3524-4.74x8.00x9.40-120, than in E-Ar1 Ar2-0.6-1.0-3346-3524-4.74x8.00x9.40-120-ber. Also, all the other system properties should occur, according to such a more even density distribution, in a similar way as found in previous studies, e.g. in section 5.2.1. In fact, the simulation results below will confirm our expectations. Thus, we are dealing with less pronounced but therefor wider interfacial variations of the different local observables. Take for instance the above-mentioned densities, the interatomic $z$-forces, the normal stresses lateral to the interfaces, or the local standard deviations (see Figure 42). We had to redetermine the limits of the phases accordingly. Their definition uses a combination of the locations and widths of the interfaces, as explained in section 4.3.5.

In the Ar5Ar5 system, local observables, such as e.g. the densities, the normal stresses lateral to the interfaces, or many local standard deviations, must be symmetrically distributed with respect to the nearest interface. Such a distribution follows from the identical LJ parameter values for the interactions between like particles. The local observables actually exhibit this symmetry to a large extent, when disregarding the statistical noise and the coarse spatial resolution (see Figure 42). Minor asymmetries exist, however. They result presumably from fluctuations. Take for instance the densities (see Figure 42a). In particular, the phase averaged overall and majority component densities in the Ar5 rich phase exceed their values in the Ar5 rich phase. On the other hand, the minority component densities are smaller. These asymmetries disappear, however, in systems with a larger $z$-dimension (see Table 8). Hence, we might be dealing with a finite size effect here. It manifests itself more clearly in the smaller system E-Ar5Ar5-0.6-1.0-3346-3524-4.74x8.00x9.40120 because its phases both have relatively thin $z$-dimensions. Ar5 is, however, somewhat more abundant than Ar(5) and thus segregates into a slightly wider phase. We shall neglect the only 
minor differences between both phases hereafter by considering them strictly identical. The computed spatial averages (see Tables 18-19 and 23) incorporate their identity in the way described in section 4.3.5.

Most of the local observables differ only little in absolute terms between both systems, if at all. Thus, the kinetic contribution to the normal stresses is of the same magnitude in both the heterophasic and the homophasic systems (see Figure 42f). A clearly more significant difference exists, however, in the interatomic contribution. Its value decreases by more than $10 \mathrm{MPa}$ throughout the entire homophasic system. Such a shift is in agreement with the smaller LJ $\sigma$ - and $\varepsilon$ parameter values of the Ar5Ar5 mixture. They increase the importance of the repulsive pair interactions for the behaviour of the Ar5Ar5 system. If the attractive interactions were more important instead, the interatomic contribution would increase, because more terms in its defining equation (see section 4.3.2) would be negative. Virtually no differences can be recognised between both systems in terms of their local temperatures and com velocities.

In analogy with the quasi-intensive observables themselves, their standard deviations are more evenly distributed in the Ar5Ar5 system. Moreover, they take almost identical values in both phases. The previously established relationships between the local observables and their standard deviations remain valid. As to the global observables (see Table 14), the energy and pressure increase in the Ar5Ar5 system. Their increase is in agreement with the higher relevance of the repulsive pair interactions. We explained it above for the interatomic contribution to the normal stresses. The global temperature has approximately the intended $120 \mathrm{~K}$ while the heat flux lies close to zero. 


\subsubsection{Nonequilibrium One-Phase Systems}

As explained before, we reproduced the nonequilibrium behaviour of each phase separately in a, as we call it, nonequilibrium one-phase system. The two ones, N-Ar1Ar2-0.6-1.0-6795-244.74x8.00x9.40-100-140 and N-Ar1Ar2-0.6-1.0-20-7091-4.74x8.00x9.40-100-140, for instance, each relate to a certain phase in the Ar1Ar2 equilibrium interfacial system, E-Ar1Ar2-0.6-1.0-33463524-4.74x8.00x9.40-120-ber. As seen in the previous section, both phases, which should ideally be identical, strongly resemble each other in the simulation of the Ar5Ar5 equilibrium interfacial system. In its case, we could thus spare simulating two corresponding non-equilibrium one-phase systems. A single one of their kind reproduces rather the nonequilibrium behaviour of both phases, when taking into account their symmetry with respect to the first and second component. As a result, we shall use in the discussion below the terms "majority" and "minority" component only. The minority component is, for instance, $\operatorname{Ar} 5$ in an $\operatorname{Ar} 5$ rich phase or $\operatorname{Ar} 5$ in an $\operatorname{Ar} 5$ rich one. The component mentioned first in the notation of the nonequilibrium one-phase systems will always be the majority component, i.e. Ar(5) in the present Ar5Ar5 system, N-Ar5Ar5-0.6-1.0-6744-2234.74x8.00x9.40-100-140. We derived its particle numbers from the averaged partial densities over both phases as explained in chapter 4.1.

The Ar5Ar5 nonequilibrium one-phase system has, in comparison to the Ar1Ar2 ones, smaller temperatures at the centres between the thermostated regions $(|y|=2.0 \mathrm{~nm})$, but steeper temperature $y$ gradients (see Figure 42b). Both differences result from the more disparate magnitudes of the temperature jumps at the thermostated regions. The magnitude at the hot region is smaller in the Ar1Ar2 systems while the one at the cold region is comparable. We can tell so by extrapolating the temperature $y$-profiles from the investigation regions to the boundary regions. This greater disparity is, however, still so small that it hardly manifests itself also in the other observables.

All nonequilibrium one-phase systems feature linear variations of the densities about their system averages (see Figure 42a). The overall densities in the Ar5Ar5 nonequilibrium one-phase system lie, in between those in the previous Ar1Ar2 ones. The minority component densities are larger and the majority component ones are smaller. We took particular interest in the spatial dependence of the densities. Their $y$-gradients are clearly steeper in the Ar5Ar5 system. Nevertheless, it has the weakest tendency to separate as we can tell from the small Soret coefficient. Its smallness seems 
reasonable, keeping in mind the greater similarity between the LJ parameter values for the interactions of like and unlike particles.

The interatomic particle $y$-forces on the majority and minority component particles in the Ar5Ar5 nonequilibrium one-phase system differ stronger (see Table 8) than in the Ar1Ar2 ones. This is in agreement with the steeper partial density $y$-gradients in the Ar5Ar5 system and manifests itself also in its increased volume $y$-forces. None of the systems exhibits a spatial dependence of the normal stresses. They and their different contributions rather have similar magnitudes as in the corresponding interfacial system. The local com velocities approximate their globally constrained values of $0 \mathrm{~m} / \mathrm{s}$.

Table 14 lists the global observables for the systems studied here. We mentioned already the greater disparity between the temperature jumps at the thermostated regions that exists in the $\operatorname{Ar} 5 \operatorname{Ar} 5$ system. As a result, its global temperature drops somewhat more than that in the Ar1Ar2 systems below the expectable average system temperature of $120 \mathrm{~K}$. Even though, the pressure of the Ar5Ar5 system approximates the one in its corresponding equilibrium interfacial system more than is the case in the Ar1Ar2 systems. Likewise, the energies differ less between the corresponding Ar5Ar5 systems than between the corresponding Ar1Ar2 ones. This higher degree of approximation is in agreement with the more alike particle numbers (and compositions) in the different Ar5Ar5 systems. Moreover, the interfacial variations of the lateral normal stresses are less pronounced in the corresponding Ar5Ar5 interfacial system. We give in Table 11 the thermal conductivities of the different nonequilibrium one-phase systems discussed here. The one with the Ar5Ar5 mixture transfers heat the worst. There are no irregularities in the local or global standard deviations. 


\subsubsection{Nonequilibrium Interfacial Systems}

The previously described changes between the two equilibrium interfacial systems,

E-Ar5Ar5-0.6-1.0-3346-3524-4.74x8.00x9.40-120

vs. E-Ar1Ar2-0.6-1.0-3346-3524-4.74x8.00x9.40-120-ber,

and between the two nonequilibrium interfacial systems,

N-Ar5Ar5-0.6-1.0-6744-223-4.74x8.00x9.40-100-140

vs. N-Ar1Ar2-0.6-1.0-6795-24-4.74x8.00x9.40-100-140

and N-Ar1 Ar2-0.6-1.0-20-7091-4.74x8.00x9.40-100-140,

occur, in a, sort of, "superimposed way" also between their corresponding nonequilibrium interfacial systems (see Figure 42),

N-Ar5Ar5-0.6-1.0-3346-3524-4.74x8.00x9.40-100-140

vs. N-Ar1Ar2-0.6-1.0-3346-3524-4.74x8.00x9.40-100-140-r1.

Thus, the Ar5Ar5 system features identical local properties on both sides of an interface at the same distance from it. Moreover, the partial densities have a more even distribution in $z$ - but a more uneven one in $y$-direction in the Ar5Ar5 system (see Figure 42a). We shall discuss these findings in more detail below.

To be able to understand the above-described behaviour, we must recall our thought experiment from section 4.2.3. Convective transport was confirmed above to be negligible in the nonequilibrium interfacial systems. Hence, we can expect them to exhibit an intermediate density distribution which reflects both their contradictory tendencies: to separate in $y$-direction due to thermal diffusion and to establish local phase equilibria across the interfaces. The Ar5Ar5 nonequilibrium interfacial system, in fact, exhibits, as the $\operatorname{Ar} 1 \operatorname{Ar} 2$ one before (see section 5.1.3), such an intermediate distribution. Its underlying limiting cases, in which either the first or the second tendency dominates, lie, however, much further apart. We can tell so already from the mutual solubility's in each mixture. They depend in the Ar5Ar5 one much stronger than in the Ar1Ar2 one on the temperature (see Figure 43). These data result from additional NVT simulations of corresponding equilibrium interfacial systems at the applicable temperature and pressure levels. Moreover, we found in the simulations of the corresponding nonequilibrium one-phase systems a weaker thermal expansion and thermal diffusion of the Ar5Ar5 mixture. It thus seems as if the 
wider spread between both limiting cases favours the thermocapillary effect: On the one hand, there are the more intense flows (see Figure 42c). On the other hand, there is a steeper $y$-gradient of the normal stresses perpendicular to the interfaces, $\partial S_{z z} / \partial y$ (see Figure 42f). It reflects the local pressure gradient in the phases (see section 4.2.3) which relates to the counterflows there. Consequently, a more powerful thermocapillary effect must exist than in the $\operatorname{Ar} 1 \operatorname{Ar} 2$ nonequilibrium interfacial system. We shall check below in the systems to come if the effect truly depends on the spread between the limiting density distributions.

We see only slight quantitative differences between the temperature distributions in the systems (see Figure 42b). Nevertheless, these differences are very interesting from a qualitative point of view. Thus, the anomalous temperature $z$-dependence in the Ar1Ar2 system disappears in the Ar5Ar5 one. Instead, it exhibits constant temperatures and temperature $y$-gradients as function of $z$. Such a behaviour is exactly as intended when we set up the homophasic systems. The unphysical behaviour near the thermostated regions, e.g. the temperature jumps described in section 5.1.3, persists, however, or even slightly increases. As a result, somewhat lower temperatures establish at the centres, i.e. at $|y|=2.0 \mathrm{~nm}$, of the Ar5Ar5 system than at the same location in the Ar1Ar2 system. Other than that, the temperature $y$-gradients resemble their I-average in the heterophasic system (see Table 7). We shall readily trade in subsequent studies the difficulties connected to the anomalous temperature $z$-dependence against such an increased temperature drop, especially since its bias on the results remains rather limited.

Let us take another look at the densities. Figure 42a compares, for instance, specifically their $z$ profiles at $|y|=2.0 \mathrm{~nm}$ in the nonequilibrium interfacial systems with those in the corresponding equilibrium interfacial ones. The slightly greater differences in the case of the corresponding Ar5Ar5 systems result, mainly from the above-explained stronger disparity of the temperature jumps at the thermostated regions. These differences are, however, negligible, as in Ar1Ar2 systems from the previous chapters. A similarly close agreement exists also in many other observables or their standard deviations (see Figure 42) between the different Ar5Ar5 systems. Hence, we can confidently proceed our studies of the homophasic interfacial systems. In particular the results near the thermostated regions must, of course, still be taken with care.

We give the global observables in Table 14. The Ar5Ar5 nonequilibrium interfacial system exhibits a larger disparity between the temperature jumps at the thermostated regions, as established above. 
It manifests itself in a slightly smaller global temperature than in the Ar1Ar2 nonequilibrium interfacial system. As a result, the pressure and energy differences between the corresponding Ar5Ar5 systems increase somewhat compared to those between the corresponding Ar1Ar2 systems. We established already in the equilibrium interfacial systems (see section 5.3.1) the smaller system pressures and energies in the Ar5Ar5 than in the Ar1Ar2 mixture. Moreover, the Ar5Ar5 mixture exhibited a smaller thermal conductivity in the nonequilibrium one-phase systems (see section 5.3.2). Both trends occur similarly between the corresponding nonequilibrium interfacial systems. 


\subsection{Examination of the Parameters and Simplifications in the Homophasic Systems}

The Ar5Ar5 systems described in the previous chapter served additionally as a reference. We manipulated them in additional simulations so as to study the influence of further system parameters. Each of the sections below discusses the response to another manipulation. In particular the response in the intensity of the thermocapillary convection will be at the centre of our interest. Go back to section 4.2.3 for different definitions of this intensity. We shall omit, for the sake of brevity, other aspects of the system behaviour partly or even completely, that were also discussed in the previous chapters. Thus, the local standard deviations remain unmentioned. They vary in agreement with their previously discussed relations but, as a result, provide no new insights into the system behaviour. We carefully checked the omitted observables, nevertheless.

The first section 5.4.1 below addresses the finite size effects. In order to study them, we expanded the simulation system. Concurrently, we adjusted the particle numbers and thermostated temperatures so as to keep its overall density, average temperature and temperature gradient. The results to be discussed will show that the intensity of the convection converges against an upper limit when the system is expanded in one direction only, i.e. either $y$ or $z$. This limit depends apparently on two factors. On the one hand, there is the interfacial character of the phenomenon. Thus, the backflows, even if they are given more space (expansion in $z$-direction), do not spread arbitrarily deep into the phases. We are obviously dealing with a truly interfacial phenomenon. On the other hand, there is the interference between the forward and the backflows which is particularly strong at the centres of the roll cells. Its relevance decreases in comparison to the redirection of the flows at the thermostated regions as we expand the system along the temperature gradient, i.e. in $y$ direction. Both factors, so it will turn out later, keep, however, approximately their proportions if we expand the system simultaneously in both, i.e. $y$ - and $z$-, directions and maintain concurrently the difference between the thermostated temperatures instead of the temperature gradient.

We shall look in the subsequent sections at the system response to manipulations of other parameters. They have, different from the ones discussed before, a practical correspondence in an experiment. Think, for instance, of the temperatures established by the thermostats. We first reduced the temperature gradient. Section 5.4.2 discusses the details of these reductions and how 
the system responds to them. Its flows will turn out to be in direct proportionality with the slope of the temperature gradient. Moreover, the influences of the average system temperatures and pressures were studied. We discuss the associated system response in section 5.4.3. Generally speaking, the flows will be found to increase with decreasing pressure; they are the strongest if the temperatures remain somewhat below the line of the critical solution points. Finally, we varied the interactions of unlike particles through the Lorentz-Berthelot mixing parameter $\xi$ (see section 5.4.4), smaller values (i.e. a smaller miscibility) leading to stronger flows. A qualitatively similar trend exists in variations of the particle masses. Smaller ones, so the discussions below will show, favour the convection (see section 5.4.5). Note, we studied the influence of changing the relative mass ratio already in section 5.2.2.

Each of the above-mentioned parameter variations affects, of course, several hydrodynamic material properties at a time in a simulation. It is not easily possible to keep $n$ - 1 of them constant, as usually postulated in phenomenological thermodynamics. Hence, we skate on thin ice when we try to isolate the influence of individual parameters on the thermocapillary convection. Nevertheless, looking also at the variations of other observables in the sections below will give us some hints. Thus, there is a second property in addition to the intensity of the convection, namely the interfacial width, that responds as well strongly to the modifications of the mixing parameter, $\xi$ (see section 5.4.4). Both properties might thus even be interrelated. Similarly, the intensity and the viscosity seem to be interrelated (see e.g. section 5.4.5). These interrelations suggest an explanation for the influence of the system temperature and pressure on the thermocapillary convection described above. While the viscosity decreases in approaching the critical solution line, the interface widens. Its width increases only very little remote from the critical line. Both variations, however, seem not to be linear and may thus lead to a maximum in the intensity (see Figure 49b). Hence, we shall conclude that the convection intensifies with increasing temperature gradient and decreasing viscosity or interfacial width. Such a behaviour would also be in agreement with experimental results (see part 2). 


\subsubsection{System Dimensions}

Any simulation box, that can be thought of for molecular simulation, will always be small compared to macroscopic dimensions. The assumption of periodic boundary conditions, see section 3.2, alleviates this only partly. Hence, it is important to check to what extent the system size influences the computed observables. Its influence may be quite different for the different ones of them. Thus, the finite size error in the self-diffusion coefficient, for instance, decreases much slower with increasing system size than that in many other observables [spangberg2003]. See also the different publications by K. Binder et al. , e.g. [binder2000]. It is of particular importance to check our nonequilibrium interfacial systems also for such effects.

We increased, with respect to different reference systems, the dimensions perpendicular, $L_{y}$, and lateral, $L_{z}$, to the interfaces. In doing so, we concurrently adjusted the particle numbers so as to maintain the global system state. For the sake of simplicity, the system dimensions were simply doubled in each change by joining two identical configurations of a smaller system. Subsequently, a steadying simulation of 6 million time steps was conducted to obtain the new initial configuration for the production runs. When extending in $z$-direction, we conducted an additional equilibration of 2 million time steps prior to the steadying. In this equilibration, the system was thermostated to supercritical temperatures so that the steadying would result in two separate phases instead of four.

Let us begin by comparing the thermocapillary flows in the Ar5Ar5 reference system, N-Ar5Ar5-

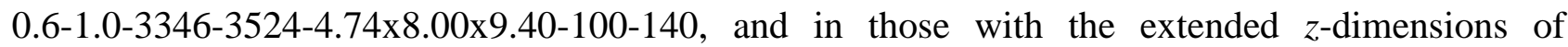
$L_{z}=18.8 \mathrm{~nm}, \mathrm{~N}-\operatorname{Ar} 5 \operatorname{Ar} 5-0.6-1.0-6692-7048-4.74 \times 8.00 \times 18.8-100-140$, and of $L_{z}=37.6 \mathrm{~nm}, \mathrm{~N}-\operatorname{Ar} 5 \operatorname{Ar} 5-$ 0.6-1.0-13384-14096-4.74x8.00x37.6-100-140. Figure 44a compares the vector plots of these flows. Moreover, we extracted characteristic profiles of the com velocity components. Thus, Figure $44 \mathrm{~b}$ shows the $y$-velocities at the centres between the thermostated regions, $|y|=2.0 \mathrm{~nm}$, and the $z$-velocities at the thermostated regions, $|y|=0.4 \mathrm{~nm}$ and $|y|=3.6 \mathrm{~nm}$, as function of $z$. Apparently, the roll cells remain associated with the interfaces, i.e. their centres do not recede from the interfaces to the same degree that the system size increases. Thus, the $y$-velocity at the centres between the interfaces is clearly reduced in the wider system $\left(L_{z}=18.8 \mathrm{~nm}\right)$ and even becomes zero in the widest one $\left(L_{z}=37.6 \mathrm{~nm}\right)$. Such a behaviour confirms again that the found convection is indeed an interfacial and not a bulk phenomenon. 
Let us also compare the thermocapillary convection in systems with different $y$-dimensions,

N-Ar5Ar5-0.6-1.0-6692-7048-4.74x8.00x18.8-115-125

N-Ar5Ar5-0.6-1.0-13384-14096-4.74x16.0x18.8-110-130

and N-Ar5Ar5-0.6-1.0-26768-28192-4.74x32.0x18.8-100-140.

It makes sense in such a comparison to have the same temperature $y$-gradient in these systems. (Note that we shall study its separate influence thoroughly later in section 5.4.2.) The one with the temperature difference of $\Delta T=T_{H^{-}} T_{C}=10 \mathrm{~K}, \mathrm{~N}-\mathrm{Ar} 5 \operatorname{Ar} 5-0.6-1.0-6692-7048-4.74 \times 8.00 \times 18.8-115-125$, was used as the basis of comparison, here. As a result, we can extend the $y$-dimension significantly without having to increase the temperature difference above its level in previous simulations. Note also that the $z$-dimension has twice its standard length. We thus prevent a rearranging of the interfaces in other directions than perpendicular to the $y$-axis. The ratio of lateral extension to the width of the phases would otherwise become too unfavourable.

Figure 45a compares the thermocapillary convection for different $y$-dimensions. It clearly increases with increasing length $L_{y}$. Plotting the intensities of the convection as a function of the $y$-dimension suggests that they converge toward a limit (see Figure 45b). Such a convergence hints at the role of the fluid viscosity. The turnaround flows at the thermostated regions loose importance in systems with larger $y$-dimensions. Nevertheless, the convection does not increase infinitely (which it could since we do not limit the amount of energy that the thermostats feed into and out of the system). Thus, there must be a second mechanism, friction, that slows down the thermocapillary effect. We shall look at this mechanism in more detail in section 5.5.2.

In the expansions of the $z$-dimension above, we saw that a convection roll cell does not spread without limits into the phases. It rather maintains approximately the same dimension in $z$ - and as in $y$-direction. See, for instance, Figure 44. We compare here two examples of systems with such a flow pattern. The second one of them, N-Ar5Ar5-0.6-1.0-26768-28192-4.74x16.0x37.6-100-140, has, in comparison to the first one, N-Ar5Ar5-0.6-1.0-6692-7048-4.74x8.00x18.8-100-140, been doubled (in the way explained above) in both the $y$ - and the $z$-direction. The systems are, however, subject to the same temperature difference of $\Delta T=T_{H^{-}} T_{C}=40 \mathrm{~K}$, now. We can thus try, with all due care, to extrapolate to a situation where the system dimensions and the temperature gradients have magnitudes more typical of "physical experiments". Figure 46 shows the convection in both systems. The maximum velocity components (see Table 21) differ by a factor of less than 1.3. It 
might be even somewhat closer to unity, if we had compared larger systems. They feature an even lower slowdown of the flows by the redirection at the thermostated regions, as discussed above for the exclusive doubling of the $y$-dimension. It thus seems reasonable to assume that the velocities in our simulations have roughly the same magnitudes as in typical experiments, see section 2.1.1. 


\subsubsection{Temperature Difference}

Using the default Ar5Ar5 system, N-Ar5Ar5-0.6-1.0-3346-3524-4.74x8.00x9.40-100-140, as a reference, we varied in additional simulations both thermostated temperatures so as to study specifically the influence of their difference on the thermocapillary convection. To be more specific, the difference is reduced from the default value of $\Delta T=T_{H^{-}} T_{C}=40 \mathrm{~K}$ to values of $\Delta T=20 \mathrm{~K}$ in N-Ar5Ar5-0.6-1.0-3346-3524-4.74x8.00x9.40-110-130 and of $\Delta T=10 \mathrm{~K}$ in N-Ar5Ar5-0.6-1.0-33463524-4.74x8.00x9.40-115-125. Figure 47a compares the com velocities obtained in the different simulations. Moreover, we show in Figure $47 \mathrm{~b}$ the intensity of the thermocapillary convection. This intensity can be characterised, as explained previously in section 4.2.3, by using the maximum com $y$-velocities or the spatial velocity correlation function $C$. The so-measured intensity decreases linearly with decreasing temperature difference, $\Delta T$. This confirms once again that our systems lie within the scope of the linear response theory [kubo1957a], [kubo1957b]. 


\subsubsection{System Pressure and Temperature}

The previous section addressed additional simulations of the Ar5Ar5 reference system. They have different temperature differences between the thermostated regions but the same the global system state, i.e. the global pressure and temperature. We now look at simulations in which we maintained the temperature difference but varied the global pressure and temperature. The pressure can only be adjusted indirectly through other system constraints, since our simulations are run, for reasons explained in chapter 4.1, under $\left(N V T_{1} T_{2}\right)$-conditions. To do so, we used the Ar5Ar5 reference system as a starting point. At first, the thermostated temperatures were set such that the intended global temperature established in a steadying simulation of 6 million time steps. Afterwards, if necessary, we gradually adjusted the system $x$-dimension, $L_{x}$, in further steadying simulations, each of 4 million time steps, until the desired pressure was reached. The thus obtained terminal system configuration served as a starting point for the actual production run. Since our variations are only to such a degree that no phase transitions occur, the pressure is an injective function of the $x$ dimension, $L_{x}$, and the thermostated temperatures, $T_{C}$ and $T_{H}$. We varied specifically the $x$ dimension in order not to affect the proportions of the thermocapillary convection vortices. Remember from section 4.2 that the system is homogeneous along this dimension.

Figure 49a compares the thermocapillary convection in a first set of similar systems:

N-Ar5Ar5-0.6-1.0-3346-3524-4.74x8.00x9.40-80-120,

N-Ar5Ar5-0.6-1.0-3346-3524-4.74x8.00x9.40-100-140,

N-Ar5Ar5-0.6-1.0-3346-3524-4.74x8.00x9.40-120-160.

Their constraints differ only in terms of the average temperature $T_{C H}=0.5\left(T_{C}+T_{H}\right)$. The higher it is, the higher is the global pressure (see Table 24). We indicate these pressures and temperatures again in Figure 48 in a cut of the Ar5Ar5 PxT-diagram at the approximate system composition of $x_{A r(5)} \approx 0.5$. Figure $49 \mathrm{~b}$ displays the intensities of the convection as a function of the global pressure or temperature. We defined these intensities previously in section 4.2.3. The convection clearly strengthens with decreasing global temperature and pressure.

In order to find out more about the isolated pressure dependence of the convection, we simulated another set of systems: 
N-Ar5Ar5-0.6-1.0-3346-3524-4.50x8.00x9.40-100-140,

N-Ar5Ar5-0.6-1.0-3346-3524-4.74x8.00x9.40-100-140,

N-Ar5Ar5-0.6-1.0-3346-3524-5.00x8.00x9.40-100-140.

They differ only in the $x$-dimensions and thus in the global pressures (see Figure 48). The convection intensifies with decreasing pressure (see Figure 49). Note that this pressure dependence is stronger than in the set of systems above that have a different pressure and temperature range. Such a behaviour suggests that, given constant pressures, higher temperatures favour a stronger convection.

We checked the above-suggested influence of the temperature in another set of systems:

N-Ar5Ar5-0.6-1.0-3346-3524-4.39x8.00x9.40-80-120,

N-Ar5Ar5-0.6-1.0-3346-3524-4.74x8.00x9.40-100-140,

N-Ar5Ar5-0.6-1.0-3346-3524-5.22x8.00x9.40-120-160.

Their $x$-dimensions were adjusted in the way described above so that all systems have the same global pressure. These system states are also indicated in the PxT-diagram in Figure 48. Different from our expectations, the thermocapillary convection weakens, however, for both the higher and the lower temperatures (see Figure 49). Consequently, a certain intermediate temperature must lead to the strongest flows. We can tell from the changes that it lies close to the critical end point temperature.

To find out more about the dependence of this maximum, additional systems were simulated. One of them, N-Ar5Ar5-0.6-1.0-3346-3524-4.44x8.00x9.40-100-140, has the same pressure,

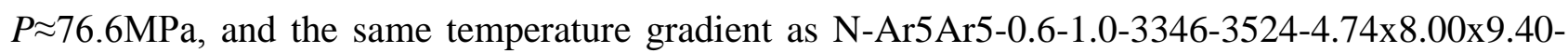
120-160, but at a lower temperature level. It comes with a slightly stronger convection. Another system, N-Ar5Ar5-0.6-1.0-3346-3524-5.46x8.00x9.40-100-140, has the same pressure, $P \approx 13.1 \mathrm{MPa}$, and the same temperature gradient as N-Ar5Ar5-0.6-1.0-3346-3524-4.74x8.00x9.4080-120, but at a higher temperature level. It comes with a slightly weaker convection. Hence, we can assume the above-mentioned maximum of the convection intensity decrease with decreasing pressures. Since lower pressures additionally favour the convection, as seen above, we can assume it to intensify even more in our systems if their global state descends further along the liquid-liquidvapour three phase line. 
Note that the pressure and temperature dependence of the convection is, at least at lower temperatures, opposite to the one of the viscosity of liquid Argon [younglove1986] and, presumably, also of our low concentration mixtures. Both become more viscous at higher pressures and lower temperatures. In approaching the critical solution temperatures, the lower viscosities presumably favour the convection too little to compensate for the decreasing spatial variation at the interfaces. 


\subsubsection{LB Mixing Parameter}

We manipulated in additional simulations of the A5Ar5 reference system the parameters that relate directly to the substances. Chapter $\mathbf{5 . 3}$ addressed already the effect of such manipulations in the comparisons of the Ar1A2 with the Ar5Ar5 reference systems. It was the main purpose of these manipulations, however, to establish the better approximation of reality for symmetric interactions between like particles. Here, we focus on the influence, that the LJ-parameter values have, based on this symmetry. Thus, the Lorentz-Berthelot mixing parameter $\xi$ was varied within reasonable limits. It specifies, as explained in section 4.1.2, the interatomic forces and potentials between unlike particles based on those of like particles.

In addition to the Ar5Ar5 reference system, N-Ar5Ar5-0.6-1.0-3346-3524-4.74x8.00x9.40-100140 , similar systems with the mixing parameter values of $\xi=0.5$, N-Ar5Ar5-0.5-1.0-3346-3524$4.74 \times 8.00 \times 9.40-100-140$, and of $\xi=0.7$, N-Ar5Ar5-0.7-1.0-3346-3524-4.74x8.00x9.40-100-140, were simulated. Figure 50a compares the thermocapillary convection for the different parameter values. Smaller values, i.e. essentially higher miscibilities, lead to weaker flows. We show in Figure $50 \mathrm{~b}$ their intensities as defined previously in section 4.2.3. Their response correlates with that to the variation of the global pressures and temperatures near the line of the critical solution points (see section 5.4.3). The closer the system state lies to this line, the weaker is the occurring thermocapillary convection. A similar behaviour applies in the present systems except that the line of critical solution points now mainly shifts in comparison to the system pressures and temperatures, when we manipulate the mixing parameter (see Figure 51). We estimated the solution points in the way described in part $\mathbf{1 1}$ by using the virial equation of state. Although they are only rough estimates, they exhibit the correct qualitative behaviour. The larger the distance is between the state of a given system and its applicable critical line, the stronger is the thermocapillary convection. 


\subsubsection{Particle Masses}

Remember from section 5.2.2 that the static observables in an equilibrium system should remain unaffected by manipulations of the particle masses. Changes that occur nevertheless in such a simulation could rather be considered as a means to assess its deviation from reality. We found hardly any differences between the static observables in the heterophasic interfacial systems with the different masses of the ArB particles. The dynamic observables, on the other hand, depend on the particle mass. We could see their dependence clearly in the differences between the corresponding nonequilibrium systems.

Our thermostats have difficulties in handling spatially variable conductivities along the thermostated regions (see section 5.2.2). Hence, we manipulated additionally the masses, generally called $m_{A r A}$ and $m_{A r B}$, of both particles simultaneously in the homophasic nonequilibrium interfacial and its corresponding systems. As explained in section 4.1.2, the so-changed particles have new names, namely Ar6 with a mass of $m_{A r 6}=3.995 \mathrm{amu}$ in the system, N-Ar6Ar6-0.6-1.0-3346-35244.74x8.00x9.40-100-140, and Ar7 with a mass of $m_{A r 7}=399.5 \mathrm{amu}$ in the system, N-Ar7Ar7-0.6-1.03346-3524-4.74x8.00x9.40-100-140. Both exhibit a qualitatively similar thermocapillary convection as the Ar5Ar5 reference system, N-Ar5Ar5-0.6-1.0-3346-3524-4.74x8.00x9.40-100-140 (see Figure 52a). The quantitative differences turn out, however, more interesting than one might expect at first sight. They help us distinguish the static from the dynamic aspects in the system behaviour without having to think of the different properties of the phases.

Let us take a look at selected derivational properties in the systems. While the intensity (defined in section 4.2.3) of the convection (see Figure 52b) and the thermal conductivity decrease (see Table 21) with increasing mass, the viscosity increases. We can estimate it from the magnitude of the local com velocity standard deviations, as explained in section 5.2.2. Other properties, that relate to the thermocapillary convection in the previous studies, differ only within the numerical accuracy of the results. In particular the Soret coefficients in the phases hardly exhibit any differences (see Table 21). Thus, all three systems feature a similar balance between the conflicting tendencies discussed in our thought experiment (see section 4.2.3), namely to separate in $y$-direction and to establish local equilibria across the interfaces. Moreover, the $y$-gradient of the normal stresses perpendicular to the interfaces, $S_{z z}$, has approximately the same value in the three systems (see 
Table 22). It is a measure for the strength of the driving force behind the convection, as explained in sections 5.1.3. In other words, there is the possibility that the thermocapillary convection, although it has the same driving force, occurs with different intensities in a system, depending on the viscosity. Verification of this result as a general rule in other systems would allow disregarding the viscosity in the research into the thermocapillary effect. Finally, the global pressures and temperatures hardly respond to the changes of the particle masses (see Figure 53). 


\subsection{Advanced Studies of the Homophasic System Behaviour}

Here, we return for a more detailed analysis to some of the Ar5Ar5 systems described above. First, we shall look at the local particle densities and their spatial distribution (see section 5.5.1). It will turn out to be, as postulated in our thought experiment from section 4.2.3, in between the distribution to expect from the corresponding equilibrium interfacial systems and the one to expect from the corresponding nonequilibrium one-phase systems. The densities at the centres between the thermostated regions are close to the ones in the corresponding equilibrium interfacial system. Away from the interfaces, the densities approximate the ones in the corresponding nonequilibrium one-phase system. Different particle specific motions accompany this density distribution.

We will then show by looking at the distribution of selected stress tensor elements that our systems behave in agreement with hydrodynamics (see section 5.5.2). Thus, a pressure gradient, i.e. a gradient of the normal stresses, drives the back flows away from the interfaces, while friction, i.e. a gradient of the shear stresses, keeps the convection from increasing infinitely. In the interfacial regions, the situation is, however, less clear. Gradients of the normal stresses or of the shear stresses might act as driving force or as a limiting factor of the convection. 


\subsubsection{Analysis of the Spatial Density Distribution}

Let us first review what is known about the density distributions in a nonequilibrium interfacial system in more detail. As we saw, for instance in the Ar5Ar5 reference system from chapter 5.3, the densities vary sigmoidally in $z$-direction, i.e. in going from one phase to the other (see Figure 54a), and linearly along the $y$-direction, i.e. along the temperature gradient (see Figure 54b). These variations reflect the competing tendencies that our thought experiment from section 4.2.3 postulates in the system. We estimated the density distributions to expect if either the first or the second tendency dominates. The estimation of both limiting cases will be described in detail below. Moreover, we shall compare them to the original density distribution, that we computed in simulations of selected Ar5Ar5 nonequilibrium interfacial systems, in order to confirm this postulate.

Remember from the studies in the previous chapters that the dominant transport mechanisms are the molecular ones in our systems and not convection. Hence, we can assume it to have little effect on the local thermodynamic states. Instead, three intensive variables specify according to Gibbs's phase rule [gibbs1875], [findlay1951] unambiguously a local state in a phase, e.g. both partial densities and the temperatures. Since the different density distributions to compare below involve approximately the same pressures and temperatures, we can simply tell the identity of the local states from that of the local densities. For the sake of simplicity, only these densities shall thus also be considered in detail in the subsequent studies.

When one approaches the interface from the bulk, the tendency of the nonequilibrium interfacial systems to establish local equilibria between both neighbouring phases becomes more and more apparent. It manifests itself in the sigmoidal density z-profiles which resemble those in an equilibrium interfacial system. This similarity is quantitative at the centres between the thermostated regions (see Figure 54c). We additionally computed a z-profile of the spatially averaged density $y$-gradients in the investigation regions (see Figure 54d). Linear regressions of the density data at every $z$-location - section 4.3.5 describes them in more detail - produce these gradients. They provide, if only approximately, another impression of the interfacial heterogeneity. Note their $z$-shaped interfacial variation, i.e. they vary between limiting values of different signs in both phases and overshoot in between them. 
Thermal diffusion (Ludwig-Soret effect [ludwig1856], [soret1879], [soret1881], [eilert1914]) dominates away from the interfaces. We can tell so from the convergence of the local system behaviour against the one in the corresponding nonequilibrium one-phase system. Take for instance the partial density $y$-gradients. They tend to approach in the phases of the nonequilibrium interfacial system N-Ar5Ar5-0.6-1.0-3346-3524-4.74x8.00x9.40-100-140 the limiting values of $\partial \rho_{M a j} / \partial y=-0.71 \mathrm{~nm}^{-4}$, and $\partial \rho_{M i n} / \partial y=0.06 \mathrm{~nm}^{-4}$, found in its corresponding nonequilibrium one-phase system,

N-Ar5Ar5-0.6-1.0-6744-223-4.74x8.00x9.40-100-140,

remote from the interfaces (see Figure 54d). The locally computed formal Soret coefficients show a similar convergence behaviour. Figure $54 \mathrm{f}$ shows them as function of as the distance, $d$, from the Gibbs dividing surfaces. The convergence becomes even more clear in the longer systems

N-Ar5Ar5-0.6-1.0-6692-7048-4.74x8.00x18.8-100-140, and N-Ar5Ar5-0.6-1.0-13384-14096-4.74x8.00x37.6-100-140,

that have the same corresponding nonequilibrium one-phase system as N-Ar5Ar5-0.6-1.0-33463524-4.74x8.00x9.40-100-140. Its partial density y-gradients and Soret coefficients are assumed in the phases of the longest system. Thus, the spatial average of the local coefficient in the regions outside the roll cells produces a value of $S_{T}=-0.013 \mathrm{~K}^{-1}$. It differs only little from the one in the nonequilibrium one-phase system $S_{T}=-0.014 \mathrm{~K}^{-1}$. The minor difference between both values might be due to the slight oversaturation in the nonequilibrium one-phase system (see further below) and the somewhat lower pressures in the nonequilibrium interfacial system (see Table 24).

\section{Comparison to the Constructed Local Equilibrium Density Distribution}

As a check, we constructed the density distribution to expect in the nonequilibrium interfacial system, N-Ar5Ar5-0.6-1.0-3346-3524-4.74x8.00x9.40-100-140, according to the considerations above. The density $z$-profiles were computed in several simulations of equilibrium interfacial systems,

E-Ar5Ar5-0.6-1.0-3346-3524-4.74x8.00x8.71-100,

E-Ar5Ar5-0.6-1.0-3346-3524-4.74x8.00x9.04-110,

E-Ar5Ar5-0.6-1.0-3346-3524-4.74x8.00x8.71-120, 
E-Ar5Ar5-0.6-1.0-3346-3524-4.74x8.00x9.83-130,

and E-Ar5Ar5-0.6-1.0-3346-3524-4.74x8.00x10.4-140,

at different temperatures and pressures, comparable to the ones in the non-equilibrium system. We estimated the $y$-locations at which these temperatures are found in the nonequilibrium systems and assumed the density $z$-profiles of the equilibrium interfacial systems at these locations (see Figure 55). In doing so, the profiles had to be realigned separately for each interface by shifting them in $z^{-}$ direction until the $z$-locations of the Gibbs dividing surfaces coincide. We then resampled the thus obtained density distribution by linear interpolation of each shifted z-profile and recomputed the density data at common $z$-coordinates. Figure 56a shows such a constructed distribution for the Ar5Ar5 reference system as an example.

Let us have a look at the density z-profile at the centres between the thermostated regions, i.e. $|y|=2.0 \mathrm{~nm}$ here. It strongly resembles, as already stated, the one in the corresponding equilibrium interfacial system of the same temperature. The constructed density distribution (see Figure 56b) also agrees very well, which shows that our linear interpolation scheme does not introduce any bias. When one approaches the thermostated regions, both the original and the constructed density distribution start to differ. We can see their differences particularly well by comparing the density $y$ profiles in the phases (see Figure 56c). The partial density profiles are clearly steeper in the constructed distribution. We additionally computed $y$-gradients from the constructed profiles by linear regression, as described in section 4.3.5. They exhibit steeper density $y$-gradients also in the interfacial regions (see Figure 56d). Hence, our thought experiment from section 4.2.3 is confirmed in that the local phase equilibria can in general not establish along the interface.

The distribution of the temperatures was also discussed before in chapter 5.4. They vary linearly between the thermostated regions. An additional $z$-dependence cannot be established anymore in the homophasic systems. Nevertheless, the temperature jumps continue to exist at the thermostated regions. These jumps turned out, however, in chapter $\mathbf{5 . 1}$ to be often too small to disturb the system behaviour significantly. The same holds also for the systems considered in the present chapter. Hence, we do not go further in into the temperature jumps here. Similarly, the velocity distribution shall only be described, if necessary to make a point. 


\section{Comparison to the Density Distribution in the Nonequilibrium One-Phase System}

We also simulated the corresponding nonequilibrium one-phase system, N-Ar5Ar5-0.6-1.0-6744223-4.74x8.00x9.40-100-140. It reproduces the nonequilibrium behaviour of the phases separated from each other, as explained in chapter 4.1. We can use the thus obtained density distribution as another standard, in addition to the com velocities, to judge how far the influence from the interfaces spreads into the phases. Figure 56d compares the density $y$-profiles in the corresponding nonequilibrium one-phase system to those in the phases of the nonequilibrium interfacial reference system, N-Ar5Ar5-0.6-1.0-3346-3524-4.74x8.00x9.40-100-140. Its somewhat steeper profiles indicate that the phases, even as far away as (computationally) possible from the Gibbs dividing surfaces (see section 4.3.5), do not yet exhibit full bulk properties. Real bulk properties exist only outside the convection roll cells, as seen in the expanded system, N-Ar5Ar5-0.6-1.0-13384-140964.74x8.00x37.6-100-140 from section 5.4.1. The depth of the influence of an interface into the phases can thus be estimated from the density $y$-gradients to be about $5.0 \mathrm{~nm}$ (or roughly 15 particle diameters) under these thermodynamic conditions. This distance is more than twice the one of 2.0nm (or roughly 6 particle diameters) beyond which the densities are (within our uncertainties) constant functions of $z$ (see Figure 60). Apparently, there is a coupling between the convection in the nonequilibrium interfacial system and the deviation of its density distribution from that in the corresponding nonequilibrium one-phase system. We shall study this coupling in more detail further below using the particle specific streaming velocities.

Let us, however, first look at how the segregation of the substances due to the thermal diffusion varies in an interfacial region. Since its local states cannot exist in a separate "bulk" phase, it is difficult to determine the exact strength of this segregation. We estimated it at least by extrapolating its concentration dependence from outside the miscibility gap (see Figure 57). To do so, a series of nonequilibrium one-phase systems was simulated:

N-Ar5Ar5-0.6-1.0-6967-0-4.71x8.00x9.40-100-140,

N-Ar5Ar5-0.6-1.0-6917-50-4.71x8.00x9.40-100-140,

N-Ar5Ar5-0.6-1.0-6867-100-4.72x8.00x9.40-100-140,

N-Ar5Ar5-0.6-1.0-6817-150-4.73x8.00x9.40-100-140,

and N-Ar5Ar5-0.6-1.0-6767-200-4.74x8.00x9.40-100-140. 
All of them have the same temperature constraints as well as $y$ - and $z$-dimensions as the system, $\mathrm{N}$ Ar5Ar5-0.6-1.0-6744-223-4.74x8.00x9.40-100-140. The relative concentrations were however varied. We adjusted the $x$-dimension in the steadying of each system until its pressure equalled the one of the nonequilibrium interfacial system. Then followed an additional steadying simulation of another 6 million time steps. The thus obtained configurations finally served as starting points for production runs of 15 million time steps.

The density $y$-gradients in any one of the above-mentioned systems are always steeper than in the other ones with a smaller mole fraction, $x_{A r}(5)$, of the majority component. Figure 58 plots the slope of these gradients as function of the composition of the different systems. We see a non-linear dependence. It is clearly increased in the oversaturated nonequilibrium one-phase systems. Figure 58 additionally shows for comparison the binodal curve that we determined in several simulations of equilibrium interfacial systems. According to it, a segregation of the mixture into separate phases occurs for compositions between $x_{A r(5)}=0.02$ and $x_{A r(5)}=0.98$, given the pressures and temperatures in the one-phase systems. We neglect here the additional error in them due to the thermostating, e.g. the temperate jumps of different magnitudes at the thermostated regions and the thus slightly reduced average system temperatures. The Soret coefficient, indicated as well in Figure 58, shows, by tendency, a similar concentration dependence as the slope of the density $y$-gradients. These data may be subject to larger uncertainties since their computation involves several observables. Nevertheless, we can affirm that the Soret coefficient remains at first on a level of approximately $S_{T}=-0.55 \mathrm{~K}^{-1}$ with decreasing mole fraction and then clearly decreases as it passes below the binodal curve.

The concentration dependence of the $y$-gradients and of the Soret coefficient in the non-equilibrium one-phase systems is in keeping with the observations made in the nonequilibrium interfacial systems. Their interfacial compositions lie as well inside the "equilibrium" miscibility gap of the mixture. We would thus expect steeper density $y$-gradients in the interfacial regions than in the phases. This is what is found, see Figure $54 \mathrm{~d}$. We have described the $z$-shaped interfacial variation of the density $y$-gradients already above in more detail. Hence, its occurrence makes not only sense in the light of the density distribution to expect from the related equilibrium interfacial systems but also in the light of the concentration dependence of the thermal diffusion. 


\section{Relative Motion of Both Substances}

Let us first note for the sake of clarity that "diffusion" may have different meanings in irreversible thermodynamics and statistical mechanics. We take the term here as a spatially and temporally averaged particle velocity with respect to the inertial system. Regular and thermal diffusion cancel each other in a stationary nonequilibrium one-phase system. The traditional Ludwig-Soret effect [ludwig1856], [soret1879], [soret1881], [eilert1914] conforms to this cancellation. It means, more precisely, that the net fluxes of the different substances are zero. We verified its applicability again in our one-phase systems by determining the locally averaged particle com velocities for each kind (see section 4.3.2). We shall denote them hereafter as particle specific, for the sake of simplicity. Figure 59a shows these velocities as an example in the system, N-Ar5Ar5-0.6-1.0-6744-2234.74x8.00x9.40-100-140. The absence of any identifiable convection patterns for either kind of particle is confirmed.

The nonequilibrium interfacial systems exhibit, however, different particle specific com velocities. In fact, they always have similar distributions. For the sake of brevity, we thus discuss only one set of them here, using as an example the system N-Ar5Ar5-0.6-1.0-13384-14096-4.74x8.00x37.6-100140. Figure 59b shows a plot of the local velocities as functions of $y$ and $z$. Since the exact numerical results are difficult to determine in such vector plots, the Figure 59b shows moreover the different velocity components as function of $z$ only. When assessing the relative motion of the particles in $y$-direction, we focus specifically on the centres between the thermostated regions, i.e. $|y|=2.0 \mathrm{~nm}$ in the present example (see Figure 60). Some of the highest $y$-velocities occur there, so that any particle specific difference can be clearly identified. In addition, Figure 60 shows once again the overall com velocities and the three regions used in the discussion below. These regions are defined with respect to the locations of the roll cells. Within each region we spatially averaged the overall $y$-velocities and those of the majority and minority components (see Table 34). The thus obtained averages give a clearer picture of the relative motion in the system. Finally, we also considered the local densities and density $y$-gradients, shown as well in Figure 60. They are known to control the relative motion of the particles in agreement with Fick's first law of (regular) diffusion [fick1855]. We have shown above in the nonequilibrium one-phase systems how its constant of proportionality (the diffusion coefficient) depends on their composition. 
In the region outside the roll cells both particle specific velocities are, as expected at the beginning of this section, approximately zero (see Table 34): the partial densities and their $y$-gradients resemble those in the corresponding nonequilibrium one-phase system. In approaching the interfacial regions, the partial density $y$-gradients steepen. This typically entails stronger diffusion according to Fick's law. The local composition, on the other hand, hardly varies across the regions of the backward flows. Accordingly, regular diffusion dominates there, so that, in keeping with the $y$-gradients, the majority component flows faster than the minority one (see as well in Table 34). Both flows must have opposite directions because of the different signs of the gradients.

Closer to the Gibbs dividing surface, i.e. in the region of the forward flows, we cannot tell a priori from the densities and their gradients which type (regular or thermal) of diffusion dominates. Regular diffusion should weaken in agreement with the flattening $y$-gradients, but we do not know about the thermal diffusion. As mentioned before, the local composition in the interfacial region can hardly be reproduced in nonequilibrium one-phase systems. Nevertheless, regular diffusion seems to remain dominant since both particle specific velocities differ in agreement with the partial density $y$-gradients, i.e. the majority component flows slower than the minority component (see as well in Table 34). If regular diffusion remains dominant, the thermal one must be weaker as well. We can tell so from the Soret coefficient, which is also defined as the thermal diffusion coefficient divided by the molecular diffusion coefficient, approaching zero right at the Gibbs dividing surface (see e.g. Figure 54f) where equimolar local compositions occur. It is problematic from the irreversible point of view if some of the phenomenological coefficients - maybe, due to their coupling, also the molecular diffusion or the heat conduction coefficient - decrease because the determinant of the phenomenological coefficients may eventually assume undefined values. A solution might be, to postulate a new transport mechanism that comes into action in the interfacial region, instead. This mechanism is then apparently a convective one, as it has been for instance predicted by Hampe [1980]. 


\subsubsection{Analysis of the Local Stresses}

As explained in section 2.2, a flow is said, from a hydrodynamic point of view, to be driven by "local stresses". Each of these stresses is thought to act, as force per unit area, on one of three mutually perpendicular cut surfaces of a small volume of liquid. In a fluid phase, one typically associates the normal stresses (diagonal elements of the stress tensor) with local pressures and the shear stresses (off-diagonal elements) with friction (see chapter 2.2). Frictional forces and those from the spatial variation of the pressure must, according to the Navier-Stokes equation [spurk1996], [landau2003], cancel each other in a free flow at low Reynolds numbers. In our interfacial systems, from a microscopic point of view, the situation is less clear. We shall nevertheless discuss below in how far these macroscopic concepts can be transferred to our present MD simulation approach. The flows in our interfacial systems have as well only small Reynolds numbers, as mentioned for instance in sections 5.1.3 or 5.3.3. Hence, no local forces should apply on a fluid element. We cannot, however, interpret the components of these forces as sketched above. Thus, any local pressure is, if at all, only ambiguously defined due to the anisotropy of the normal stresses at an interface. Moreover, shear stresses could possibly drive the thermocapillary convection by a mechanism similar to the one in thermal creep (see section 2.3.1). For a better understanding of the thermocapillary effect in these terms, we determined the local stresses in our homophasic nonequilibrium interfacial systems.

A suitable spatial resolution had to be found first. We chose it by trial and error as described in section 4.3.4. Since all our non-equilibrium interfacial systems feature similar stress distributions, we shall discuss them here only once, using the system N-Ar5Ar5-0.6-1.0-3346-35244.74x8.00x9.40-100-140 as an example. Figure 61 shows the different normal stresses as function of $y$ and $z$. They resemble, at first sight, the one in an equilibrium interfacial system. Thus, in going from one phase to the other, the normal stresses perpendicular to the interfaces remain constant while the ones parallel to them exhibit a minimum. All normal stresses assume identical values sufficiently away from the interfaces.

In the nonequilibrium interfacial systems, however, we see an additional $y$-dependence of the normal stresses. It is particularly strong in the interfacial regions. There, the lateral normal stresses, $S_{y y}$, decrease when moving toward the hot region. Further away from the interfaces, in the regions 
of the backward flows, the same stresses slightly increase. We plot, for instance, the $y$-gradient of the normal stresses, $S_{y y}$, again as function of $z$ in Figure 62. Note in particular that this gradient represents also a local $y$-force. To understand why, we must remember section $\mathbf{2 . 2}$ where we rewrote the local volume force as divergence of the stress tensor:

$\mathbf{f}=\left(\begin{array}{l}f_{x} \\ f_{y} \\ f_{z}\end{array}\right)=\nabla \cdot \mathbf{S}=\left(\begin{array}{l}\partial S_{x x} / \partial x+\partial S_{y x} / \partial y+\partial S_{z x} / \partial z \\ \partial S_{x y} / \partial x+\partial S_{y y} / \partial y+\partial S_{z y} / \partial z \\ \partial S_{x z} / \partial x+\partial S_{y z} / \partial y+\partial S_{z z} / \partial z\end{array}\right)$.

Visualise from this definition the spatial dependence of the above-mentioned $y$-forces. They always point in the same direction as the local flows, i.e. from hot to cold in the interfacial regions and the other way around further away from them.

In addition to the $y$-gradient of the lateral normal stresses, $S_{y y}$, the $z$-gradient of the shear stresses, $S_{z y}$, represents a local $y$-force. These shear stresses decrease with $\mathrm{z}$ in the regions of the backward flows and increase in the interfacial regions (see Figure 61c). Such a variation represents, according to equation (5-2), $y$-forces that always act so as to retard the roll cells. We show also the interatomic and kinetic contributions to the shear stresses in Figure 61c. Apparently, the individual particle motion hardly contributes to the retardation of the roll cells.

There must be, due to the small Reynolds numbers in our systems, a balance between the $y$-forces from the normal stresses, $S_{y y}$, and those from the shear stresses, $S_{z y}$. We checked this by looking at selected spatial averages of the $y$-gradients. At first, all $y$-forces from the normal stresses were spatially averaged. In doing so, we distinguished several regions according to the sign of these $y$ forces. Figure 62 compares the thus obtained averages in the different regions with the actual data. Then, we estimated the $z$-gradients by linear regression of the spatially averaged shear stresses in the same regions. As expected, the $y$-gradients of the normal stresses have approximately the same absolute values as the $z$-gradients of the shear stresses, but an opposite sign (see Figure 62). An even better agreement can be achieved by bringing the widths and the spatial resolution of the differently oriented cut surfaces to a better match. As a result, we obtain e.g. values of $\partial S_{y y} / \partial y=-0.55 \mathrm{pN} / \mathrm{nm}^{3}$ vs. $\partial S_{y z} / \partial z=0.62 \mathrm{pN} / \mathrm{nm}^{3}$ in the one and of $\partial S_{y y} / \partial y=-0.52 \mathrm{pN} / \mathrm{nm}^{3}$ vs. $\partial S_{y z} / \partial z=0.59 \mathrm{pN} / \mathrm{nm}^{3}$ in the other interfacial region. The differences lie well within the numerical accuracy of our methods. 
We take the applicability of the Navier-Stokes equation as a confirmation of the approximation of reality by our systems. The interpretation of the microscopic system behaviour in terms of this macroscopic model provides, however, no new insights into the thermocapillary effect itself. Let us muse some more about the reasons before we go on with further results in the next chapter. Although the contribution from the normal stresses, $S_{y y}$, always points in the $y$-direction of the flows, it would be premature to consider them as the "driving force" behind the thermocapillary convection. It may just as well be the consequence of the shear stresses, $S_{y z}$, that come as well into existence when we establish a temperature gradient in an initially equilibrium interfacial system. Cause and effect are hard to distinguish in our stationary NEMD simulations. Nonstationary ones would be more appropriate but are still subject to research. We therefore omit them here. Nevertheless, we would like to add that the perpendicular normal stresses, $S_{z z}$, might provide a hint to the puzzle.

In the phases, the lateral normal stresses, $S_{x x}$ and $S_{y y}$, have the same values as the perpendicular normal stresses, $S_{z z}$. They remain approximately at these levels also in the interfacial regions (see Figure 61a). Only a minor $z$-dependence can be recognised. When approaching an interface, the perpendicular normal stresses, $S_{z z}$, slightly decrease at the hot and increase at the cold thermostated region. They thus produce a contribution to the $z$-forces there that always acts so as to retard the roll cells. It must, in turn, be cancelled by a $y$-gradient of the shear stresses, $S_{z y}$. Otherwise, the sum of the local forces would not be zero as required above. For the sake of brevity, we do no check this explicitly. Instead, we would like to call the attention to the different roles of the normal stresses. On one hand, they promote, via $S_{y y}$ as function of $y$, and on the other hand, they retard, via i.e. $S_{z z}$ as function of $z$, the roll cells. The inverse applies to the shear stresses, $S_{y z}$ and $S_{z y}$. Such a dichotomy can be seen as an argument against the normal stresses driving the thermocapillary convection. We would then expect them to decrease all along the flows in the phases, as suggested by the stream filament theory or the theory of potential flows [spurk1996], for instance. Both theories apply, in our opinion at least approximately, to the outermost regions of a roll cell where friction plays only a minor role.

Concluding this section, we would like to underline again the similarity of the stress distribution in all our systems. It applies as well to the expanded systems from section 5.4.1, even if they have regions without flows. Thus, the shear stresses, $S_{y z}$ for instance, increase approximately linearly from one interfacial region to the other one in the system N-Ar5Ar5-0.6-1.0-13384-140964.74x8.00x37.6-100-140 (see Figure 63). Nevertheless, convection ceases between them (see Figure 
44). This deviation from the often linear relation between velocity gradients and shear stresses might indicate that we are dealing with an instability there. The $z$-gradient of these shear stresses is, of course, balanced by a $y$-gradient of the normal stresses, $\partial S_{y y} / \partial y$, again. 


\subsection{Thermocapillary Convection in the Unary Inferfacial Systems}

We considered succinctly, as a complement to our main studies of the liquid-liquid interfaces above, a few other ones. The comparisons below will yield interesting results. Thus similar interfacial flows occur also in liquid-vapour and solid-liquid systems. They were simulated using the same code as the liquid-liquid ones before. We shall not discuss in detail how far these new systems can be considered to be realistic, e.g. in terms of the ratio between the mean free path in the gas phase and the system dimensions or in terms of the assumed linear wall potentials, but look only for common characteristics. Thus, the flows at liquid-liquid and liquid-gas interfaces, for instance, point typically in the same direction, i.e. from hot to cold. We will then compare the conditions in the different systems to identify the common properties that might drive the interfacial flows.

Two important similarities will come to mind when comparing a nonequilibrium liquid-liquid and a liquid-vapour system (see section 5.6.1). On one hand, there are, in direction of the temperature gradient (the $y$-direction in our systems), density gradients for the majority and minority components. On the other hand, the interfacial particles feel a force which, on the average, tends to drag them into the liquid phase at liquid-gas interfaces or into the one where they constitute the majority at liquid-liquid interfaces.

The similarities between liquid-liquid and liquid-vapour interfaces exist also in comparison to solidfluid interfaces with, as we call it here, inverse thermal creep. It runs, as the thermocapillary convection, from hot to cold. Before going go to the results, we shall first distinguish here regular and inverted thermal creep again to prevent misunderstandings, see part $\mathbf{2}$ for more details. Solidfluid interfaces involve a so-called wall potential which describes the interactions between the particles in the adjacent phases. It is typically attractive-repulsive so that an adsorption layer forms. Then, creep flows, typically directed from cold to hot, occur in a lateral temperature gradient. J.C. Maxwell explained them in the case of solid-dilute gas interfaces. His explanation uses the diffusive part in the reflection of the incident gas particles on the adsorption layer (see section 2.3.1). 
Wold et al. [wold1999] simulated thermal creep at higher fluid phase densities. They make it hard to distinguish the adsorption layer. Nevertheless, the results clearly show how its diffusive character rather retards the flows than driving them at such densities. Different from the authors' other simulations described so far, an additional one uses a purely repulsive wall potential. As a result, the creep inverts. This inverted creep could be related to the thermocapillary convection found at liquid-fluid interfaces, which are at the focus of our work.

To be able to study the inverted creep flows in more detail, we reproduced them in a simpler solidfluid system than the one used by Wold et al. (see section 5.6.2). We will see in it the same relation between the density $y$-gradient and the com $y$-velocity as in our liquid-liquid and liquid-vapour systems. The interfacial mean forces per particle - in the solid-fluid system, they include the internal interactions among the particles as well as the repulsive external interactions with the wall - are also similarly distributed at the three different interfaces. Given this similarity we shall assume a relationship. All these flows have a common underlying effect which continues to be called "thermocapillary" here. Since particularly the occurrence of the inverted thermal creep depends on the type of the wall potential, we then studied its influence on the convection in more detail. It responds hardly to our manipulations of the force field.

If we make the repulsive wall permeable, however, and allow, in addition to the repulsive interactions with it, interactions with another liquid phase on the other side, the flows weaken clearly (see section 5.6.3). Such a setup strongly resembles in many aspects the previously studied binary liquid-liquid systems. We subsequently applied, apart from the repulsive permeable wall, also other external force fields. If they are attractive instead of repulsive the thermocapillary convection inverts. It can even cross the "interfaces" if we apply a force field that always drags the particles away from the origin of the coordinate system. Although such systems may lack a physical example, they show clear relations between the mean forces per particle, be they externally affected or purely self-imposed by the formation of two distinct phases, the density distribution and the interfacial flows. Further research into them should, in our opinion, focus on these relations on the molecular level. There is possibly even a way to describe them analytically. We found thermocapillary convection even in 2D MD simulations of binary liquid-liquid interfaces (see part 12). Thus, one dimension could likely be omitted, which would simplify such an analytical approach. For the sake of brevity, we do not go into the details of 2D simulations here. 


\subsubsection{Liquid-Vapour Interfaces}

We simulated liquid-vapour nonequilibrium interfacial systems in addition to our liquid-liquid ones. Both have a great deal in common, which may tell us about the local conditions for the occurrence of the thermocapillary effect. Since the liquid-vapour interfaces arrange, like the liquid-liquid ones, so as to minimise their surface area, see also part 11, we could to a large extent reuse our simulation setup from chapter 4.1. In order to keep the simulation system simple, only particles of the type Ar5 were considered. We placed 8192 of them arbitrarily inside a periodic cell of the dimensions $4.74 \times 8.0 \times 70.0 \mathrm{~nm}$ and simulated until a single liquid phase had occurred. Afterwards, we ran further steadying simulations of another six million time steps (30 ns) to verify its stability and finally production simulations of fifteen million time steps ( $75 \mathrm{~ns}$ ). In doing so, temperatures of $T_{C}=110 \mathrm{~K}$ and $T_{H}=130 \mathrm{~K}$, which lie well between the triple point and critical point temperatures of Ar5 (see Table 3), were maintained in the thermostated regions. This system shall be designated, on the basis of our previously introduced notation (see chapter 4.1), as N-Ar5-1.0-8192-4.74x8.00x70.0-110130.

We analysed the abovementioned unary liquid-vapour system almost in the same way as described for the binary liquid-liquid ones before (see chapter 4.3). In particular, the spatial resolution of the subvolumes was kept. It has proven adequate in our previous studies of the flows in liquid phases of comparable density. A subvolume in the gas-phase contains, however, on the average less than one particle, when using this resolution. Since we are, however, not interested in the gas phase, we refrain here from the statistical analysis which allowed us to find a suitable resolution (see section 4.3.4). We rather "worked around" the problem to keep program changes at a minimum. The determination of macroscopic flows is anyhow impossible in the gas phase due to the adverse ratio between the mean free path, which is according to the kinetic theory of gases [kennard1938] approximately on the order of $3 \mathrm{~nm}$ at the average system pressure and temperature, and the system dimensions. In order to avoid divisions by zero in the simulation code, we determined, different from our previous studies, only observables on the fly in a simulation, whose definition does not contain the local particle number in the denominator. Instead, their extensive counterparts were time averaged first, e.g. the kinetic energy in lieu of the temperature, the momentum in lieu of the com velocity, etc. After a simulation, we computed the intensive observables from the time averages of the extensive counterparts and the particle number. This procedure has already proven useful for the 
mean forces per particle in the liquid-liquid systems (see section 5.1.1). It does, however, not work for the standard deviations. We thus continued to compute them on-the-fly. Their result will be shown as "F", if the computation involved a division by zero.

Figure 64a shows the densities found in the liquid-vapour nonequilibrium interfacial system. We can clearly see the transition from the (only partially displayed) gas phase to the liquid one (L). The interfacial regions seem to exhibit higher heat transport rates that manifest themselves in the flatter temperature $y$-gradients (see Figure 64b). This manifestation results, as explained in section 5.2.2, from the unspecific allocation formula of our thermostats. Although the local conditions differ from those in the previously studied liquid-liquid systems, the liquid phase and its adjacent regions exhibit a similar convection pattern: It runs from hot to cold along the interfaces and the other way round away from them (see Figure 64c). The flows spread deeper into, or even slightly beyond, the interfacial regions, for two main reasons: On one hand, a smaller $z$-force separates the phases from each other (see Figure 64d,e). On the other hand, the gas phase has no sizeable opposing roll cell that could confine the one of the liquid phase. We shall thus assume that we are dealing with something very similar to a thermocapillary convection here. Spatially variable rates of evaporation and condensation likely superimpose onto it, but were not determined. They exist at a very small pressure decrease from the cold to the hot thermostated region (see Figure 64f). We shall check the similarity of the flows additionally in the so far unconsidered solid-liquid systems.

For the sake of completeness, we briefly comment on the results of two other simulated liquidvapour systems, N-Ar5-1.0-8192-4.74x8.00x70.0-120-140 and N-Ar5-1.0-8192-4.74x8.00x70.0130-150. They differ from the one above only in terms of the thermostated temperatures. The preparational and production simulations as well as their analysis were conducted in exactly the same way. There is a diminishing of the flows. It comes with wider but weaker interfacial density variations. The mean forces per particle decrease accordingly. All of the above changes are in agreement with those found in liquid-liquid systems for the increase of the average system temperature (see Figure 65). 


\subsubsection{Solid-Fluid Interfaces}

Wold et al. [wold1999] found inverted thermal creep flows at solid-fluid interfaces with a purely repulsive wall potential (see chapter 5.6). We reproduced such flows, which strongly resemble the thermocapillary convection at liquid-liquid and liquid-gas interfaces, in a different simplified system. It reuses to a large extent the simulation setup of our liquid-vapour systems from the preceding section. We additionally confined the particle movement in $z$-direction by applying an additional force, $\mathfrak{l}_{z, f f 1}$, on the particles. It increases linearly with a slope of $s_{f f 1}$ beyond a certain distance, $z_{f f l}$, from the origin (see Figure 66). Both the onset and the increase of this force must be chosen so as to reproduce essentially insurmountable walls. Particles that "collide" with them are specularly reflected.

We positioned $N=6870$ Ar5 particles arbitrarily in between two such walls with a distance of $9.4 \mathrm{~nm}$, i.e. $z_{f f l}= \pm 4.7 \mathrm{~nm}$. The external force increases with a slope of $s_{f f l}=-10 \mathrm{pN} / \mathrm{nm}$, which is roughly as steep as the maximum slope of the internal particle force in the Ar5Ar5 liquid-liquid reference system, N-Ar5Ar5-0.6-1.0-3346-3524-4.74x8.00x9.40-100-140, from chapter 5.4. The system dimensions were $4.74 \times 8.00 \times 50.0 \mathrm{~nm}$, of which the last one was simply chosen large enough to avoid particle interactions across the periodic boundaries. We include all the underlying parameter values above, as in previous cases, into a brief denomination of the simulation: N-Ar5-1.0-6870$4.74 \times 8.00 \times 50.0-100-140-\mathrm{ff} 1 \sim 4.70 \sim-10$. Since the total number of particles and their accessible volume also resemble those in the above-mentioned Ar5Ar5 reference system, we can expect similar densities. They are much higher than in the studies by Wold et al. . We willingly chose, different from them, a very simple spatial dependence of the external force to explore the general validity of their findings. The production run includes 15 million time steps ( $75 \mathrm{~ns})$. It continues a steadying simulation of 6 million time steps (30ns) which confirmed that our nonequilibrium solidliquid system had assumed a stationary state. We analysed the results in almost the same way as explained for our liquid-liquid systems. The large fluctuations, that likely occur due to the low densities at the walls, come with divisions by zero. We thus proceeded as described in the section above. Moreover, we consider and display the values of the observables only for subvolumes that contain, on the average, at least one particle. Particularly the $z$-velocity components lend themselves for judging the intensity of the convection since they have their maxima remote from the interfaces. 
Figure $67 \mathrm{a}$ shows the densities in the nonequilibrium solid-fluid system. We can clearly see that they decrease at the repulsive walls. The additional interactions with them manifest themselves also in the mean forces per particle (see Figure 67d,e). The ones in $z$-direction, for instance, increase at first more strongly before assuming a linear variation remote from the liquid phase. Despite our simplifications, i.e. the linearly increasing external forces and the "specular" wall collisions, an inverse creep occurs as in the studies by Wold et al. (see Figure 67c). Its occurrence can thus be considered confirmed for a broader range of situations. The flows run, like in our other interfacial systems before, from hot to cold in the region with the steeper density $y$-gradients and the other way round where they are flatter. We shall thus assume that, due to the similarity in the convection pattern and its underlying local conditions, that all these flows are related to each other, i.e. they result from a common effect, no matter what type of interface. We shall continue to call them "thermocapillary". Figure $67 \mathrm{~b}$ shows, for the sake of completeness, the temperature distribution.

We verified up to here that the thermal creep can invert as function of the particle-wall interactions. Its details are still unknown. Some sort of "transition interval", should exist, however, in which only slight changes to the particle-wall interactions result in this inversion. It is also unknown to what extent they affect the creep flows remote from such a transition region. A better understanding of their dependency could even help understand the role of the mean forces at liquid-fluid interfaces. Hence, we systematically varied in additional simulations of the solid-liquid system from above the slope of the external forces. The results will show that the smaller it is, the more the creep flows intensify to our surprise (see Figure 68). Their intensification contradicts, at first sight, our previous findings at liquid-fluid interfaces. They involved e.g. in sections 5.4.4 or 5.6.1 a more intense convection, the wider the interface and thus the stronger the mean force per particle is.

The unexpected response of the convection to our manipulations of the external force field "ff" might also be caused by the different volume accessible to the particles. Remember how lowering it favoured more intense flows in previous studies (see section 5.4.1). Hence, we attempted to compensate for this in additional simulations by readjusting the system $x$-dimension. Such manipulations should not disturb the flows since it extends along the rotation axis of the roll cells. We determined suitable dimensions in $x$-direction by adjusting them iteratively in successive preparational simulations until the liquid bulk phase had approximately the same particle density as in the Ar5Ar5 nonequilibrium liquid-liquid system. Each preparational simulation was 6 million time steps (30ns) long while the subsequent production simulations covered 15 million time steps (75ns). The solid-fluid interfacial system responds, despite these compensations, in the same 
qualitative way as without them (see Figure 68). We thus consider the found promotive influence, that a slower increase of the external forces has, on the convection as a physical fact.

Note, in addition to the dependence on the slope of the external force field, also the generally much higher intensity of the flows (see Table 30). It exists even if we additionally adjust the $x$-dimension of the solid-liquid system such that its liquid phase has approximately the same density as in liquidfluid systems with a liquid phase of similar size. Compare, for instance the systems

N-Ar5Ar5-0.6-1.0-6692-7048-4.74x8.00x18.8-100-140

and N-Ar5-1.0-6870-4.18x8.00x50.0-100-140-ff1 4.70 -10.

Apparently, not the stronger spatial variation at a liquid-fluid interface thus favours the flows. They rather have a maximum in the absence of particle exchanges between both phases and decrease, the stronger they are. Hence, measures were taken to allow for some exchange in further studies. We simply divided an unary liquid phase into two by applying a suitable external force field. As a result, the setup has concurrently some features of the previously studied binary liquid-liquid systems. See the next section for more details.

If smaller interfacial forces favour stronger flows, as seen in the solid-fluid systems above, then why are the flows at wide liquid-liquid interfaces weaker than at thin ones, as established for instance in sections 5.2.1 or 5.4.4. The answer is likely that the exchange of particles between both liquid phases decelerates the flows. This would also explain why there is a much more intense convection in our solid-fluid systems, which lack such an exchange, than in any of the liquid-liquid systems simulated by us (see Table 30). Take for instance, the liquid-liquid system,

N-Ar5Ar5-0.6-1.0-6692-7048-4.74x8.00x18.8-100-140.

Each of its liquid phases has approximately the same dimensions as the one in the solid-fluid system, N-Ar5-1.0-6870-4.18x8.00x50.0-100-140-ff1 4.70 -10. Moreover the interfacial widths are comparable. Nevertheless, the convection in the phases of the liquid-liquid system is only a fifth as intense as in the liquid phase of the solid-fluid system. Hence, we took measures to allow for some exchange in further studies. We simply divided an unary liquid phase into two by applying a suitable external force field. As a result, the setup has concurrently some features of the previously studied binary liquid-liquid systems. See the next section for more details. 


\subsubsection{Liquid Phases Separated by an External Force}

As indicated in the previous section, our focus lies on the effective interaction between two adjacent fluid phases, which comprises also the exchange of particles between them. Such interactions turned out to decelerate the flows in comparison to a related solid-fluid system with specular wall reflections (see section 5.6.2). To study in more detail the role of this interaction, we designed a system of a pure liquid that is separated into two phases by "interfacial regions". In them, a local external force, $\mathbf{l}_{f f 2}$, acts on each particle. Particles that enter one of these regions on either side are driven back into the direction where they came from. Nevertheless, the force is weak enough that some particles manage to pass. We manipulated its strength to study the role of the effective particle interaction between both phases.

Figure 69 plots the spatial variation of the external force. Its absolute $z$-component has an absolute maximum of $i_{z, f f 2}=10 \mathrm{pN}$ at $z= \pm 2.0 \mathrm{~nm}$ and decreases on its both sides over a distance of $1.0 \mathrm{~nm}$ to zero, i.e. $\mathfrak{z}_{z, f f 2}=0 \mathrm{pN}$ at $z_{l b}= \pm 1.0 \mathrm{~nm}$ and at $z_{u b}= \pm 3.0 \mathrm{~nm}$. We simply customised the solid-liquid system from the previous section accordingly. Thus, the $z$-dimension was reduced back to a length of $9.4 \mathrm{~nm}$ in order to maintain the liquid density. All other parameter values remained. We even conducted the preparational and production simulations as well as the analysis of the results in exactly the same way. The name of the system includes, as in previous cases, its parameter values, i.e. N-Ar5-1.0-6870-4.74x8.00x9.40-100-140-ff2 1.0 3.0 10, to help distinguish it from other ones.

We show in Figure 70a the density distribution. A clear depletion exists where the external force is non-zero. It contributes together with the interatomic forces to the mean field seen in Figure 70d,e. Figure $70 \mathrm{~b}$ shows the temperature distribution. The convection (see Figure 70c) is only half as intense as in the comparable solid-liquid systems from section 5.6.2 (see Table 30). It weakens even more, if we reduce the slope of the external force (see Figure 71),

N-Ar5-1.0-6870-4.74x8.00x9.40-100-140-ff2 1.0 3.0 1

and N-Ar5-1.0-6870-4.74x8.00x9.40-100-140-ff2 1.0 3.0 100.

Transferring these findings to liquid-liquid interfaces, we suspect that the effective interaction between both phases, which comprises also the exchange of particles, must retard the 
thermocapillary convection. This interaction increases with decreasing variation of the interfacial mean forces. As a result, we find weaker flows at wider interfaces. The interaction between both phases could also explain our results in section 5.2.2. They are on the Ar1Ar2 interfacial systems with altered ArB particle mass. Within each of them, both phases turned out to exhibit a convection of approximately the same intensity although their viscosity differs strongly.

For the sake of completeness, the flows in two additional systems with different spatial dependencies of the external force shall be considered here. Having studied the influence of a repulsive external force above, we now use an attractive one, $\mathbf{t}_{f f 3}$. Its spatial $z$-dependence is almost as that of $\mathbf{t}_{f 2}$ above except that the sign has changed (see Figure 72). Figure 73a shows how the particles accumulate where the external force is non-zero in the system,

N-Ar5-1.0-6870-4.74x8.00x9.40-100-140-ff3 1.0 3.0 10.

This force superimposes with the interatomic one to yield the distribution in Figure 73e. Concurrently, there is an inversion of the convection compared to the above simulations with $\mathbf{t}_{f f 2}$ (see Figure 73c). It now resembles the "regular" thermal creep found by Wold et al. [wold1999] at attractive-repulsive walls. The previously described relation between the density $y$-gradients and the com $y$-velocities remains valid, although we are dealing with inverted flows now. They still run from hot to cold where the lower, here next to the interfaces, and the other way round where the higher, here right at the centre of the interfaces, density $y$-gradients exist.

The fact, that the above-mentioned relation is valid both in regular and inverted interfacial flows hints at an important role of the density distribution for a general description of them. We demonstrated already in section 5.5.1 that an "intermediate" density distribution appears in the nonequilibrium liquid-liquid systems. It lies between the one expected from corresponding nonequilibrium one-phase and equilibrium interfacial systems. All deviations from these two limiting distributions may be unstable states that lead to the interfacial convection. Figure $73 \mathrm{~b}$ shows the temperature distribution. The intensity increases the steeper the slope of the force field is, N-Ar5-1.0-6870-4.74x8.00x9.40-100-140-ff3 1.0 3.0 1

and N-Ar5-1.0-6870-4.74x8.00x9.40-100-140-ff3 1.0 3.0 100, as seen previously in the simulations with $\mathbf{t}_{f f 2}$ (see Figure 74). This behaviour suggests new approaches to study the thermocapillary effect. We shall come back to them at the end of this section. 
In addition to the purely repulsive and attractive external forces before, we implemented a mixture of both, referred to as $\mathbf{l}_{f f 4}$. Its $z$-component increases in absolute terms as well from the lower, $z_{l b}= \pm 1.0 \mathrm{~nm}$, and the upper, $z_{u b}= \pm 3.0 \mathrm{~nm}$, boundaries to an extreme value of $l_{\mathrm{z}, \mathrm{ex}}=1 \mathrm{pN}$, at the centre between them. The direction of the force is, however, such that it always drives the particles away from the origin of the coordinate system (see Figure 75). One could thus consider this force either as attractive with respect to the (thin) phase located at the origin or as repulsive with respect to the other (dense) phase. Figure 76a shows the resulting density distribution in the system N-Ar5-1.06870-4.74x8.00x9.40-100-140-ff4 1.0 3.0 1. We can clearly distinguish the phases on the different sides of the "interfaces". The convection now crosses them, i.e. it runs from hot to cold in the phase located at the origin and the other way round in the other phase (see Figure 76c). Such flows appear rather unusual at first sight, but their direction still remains, in the previously described way, related to the relative magnitude of the density $y$-gradients. For the sake of completeness, we show the distributions of the temperatures and forces in Figure 76b,d,e.

In our opinion, further research into interfacial flows should focus strongly on the relations between the different microscopic observables. Take, for instance, the above one between the density gradients and the flow direction. There is still little known about the stability of the density distributions and their response to forces, be they external as at solid-fluid or intrinsic as at liquidfluid interfaces. 


\section{Summary}

We explained the dilemma with the phenomena at solid-fluid and liquid-fluid interfaces previously (see part 1). They play an important role in many scientific and technological applications. The interfacial behaviour itself is, however, most of the time only poorly understood, mainly due to the difficult experimental accessibility. After all, the interfaces measure typically only a few Angstroms in width. We used a new simulation approach to gain insights. On the one hand, simulations allow, in principle, distortion-free "measurements". Our chosen molecular dynamics method, in particular, works additionally on a more fundamental level than the common continuum mechanical approaches. Hence, the simulated matter should exhibit the interfacial phenomena by "nature", i.e. there is no need to choose suitable material equations beforehand, as in continuum mechanics.

Capillary convection is presumably the most intriguing among all interfacial phenomena. Hence, we made particularly it subject of our studies. Due to the small number of particles computable, there were, however, doubts at first, if MD simulations can reproduce such a convection at all. As a result, we started out simple and focused on the thermocapillary convection. Its underlying temperature gradient is relatively easy to implement by manipulation of the particle velocities at the system boundaries (boundary driven MD simulations). Diffusocapillary convection, on the other hand, requires more difficult measures, such as the exchange of particles. The same holds for other kinds of capillary convection. Despite our focus, their understanding can likely benefit as well from our studies due to the close relationship between the phenomena.

In addition to the kind of capillary convection, we limited ourselves to studies of it at liquid-liquid interfaces. Both phases exhibit at least approximately the same properties at such interfaces. We could thus easily analyse the local behaviour of a system by using equally sized control volumes throughout it. Moreover, there was already a great deal of experience available from the literature. Two partially miscible liquids generally separate so as to minimise their interfacial area. Given both phases have approximately the same volume, the stable separated state exhibits two flat interfaces in a system with periodic boundaries. These interfaces lie parallel to the smallest surfaces in a rectangular cuboid simulation cell. We took advantage of such a separation for our purposes. A temperature gradient along the interfaces could be established by thermostating two separate slabs, 
that lie parallel to two opposite cell surfaces, to different temperatures. As explained previously in section 4.1.4, the thermostating was achieved by manipulation of the particle velocities.

As far as the substances are concerned, we sticked to our above-mentioned intention to start out simple. Binary mixtures of Argon-like Lennard-Jones (abbr. LJ) particles were chosen. If the repulsion between unlike ones of them is increased by an additional factor, they exhibit the required partial miscibility. We chose conservative parameter values and technical simplifications in the first simulations of a system described up to here. It was called nonequilibrium interfacial system (abbr. NEIS). Surprisingly, flows truly occurred in it, regardless of all our conservatism. Their exact cause was, however, still unclear. After all, they might have just as well been artefacts. Hence, we compared our found convection pattern to the one to expect from experiments. Afterwards, we devised in a thought experiment what the distribution of the other determined observables, e.g. densities or temperatures, must look like. It can be estimated by superimposing the much better understood behaviour in separate cut-outs of the nonequilibrium interfacial system:

- the phases being subject to the temperature gradient; Each of them constitutes a nonequilibrium one-phase system (abbr. NEOPS).

- several slabs perpendicular to the temperature gradient; Each of them is so thin that it resembles an equilibrium interfacial system (abbr. EIS).

The abovementioned observables turned out to be almost entirely distributed according to our expectations. Moreover, convection was found in additional simulations of corresponding nonequilibrium one-phase and equilibrium interfacial systems by using the same code. We are thus convinced of dealing with thermocapillary convection here. Subsequent simulations of similar systems further substantiate our conviction, but a terminal proof remains open.

Although we may have established the thermocapillary convection, the approximation of reality by the system behaviour could still suffer from the technical simplifications. Thus, there are minor, but noticeable, anomalies in the temperature distribution, for instance. It exhibits jumps at the thermostated regions. Moreover, the temperature gradients vary between both phases if they feature different thermal conductivities. We therefore checked several promising variants to reduce such anomalies by gradually adjusting the setup and the technical simplifications in additional simulations. Our adjustment of the cut-off radius or the thermostating algorithm resulted in hardly any improvements. Both were already chosen very carefully from the literature. We could, however, avoid specifically the variable temperature gradients between both phases by giving both 
substances the same LJ parameters for the interactions of like particles. A choice like that is, admittedly, unorthodox but not physically wrong. Moreover, it does not interfere with our goal to study the thermocapillary effect in principle, i.e. free from a certain mixture. Taking into account the adjustments above, a plausible analysis of it in further simulations thus seems possible. We called the improved systems simulated "homophasic" to distinguish them from the "heterophasic" ones simulated before.

We proceeded by systematically checking the influence of various physical system parameters, i.e. ones that do not relate to the simulation method itself but also have a physical meaning instead, e.g. the thermostated temperatures or the particle numbers, on the thermocapillary convection. Its intensity increases with increasing temperature gradient, with decreasing viscosity, and with decreasing interfacial width. These influences are in agreement with experimental results. The dependence on the system dimensions is much more interesting. Note, they were always varied together with the particle numbers so as to maintain the overall system densities. First, the convection does not spread arbitrarily deep into the phases if we increase the distance between the interfaces. Instead, it remains associated with them, which confirms again that we are truly dealing with an interfacial phenomenon here. The maximum spatial extent of the flows comes with an upper limit of their intensity. Second, if we extend the system in direction of the temperature gradient, and maintain its level at the same time, the flows intensify as well up to an upper limit. Their response results from the decreasingly important role of the turnaround flows at the thermostated regions. Finally, the intensity remains almost unaffected, if we extend the system simultaneously in both directions while keeping the temperature difference. Such a scaling behaviour could explain why the flow velocities seen in the simulations are of the same order of magnitude as in experiments. They have temperature differences comparable to ours but much larger length scales.

We studied the homophasic systems once again in a more sophisticated way, based on the insights gained in the variations of their physical parameters. The studies focus on the individual processes involved in the thermocapillary convection. Thus, we establish an intermediate density distribution in the nonequilibrium interfacial systems that lies between two limiting cases:

- a distribution devised from several related equilibrium interfacial systems; They all have the same pressure as the guiding system but different local temperatures from it.

- a distribution to expect from several related nonequilibrium one-phase systems. They all have the same pressures and local temperatures as the guiding system but different ones of its local 
compositions. Since particularly the interfacial compositions cannot be reproduced in bulk systems, we had to extrapolate the concentration dependence from them to obtain at least rough estimates.

Such an intermediate density distribution comes with a relative motion of the components in the flows.

Moreover, we determined the different "parts" of the local volume force in a nonequilibrium interfacial system. They are the individual summands obtained in computing the stress tensor divergence. We established for selected examples that these parts add to zero as required for the small Reynolds numbers in our systems. It is, however, invalid at the interfaces to associate in the interfacial region, as it is commonly done in the bulk, the shear stresses with friction and the normal stresses with pressure. Think only of their anisotropy in equilibrium systems, for instance. The balance between the different parts of the force raises doubts if the thermocapillary effect can be ascribed to one of both types of stresses at all. If so, our results militate against the common conception of the normal stresses, which also enter into the definition of the interfacial tension, as the driving force behind the thermocapillary convection. More likely, shear stresses start it as soon as a temperature gradient is applied to an initially equilibrium interface. As a result, gradients of the normal stresses build up. Further research into nonstationary systems might help answer these questions.

We additionally looked at the behaviour of liquid-gas and solid-liquid interfaces subjected to a lateral temperature gradient. They all exhibit the same convection pattern and share selected local conditions, such as the occurrence of mean forces per particle between neighbouring phases. We thus assumed the flows to result also from a common underlying effect. Its influences were studied in more detail by artificially reproducing various interface-like conditions in an otherwise bulk liquid. To do so we implemented several external force fields and allow for different interactions between both particles. A certain relation remains, nevertheless, valid in all simulations: The flows always run from hot to cold where the density varies only little with the temperature.

Our results show that MD simulations can contribute a lot to the research into the interfacial convection. They allow for monitoring its underlying processes on the microscopic scale. The relations between those properties, that are clearly defined down to it, deserve further study. 


\section{References}

Aa Y., Cao Z.H., Tang Z.M., Sun Z.W., 2005 Microgravity Sci. Tech. 17 (4) 5-13

$\mathrm{Hu}$ W.R.

Abramov Y.Y., Gladush G.G. 1970 Fluid Dyn. 11 (4) 193-200

Agrawal R., Kofke D.A. 1995 Mol. Phys. 85 23-42

Agrawal R., Kofke D.A. 1995 Mol. Phys. 85 23-42

Alexander K.F., Wirtz K. 1950 Zeitschrift für physikalische Chemie 195 165174

Allen M.P., Tildesley D.J. 2003 Computer Simulation of Liquids, Oxford University Press, Oxford

Andersen H.C. $1980 \quad$ J. Chem. Phys. 72 (4) 2384-2393

Anderson D.M., Mc Fadden G.B., 1998 Annu. Rev. Fluid. Mech. 30 139-165

Wheeler A.A.

Aoki K., Sone Y., Takeru Y. 1989 Physics Fluids A 1 (2) 409-419

Arya G., Chang H., Maginn E.J. $2001 \quad$ J. Chem. Phys. 115 8112-8124

Ashurst W.T., Hoover W.G. 1975 Phys. Rev. A 11 (2) 658-678

Atkins P., de Paula J. 2002 Atkins' Physical Chemistry 7th ed, Oxford University Press, Oxford

Bakanov S.P. 1977c Zhurnal Tekhnicheskoi Fiziki 47 421-427

[Soviet Physics - Technical Physics 22 (2) 1977 248-251]

Bakanov S.P. 1991 J. Aerosol Sci. 22 (Suppl. 1) S215-S218

Bakanov S.P. 1992 Uspekhi Fizicheskikh Nauk 162 133-152, Soviet Physics Uspekhi 35 (9) 783-792

Bakanov S.P. 1995 J. Aerosol Sci. 26 (1) 1-4

Bakanov S.P., Derjaguin B.V., 1977b J. Coll. Interf. Sci. 60 (1) 172-176

Roldughin V.I.

Bakanov S.P., Deryagin B.V., Roldugin 1976 Doklady Akademii Nauk SSSR 227 329-332

V.I. [Soviet Physics - Doklady 21 (3) 152-154]

Bakanov S.P., Roldughin V.I. 1977a Kolloidnyi Zhurnal 39 (6) 1027-1038

[Colloid Journal of the USSR 36 (6) 1977 907917]

Beddard G. 1993 Rep. Prog. Phys. 56 63-171

Bénard H. 1900a Revue Générale des Sciences Pures et Appliquées, tome 11, 1261-1271

Bénard H. 1900b Revue Générale des Sciences Pures et Appliquées, tome 11, 1309-1328

Bénard H. 1901 Ann. Chim. Phys., 7ième série, tome 23, 62-144 
Bendat J.S., Persol A.G. 2000

Benjamin I. 1997

Berendsen H.J.C., Postma J.P.M., van 1984 Gunsteren W.F., DiNola A., Haak J.R.

Beresnev S.A. 1993

Beresnev S.A., Chernyak V.G. 1985

Berne B.J., Harp G.D. 1970

Beveridge D.L., DiCapua F.M. 1989

Billes W., Bazant-Hegemark F., Mecke 2003 M., Wendland M., Fischer J.

Binder K., Müller M. 2000

Binnig G., Quate C.F., Gerber Ch. 1986

Bird R.B., Stewart W.E., Lightfoot E.N. 2002

Block M.J. 1956

Bopp P.A., Buhn J.B., Maier H.A., 2008 Hampe M.J.

Bordat P., Müller-Plathe F. 2002

Bordat P., Reith D., Müller-Plathe F. 2001

Bougie J., Kreft J., Swift J.B., Swinney 2005

H.L.

Bougie J., Moon J.S., Swift J.B., 2002 Swinney H.L.

Braunsfurth M.G., Homsy G.M. 1997

Brock J.R. 1962

Brock J.R. 1967a

Brock J.R. 1967b

Brock J.R. 1968

Buhn J.B. 2004a

Buhn J.B., Bopp P.A., Hampe M.J. 2004b

Buhn J.B., Bopp P.A., Hampe M.J. 2006

Byers R.L., Calvert S. 1969

Cahn J.W., Hilliard J.E. 1958

Cahn J.W., Hilliard J.E. 1958
Random Data: Analysis and Measurement Procedures 3rd ed, John Wiley \& Sons, New York

Annu. Rev. Phys. Chem. 48 407-451

J. Chem. Phys. 81 (8) 3684-3690

J. Aerosol Sci. 24 (Suppl. 1) S183-S184

Doklady Akademii Nauk SSSR 285 1377-1381 [Soviet Physics - Doklady 30 (12) 1985 10551057]

Adv. Chem. Phys. 17 130-132

Annu. Rev. Biophys. Bio. 18 431-492

Langmuir 19 10862-10868

Int. J. Mod. Phys. C 11 1093-1114

Phys. Rev. Lett. 56 (9) 930-933

Transport Phenomena 2nd ed, John Wiley \& Sons, New York

Nature 178 (4534) 650-651

Chem. Eng. Commun. 195 (11) 1437-1464

J. Chem. Phys. 116 (8) 3362-3369

J. Chem. Phys. 115 8978-8982

Phys. Rev. E 71021301

Phys. Rev. E 66051301

Phys. Fluids 9 (5) 1277-1286

J. Coll. Interf. Sci. 17 768-780

J. Coll. Interf. Sci. 23 448-452

J. Coll. Interf. Sci. 25 392-395

Phys. Fluids 11 (4) 922-923

Molekulardynamische Untersuchungen einfacher Flüssig-flüssig-Phasengrenzsysteme, Dissertation, Darmstadt

Fluid Phase Equilibr. 224 (2) 221-230

J. Mol. Phys. 125 187-196

Ind. Eng. Chem. Fundamen. 8 (4) 646-655

J. Chem. Phys. 28 (2) 258-267

J. Chem. Phys. 28 258-267 
Callen H.B. 1985 Thermodynamics and an Introduction to

Thermostatistics 2nd ed., John Wiley and Sons, New York

Cardin P., Nataf H.C. 1991b Europhys. Lett. 14 (7) 655-660

Cardin P., Nataf H.C., Dewost P. 1991a J. Phys. II 1 599-622

Carpick R.W., Salmeron M. 1997

Cerisier P, Occelli R., Pantaloni J. 1988

Chandler D. 1987

Chang Y.C., Ranade M.B., Gentry J.W. 1995

Chapman S., Cowling T.G. 1970

Chun C.H., Wuest W. 1979

Chun C.H., Wuest W. 1982

Cicotti G., Ferrario M., Hynes J.T., 1989 Kapral R.

Cicotti G., Tenenbaum A. 1980

Cieplak M., Koplik J., Banavar J.R. 2006

Cieplak M., Koplik J., Bavanar J.R. 1999

Cieplak M., Koplik J., Bavanar J.R. 2000

Cohen E.G.D. 1993

Colinet P., Legros J.C., Velarde M.G. 2001

Coopey M., Tourbez H., Valat P., 1980 Alpert B.

Cramer A., Metzger J., Schwabe D., 1995 Scharmann A.

Crookes W. 1874

Crookes W. 1876

Crookes W. 1880

Croxton C.A. 1975
Chem. Rev. 97 1163-1194

in Velarde M.G., Physicochemical

Hydrodynamics - Interfacial Phenomena, NATO

ASI Series, Series B: Physics 174, Plenum

Press, New York, 1988, 199-208

Introduction to Modern Statistical Mechanics, Oxford University Press, New York

J. Aerosol Sci. 26 (3) 407-428

The Mathematical Theory of Non-Uniform Gases, Cambridge University Press, Cambridge

Acta Astronaut. 6 1073-1082

Acta Astronaut. 9 (4) 225-230

Chem. Phys. 129 241-251

J. Stat. Phys. 23 (6) 767-772

Phys. Rev. Lett. 96114502

Physica A 274 281-293

Physica A 287 153-160

Physica A 194(1-4) 229-257

Nonlinear Dynamics of Surface-Tension-Driven Instabilities, Wiley-VCH, Berlin

Nature 284 (4) 568-570

in Sahm S.R., Keller M.H., Schiewe B. (Eds.), Proceedings on the Norderney Symposium on Scientific Results of the German Spacelab Mission D-2: March 14-16 1994, 1995, Wissenschaftliche Projektführung D-2 RWTH Aachen, 259-271

Philos. T. R. Soc. Lond. (1776-1886) 164 501527

Philos. T. R. Soc. Lond. (1776-1886) 166 325376

Philos. T. R. Soc. Lond. (1776-1886) 170 87134

Introduction to Liquid State Physics, John Wiley \& Sons, London 
Darling G.R., Holloway S. 1995

Daviaud F., Vince J.M. 1993

Davis L.A. $1975 \mathrm{a}$

Davis L.A. $1975 b$

Davis S.H. 1987

De Groot S.R. 1960

De Saedeleer C., Garcimartin A.,

Chavepeyer G., Platten J.K.

Delhommelle J., Millie P. 2000

Denbigh K.G., Raumann G. 1952a

Denbigh K.G., Raumann G. 1952b

Derjaguin B.V., Bakanov S.P. $1962 b$

Derjaguin B.V., Storozhilova A.I., 1966 Rabinovich Y.I.

Derjaguin B.V., Yalamov Y. 1965

Deryagin B.V., Bakanov S.P. 1961

Deryagin B.V., Bakanov S.P. 1962a

Deryagin B.V., Yalamov Y.I., Ivchenko 1967 I.N.

Dirac P.A.M. 1999

Drazin, P. G. 2002

Drazin, P.G., Reid, W.H. 1981

Dunn J.E., Serrin J. 1985

Dupont O., Queeckers P., Legros J.C. 1995

Dwyer H.A. 1967

Eckert K., Bestehorn M., Thess A. 1998

Eilert A. 1914

Epstein P.S. 1929

Evans D.J. 1982
Rep. Prog. Phys. 58 1595-1672

Phys. Rev. E 48 (6) 4432-4436

J. Chem. Phys. 62 (6) 2278-2285

J. Chem. Phys. 62 (7) 2833-2839

Annu. Rev. Fluid Mech. 19 403-435

Thermodynamik Irreversibler Prozesse, Bibliographisches Institut, Mannheim

Phys. Fluids 8 (3) 670-676

Molec. Phys. 99 619-625

Proc. R. Soc. Lon. Ser. A 210 (1102) 377-387

Proc. R. Soc. Lon. Ser. A 210 (1103) 518-533

Nature 196 669-670

J. Coll. Interf. Sci. 21 35-58

J. Coll. Interf. Sci. 20 555-570

Doklady Akademii Nauk SSSR 141 (2) 384-386 [Doklady Akademii Nauk SSSR - Physical Chemistry 1411961 857-861]

Doklady Akademii Nauk SSSR 147 (1) 139-142 [Doklady Akademii Nauk SSSR - Physical Chemistry 1471962 778-782]

Doklady Akademii Nauk SSSR 173 (6) 12871290 ,

[Soviet Physics Doklady 12 (4) 362-364]

The Principles of Quantum Mechanics 4th ed, Oxford University Press, Oxford

Introduction to hydrodynamic stability, Cambridge University Press, Cambridge

Hydrodynamic stability, Cambridge University Press, Cambridge

Arch. Ration. Mech. An. 88 95-133

in S.R. Sahm, M.H. Keller, B. Schiewe (Eds.), Proceeding on the Norderney Symposium on Scientific Results of the German Spacelab Mission D-2 1994, DARA, Bonn, 243-246

Phys. Fluids 10 (5) 976-984

J. Fluid Mech. 356 155-197

Z. Anorg. Chem. 88 1-37

Z. Phys. 54 537-563

Phys. Lett. 91A (9) 457-460 
Evans D.J. 1986 Phys. Rev. A 34 (2) 1449-1453

Evans D.J., Morriss G.P. 1990 Statistical Mechanics of Nonequilibrium

Liquids, Academic Press, London

Ezersky A.B., Garcimartín A., Burguete 1993 Phys. Rev. E 47 (2)

J., Mancini H.L., Pérez-García C.

Feddersen W. 1872 Berichte d. Königl. Sächs. Gesellsch. d.

Wissensch. Mathem.-Phys. K1. 24. p. 49

Feddersen W. 1873 Annalen der Physik und Chemie 224 (2) 302311

Fermi E. 1937 Thermodynamics, Prentice-Hall, New York

Fick A.E. 1855 Ann. Phys. 170 (1) 59-86

Fincham D., Quirke N., Tildesley D.J. 1986 J. Chem. Phys. 84 (8) 4535-4546

Findlay, Alexander 1951 The Phase Rule And Its Applications 9th ed. , Dover Publications, New York

Fourier J.B.J. 1822 in Gabay J., Théorie analytique de la chaleur, J. Gabay, Paris, 1988

Frank S., Schwabe D. 1997 Exp. Fluids 23 234-251

Frenkel D., Smit B. 2002 Understanding Molecular Simulation, Academic Press, San Diego

Fresnel A. 1825a Ann. Chim. Phys. 29 57-

Fresnel A. 1825b Ann. Chim. Phys. 29 107-

Fuchs N.A. 1964 The Mechanics of Aerosols, Pergamon Press, Oxford, 1964

Fuchs N.A. 1982 J. Aerosol Sci. 13 (4) 327-330

Galliéro G., Colombani J., Bopp P.A., 2006 Physica A 361 494-510

Duguay B., Caltagirone J.P., Montel F.

Galliéro G., Boned C., Baylaucq A., 2005 Montel F.

Gauss C.F. 1830 Commentationes Societatis Regiae Scientiarum

Geysermans P., Elyeznasni N., Russier 2005 J. Chem. Phys. 123204711 V. Gibbs, J.W. 1875

Gilgen R., Kleinrahm R., Wagner W. 1994 Gillon P., Homsy G.M. 1996 Gottingensis Recentiores 7, Dieterich, Gottingae, Translation in Klassiker der exacten Wissenschaften 135, Engelmann, Leipzig

Fluid Phase Equilibr. 234 (1-2) 56-63

On the Equilibrium of Heterogoeneous Substances, Transactions of the Connecticut Academy III, (10)1875 - (5)1876 108-248, and (5)1877 - (7)1878 343-524 in The Scientific Papers of J. Willard Gibbs, Ox Bow Press, Connecticut, 1993, 55-353

J. Chem. Thermodyn. (26) 383-398

Phys. Fluids 8 (11) 2953-2963 
Glansdorff P, Prigogine I. 1971

Goldstein H., Poole C., Safko J. 2002

Gorelov S.L. 1976

Grew K. E., Ibbs T.L. 1952

Grodzka P.G., Bannister T.C. 1972

Grodzka P.G., Bannister T.C. 1975

Groß A. 1998

Haase R. 1963
Thermodynamic Theory of Structure, Stability, and Fluctuations, Wiley-Interscience, London

Classical Mechanics 3rd ed., Pearson, San Francisco

Fluid Dyn. 11 (5) 800-804

Thermal Diffusion in Gases, Cambridge

University Press, Cambridge

Science, 176 506-508

Science, 187 165-167

Surf. Sci. Rep. 32 (8) 291-340

Thermodynamik der irreversiblen Prozesse, Dr. Dietrich Steinkopff, Darmstadt

Haase R., De Greiff H.J. 1971 Z Naturforsch. A 26 1773-1774

Haase R., De Greiff H.J., Buchner J.H. 1970

Haase R., Greiff H.J. 1965

Z Naturforsch. A 251970 1080-1085

Haase R., Steinert C. 1959

Haberlandt R., Fritzsche S., Peinel G., 1995 Heinzinger $\mathrm{K}$.

Hadjiconstantinou N.G., Garcia A.L. 2003

Hafskjold B. 2002a

Z. Phys. Chem. Neue Fol. 44 301-313

Z. Phys. Chem. Neue Fol. 21 270-279

Molekulardynamik, Friedrich Vieweg \& Sohn, Braunschweig

J. Comput. Phys. 187 274-297

in W. Köhler, S. Wiegand (Eds.), Thermal Nonequilibrium Phenomena in Fluid Mixtures, Springer, Berlin, 3-23

Hafskjold B., Ikeshoji T. 2002b Phys. Rev. E 66011203

Hafskjold B., Ikeshoji T., Ratkje S.K. 1993

Mol. Phys. 80 1389-1412

Hafskjold B., Ratkje S. K. 1995

J. Stat. Phys. 78 463-494

Haile J.M. 1997

Hampe M.J. 1980

Han J.H., Sun Z.W., Dai L.R., Xie J.C., 1996 $\mathrm{Hu}$ W.R.

Hanley H.J.M. 1966

Hanley H.J.M., Steele W.A. 1965

Hannon L., Lie G.C., Clementi E. 1986

Hansen J.P., Verlet L. 1969

Harasima A. 1958
Molecular Dynamics simulation: Elementary Methods, John Wiley \& Sons, New York

Zur Thermodynamik der Transportprozesse in Grenzflächensystemen, Dissertation, Fakultät für Maschinenwesen, Technische Universität München

J. Cryst. Growth 169 129-135

T. Faraday Soc. 62 2395-2402

T. Faraday Soc. 61 2661-2670

Phys. Lett. A 119 174-177

Phys. Rev. A 184 151-161

Molecular Theory of Surface Tension in Prigogine I. (Ed.), Advances in Chemical Physics Vol. 1, Interscience Publishers, New York, 1958, 203-227 
Harvey S.C., Tan R. K. Z., Cheatham E. 1998 Haye M.J., Bruin C. 1994

Heffelfinger G.S., van Swol F. 1994

Heinz H., Paul W., Binder K. 2005

Hill, Terell L. 1986

Hill, Terell L. 1987

Hirschfelder J.O., Curtiss C.F., Bird 1966 R.B.

Hirshfeld D., Rapaport D.C. 1998

Hirshfeld D., Rapaport D.C. 2000

Hockney R.W., Eastwood J.W. 1988

Hoover W.G. 1985

Hu W.R., Shu J.Z., Zhou R., Tang Z.M. 1994

Humphris A.D.L., Miles M.J., Hobbs 2005

J.K.

Ikeshoji T. 2004
J. Comput. Chem. 19 (7) 726-740

J. Chem. Phys. 100 556-559

J. Chem. Phys. 100 7548-7552

Phys. Rev. E 72066704

An Introduction to Statistical Thermodynamics, Dover Publications, New York

Statistical Mechanics, Dover Publications, New York

The Molecular Theory of Gases and Liquids, John Wiley and Sons, New York

Phys. Rev. Lett. 80 (24) 5337-5340

Phys. Rev. E 61 (1) R21-R24

Computer Simulation Using Particles, Institute of Physics Publishing, Bristol

Phys. Rev. A 31 1695-1697

J. Cryst. Growth 142 379-384

Appl. Phys. Lett. 86034106

Molecular Dynamics Simulation and Local Quantities in Khin-Yong L., Heow-Puh L. (Eds.), Lecture Notes Series, Institute for Mathematical Sciences, National University of Singapore Vol. 6 - Computational Methods in Large Scale Simulation, World Scientific Publishing Company, Singapore, 2004

Ikeshoji T., Hafskjold B. 1994 Mol. Phys. 81 251-261

Imaishi N., Fujinawa K. 1974

H. Chem. Eng. Japan 7 (2)87-92

Irving J.H., Kirkwood J.G. 1950

J. Chem. Phys. 18 (6) 817-829

Ivchenko I.N., Loyalka S.K., Tompson 1993 R.V.

Ivchenko I.N., Yalamov Y.I. 1971b

Izvestiya Rossiiskoi Akademii Nauk Mekhanika Zhidkosti i Gaza 6 160-163 [Fluid Dynamics 28 (6) 1993 876-878]

Zurnal fiziceskoj chimii 45 (3) 577-582

[Russian Journal of Physical Chemistry 45 (3) 1971 317-319]

Ivchenko I.N., Yalamov Y.I., 1971a Russ. J. Phys. Ch. 45 (3) 1971 320-322 Rabinovich Y.I.

Jacobsen S., Brock J.R. 1965

J. Coll. Sci. Imp. U. Tok. 20 544-554

Jaynes E.T. 1980

Annu. Rev. Phys. Chem. 31 579-601

Joseph, D.D. 1976 a

Stability of fluid motions I (Tracts in Natural Philosophy 27), Springer, Berlin

Joseph, D.D. 1976b Stability of fluid motions II (Tracts in Natural Philosophy 28), Springer, Berlin 
Juel A., Burgess M., McCormick W.D., 2000 Physica D 143 169-186

Seift J.B., Swinney H.L.

\begin{tabular}{|c|c|c|}
\hline Jurisch M. & 1990 & J. Cryst. Growth 102 223-232 \\
\hline Kamotani Y., Kim J. & 1988 & J. Cryst. Growth 87 62-68 \\
\hline \multirow{2}{*}{$\begin{array}{r}\text { Kamotani Y., Ostrach S., Vargas M. } \\
\text { Keng E.Y.H., Orr C. }\end{array}$} & 1984 & J. Cryst. Growth 66 83-90 \\
\hline & 1966 & J. Coll. Interf. Sci. 22 107-116 \\
\hline Kennard E.H. & 1938 & $\begin{array}{l}\text { Kinetic Theory of Gases 1st ed., McGraw-Hill, } \\
\text { New York }\end{array}$ \\
\hline Kirkwood J.G. & 1946 & J. Chem. Phys. 14 (3) 180-201 \\
\hline Kirkwood J.G. & 1947 & J. Chem. Phys. 15 (1) 72-76 \\
\hline Kirkwood J.G. & $1949 \mathrm{a}$ & J. Chem. Phys. 17 (10) 988-902 \\
\hline Kirkwood J.G. & $1949 b$ & J. Chem. Phys. 17 (3) 338-343 \\
\hline Kjelstrup S., Hafskjold B. & 1996 & Ind. Eng. Chem. Res. 35 4203-4213 \\
\hline Knudsen M. & 1909 & Ann. Phys. 333 75-130 \\
\hline Knudsen M. & 1910 & Ann. Phys. 336 205-229 \\
\hline Knudsen M. & 1927 & Ann. Phys. 388 797-821 \\
\hline Kofke D.A. & 1993 & J. Chem. Phys. 98 4149-4162 \\
\hline Kollman P. & 1993 & Chem. Rev. 93 (7) 2395-2417 \\
\hline Kolmogorov A.N. & $1941 \mathrm{a}$ & Dokl. Akad. Nauk SSSR 30 299-303 \\
\hline Kolmogorov A.N. & $1941 b$ & Dokl. Akad. Nauk SSSR 32 19-21 \\
\hline Kolmogorov A.N. & 1991 & Proc. R. Soc. Lon. Ser. A 434 9-13 \\
\hline Koplik J, Banavar J.R. & 2006 & Phys. Rev.Lett. 96044505 \\
\hline Korteweg, D.J. & 1901 & $\begin{array}{l}\text { Archives Néerlandaises des Sciences Exactes et } \\
\text { Naturelles } 6 \text { 1-24 }\end{array}$ \\
\hline Koschmieder E.L. & 1988 & $\begin{array}{l}\text { in Velarde M.G., Physicochemical } \\
\text { Hydrodynamics - Interfacial Phenomena, NATO } \\
\text { ASI Series, Series B: Physics 174, Plenum } \\
\text { Press, New York, 1988, 189-198 }\end{array}$ \\
\hline Koschmieder E.L., Biggerstaff M.I. & 1986 & J. Fluid Mech. 167 49-64 \\
\hline Koschmieder E.L., Prahl S.A. & 1990 & J. Fluid Mech. 215 571-583 \\
\hline Koschmieder E.L., Switzer D.W. & 1992 & J. Fluid Mech. 240 533-548 \\
\hline Kroes G.J. & 1999 & Prog. Surf. Sci. 60 1-85 \\
\hline Kubo R. & $1957 \mathrm{a}$ & J. Phys. Soc. Jpn. 12 (6) 570-586 \\
\hline Kubo R., Yokota M., Nakajima S. & $1957 b$ & J. Phys. Soc. Jpn. 12 (11) 1203-1211 \\
\hline Landau L.D., Lifshitz E.M. & 2003 & $\begin{array}{l}\text { Course of Theoretical Physics: Vol. } 6 \text { Fluid } \\
\text { Mechanics 2nd ed; Butterworth-Heinemann, } \\
\text { Oxford }\end{array}$ \\
\hline Landau L.D., Lifshitz E.M. & 2007 & $\begin{array}{l}\text { Course of Theoretical Physics: Vol. } 5 \text { Statistical } \\
\text { Physics pt. } 1 \text { 3rd ed., Butterworth-Heinemann, } \\
\text { Oxford }\end{array}$ \\
\hline
\end{tabular}


Laplace P.S. 1880

Lee D.J., Telo da Gama M.M., Gubbins 1984

K.E.

Legros J.C., G. Pétré, Limbourg- 1984

Fontaine M.C.

Legros J.C., Limbourg-Fontaine M.C., 1988

Petre G.

Lemak A.S., Balabaev N.K. 1994

Levenstam M., Amberg G., Carlberg T., 1996 Andersson $\mathrm{M}$.

Levich V.G., Krylov V.S. 1969

LevichV.G. 1962

Leyssale J.-M., Vignoles G.L. 2008

Limbourg-Fontaine M.C., Legros J.C., $1986 a$ Pétré $\mathrm{G}$.

Limbourg-Fontaine M.C., Pétré G., 1986b Legros J.C.

Lippmann M.G. 1907

Loyalka S.K. 1969

Loyalka S.K. 1971a

Loyalka S.K. 1971c

Loyalka S.K., Cipolla J.W. 1971b

Loyalka S.K., Petrellis N., Storvick T.S. 1975

Ludwig C. 1856

Luettmer-Strathmann J. 2002

Luo H., Ciccotti G. et al. 1995

Lustig R. 1994a

Lustig R. 1994b

Lustig R. $1994 c$

Lyons K.B., Friedman J.M., Fleury P.A. 1978

Maitland G.C., Rigby M., Smith E.B., 1981 Wakeham W.A.

Marangoni C. 1871
Oevres complètes de Laplace vol 4, Gauthiers-

Villars, Paris

Mol. Phys. 53 (5) 1113-1130

Adv. Space Res. 4 (5) 37-41

in Velarde M.G., Physicochemical

Hydrodynamics - Interfacial Phenomena, NATO ASI Series, Series B: Physics 174, Plenum

Press, New York, 1988, 209-226

Mol. Simulat. 13 (3) 177-187

J. Cryst. Growth 158 224-230

Annu. Rev. Fluid Mech. 1 293-316

Physicochemical Hydrodynamics, Prentice-Hall, Eaglewood Cliffs

Chem. Phys. Lett. 454 299-304

Adv. Space Res. 6 (5) 35-39

Acta Astronaut. 13 (4) 197-208

Les comptes rendus hebdomadaires des séances de l'Académie des sciences 145 (7) 104-106

Phys. Fluids 12 (11) 2301-2305

Phys. Fluids 14 (1) 21-24

Z. Naturforsch A 26 964-672

Phys. Fluids 14 (8) 1656-1661

Phys. Fluids 18 (9) 1094-1099

Sitzungsberichte der kaiserlichen Akademie der Wissenschaften 20539

in Köhler W., Wiegand S. - Thermal

Nonequilibrium Phenomena in Fluid Mixtures, Springer, Berlin, 2002, 24-37

Phys. Rev. E 51 2013-2021

J. Chem. Phys. 100 (4) 3048-3059

J. Chem. Phys. 100 (4) 3060-3067

J. Chem. Phys. 100 (4) 3068-3078

Nature 275 (10) 565-566

Intermolecular Forces - Their Origin and

Determination, Clarendon Press, Oxford

Ann. Phys. Chem. 143 337-354 
Marrink S.-J., Berendsen H.J.C. 1994

Mason E.A., Chapman S. 1962

Maxwell J.C. 1876

Maxwell J.C. 1879

McGowan D., Evans D.J. 1986

McNab G.S., Meisen A. 1973

McQuarrie D.A. 2000

McQuarrie D.A., Simon J.D. 1997

Meyer M., Mareshal M., Hayoun M. 1988

Michels A., Wijker H., Wijker H.K. 1949

Montassier N., Boulaud D., Renoux A. 1990

Muehlner K.A., Schatz M.F., Petrov V., McCormick W.D., Swift J.B., Swinney

H.L.

Müller-Plathe F. 1997

Müller-Plathe F. 1999

Naranayan R., Schwabe D. 2003

Nepomnyashchy A.A., Velarde M.G., 2001 Colinet $\mathrm{P}$.

Nijmeijer M.J.P., Bakker A.F., Bruin C. 1988

Nishio G., Kitani S., Kazuhiro T. 1974

Nitschke K., Thess A. 1995

Nosé S. 1984

Obukhov A.M. 1941

Oertel H. jr. 2002

Ohwada T., Sone Y. 1992

Ohwada T., Sone Y., Aoki K. 1989a

Ohwada T., Sone Y., Aoki K. 1989b

Okumura H., Heyes D.M 2004
J. Phys. Chem. 98 4155-4168

J. Chem. Phys. 36 (3) 627-632

Capillary Action in Encyclopaedia Britannica 9th ed. 1876 Reprint in The Scientific Papers of James Clerk Maxwell vol 2, Dover, New York, 541-591

Philos. T. R. Soc. Lond. 170 231-256

Phys. Rev. A, 34 2133-2142

J. Coll. Interf. Sci. 44 (2)

Statistical Mechanics, University Science Books, Sausalito

Physical Chemistry, University Science Books, Sausalito

J. Chem. Phys. 89 (2) 1067-1073

Physica 15 (7) 627-633

in Masuda S., Takahashi K. - Proceedings of the 3rd International Aerosol Conference Sep 24-27 1990 Kyoto Japan, Pergamon, Oxford, 1990, 395-398

Phys. Fluids 9 (6) 1850-1852

J. Chem. Phys. 106 (14) 6082-6085

Phys. Rev. E 59 4894-4898

Interfacial Fluid Dynamics and Transport Processes, Springer, Berlin

Interfacial Phenomena and Convection, Chapman and Hall/CRC, Boca Raton

J. Chem. Phys. 89 (6) 3789-3792

Ind. Eng. Chem. Proc. D. D. 13 (4) 408-415

Phys. Rev. E 52 (6) R5772-R5775

Mol. Phys. 52 (2) 255-268

Dokl. Akad. Nauk SSSR 32 22-24

in Oertel H. jr., Prandtl - Führer durch die Strömungslehre, Friedr. Vieweg \& Sohn, Braunschweig, 329-398

Eur. J. Mech. B - Fluid 4 389-414

Phys. Fluids A 1 (9) 1588-1599

Phys. Fluids A 1 (12) 2042-2049, Erratum:

Phys. Fluids A 2 (4) 1990639

Phys. Rev. E 70061206 
Okumura H., Ito N. 2003 Phys. Rev. E 67045301

Onishi Y. 1972 T. Jpn. Soc. Aeronaut. S. 15 (29) 117-123

Ono S, Kondo S. 1960 Molecular Theory of Surface Tension in Liquids in Flügge S. (Ed.) Encyclopedia of Physics Vol 10 - Structure of Liquids, Springer, Berlin, 1960, 134-277

Onsager L. 1931a Phys. Rev. 37 (4) 405-426

Onsager L. 1931b Phys. Rev. 38 (12) 2265-2279

Ostrach S. $1982 \quad$ Annu. Rev. Fluid Mech. 14 313-345

Paolini G.V., Ciccotti G. 1987 Phys. Rev. A 35 5156-5166

Pearson J.R.A. 1958 J. Fluid Mech. 4 (5) 489-500

Perronace A., Ciccotti G., Leroy F., 2002 Phys. Rev. E 66031201 Fuchs A.H., Rousseau B.

Pertler M., Häberl M., Rommel W., 1995 Blass E.

Petrov M., Hanning A., Muehler K.A., 1998 VanHook S.J., Swinney H.L.

Petrov V., Schatz M.F., Muehlner K.A., 1996 VanHook S.J., McCormick W.D.

Piechór K. 2008 Platten J.K., Villers D. 1990

Poddoskin A.B., Yushkanov A.A., 1982 Yalamov Y.L.

Pohorille A., Cieplak P., Wilson M.A. 1996

Poincaré H.J. 1890

Poisson S.D. 1831

Pope S.B. 2003

Pratt L.R., Haan S.W. 1981a

Pratt L.R., Haan S.W. 1981b

Preisser F., Schwabe D., Scharmann A. 1983

Prigogine I. 1961

Prigogine I. 1966

Pringsheim E. 1883

Probstein R.F. 1994
Chem. Eng. Process. 34 (3) 269-277

Phys. Rev. E 58 (1) 427-433

Phys. Rev. Lett. 77 (18) 3779-3782

Arch. Mech. 60 (1) 23-58

in De Coninck J., Dunlop F., Lecture Notes in Physics vol 354 Wetting Phenomena, Springer, Berlin, 69-77

Zhurnal tekhnicheskoi fiziki 52 2253-2261, Soviet Physics - Technical Physics 27 (11) 1982 1383-1389

Chem. Phys. 204 337-345

Acta Math. 13 1-270

Nouvelle Théorie de l'Action Capillaire, Bachelier, Paris

Turbulent Flows, Cambridge University Press, Cambridge

J. Chem. Phys. 74 1864-1872

J. Chem. Phys. 74 1873-1876

J. Fluid Mech. 126 545-567

Introduction to Thermodynamics of Irreversible Processes 2nd rev ed, Interscience, New York

Non-Equilibrium Statistical Mechanics, John Wiley \& Sons, New York

Ann. Phys. 254 (1) 1-32

Physicochemical Hydrodynamics 2nd. ed., John Wiley \& Sons, New York 
Ramirez R., Risso D., Cordero P. 2000

Rao M., Berne B.J. 1979

Rapaport D.C. 1991

Rapaport D.C. 2004

Rapaport D.C. 2006

Rastogi R.P., Blokhra R.L., Agarwal 1964

R.K.

Ratogi R.P., Singh K., Singh H.P. 1969

Reid G.D., Wynne K. 2003

Reith D., Müller-Plathe F. 2000

Reynolds O. 1879

Richard B.S., Richard T.J. 1989

Richardson L.F. 1922

Riley R.J., Neitzel G.P. 1998

Rosenblatt P., LaMer V.K. 1946

Rosjorde A., Fossmo D.W., Bedeaux 2000

D., Kjelstrup S., Hafskjold B.

Rosjorde A., Kjelstrup S., Bedeaux D., 2001 Hafskjold B.

Rowlinson J.S. 1979

Rowlinson J.S., Swinton F.L. 1982

Rowlinson J.S., Widom B. 2002

Sadus R.J. 1999

Sarman S.S., Evans D.J., Cummings 1998 P.T.

Sawistowski H. 1971

Schadt C.F., Cadle R.D. 1961

Schatz M.F., Neitzel G.P. 2001

Schmipf M.E., Giddings J.C. 1987

Schmipf M.E., Giddings J.C. 1989
Phys. Rev. Lett. 85 (6) 1230-1233

Mol. Phys. 37 (2) 455-461

Phys. Rev. A 43 (12) 7046-7048

The Art of Molecular Dynamics Simulation, Cambridge University Press 2nd ed., Cambridge

Phys. Rev. E, 73 025301(R)

Transactions of the Faraday Society 60 13861390

J. Phys. Chem. 73 (9) 2798-2803

Time-Resolved Spectroscopy in Webb C.E., Jones J.D.C., Handbook of Laser Technology and Applications volIII Applications, Institute of Physics, Bristol, 2003, 2507-2528

J. Chem. Phys. 112 2436-2443

Philos. T. R. Soc. Lond. (1776-1886) $170727-$ 845

J. Phys. Chem. Ref. Data 18 (2)

Weather Prediction by Numerical Process, Cambridge University Press, Cambridge

J. Fluid Mech. 359 143-164

Phys. Rev. 70 (5/6) 385-395

J. Coll. Interf. Sci. 232 178-185

J. Coll. Interf. Sci. 240 355-364

J. Stat. Phys. 20 (2) 197-244

Liquids and Liquid Mixtures, Butterworth Scientific, London

Molecular Theory of Capillarity, Dover Publications, Mineola

Molecular Simulation of Fluids, Elsevier, Amsterdam

Phys. Rep. 305 1-92

in Hanson C., Recent Advances in LiquidLiquid Extractions, Pergamon Press, New York, 293-366

J. Phys. Chem. 65 (10) 1689-1694

Annu. Rev. Fluid Mech. 33 93-127

Macromolecules 20 1561-1563

J. Polym. Sci. B: Polymer Physics 27 1317-1332 
Schneider J, Schwabe D., Scharmann A. 1996

Schofield P., Henderson J.R. 1982

Schuster A. 1880

Schwabe D. 1981

Schwabe D. 1999a

Schwabe D., Benz S. 2002

Schwabe D., Cramer A., Schneider J., Benz S., Metzger J.,

Schwabe D., Frank S.

Schwabe D., Lamprecht R., Scharmann 1986

A.

Schwabe D., Lamprecht R., Scharmann 1988

A.

Schwabe D., Metzger J. 1989

Schwabe D., Möller U., Schneider J., 1992 Scharmann A.

Schwabe D., Scharmann A., Preisser F. 1982

Schwabe D., Tanaka S., Mizev A., 2006 Kawamura $\mathrm{H}$.

Schwabe D., Velten R., Scharmann A. 1990

Schweighofer K., Benjamin I. 1999

Scott R. L., Konynenburg van P.H. 1970

Scott W., Müller-Plathe F., van 1994 Gunsteren W.F.

Scriven L.E. 1960a

Scriven L.E. 1964

Scriven L.E., Sternling C.V. 1960b

Semenov S., Schimpf M. 2004

Sherwood T.K., Pigford R.L., Wilke 1975

C.R.

Shevtsova V.M., Mojahed M., Legros 1999 J.C.

Shiundu P.M., Williams P.S., Giddings 2003

J.C.

Shu J.Z., Yao Y.L., Hu W.R. 1994

Simic-Stefani S., Kawaji M., Yoda S. 2006

Singh B., Byers R.L. 1972
Microgravity Sci. Tech. 9 (2) 86-94

Proc. R. Soc. Lon. Ser. A 379 (1) 231-246

Philos. T. R. Soc. Lond. (1776-1886) 166 715724

Physicochem. Hydrodyn. 2 (4) 263-280

Adv. Space Res. 24 1347-1356

Adv. Space Res. 29 629-638

Adv. Space Res. 24 (10) 1367-1373

Adv. Space Res. 24 1391-1396

Naturwissenschaften 73 350-351

in Velarde M.G., Physicochemical Hydrodynamics - Interfacial Phenomena, NATO ASI Series, Series B: Physics 174, Plenum Press, New York, 1988, 291-310

J. Cryst. Growth 23-33

Phys. Fluids A 4 (11) 2368-2381

Acta Astronaut. 9 (3) 183-186

Microgravity Sci. Tech. 18 (3/4) 117-127

J. Cryst. Growth 1258-1264

J. Phys. Chem. A 103 10274-10279

Discuss. Faraday Soc. 49 87-97

Mol. Phys. 82 (5) 1049-1062

Chem. Eng. Sci. 12 98-108

J. Fluid Mech. 19 (3) 321-340

Nature 187 186-188

Phys. Rev. E 69011201

Mass Transfer, McGraw-Hill, New York

Acta Astronaut. 44 (7-12) 625-634

J. Coll. Interf. Sci. 266 366-376

Microgravity Sci. Tech. 7 (2) 83-89

Int. J. Heat Mass Tran. 49 3167-3179

Ind. Eng. Chem. Fund. 11 (1) 127-133 
Skakun S.G., Suetin P.E., Chernyak 1974 Fluid Dyn. 9 (4) 659-662

V.G.

Slattery J.C., Sagis L. Oh E.S. 2006 Interfacial Transport Phenomena 2nd ed., Springer, New York

Smith W. 2007a A Library of Software on Molecular Dynamics, Monte Carlo, Lattice Statistics and Lattice Dynamics, www.ccp5.ac.uk/librar.shtml, 03.07.2007 14:00

Smith W. 2007b The DL_POLY Molecular Simulation Package, www.cse.scitech.ac.uk/ccg/software/DL_POLY/ index.shtml, 22.06.2007 15:00

Sone Y. 1966 J. Phys. Soc. Jpn. 21 1836-1837

Sone Y. 1968b AIP Conf. Proc. 6 243-253

Sone Y. 1972 Phys. Fluids 15 (8) 1418-1423

Sone Y. 1991 Phys. Fluids A 3 (5) 1991

Sone Y. 2007 Molecular Gas Dynamics, Birkhäuser, Boston

Sone Y., Aoki K. 1977 Phys. Fluids 20 (4) 571-576

Sone Y., Sawada K., Hirano H. 1994 Eur. J. Mech. B - Fluid 13 (3) 299-303

Sone Y., Yamamoto K. 1968a Phys. Fluids 11 (8) 1672-1678

Sorensen J.M., Arlt W. 1979 in Behrensen D., Eckermann E., DECHEMA Chemistry Data Series, vol 5 Liquid-Liquid Equilibrium Data Collection, pt 1 Binary Systems, Frankfurt, 1979

Soret C. 1879 Archives des Sciences Physiques et Naturelles Periode 3 Tome 2 48-61

Soret C. 1881 Ann. Chim. Phys. Series 5 Tome 22 293-297

Spångberg D., Hermansson, K. 2003 J. Chem. Phys. 119 (14) 7263-7282

Springer G.S. 1970

J. Coll. Interf. Sci. 34 (2) 215-220

Spurk J.H. 1996 Strömungslehre 4th rev. ed., Springer, Berlin

Stecki J., Toxvaerd S. 1995

J. Chem. Phys. 103 4352-4359

Steele W.A. 1974

Interaction of Gases with Solid Surfaces, Pergamon, Oxford

Steinchen-Sanfeld 1988 in Velarde M.G., Physicochemical Hydrodynamics - Interfacial Phenomena, NATO ASI Series, Series B: Physics 174, Plenum Press, New York, 1988, 387-400

Sternling C.V., Scriven L.E. 1959 AIChE J. 5 514-523

Sternling C.V., Scriven L.E. 1964

J. Fluid. Mech. 19 321-340

Stewart R.B., Jacobsen R.T. 1989

J. Phys. Chem. Ref. Data 18 (2) 639-798

Stone A.J. 2002 The Theory of Intermolecular Forces, Clarendon Press, Oxford 
Straatsma T.P., Berendsen H.J.C., Stam 1986

A.J.

Stratmann F., Fissan H. 1989

Stuke B. 1961

Sumner L.B.S., Neitzel G.P., Fontaine 2001 J.-P., Dell'Aversana P.

Swope W.C., Andersen H.C., Berens P. 1982 H., Wilson K.R.

Takata S., Sone Y. 1995

Tegeler Ch., Span R., Wagner W. 1999

Tenenbaum A., Ciccotti G., Gallico R. 1982

Thomson J. 1855

Todd B.D., Evans D.J., Davies P.J. 1995

Tokaruk W.A., Molteno T.C.A., Morris 2000

S.W.

Toxvaerd S. 1992

Toxvaerd S., Stecki J. 1995

Trozzi C., Ciccotti G. 1984

Unbehauen H. 2002

van der Waals J.D. 1893

van der Waals J.D. 1873

van Konynenburg P.H. 1980

VanHook S.J., Schatz M.F., 1995

McCormick W.D., Swift J.B., Swinney

H.L.

VanHook S.J., Schatz M.F., Swift J.B., 1997 McCormick W.D., Swinney H.L.

Varnik F., Baschnagel J., Binder K. 2000

Vasserman A.A., Rabinovich V.A. 1967

Velarde M.G., Zeytounian R. K. 2001

Velten R., Schwabe D., Scharmann A. 1991

Villers D., Platten J.K. 1988

Villers D., Platten J.K. 1992

Vogelsang R., Hoheisel C., Paolini 1987 G.V., Ciccotti G.
Mol. Phys. 57 (1) 89-95

J. Aerosol Sci. 20 (8) 899-902

Chem. Eng. Tech. 33 173-181

Phys. Fluids 13 (1) 107-120

J. Chem. Phys. 76 (1) 637-649

Eur. J. Mech. B - Fluid 14 487-518

J. Phys. Chem. Ref. Data 28 (3)

Phys. Rev. A 25 (5) 2778-2787

The London and Edinburgh Philosophical

Magazine and Journal of Science 10 330-333

Phys. Rev. E 52 1627-1638

Phys. Rev. Lett. 84 3590-3593

Mol. Phys. 76 (6) 1397-1409

J. Chem. Phys. 102 (18) 7163-7168

Phys. Rev. A 29 (2) 916-925

Regelungstechnik 1st vol 12th ed, Friedrich Vieweg und Sohn, Braunschweig

Verhandelingen van de Koninklijke Akademie van Wetenschappen te Amsterdam Sectie 1 Deel 1 (8) $1-56$

Over de Continuiteit van den gasen vloeistoftoestand, Sijthoff, Leiden

Philos. T. R. Soc. Lond. Ser. A 298 495-540

Phys. Rev. Lett. 75 (24) 4397-4400

J. Fluid Mech. 345 45-78

J. Chem. Phys. 113 (10) 4444-4453

Inzhenero-Fizicheskii Zhurnal 13 (2) 190-198

Interfacial Phenomena and the Marangoni Effect, Springer, Wien

Phys. Fluids A 3 (2) 267-279

Appli. Sci. Res. 45 145-152

J. Fluid Mech. 234 487-510

Phys. Rev. A 36 3964-3974 
Volkov A.G., Deamer D.W., Tanelian 1998 D.L., Markin V.S.

Waldmann L. 1966

Waldmann L. 1967

Walton J.P.R.B., Tildesley D.J., 1983 Rowlinson J.S.

Weber E.H. 1854

Wiegand S. 2004

Wiegand S. 2006

Wittko G.A. 2007

Wold I., Hafskjold B. 1999

Xu J., Kjelstrup S., Bedeaux D., 2006 Rosjorde A., Rekvig L.

Xue L., Keblinski P., Phillpot S.R., 2004 Choi S.U., Eastman J.A.

Yalamov Y.I., Ivchenko I.N., Deryagin 1968 B.V.

Yao Y.L., Shu J.Z., Xie J.C., Liu F., Hu 1997 W.R.

Yoshizawa M., Kurosawa M. 1999 Young T. 1805

Younglove B.A., Hanley H.J.M. 1986

Zhou B., Liu Q., Hu L., Yao Y., Hu W. 2002
Liquid Interfaces in Chemistry and Biology, John Wiley \& Sons New York

in Davies C.N., Aerosol Science, Academic Press, London, 1966, 137-162

Z. Naturforsch. A 22 1269-1280

Mol .Phys. 48 (6) 1357-1368

Berichte über die Verhandlungen der königlich sächsischen Gesellschaft der Wissenschaften; Mathematisch-Physische Classe 1854 57-67

J. Phys.-Condens. Matt. 16 R357-R379

Bunsen-Magazin 8 130-134

Über den Einfluß molekularer Parameter auf die Transporteigenschaften organischer Lösungsmittel, Dissertation, Bayreuth

Int. J. Thermophys. 20 847-856

J. Coll. Interf. Sci. 299 452-463

Int. J. Heat Mass Trans. 47 4277-4284

Doklady Akademii Nauk SSSR 175 (3) 549-552 [Soviet Physics Doklady 12 (7) 1968 714-716]

Int. J. Heat Mass Trans. 40 (11) 2517-2524

Phys. Rev. A 61013808

Philos. T. R. Soc. Lond. 95 65-87

J. Chem. Phys. 15 (4) 1323-1337

Sci. Chin. E 45 (5) 552-560 


\section{Appendix A: Fluctuations in Selected Observables}

We determined, in addition to many observables themselves, also their fluctuations (standard deviations). They turned out to be quite useful for our purposes in various ways. Thus, comparing the fluctuations with their physical estimates serves as another means to assess the approximation of reality by our systems (see chapter 5.1). Although we did not determine the material properties in the estimation formulas, we could at least use them to double-check the qualitative dependencies on the other observables. Particularly the velocity fluctuations helped us later the other way round, when estimating the properties of the viscosities (see section 5.2.2). Landau and Lifshitz derive in detail expressions for the fluctuations in selected observables [landau2007]. The authors use statistical physics (see part 9) as a starting point. They focus on a small but still macroscopic section of a given system. Their derivations hold if it is in or, at least approximately, if it is only moderately out of equilibrium. In larger sections, however, the fluctuations become more and more subject to the external constraints.

We shall compile the most important expressions here. Thus, the temperature fluctuations, $\sigma(T)$, are the temperature, $T$, multiplied by the square root of Boltzmann's constant, $k_{B}$, over the extensive heat capacity, $C_{v}$, at constant volume [landau2007]:

$$
\sigma(T)=T \sqrt{\frac{k_{B}}{C_{v}}} .
$$

We skipped its computation in our simulations, since it requires the knowledge of the difficult to determine temperature dependence of the local internal energies. We could rather estimate the main dependencies of the heat capacities from their statistical mechanical definition [croxton1975]:

$C_{v}=\frac{3}{2} N k_{B}+\frac{4 \pi \rho^{2}}{2} \int_{0}^{\infty}\left(\frac{\partial \rho_{i j}\left(r_{i j}\right)}{\partial T}\right)_{V} u_{i j}\left(r_{i j}\right) r_{i j}^{2} d r_{i j}$.

Here, $N$ represents the particle number in the considered section and $\rho$ its density. $u_{i j}\left(r_{i j}\right)$ is the interatomic pair potential and $\rho_{i j}\left(r_{i j}\right)$ the pair distribution as function of the particle distance, $r$. Equation (8-2) indicates generally an increase of the heat capacity with increasing particle number.

We shall look, in addition to the temperature fluctuations, at those of the density. They are the overall density, $\rho$, multiplied by the square root of Boltzmann's constant, $k_{B}$, the temperature, $T$, and 
the isothermal compressibility factor, $\kappa_{T}$, over the volume, $V$, of the considered section [hansen1969], [croxton1975], [luo1995], [hadjiconstantinou2003], [landau2007]:

$\sigma(\rho)=\rho \sqrt{\frac{k_{B} T \kappa_{T}}{V}}$

The isothermal compressibility factor is, similar to the heat capacity above, difficult to compute in our simulations. Moreover, it depends generally only little on the composition and the state of a liquid, anyhow [rowlinson1982]. The same should hold above all for the simple liquids considered here. Hence, we can disregard the exact value of the compressibility factor for our purposes.

Finally, the com velocity fluctuations are of particular interest to us. Computing them requires, different from the other ones above, no additional material properties. Hence, we could verify the validity of this expression by Landau and Lifshitz quantitatively in our simulations. According to it, the velocity fluctuations have a standard deviation, $\sigma(v)$, equal to Boltzmann's constant, $k_{B}$, times the temperature, $T$, divided by the total mass, $M$, in the considered section [landau2007]:

$$
\sigma(v)=\sqrt{\frac{k_{B} T}{M}}
$$

Since they are isotropic, we omit the subscript of the velocity component considered, i.e. $\sigma(v)=\sigma\left(v_{x}\right)=\sigma\left(v_{y}\right)=\sigma\left(v_{z}\right)$.

For the sake of completeness, we would like to comment on the fluctuations of any extensive mechanical observable. They generally increase in comparison to its value the smaller the considered section is. Chapter 3.1 mentions this dependence already. It is the reason why many observables loose their macroscopic meaning on arbitrarily small length scales. One can show by relatively simple considerations that the ratio between the standard deviation, $\sigma(o)$ of an observable, $o$, and its value, $\mu(o)$, increases inversely proportional to the square root of the particle number, $N$, in the considered section [haile1997]:

$$
\frac{\sigma(o)}{\mu(o)} \propto \frac{1}{\sqrt{N}}
$$




\section{Appendix B: Basic Assumptions of Statistical Physics}

Traditional statistical physics acknowledges the fact that the exact phase space trajectory of a multiparticle system (masses interacting with each other through arbitrary potentials) cannot be analytically computed [poincare1890]. Furthermore, Boltzmann's classical argument [hill1986], [hill1987], [mcquarrie2000], [landau2007] postulates that this trajectory is known for all times, i.e. infinitely. However, functions, called "partition functions", which allow computing many thermodynamic properties, can be obtained in many cases in spite of these limitations. The computation of these partition functions for systems under different external constraints (volume, energy, etc.) is the main goal of traditional (equilibrium) statistical mechanics.

Let us consider the trajectory, or a finite, representative parts of it, possibly available only at discrete consecutive times $t_{0}, t_{1}, t_{2}, \ldots$. The trajectory can be considered to consist of "points", or "microstates", each specified through the generalised particle coordinates, $\mathbf{q}$, and momenta, $\mathbf{p}$, of all particles at the given time. We can then compute ensemble averages of a certain mechanical observable, $o$, by weighting each of its individual values, $o(\mathbf{q}, \mathbf{p})$, with their probability of occurrence, $\rho(\mathbf{q}, \mathbf{p})$, and summing/integrating the product over all microstates:

$\mu(o)=\int o(\mathbf{q}, \mathbf{p}) \rho(\mathbf{q}, \mathbf{p}) \mathrm{d} \mathbf{q} \mathrm{d} \mathbf{p}$.

This average results from the so-called statistical distribution function, $\rho$. If NVE constraints (see chapter 4.1) apply, for instance, it is homogeneously distributed over all microstates, compatible with these constraints (second postulate of statistical mechanics [hill1986]). A comparison of this averaging in traditional statistical physics (which leads to the concept of the partition function) and MD simulation yields their main difference.

One now considers over brief time intervals several entities of the same system instead of attempting to compute a single long (and representative) section of the phase space trajectory. Done properly, such ensemble averages can also describe nonstationary processes, provided the external constraints of the ensemble are unambiguously defined and the deviation from equilibrium is "moderate" (see part 10). In an equilibrium system, both the ensemble and time averages deliver, according to the first postulate of statistical mechanics [hill1986], the same results. 
When dealing with nonequilibrium systems, one must know their time-dependent statistical distribution function, $\hat{\rho}(\mathbf{q}, \mathbf{p}, t)$. Its evolution is described by the Liouville equation [prigogine1966]:

$$
\frac{\partial \hat{\rho}}{\partial t}=-\sum_{i=1}\left(\frac{\partial \hat{H}}{\partial \hat{p}_{i}} \frac{\partial \hat{\rho}}{\partial \hat{q}_{i}}-\frac{\partial \hat{H}}{\partial \hat{q}_{i}} \frac{\partial \hat{\rho}}{\partial \hat{p}_{i}}\right) .
$$

Here, $\hat{H}$ represents the Hamiltonian while the index, $i$, runs over all generalised coordinates, $\hat{p}_{i}$, and momenta, $\hat{q}_{i}$, of the system. Even if suitable initial conditions were known, a general analytical solution of the Liouville equation is as impossible as is such a solution for the particle equations of motion underlying the trajectory. Only very special cases, e.g. in perturbation theory which requires stringent simplifications essentially concerning the interparticle interactions, allow for such a solution. However, if it is possible, the macroscopic system behaviour would result from ensemble averages over the time-dependent statistical distribution function. This is the leading idea of the present work. 


\section{Appendix C: Basic Assumptions of Irreversible Thermodynamics}

Irreversible thermodynamic processes are said to proceed "moderately", if they can be described by linear irreversible thermodynamics [haase1963]. It can be shown that this theory is compatible with the following assumptions [degroot1960]:

- First, the system entropy production is positive.

- Second, during irreversible processes small but macroscopic subsystems of the system can be described by intensive local thermodynamic state variables at any time, so that the second law of thermodynamics and the Gibbs Equation can be formulated using these variables.

- Third, based on the definition of local thermodynamic state variables, forces (e.g. gradients of pressure, temperature, or chemical potential) drive fluxes of e.g. momentum, heat, or mass, in nonequilibrium systems. The fluxes are linearly coupled to the forces via phenomenological coefficients.

- Finally Onsager's reciprocity relations are fulfilled, i.e. the matrix of the phenomenological coefficients is symmetric.

Stationary nonequilibrium systems have been investigated in NEMD simulations, mainly to study shear flows and heat conduction. The simulation results agree quite well with the system behaviour to expect from irreversible thermodynamics if the latter is applied on the scale of the simulation. Even the assumptions of irreversible thermodynamics could be shown to be fulfilled in NEMD simulations [rosjorde2000], [hafskjold1995], [tenenbaum1982], [ciccotti1980], [ikeshoji1994], [mcgowan1986], [paolini1987]. Particularly in boundary driven (BD) ones, such a strong approximation of reality applies, of course, only in the investigation regions. We mentioned already in section 3.2.2 how the error form the strongly simplified implementation of the boundaries slowly abates remote from them. 


\section{Appendix D: Phase Behaviour of the Ar5Ar5 Mixture}

According to thermodynamics, typically several properties specify unambiguously the entire phase behaviour of a given substance or mixture. When dealing with them in general, one states intensive properties, i.e. ones that are independent of the considered amount of matter. The mandatory number of them, which is required for such a description, results from Gibbs's phase rule [gibbs1875], [findlay1951]:

$N_{F}=N_{C}-N_{P}+2$.

Here, the number of degrees of freedom, $N_{F}$, is the number of components, $N_{C}$, minus the number of phases present in the matter, $N_{P}$, plus two. Plots of its aggregation as function of the independent state variables are well known as phase diagrams. We show, for instance, the familiar one of water in Figure 77 . Note in it, how a pure substance $\left(N_{C}=1\right)$ has, according to the phase rule, generally $\left(N_{P}=1\right)$ two independent state variables, chosen here to be the temperature, $T$, and the pressure, $P$. Their number decreases to one at the phase transition curve $\left(N_{P}=2\right)$ or even to zero at the triple point $\left(N_{P}=3\right)$. When dealing with a specific piece of matter, however, the intensive properties alone do not suffice to specify its state unambiguously. Instead, at least one additional extensive state variable is necessary. Thus, all possible liquid-vapour equilibrium states, for instance, are typically displayed in a $P V T$-diagram with the volume, $V$, as an additional variable (see Figure 78).

We consider binary mixtures in this work. They consist of two components $\left(N_{C}=2\right)$ and have generally three degrees of freedom. The phase behaviour of such mixtures is typically displayed in PxT-diagrams. Here, $x$ represents the mole fraction of one component. Their appearance can differ strongly, depending on the mixture in consideration. Scott and van Konynenburg [scott1970] subdivided them into six main groups by disregarding the composition dependence.

We chose the interactions of our Ar5 particles so as to make them partially miscible. It was, however, unclear, at first, at which states they would separate. We estimated their phase behaviour in two ways: by a series of simulations and by application of the virial equation with a mixing rule. Both ways will be explained below. Figure 79 shows the phase diagram obtained from the MD simulations. It is for an equimolar composition. Two lines confine the region of partial liquid miscibility to the right, a line of liquid-liquid-vapour triple points (TRPs), where evaporation starts, 
and a line of critical solution points (CSPs), where the mixture mixes entirely while becoming supercritical. Both lines intersect at the critical end point (CEP). We estimated its location $\left(P=4.96 \mathrm{MPa}, T=117 \mathrm{~K}, x_{A r(5}=0.5\right)$ at the intersection of their extrapolations. The phase behaviour described up to here is clearly of the type II in the subdivision by Scott and van Konynenburg. We thus likely come across suitable simulation parameter values that allow partial liquid miscibility throughout an entire nonequilibrium interfacial system, if we adjust them so as to establish pressures above and temperatures on the order of those at this end point.

Figure 79 shows additionally the critical solution points found by using the virial equation. They have the same qualitative trend as those obtained in the MD simulations. We must keep the different ranges of the considered particle interactions in mind when comparing the different data. The same holds also for comparisons with the data obtained in our actual studies. Thus, we determined in the system, E-Ar5Ar5-0.6-1.0-3346-3524-4.74x8.00x9.40-120, for instance, a phase composition of $x_{M a j}=0.968$. It is clearly smaller than the one of $x_{M a j}=0.999$ predicted by the virial equation at the same pressure and temperature. Finally, Kofke's [kofke1993] and Agrawal's [agrawal1995] dimensionless phase diagram describes already very thoroughly the phase behaviour

of our pure Ar-like substances. We integrated it, together with the above one of the equimolar mixture, into a qualitative PxT-diagram (see Figure 80).

\section{Phase Behaviour from EMD Simulations}

Several states of the Ar5Ar5 mixture must be simulated to learn about its phase behaviour. Hence, we designed a computationally inexpensive simulation system that we shall introduce here first. It is cubic with periodic boundaries. They contain an equimolar mixture of $4096 \operatorname{Ar} 5$ and $4096 \operatorname{Ar} 5$ particles whose interactions are cut-off beyond a distance of $r_{c}=0.9 \mathrm{~nm}$. We maintained the system temperature at a given value by using our thermostating algorithm in the same way as in other EMD simulations before (see chapter 4.1). Both the volume and the temperature were systematically varied in subsequent simulations. Each of them is two million time steps long, of which we considered only every thousandth one of the second half in the analysis. These simulations might appear oversimplified in comparison to the other ones in this work. Nevertheless, we are still within the scope of what turned out sufficient in previous semiquantitative studies from the literature. In the analysis, we determined the system pressure. It is plotted as function of the volume and temperature in Figure 81. Moreover, we determined the number and the kind of the present phases 
by comparing the densities in different subvolumes of the system. Each of them has a twenty-fifth of its volume.

We would like to add some comments on how we determined the phase transition curves in the simulations. There rests some arbitrariness in them, since the appearance of a new or the disappearance of an existing phase is hard to detect. Thus, the pressure fluctuates, for instance, as function of the volume when the liquid starts to evaporate. Such fluctuations resemble those in a cubic equation of state during evaporation. Think, for instance, of the equation by van der Waals [vanderwaals1873]. According to him, they represent metastable states. Hansen et al. confirmed this interpretation in MD simulations of an "evaporating" pure substance [hansen1969]. They artificially kept it from separating into two phases and, as a result, observed the same fluctuations. We may be dealing with a similar phenomenon in our liquid mixture. A small bubble cannot exist permanently due to the reduced vapour pressure in it. Its drop decreases as the gas phase grows, which could also explain the otherwise constant system pressure until both phases have evaporated completely. Hence, we shall assume this pressure also at the dew and bubble point of the mixture at the given temperature. To conclude, Figure 82 shows different phase arrangements found in the liquid-liquidvapour region. They may appear rather unusual at first sight due to the periodicity in the system. Nevertheless, they always behave such that the interfacial area amounts to a minimum, which is in agreement with thermodynamic considerations.

There is also an unambiguous thermodynamic definition of the critical solution points. The chemical potentials used by it are, however, difficult to determine in MD simulations. Instead, we estimated the disappearance of the partial miscibility only from the convergence of the local densities in the system. Their natural fluctuations and finite size effects are simply disregarded. The densities tend to distribute more evenly as the system approaches the critical solution point. We thus assumed it, the first time their extreme values differ again more along an isotherm.

\section{Phase Behaviour from the Virial Equation of State}

We estimated the critical solution points of our mixture by using the virial equation of state. Strictly speaking, it is an infinite series,

$Z=\frac{P v}{R T}=1+\frac{A_{2}}{v}+\frac{A_{3}}{v^{2}}+\ldots$ 
where the compressibility factor, $\mathrm{Z}$, is the pressure, $P$, times the molar volume, $v$, divided by the universal gas constant, $R$, and the temperature, $T$. The symbols, $A_{2}, A_{3}, \ldots$, are called virial coefficients. Each of them describes the particle interactions of another order which is additionally naming. Thus, the third virial coefficient, $A_{3}$, for instance, accounts for the three particle interactions. In gases of simple molecules, there is generally a fair representation of the compressibility factor to nearly the critical density, if the second and third virial coefficients are known. We can, in principle, compute their values from the interactions between the particles, $i$ and $j$ :

$$
\begin{aligned}
& A_{2}=2 \pi N_{A} \int_{0}^{\infty}\left[1-e^{-u_{i j}(r) / k_{B} T}\right] r_{i j}^{2} \mathrm{~d} r_{i j} \\
& A_{3}=\frac{-8 \pi^{2} N_{A}^{2}}{3} \int_{0}^{\infty} \int_{0}^{\infty} \int_{\left|r_{12}-r_{13}\right|}^{r_{12}+r_{13}} f_{12} f_{13} f_{23} r_{12} r_{13} r_{23} \mathrm{~d} r_{12} \mathrm{~d} r_{13} \mathrm{~d} r_{23}, \text { where } f_{i j}=e^{-u_{i j}\left(r_{i j}\right) / k_{B} T}-1
\end{aligned}
$$

The subscripts run over all particles in the three-body interaction. Their distances are symbolised as $r_{i j}$ and enter into the applicable pair potential function $u_{i j}\left(r_{i j}\right)$. The symbols, $N_{A}$ and $k_{B}$, represent Avogadro's and Boltzmann's constant. A mixing rule must be additionally applied in a mixture:

$\overline{A_{2}}=\sum_{i=1}^{N_{c}} \sum_{j=1}^{N_{c}} x_{i} x_{\breve{\jmath}} A_{\check{\jmath}}$

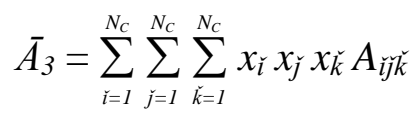

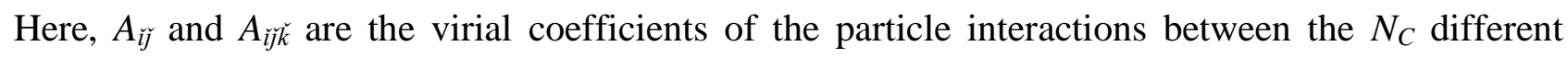
components $\check{\imath}, \breve{j}$, or $\check{k}$ in the mixture while the symbols $x_{\check{l}}, x_{\breve{j}}$, or $x_{\breve{k}}$ represent their mole fractions.

Since our simulations account only for pairwise interactions, the third and all higher virial coefficients can be neglected. We computed the remaining second coefficients for our Ar5Ar5 mixture. Table 35 lists their values as function of the temperature. They are for the equimolar composition considered here. We then computed from them the fugacity coefficients of the individual components, $\breve{\iota}$, as part of the mixture

$\ln \varphi_{\check{l}}=\left(2 \sum_{j=1}^{N_{C}} x_{\check{J}} A_{\check{l}}-\overline{A_{2}}\right) \frac{P}{R T}$

and as pure substances,

$\ln \varphi_{\check{\imath} 0}=\frac{A_{\check{~}} P}{R T}$. 
Further computations eventually delivered the activity coefficients, $\gamma_{i}$,

$\ln \gamma_{\check{i}}=\frac{\ln \varphi_{\check{l}}}{\ln \varphi_{\check{\imath} 0}}$

the activities, $a_{i}$,

$a_{\grave{\imath}}=x_{i} \gamma_{\check{i}}$

the molar excess Gibbs energy, $g^{E}$,

$g^{E}=\mathrm{RT} \sum_{i=1}^{N_{c}} x_{\grave{\imath}} \ln \gamma_{i}$

or the molar Gibbs energy change of mixing, $\Delta_{\text {mix }} g$,

$\Delta_{m i x} g=g^{E}+\mathrm{RT} \sum_{i=1}^{N_{C}} x_{i} \ln x_{\check{\imath}}$

Figure 83 shows an example of their composition dependence at a temperature of $T=180 \mathrm{~K}$. Partial liquid miscibility occurs, when the second derivative of the Gibbs energy of mixing is negative. Then, there is a "butterfly" in a plot of both activities as function of each other (see Figure 84a). We systematically varied the pressure in steps of $1 \mathrm{MPa}$ until such a butterfly appeared for the first time (see Figure 84b). The thus obtained pressure constitutes, in combination with the present temperature, an estimate of the critical solution point. We estimated further ones following the same procedure also at other temperatures. 


\section{Appendix E: 2D Liquid-Liquid Nonequilibrium Interfacial System}

We considered up to here only the macroscopic system behaviour. It involves, however, on the smaller length and time scales even much more complex fluctuations. They might as well play an important role in the thermocapillary effect. We could catch a first insight by inhibiting them in additional two-dimensional simulations of a liquid-liquid interfacial system. See Figure 85 for a snap shot of a configuration.

The two-dimensional system had in principle the same setup as the previously studied ones, when disregarding their additional $x$-dimension. Nevertheless, there lie many differences in the details of the behaviour. Thus, a new packaging renders, for instance, favourable for the particles. Several changes turned out useful in response. We increased the segregation of the mixture by omitting the attractive term in the interaction of unlike particles. Moreover, we increased the cut off radius to $1.43 \mathrm{~nm}$. Such a force field features sharp interfaces over a wide enough temperature range to detect the convection clearly. In addition to the force field, we changed the dimension of the system. It had lengths of $L_{y}=20.0 \mathrm{~nm}$ in $y$ - and of $L_{z}=46.0 \mathrm{~nm}$ in $z$-direction. A subdivision of them into $n_{y}=10$ by $n_{z}=40$ subvolumes provides a suitable spatial resolution to describe the local behaviour. Each of them contains on the average approximately 20.5 of a total of 5408 particles in the equimolar mixture. Finally, we decreased the thermostated temperatures to values of $T_{C}=60 \mathrm{~K}$ in the cold and of $T_{H}=100 \mathrm{~K}$ in the hot region. They further promote the segregation of the mixture. Both regions have a width of $1.0 \mathrm{~nm}$, which renders suitable for the thermostating. The above changes enter into a brief denomination of the system, N-Ar5Ar5-0.6r-1.0-2704-2704-20.0x46.0-60-100. A letter "r", attached to the LB mixing parameter of $\xi=0.6$, indicates the exclusively repulsive character of the cross interactions. All other aspects of the simulations and their analysis agree with those in the studies of the three dimensional systems. We adapted the simulation code used for them accordingly. The preparational runs covered 6 million (30ns) and the production ones 15 million time steps (75ns).

Figure 86 shows the spatial variation of the local observables. Their distributions resemble those found in the previous systems. Such a similarity hints at a two-dimensional character of the thermocapillary effect. Its occurrence requires at least no fluctuations in $x$-direction at all. If and to 
what extent they affect the flows remains subject to further research. It goes, however, beyond the scope of this work. We discuss on the same account only those observables that are necessary to establish the convection. The other observables determined additionally in previous simulations, e.g. the standard deviations or the particle and volume forces, exhibit no fundamentally new behaviour. 


\section{Symbols and Abbreviations}

\begin{tabular}{|c|c|c|}
\hline Latin Symbol & Unit & Description \\
\hline$A$ & $\mathrm{~J}$ & Helmholtz free energy \\
\hline$\overline{A_{2}}$ & - & 2nd virial coefficient in a mixture \\
\hline$\overline{A_{3}}$ & - & 3 nd virial coefficient in a mixture \\
\hline$a_{\check{l}}$ & - & activity of the substance $\check{l}$ \\
\hline$A_{\check{l} \check{j}}$ & - & $\begin{array}{l}\text { 2nd virial coefficient of the interaction between the } \\
\text { substances } \check{l} \text {, and } \check{j}\end{array}$ \\
\hline$A_{i j \breve{k}}$ & - & $\begin{array}{l}\text { 3rd virial coefficient of the interaction between the } \\
\text { substances } \check{l}, \breve{J} \text {, and } \check{k}\end{array}$ \\
\hline$a_{t}$ & - & thermal accommodation coefficient \\
\hline$C$ & $\mathrm{~m} / \mathrm{s}$ & spatial velocity correlation function \\
\hline$c_{P}$ & $\mathrm{~J} /(\mathrm{kg} \cdot \mathrm{K})$ & heat capacity at constant pressure \\
\hline $\mathrm{C}_{\mathrm{v}}$ & $\mathrm{J} / \mathrm{K}$ & extensive heat capacity at constant volume \\
\hline$e$ & $\mathrm{~J} / \mathrm{m}^{3}$ & local internal energy \\
\hline E & $\mathrm{J}$ & internal system energy \\
\hline $\mathbf{e}_{i^{\prime}}$ or $\mathbf{e}_{j}$ & $\mathrm{~m}$ & unit vector in $i^{\prime}$ - or $j^{\prime}$ - direction \\
\hline $\mathbf{f} / f_{i^{\prime}}$ & $\mathrm{N} / \mathrm{m}^{3}$ & volume force / in $i^{\prime}$-direction \\
\hline $\mathbf{f}^{\mathrm{ext}}$ & $\mathrm{N} / \mathrm{m}^{3}$ & external volume force vector \\
\hline $\mathbf{f}^{\mathrm{int}}$ & $\mathrm{N} / \mathrm{m}^{3}$ & internal volume force \\
\hline$f$ & $\mathrm{~s}^{3} / \mathrm{m}^{3}$ & particle velocity distribution function \\
\hline$f_{0}$ & $\mathrm{~s}^{3} / \mathrm{m}^{3}$ & MB particle velocity distribution function \\
\hline$f_{1}$ & $\mathrm{~s}^{3} / \mathrm{m}^{3}$ & $\begin{array}{l}\text { bias of the MB particle velocity distribution function in a } \\
\text { temperature gradient }\end{array}$ \\
\hline$f_{\text {inc }}$ & $\mathrm{s}^{3} / \mathrm{m}^{3}$ & $\begin{array}{l}\text { velocity distribution function of the particles incident on } \\
\text { a wall }\end{array}$ \\
\hline$f_{\text {rep }}$ & $\mathrm{s}^{3} / \mathrm{m}^{3}$ & $\begin{array}{l}\text { velocity distribution function of the particles reflected } \\
\text { from a wall }\end{array}$ \\
\hline$G$ & $\mathrm{~J}$ & Gibbs free energy \\
\hline$g^{\mathrm{E}}$ & $\mathrm{J} / \mathrm{mol}$ & molar excess Gibbs energy \\
\hline$\Delta_{\text {mix }} \mathrm{g}$ & $\mathrm{J} / \mathrm{mol}$ & molar Gibbs energy change of mixing \\
\hline$H$ & $\mathrm{~J}$ & enthalpy \\
\hline$\hat{H}$ & $\mathrm{~J}$ & Hamiltonian \\
\hline $\mathbf{i} / i_{i^{\prime}}$ & $\mathrm{N} / \mathrm{m}^{3}$ & interatomic volume force / in $i^{\prime}$-direction \\
\hline $\mathbf{I}(\mathbf{r}) / I_{i^{\prime} j^{\prime}}$ & $\mathrm{N} / \mathrm{m}^{2}$ & interatomic contribution to the stress tensor / element $i^{\prime} j^{\prime}$ \\
\hline
\end{tabular}




\begin{tabular}{|c|c|c|}
\hline Latin Symbol & Unit & Description \\
\hline $\mathbf{k} / k_{i^{\prime}}$ & $\mathrm{N} / \mathrm{m}^{3}$ & kinetic volume force / in $i^{\prime}$-direction \\
\hline $\mathbf{K}(\mathbf{r}) / K_{i^{\prime} j^{\prime}}$ & $\mathrm{N} / \mathrm{m}^{2}$ & kinetic contribution to the stress tensor / element $i^{\prime} j^{\prime}$ \\
\hline$k_{B}$ & $\mathrm{~J} / \mathrm{K}$ & Boltzmann's constant \\
\hline$k_{D}$ & - & differential gain of a thermostat \\
\hline$k_{I}$ & - & integral gain of a thermostat \\
\hline$k_{P}$ & - & proportional gain of a thermostat \\
\hline$K_{x}$ & $\mathrm{~J}$ & kinetic energy of the particle $x$-movement \\
\hline$\hat{K}_{x}$ & $\mathrm{~J}$ & $\begin{array}{l}\text { kinetic energy of the particle } x \text {-movement after the } \\
\text { thermostating }\end{array}$ \\
\hline$\Delta K_{x}$ & $\mathrm{~J}$ & change in the kinetic energy of the particle $x$-movement \\
\hline$K n$ & - & Knudsen number \\
\hline$l$ & $\mathrm{~m}$ & characteristic length of the largest eddies \\
\hline$L$ & $\mathrm{~m}$ & characteristic length \\
\hline$L_{t}$ & $\mathrm{~s}$ & simulation length \\
\hline$L_{x}, L_{y}, L_{z}$ & $\mathrm{~m}$ & system dimension in $x$-, $y$-, $z$-direction \\
\hline$m$ & $\mathrm{~kg}$ & particle mass \\
\hline$m_{i}, m_{i}$ or $m_{j}$ & $\mathrm{~kg}$ & $\begin{array}{l}\text { particle mass of the substance } \check{l} \text {, e.g. ArA or ArB, or of } \\
\text { the particles } i \text { or } j\end{array}$ \\
\hline$M$ & $\mathrm{~kg} / \mathrm{mol}$ & molar mass \\
\hline $\mathbf{n}$ & - & unit normal vector \\
\hline$N$ & - & number of particles \\
\hline$N_{C}$ & - & number of components in a system \\
\hline$N_{F}$ & - & $\begin{array}{l}\text { number of thermodynamic degrees of freedom in a } \\
\text { system }\end{array}$ \\
\hline$N_{\check{\imath}}$ & - & particle number of the substance $\check{\imath}$, e.g. ArA or ArB \\
\hline$N_{P}$ & - & number of phases in a system \\
\hline$o$ & - & arbitrary observable \\
\hline$\hat{p}_{i}$ & & $\begin{array}{l}\text { momentum along the generalised coordinate } i \text { of a } \\
\text { system }\end{array}$ \\
\hline $\mathbf{p}$ & $\mathrm{m} / \mathrm{s}$ & vector of all particle momenta \\
\hline $\mathbf{p}_{i}$ or $\mathbf{p}_{j}$ & $\mathrm{~kg} \cdot \mathrm{m} / \mathrm{s}$ & linear momentum of the particle $i$ or $j$ \\
\hline$P$ & $\mathrm{~N} / \mathrm{m}^{2}$ & pressure \\
\hline$P e$ & - & Péclet number \\
\hline$\hat{q}_{i}$ & & location along the generalised coordinate $i$ of a system \\
\hline$\delta \dot{q}$ & $\mathrm{~W} / \mathrm{m}^{3}$ & heat flux into a system \\
\hline$\dot{Q}$ & $\mathrm{~W}$ & heat transfer through a system \\
\hline$\dot{Q}_{C}$ & $\mathrm{~W}$ & heat removal from the cold thermostated region \\
\hline
\end{tabular}




\section{Latin Symbol Unit Description}

\begin{tabular}{|c|c|c|}
\hline$\dot{Q}_{H}$ & $\mathrm{~W}$ & heat supply to the hot thermostated region \\
\hline $\mathbf{q}$ & $\mathrm{m}$ & vector of all particle locations \\
\hline$r$ & $\mathrm{~m}$ & particle distance \\
\hline$r_{c}$ & $\mathrm{~m}$ & cut-off radius \\
\hline$r_{i j}$ & $\mathrm{~m}$ & $\begin{array}{l}\text { length of the connecting vector between the particle } j \\
\text { and } i\end{array}$ \\
\hline$R$ & $\mathrm{~J} /(\mathrm{K} \cdot \mathrm{mol})$ & universal gas constant \\
\hline $\operatorname{Re}$ & - & Reynolds number \\
\hline $\mathbf{r}$ & $\mathrm{m}$ & location vector \\
\hline $\mathbf{r}_{i}$ or $\mathbf{r}_{j}$ & $\mathrm{~m}$ & location vector of particle $i$ or $j$ \\
\hline $\mathbf{r}_{i j}$ & $\mathrm{~m}$ & connecting vector between the particles $i$ and $j$ \\
\hline$s$ & $\mathrm{~J} /\left(\mathrm{K} \cdot \mathrm{m}^{3}\right)$ & local entropy \\
\hline$s_{f f 1}$ & $\mathrm{~N} / \mathrm{m}$ & slope with which the external force ff 1 increases \\
\hline$S$ & $\mathrm{~J} / \mathrm{K}$ & entropy \\
\hline$\delta S$ & $\mathrm{~m}^{2}$ & macroscopically small surface element \\
\hline$\Delta S$ & $\mathrm{~m}^{2}$ & macroscopic surface element \\
\hline$\dot{S}_{g e n}$ & $\mathrm{~W} / \mathrm{K}$ & entropy production in a system \\
\hline$S_{i^{\prime}=j^{\prime}}$ & $\mathrm{N} / \mathrm{m}^{2}$ & normal stresses \\
\hline$S_{i^{\prime} \neq j^{\prime}}$ & $\mathrm{N} / \mathrm{m}^{2}$ & shear stresses \\
\hline $\mathrm{S}_{\mathrm{iijj}}$ & $\mathrm{N} / \mathrm{m}^{2}$ & arithmetic average of the normal stresses $S_{i^{\prime} i^{\prime}}$ and $S_{j^{\prime} j^{\prime}}$ \\
\hline$\delta \dot{S}_{\text {irrev }}$ & $\mathrm{W} /\left(\mathrm{K} \cdot \mathrm{m}^{3}\right)$ & entropy production \\
\hline$\delta S_{j^{\prime}}$ & $\mathrm{m}^{2}$ & $\begin{array}{l}\text { macroscopically small cut surface perpendicular to the } j^{\prime}- \\
\text { axis }\end{array}$ \\
\hline$\delta \dot{S}_{r e v}$ & $\mathrm{~W} /\left(\mathrm{K} \cdot \mathrm{m}^{3}\right)$ & entropy flux \\
\hline$\dot{S}_{C}$ & $\mathrm{~W} / \mathrm{K}$ & entropy flux out of the cold thermostated region \\
\hline$\dot{S}_{H}$ & $\mathrm{~W} / \mathrm{K}$ & entropy flux into the hot thermostated region \\
\hline$S_{T}$ & $\mathrm{~K}^{-1}$ & Soret coefficient \\
\hline$S_{T, \check{\imath}}$ & $\mathrm{K}^{-1}$ & Soret coefficient with respect to the substance $\check{l}$ \\
\hline $\mathbf{s}$ & $\mathrm{N} / \mathrm{m}^{2}$ & stress vector \\
\hline $\mathbf{S}$ & $\mathrm{N} / \mathrm{m}^{2}$ & stress tensor \\
\hline $\mathbf{S}(\mathbf{r}) / S_{i^{\prime} j^{\prime}}$ & $\mathrm{N} / \mathrm{m}^{2}$ & stress tensor / element $i^{\prime} j^{\prime}$ \\
\hline$t$ & s & time \\
\hline$\delta t$ & $\mathrm{~s}$ & interval of time on a mesoscopic scale \\
\hline$t_{m}$ & $\mathrm{~s}$ & considered times $m$ \\
\hline$t_{T}$ & s & time constant \\
\hline$T$ & $\mathrm{~K}$ & temperature \\
\hline
\end{tabular}




\begin{tabular}{|c|c|c|}
\hline Latin Symbol & Unit & Description \\
\hline$\hat{T}$ & $\mathrm{~K}$ & $\begin{array}{l}\text { Temperature in a thermostated region after the } \\
\text { thermostating }\end{array}$ \\
\hline$T_{C}$ & $\mathrm{~K}$ & temperature in the cold thermostated region \\
\hline$T_{C H}$ & $\mathrm{~K}$ & $0.5\left(T_{C}+T_{H}\right)$ \\
\hline$T_{H}$ & $\mathrm{~K}$ & temperature in the hot thermostated region \\
\hline$\Delta u$ & $\mathrm{~m} / \mathrm{s}$ & velocity variation across the largest eddies \\
\hline$u_{i j}\left(r_{i j}\right)$ & $\mathrm{J}$ & pair potential between the particles $i$ and $j$ \\
\hline$u_{x, i}$ or $\hat{u}_{x, i}$ & $\mathrm{~m} / \mathrm{s}$ & $\begin{array}{l}x \text {-velocity of the particle } i \text { before or after the } \\
\text { thermostating }\end{array}$ \\
\hline$U$ & $\mathrm{~J}$ & potential energy \\
\hline $\mathbf{u} / u_{i^{\prime}}$ & $\mathrm{m} / \mathrm{s}$ & particle velocity / in $i^{\prime}$ direction \\
\hline $\mathbf{u}_{i} / u_{i, i^{\prime}}$ & $\mathrm{m} / \mathrm{s}$ & velocity of the particle $i$ / in $i^{\prime}$ direction \\
\hline$v_{T C}$ & $\mathrm{~m} / \mathrm{s}$ & uniform thermal creep velocity \\
\hline$v_{V S}$ & $\mathrm{~m} / \mathrm{s}$ & viscous slip velocity \\
\hline$V$ & $\mathrm{~m}^{3}$ & volume \\
\hline$\delta V$ & $\mathrm{~m}^{3}$ & macroscopically small volume element \\
\hline$\Delta V$ & $\mathrm{~m}^{3}$ & macroscopic volume element \\
\hline $\mathbf{v}$ & $\mathrm{m} / \mathrm{s}$ & velocity vector \\
\hline$\delta \dot{w}$ & $\mathrm{~W} / \mathrm{m}^{3}$ & work done by a system \\
\hline$x_{\check{l}}$ & - & mole fraction of the substance $\check{l}$ \\
\hline$x_{\check{i}, 0}$ & - & equilibrium mole fraction of the substance $\iota$ \\
\hline
\end{tabular}

$x_{i j}, y_{i j}, z_{i j} \quad \mathrm{~m} \quad x-, y-, z$ - components of the connecting vector between the particles $i$ and $j$

$y_{C} \quad \mathrm{~m} \quad y$-location of the cold thermostated region

$y_{H} \quad \mathrm{~m} \quad y$-location of the hot thermostated region

$z_{f f 1} \quad \mathrm{~m} \quad z$-coordinate beyond which the external force ff1 increases

$z_{l b ;} z_{u b} \quad \mathrm{~m} \quad$ lower and upper $z$-coordinates between the external force fields ff 2 , ff 3 , ff4 vary

Z $\quad$ - $\quad$ compressibility factor 


\begin{tabular}{|c|c|c|}
\hline \multirow{2}{*}{$\begin{array}{r}\text { Greek Symbol } \\
\qquad \hat{\alpha}\end{array}$} & Unit & Description \\
\hline & $\mathrm{K}^{-1}$ & thermal expansion coefficient \\
\hline$\alpha_{x}$ & - & $\begin{array}{l}\text { scaling factor of the particle } x \text {-velocity in the } \\
\text { thermostating }\end{array}$ \\
\hline$\beta_{x}$ & $\mathrm{~m} / \mathrm{s}$ & shift of the particle $x$-velocity in the thermostating \\
\hline$\gamma$ & $\mathrm{N} / \mathrm{m}$ & surface tension \\
\hline$\gamma_{\check{i}}$ & - & activity coefficient of the substance $\check{l}$ \\
\hline$\varepsilon_{i j}$ & $\mathbf{J}$ & $\begin{array}{l}\text { LJ interaction parameter between the particles } i \text { and } j \text {, } \\
\text { e.g. } \varepsilon_{A r A-A r B}\end{array}$ \\
\hline$\zeta_{A r A}$ or $\zeta_{A r B}$ & $\mathrm{~m}$ & $\begin{array}{l}z \text {-coordinate of the Gibbs dividing surface for the first or } \\
\text { for the second substance }\end{array}$ \\
\hline$\zeta_{V S}$ & $\mathrm{~m}$ & viscous slip coefficient \\
\hline$\zeta_{\check{i},-;} \zeta_{\check{r},+}$ & $\mathrm{m}$ & $\begin{array}{l}z \text {-coordinate of the Gibbs dividing surface in the partial } \\
\text { densities of the substance } \check{l}(z<0 \text { or } z>0)\end{array}$ \\
\hline$\eta$ & - & Lorentz-Berthelot mixing parameter \\
\hline$\tilde{\eta}$ & $\mathrm{kg} /(\mathrm{m} \cdot \mathrm{s})$ & dynamic viscosity \\
\hline $\mathbf{t}_{\check{l}} / \mathfrak{i}_{i^{\prime}, \check{l}}$ & $\mathrm{~N}$ & $\begin{array}{l}\text { interatomic force on a particle of the substance } \check{l} / \text { in } i^{\prime} \text { - } \\
\text { direction }\end{array}$ \\
\hline$l_{z, f f 1}, l_{z, f f 2}, l_{z, f f 3}, l_{z, f f 4}$ & $\mathrm{~N}$ & additional external $z$-force of the type $\mathrm{ff} 1, \mathrm{ff} 2, \mathrm{ff} 3, \mathrm{ff} 4$ \\
\hline $\mathbf{\kappa}_{\check{l}} / \kappa_{i^{\prime}, \check{l}}$ & $\mathrm{~N}$ & $\begin{array}{l}\text { kinetic force on a particle of the substance } \check{l} / \text { in } i^{\prime} \text { - } \\
\text { direction }\end{array}$ \\
\hline$\kappa_{T}$ & $\mathrm{~m}^{2} / \mathrm{N}$ & isothermal compressibility factor \\
\hline$\lambda$ & $\mathrm{m}$ & mean free path of a gas molecule \\
\hline$\lambda_{0}$ & $\mathrm{~m}$ & characteristic dimension of the dissipative eddies \\
\hline$\lambda_{t h}$ & $\mathrm{~W} /(\mathrm{m} \cdot \mathrm{K})$ & thermal conductivity \\
\hline$\mu(o)$ & & $\begin{array}{l}\text { time average of the instantaneous function values } \\
\text { underlying the observable } o\end{array}$ \\
\hline$\mu_{s}(o)$ & & $\begin{array}{l}\text { time average of the instantaneous function values } \\
\text { underlying the observable, } o \text {, over certain segment, } s \text {, of } \\
\text { time }\end{array}$ \\
\hline$\xi$ & - & Lorentz-Berthelot mixing parameter \\
\hline$\Pi$ & $\mathrm{N} / \mathrm{m}^{2}$ & local pressure \\
\hline$\rho$ & $\mathrm{m}^{-3}$ & particle density \\
\hline $\bar{\rho}$ & $\mathrm{kg} / \mathrm{m}^{3}$ & density \\
\hline$\hat{\rho}(\mathbf{q}, \mathbf{p}, t)$ & & statistical distribution function \\
\hline$\rho_{\check{l}}^{\check{L}}$ & $\mathrm{~m}^{-3}$ & partial particle density of the substance $\check{l}$ \\
\hline$\rho_{i}(\check{\mathbf{j}})$ & $\mathrm{m}^{-3}$ & $\begin{array}{l}\text { partial density of the substance } \check{l} \text { in the phase rich in the } \\
\text { substance } \breve{\jmath}, \text { e.g. } \rho_{A r A}(\mathrm{ArB})\end{array}$ \\
\hline
\end{tabular}




\begin{tabular}{|c|c|c|}
\hline $\begin{array}{r}\text { Greek Symbol } \\
\rho_{i j}\left(r_{i j}\right) \\
\sigma(o)\end{array}$ & $\begin{array}{l}\text { Unit } \\
\mathrm{m}^{-6}\end{array}$ & $\begin{array}{l}\text { Description } \\
\text { pair distribution function of the particles } i \text { and } j \\
\text { standard deviation of the instantaneous function values } \\
\text { underlying the observable } o\end{array}$ \\
\hline$\sigma_{i j}$ & $\mathrm{~m}$ & $\begin{array}{l}\text { LJ interaction parameter between the particles } i \text { and } j \text {, } \\
\text { e.g. } \sigma_{A r A-A r B}\end{array}$ \\
\hline$\tau$ & s & arbitrarily long time interval \\
\hline$\Phi$ & $\mathrm{s}^{3} / \mathrm{m}^{6}$ & particle distribution function \\
\hline$\varphi_{\check{l}}$ & - & fugacity coefficient of the substance $\check{l}$ in a mixture \\
\hline$\varphi_{\check{L}, 0}$ & - & fugacity coefficient of the pure substance $\check{l}$ \\
\hline $\boldsymbol{\varphi}_{\grave{i}} / \varphi_{i^{\prime}, \check{l}}$ & $\mathrm{~N}$ & force per particle of the substance $\check{l} /$ in $i^{\prime}$-direction \\
\hline$\omega$ & $\mathrm{m}$ & interfacial width \\
\hline$\omega_{\check{\imath}}$ & $\mathrm{m}$ & $\begin{array}{l}\text { interfacial width related to the densities of the } \\
\text { component } \check{l}\end{array}$ \\
\hline
\end{tabular}




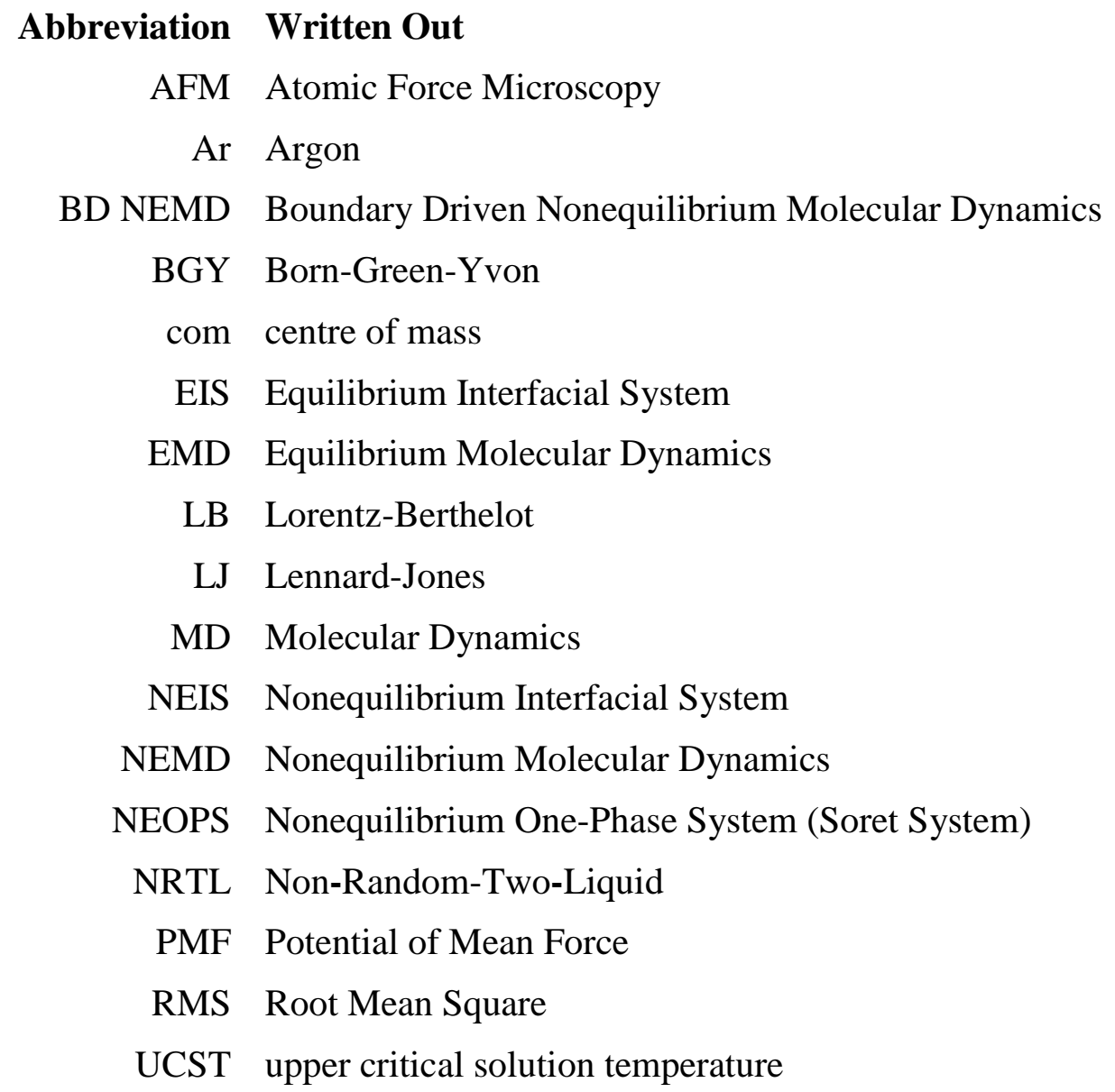

